\title{
Implementing pharmacogenetics to personalize antiplatelet therapy after myocardial infarction
}

Citation for published version (APA):

Claassens, D. M. F. (2021). Implementing pharmacogenetics to personalize antiplatelet therapy after myocardial infarction. [Doctoral Thesis, Maastricht University]. Maastricht University. https://doi.org/10.26481/dis.20211203dc

Document status and date:

Published: 01/01/2021

DOI:

10.26481/dis.20211203dc

Document Version:

Publisher's PDF, also known as Version of record

\section{Please check the document version of this publication:}

- A submitted manuscript is the version of the article upon submission and before peer-review. There can be important differences between the submitted version and the official published version of record.

People interested in the research are advised to contact the author for the final version of the publication, or visit the DOI to the publisher's website.

- The final author version and the galley proof are versions of the publication after peer review.

- The final published version features the final layout of the paper including the volume, issue and page numbers.

Link to publication

\footnotetext{
General rights rights.

- You may freely distribute the URL identifying the publication in the public portal. please follow below link for the End User Agreement:

www.umlib.nl/taverne-license

Take down policy

If you believe that this document breaches copyright please contact us at:

repository@maastrichtuniversity.nl

providing details and we will investigate your claim.
}

Copyright and moral rights for the publications made accessible in the public portal are retained by the authors and/or other copyright owners and it is a condition of accessing publications that users recognise and abide by the legal requirements associated with these

- Users may download and print one copy of any publication from the public portal for the purpose of private study or research.

- You may not further distribute the material or use it for any profit-making activity or commercial gain

If the publication is distributed under the terms of Article $25 \mathrm{fa}$ of the Dutch Copyright Act, indicated by the "Taverne" license above, 
Implementing pharmacogenetics to personalize antiplatelet therapy after myocardial infarction

Daniel Marcus Franciscus Claassens 


\title{
Implementing pharmacogenetics to personalize
}

\section{antiplatelet therapy after myocardial infarction}

\author{
Proefschrift
}

ter verkrijging van de graad van doctor aan de Universiteit Maastricht, op gezag van de Rector Magnificus, Prof. dr. Rianne M. Letschert

volgens het besluit van het College van Decanen,

in het openbaar te verdedigen

Implementing pharmacogenetics to personalize antiplatelet therapy after myocardial infarction

op vrijdag 3 december 2021 om 14.00 uur

$\begin{array}{ll}\text { ISBN } & 978-94-6423-437-4 \\ \text { Author } & \text { D.M.F. Claassens } \\ \text { Cover } & \text { B. Jaspers, ProefschriftOntwerp.nl } \\ \text { Lay-out } & \text { B. Jaspers \& ProefschriftMaken } \\ \text { Print } & \text { ProefschriftMaken }\end{array}$

door

CC D.M.F. Claassens, 2021, Utrecht, The Netherlands

No parts of this thesis may be reproduced or transmitted in any form or by any means without permission of the author. The copyright of the articles that have been published or have been accepted for publication has been transferred to the respective journals.

The publication of this thesis was supported by the Wetenschapsfonds Medisch Specialisten and the Team Interventiecardiologie of the Isala Zwolle, the R\&I Heart Center and the Raad van Bestuur of the St. Antoniusziekenhuis Nieuwegein, the Universiteit Maastricht, ChipSoft, Boston Scientific, Medtronic and R.B.A. Claassens \& M.J.S. Claassensvan Daalen.

Daniel Marcus Franciscus Claassens

Geboren op 14 maart 1993 te Rotterdam 


\section{Promotores}

Prof. dr. J.M. ten Berg

Prof. dr. A.W.J. van 't Hof

\section{Copromotores}

Dr. V.H.M. Deneer (UMC Utrecht, Utrecht)

Dr. R.S. Hermanides (Isala, Zwolle)

\section{Beoordelingscommissie}

Prof. dr. T.M. Hackeng (voorzitter)

Prof. dr. H. ten Cate

Prof. dr. R.J.M. van Geuns (Radboud Universiteit, Nijmegen)

Prof. dr. J.P.S. Henriques (Amsterdam UMC - AMC, Amsterdam)

Prof. dr. K. Vernooy

Financial support by the Dutch Heart Foundation for the publication of

this thesis is gratefully acknowledged. 
Part I

Introduction

General introduction and thesis outline

Chapter 1 Genotype-guided treatment of oral P2Y12

inhibitors: Where do we stand?

Pharmacogenomics 2020; 21:83-86

Chapter 2 De-escalation of antiplatelet treatment in patients with myocardial infarction who

underwent percutaneous coronary intervention:

a review of the current literature

Journal of Clinical Medicine 2020; 9:2983

Part II

Outcomes of using CYP2C19 genetic testing in

clinical practice

Chapter 3 Feasibility and implementation of CYP2C19

genotyping in patients using antiplatelet therapy

Pharmacogenomics 2018; 19:621-628

Chapter 4 A genotype-guided strategy for oral P2Y12

inhibitors in primary $\mathrm{PC}$

New England Journal of Medicine 2019; 381:1621-1631

Chapter 5 Clopidogrel versus ticagrelor or prasugrel after primary percutaneous coronary intervention according to CYP2C19 genotype. A POPular genetics subanalysis

Circulation: Cardiovascular Interventions 2021; 14:e009434 function alleles versus ticagrelor in elderly

patients with acute coronary syndrome: A pre-

specified sub analysis from the POPular Genetics and POPular Age trials

International Journal of Cardiology 2021; 334:10-17

Chapter 7 Cost-effectiveness of a CYP2C19 genotypeguided strategy in acute myocardial infarction patients: Results from the POPular Genetics tria

American Journal of Cardiovascular Drugs 2021;

DOI: 10.1007/s40256-021-00496-4

Part III

Switching P2Y12 inhibitors in the early phase after STEMI

Chapter 8

Reloading when switching from ticagrelor or

STEMI

JACC: Cardiovascular Interventions 2020; 13:663-665

General discussion

Nederlandse samenvatting

Impact section

Curriculum vitae

Dankwoord 


\section{Part I: Introduction}

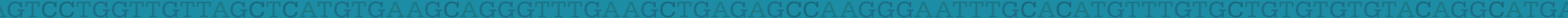

AAGAGTTAAAGAGCAGTGTTTTTCCCATTATCTATTCCAGAACATGTCACCAGAGAATACTTGACAAGTGGACATGGTGGAAATC
CATTCTGGCCAGTAATGACATTCTACAGTTTTATTGCTTAGGATATCTTAGTGTAGTTCTCATCAGCTTTTATTTCTCTGTAA

TTAACTAGATTTTAATTATTAGTAATAACTGTAATTTTTATTCCTTAAGTATAATTTGGCTCTGTTTCATTTTGCTTATCTCTTTCCAAT
TTAACAAGAGGAGAAGGCTTCAATGGATCCTTTTGTGGTCCTTGTGCTCTGTCTCTCATGTTTGCTTCTCCTTCAATCTGGAGACAC 


\section{General introduction and thesis outline}

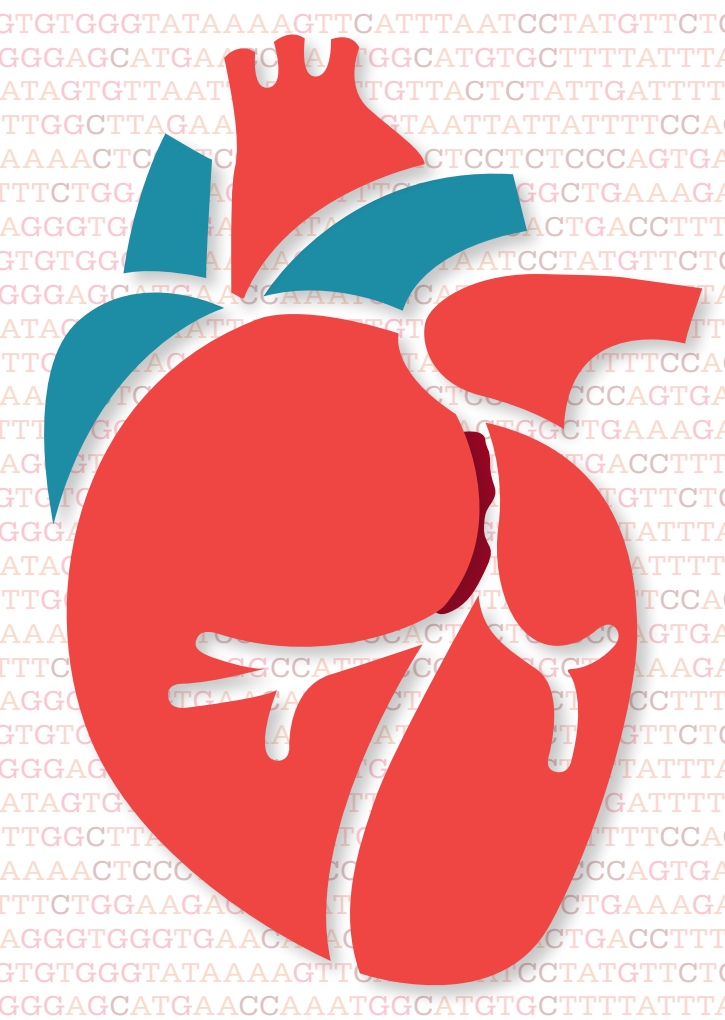


Antiplatelet agents are prescribed to reduce blood platelet aggregation. This plays an important role in occlusion of coronary vessels. It is recommended that patients with an acute coronary syndrome (ACS) and patients undergoing percutaneous coronary intervention (PCI) are treated with dual antiplatelet therapy (DAPT) consisting of aspirin and a $\mathrm{P} 2 \mathrm{Y}_{12}$ inhibitor $[1,2]$. DAPT has proven to reduce atherothrombotic event rates such as stent thrombosis and recurrent myocardial infarction as compared to aspirin monotherapy in the first months and year(s) after an ACS or PCI [3]. The antithrombotic effect of aspirin is mediated by the irreversible inhibition of platelet cyclooxygenase-1, which leads to inhibition of Thromboxane $\mathrm{A}_{2}$ synthesis. Thromboxane $\mathrm{A}_{2}$ is part of the platelet activation pathway that leads to thrombus formation. Several different ora $\mathrm{P}_{2} \mathrm{Y}_{12}$ inhibitors are prescribed, but they all work by binding to the $\mathrm{P} 2 \mathrm{Y}_{12}$ receptor. This prevents binding of adenosine diphosphate to the $\mathrm{P} 2 \mathrm{Y}_{12}$ receptor, which is an important mediator in platelet aggregation.

\section{P2Y 12 inhibitors}

\section{Clopidogrel}

Clopidogrel is a prodrug. It is converted into its active metabolite by cytochrome P450 isoenzymes. After metabolizing, it irreversibly binds to the $\mathrm{P} 2 \mathrm{Y}_{12}$ receptor. The most important enzyme in the two-step conversion of clopidogrel is the CYP2C19 enzyme. The CYP2C19 gene encodes this enzyme. Many different polymorphisms of the CYP2C19 gene have been identified, but most are rare [4]. The CYP $C 19^{*} 1$ allele is considered the normal function allele, while the $* 17$ allele is the most common polymorphism. The $* 17$ allele is considered to be a gain-of-function allele by some, because it would lead to increased platelet inhibition as compared to the $* 1$ allele. However, data on this effect is conflicting and an effect on clinical outcomes has not been well-established [4-5]. The *2 is the most common allele leading to a reduced function (loss-of-function), while the *3 loss-of-function allele is quite common in some populations (mostly Asian) and rare in others (American) [4]. Therefore, these two polymorphisms are the most clinically relevant.

Approximately one-third of patients treated with clopidogrel have high ontreatment platelet reactivity when measured with platelet function tests [6]. This is associated with an increased risk of experiencing atherothrombotic events [6]. Carrying CYP2C19 loss-of-function alleles is one of the most important risk factors for high on-treatment platelet reactivity [7-9]. This has prompted the United States Food and Drug Administration (FDA) to issue a boxed warning that in people carrying two loss-of-function alleles an alternative for clopidogrel should be considered [10]. Testing for the presence of CYP2C19 loss-of-function alleles could help identify those patients who should not be treated with clopidogrel, but with one of the other $\mathrm{P}_{2} \mathrm{Y}_{12}$ inhibitors instead.

\section{Prasugrel and ticagrelor}

Prasugrel is a prodrug, which irreversibly binds to the $\mathrm{P} 2 \mathrm{Y}_{12}$ receptor, like clopidogrel, but its effect is not affected by CYP2C19 polymorphisms [11]

Ticagrelor is a direct-acting $\mathrm{P}_{2} \mathrm{Y}_{12}$ inhibitor and is therefore not dependen on CYP2C19 metabolism [12]. Both drugs have demonstrated to be more potent platelet inhibitors and have a more consistent pharmacodynamic effect than clopidogrel [13-14].

Initially, after publication of the prospective Clopidogrel in Unstable angina to prevent Recurrent Events (CURE) trial, clopidogrel became the recommended $\mathrm{P} 2 \mathrm{Y}_{12}$ inhibitor in addition to aspirin [3]. The introduction of prasugrel and ticagrelor a few years later led to even lower levels of platelet aggregation, thus preventing even more atherothrombotic events [13-14]. Due to the reduction in atherothrombotic events, both American and European guidelines recommend the use of ticagrelor and prasugre instead of clopidogrel in patients with an ACS, unless there is a contraindication for these drugs. [1,2,15]. However, more intensified platele inhibition does not only decrease the number of atherothrombotic events, but inevitably also leads to more bleeding events [13-14]. Bleeding is associated with a reduction in quality of life, extra healthcare costs and mortality [16]. Therefore, it is important to not only prevent atherothrombotic events, but bleeding events as well.

\section{De-escalation of antiplatelet therapy}

The highest risk of recurrent atherothrombotic events is seen early after an ACS, while the bleeding risk remains consistent during the period a $\mathrm{P} 2 \mathrm{Y}_{12}$ inhibitor is used [13-14]. This has prompted a theory that switching from the stronger $\mathrm{P}_{2} \mathrm{Y}_{12}$ inhibitors ticagrelor or prasugrel to clopidogrel in the period after an ACS could reduce the overall bleeding risk, without increasing the thrombotic risk. This concept is called de-escalation. Three different strategies have been investigated to test this theory. A more detailed description is provided later in this thesis, but the three different strategies are unguided de-escalation (switching without using any test), platelet function testing-guided de-escalation and CYP2C19 genotypeguided de-escalation [16]. 


\section{Switching between different $\mathrm{P}_{2} \mathrm{Y}_{12}$ inhibitors}

Switching between $\mathrm{P}_{2} \mathrm{Y}_{12}$ inhibitors is common in clinical practice, either as part of a conscious strategy, due to concomitant therapies, side effects or for socio-economic reasons $[16,17]$. Since the different $\mathrm{P}_{2} \mathrm{Y}_{12}$ inhibitors have different pharmacological properties, concerns have been raised for drug-drug interactions (DDIs). A DDI is defined as a modification of the effect of a drug when administered with another drug. In the case of $\mathrm{P} 2 \mathrm{Y}_{12}$ inhibitors, a DDI could lead to inadequate platelet inhibition and an increased risk of atherothrombotic complications when switching. Based on pharmacodynamic and pharmacokinetic data from the different drugs, an expert consensus statement has been published to provide handles for clinicians on how to switch between $\mathrm{P}_{2} \mathrm{Y}_{12}$ inhibitors [17]. When deescalating from ticagrelor or prasugrel to clopidogrel, it is recommended to administer a loading dose of $600 \mathrm{mg}, 24$ hours after the last dose of prasugrel or ticagrelor. When de-escalation is performed due to bleeding, instead of a loading dose, a maintenance dose of $75 \mathrm{mg}$ can be considered 24 hours after the last dose of ticagrelor or prasugrel. However, a paucity of studies investigating the effect of different strategies on clinical outcomes still exist.

\section{Outline of the thesis}

The focus of this thesis will be on CYP2C19 genotype-guided deescalation and results of the CYP2C19 Genotype-Guided Antiplatelet Therapy in ST-Segment Elevation Myocardial Infarction Patients - Patient Outcome after Primary PCI (POPular Genetics) trial. Chapter 1 starts with an overview of the current status of CYP2C19 genotyping. Chapter 2 focuses on the feasibility of using CYP2C19 genetics testing in clinical practice. It describes the time it takes until genetic results are available and reports results on both a test performed in a laboratory and a point-of-care system. Chapter 3 provides an overview of the different strategies that can be used to de-escalate antiplatelet therapy. It presents data from trials that have studied the different strategies, provides pros and cons of the different strategies and provides handles on when and how to de-escalate. In Chapter 4 the clinical outcomes of the POPular Genetics trial are presented. It investigated the effect of a CYP2C19 genotype-guided strategy compared to standard treatment with ticagrelor or prasugrel on atherothrombotic and bleeding events. Chapter $\mathbf{5}$ is a sub-analysis of the POPular Genetics trial, which, instead of comparing the two different strategies, compared clopidogrel in noncarriers of CYP2C19 loss-offunction alleles directly with ticagrelor and prasugrel. Chapter 6 is a subanalysis from both the POPular Genetics and POPular Age trials. It also compares clopidogrel in noncarriers of CYP2C19 loss-of-function alleles with ticagrelor and prasugrel in a subgroup of elderly patients with ACS In Chapter 7, the cost-effectiveness of a CYP2C19 genotype-guided strategy compared to standard treatment with ticagrelor and prasugrel is investigated. The final chapter, Chapter 8, describes outcomes of patients de-escalating to clopidogrel with and without administering a new loading dose. 


\section{References}

1. Ibanez B, James S, Agewall S, et al. 2017 ESC guidelines for the management of acute myocardial infarction in patients presenting with STsegment elevation. The Task Force for the management of acute myocardial infarction in patients presenting with ST-segment elevation of the European Society of Cardiology (ESC). Eur Heart J 2018; 39:119-177

2. Collet JP, Thiele H, Barbato E, et al. 2020 ESC Guidelines for the management of acute coronary syndromes in patients presenting without persistent ST-segment elevation. Eur Heart J 2021; 42:1289-1367

3. Yusuf S, Zhao F, Mehta SR, et al. Effects of Clopidogrel in Addition to Aspirin in Patients with Acute Coronary Syndromes without ST-Segment Elevation N Engl J Med 2001; 345:494-502

4. Moon JY, Franchi F, Rollini F, et al. Role of genetic testing in patients undergoing percutaneous coronary intervention. Expert Rev Clin Pharmacol 2018; 11:151-164

5. Lewis JP, Stephens SH, Horenstein RB, et al. The CYP2C19*17 variant is not independently associated with clopidogrel response. J Thromb Haemost $2013 ; 11: 1640-1646$

6. Breet NJ, Van Werkum JW, Bouman HJ, et al. Comparison of platelet function tests in predicting clinical outcome in patients undergoing coronary stent implantation. JAMA 2010; 303:754-762

7. Shuldiner AR, O'Connell JR, Bliden KP, et al. Association of cytochrome P450 2C19 genotype with the antiplatelet effect and clinical efficacy of clopidogrel therapy. JAMA 2009; 302:849-857

8. Simon T, Verstuyft C, Mary-Krause M, et al. Genetic determinants of response to clopidogrel and cardiovascular events. N Engl J Med 2009; 360:363-375

9. Mega JL, Simon T, Collet JP, et al. Reduced-function CYP2C19 genotype and risk of adverse clinical outcomes among patients treated with clopidogrel predominantly for PCI: a meta-analysis. JAMA 2010 304:1821-1830

10. Holmes DR, Dehmer GJ, Kaul S, et al. ACCF/AHA clopidogrel clinical alert: approaches to the FDA "boxed warning": a report of the American College of Cardiology foundation task force on clinical export consensus documents and the American Heart Association endorsed by the Society of Cardiovascular Angiography and Interventions and the Society of Thoracic Surgeons. J Am Coll Cardiol 2010; 56:321-341

11. Mega JL, Close SL, Wiviott SD, et al. Genetic variants in ABCB1 and CYP2C19 and cardiovascular outcomes after treatment with clopidogrel and prasugrel in the TRITON-TIMI 38 trial: a pharmacogenetic analysis. Lancet 2010; 376:1312-1319

12. Wallentin L, James S, Storey RF, et al. Effect of CYP2C19 and ABCB1 single nucleotide polymorphisms on outcomes of treatment with ticagrelor versus clopidogrel for acute coronary syndromes: a genetic substudy of the PLATO trial. Lancet 2010; 376:1320-1328
13. Wallentin L, Becker RC, Budaj A, et al. Ticagrelor versus clopidogrel in patients with acute coronary syndromes. N Engl J Med 2009; 361:10451057

14. Wiviott SD, Braunwald E, McCabe $\mathrm{CH}$, et al. Prasugrel versus clopidogrel in patients with acute coronary syndromes. N Engl J Med 2007; 357:20012015

15. Levine GN, Bates ER, Bittl JA, et al. 2016 ACC/AHA guideline focused update on duration of dual antiplatelet therapy in patients with coronary artery disease: a report of the American College of Cardiology/American Heart Association Task Force on Clinical Practice Guidelines. J Am Coll Cardiol 2016; 68:1082-1115

16. Claassens DMF, Sibbing D. De-Escalation of Antiplatelet Treatment in Patients with Myocardial Infarction Who Underwent Percutaneous Coronary Intervention: A Review of the Current Literature. J Clin Med 2020; 9:2983

17. Angiolillo DJ, Rollini F, Storey RF, et al. International Expert Consensus on Switching Platelet P2Y12 Receptor Inhibiting Therapies. Circulation 2017; 136:1955-1975 
Genotype-guided treatment of oral $\mathrm{P}_{2} \mathrm{Y}_{12}$ inhibitors:

Where do we stand?

Daniel M.F. Claassens, Jurriën M. ten Berg

Pharmacogenomics 2020; 21:83-86
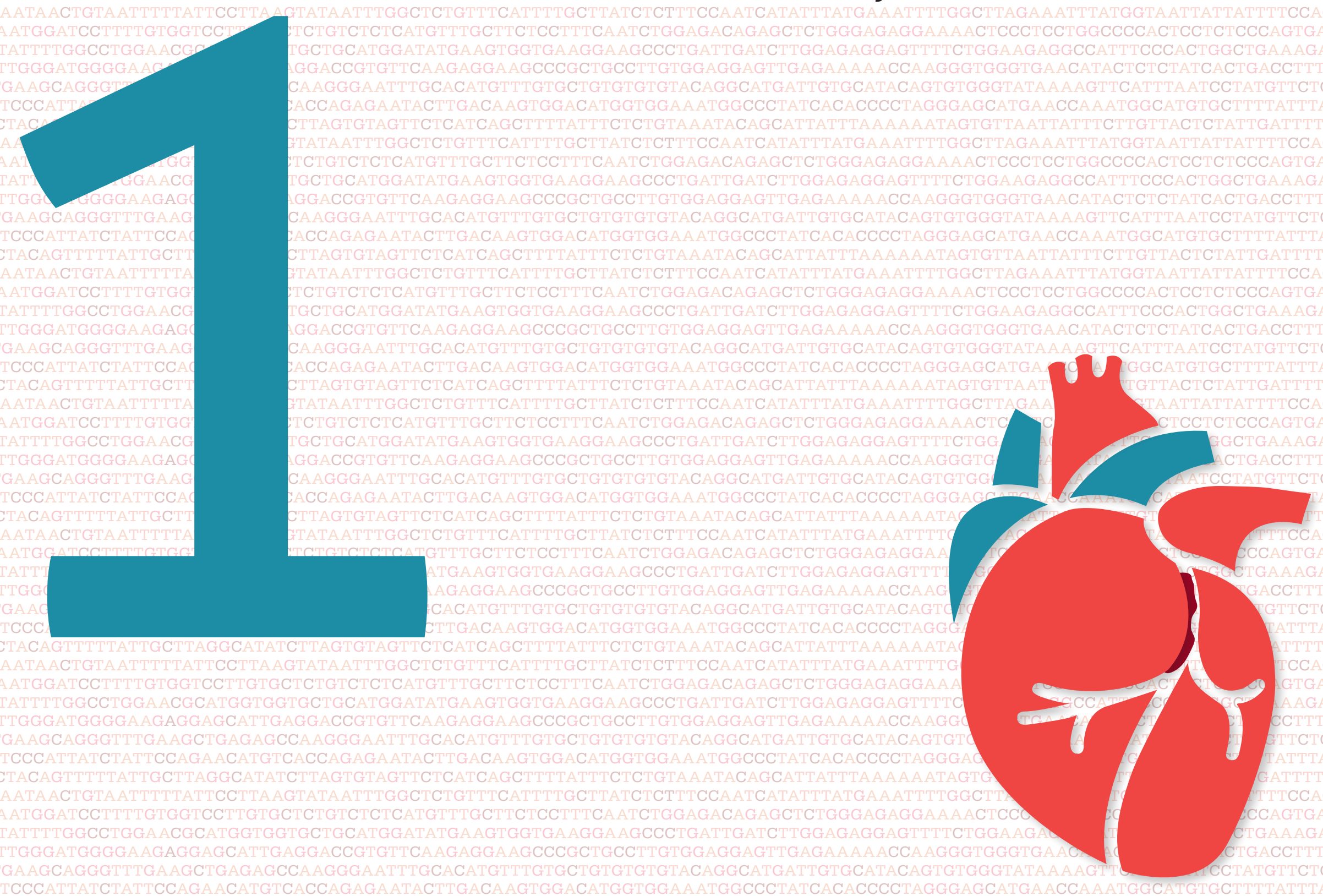


\section{Introduction}

Dual antiplatelet therapy, consisting of aspirin and a $\mathrm{P}_{2} \mathrm{Y}_{12}$ inhibitor remains the cornerstone of treatment in preventing recurrent thrombotic events such as stent thrombosis or spontaneous myocardial infarction (MI) after percutaneous coronary intervention (PCI) or acute coronary syndrome (ACS). In patients with ACS, the guidelines favour the stronger $\mathrm{P} 2 \mathrm{Y}_{12}$ inhibitors ticagrelor or prasugrel over weaker clopidogrel $[1,2]$. This recommendation is based on the Platelet Inhibition and Patient Outcomes (PLATO) trial which demonstrated ticagrelor was superior in reducing MI and death as compared with clopidogrel and on the Trial to Assess Improvement in Therapeutic Outcomes by Optimizing Platelet Inhibition with Prasugrel-Thrombolysis in Myocardial Infarction 38, which demonstrated prasugrel was superior in reducing MI as compared with clopidogrel $[3,4]$. However, the improved efficacy of these stronger platelet inhibitors is counterbalanced by a higher bleeding risk.

Clopidogrel is a prodrug, which is transformed into its active metabolite by hepatic cytochrome P450 enzymes. The active metabolite irreversibly inhibits the $\mathrm{P}_{2} \mathrm{Y}_{12}$ receptor on platelets, resulting in inhibition of platelet aggregation [5]. However, clopidogrel-treated patients demonstrate a substantial individual response variability [6]. The most important gene to cause reduced clopidogrel effectiveness is the CYP2C19 gene [7-9]. Carriers of CYP2C19 loss-of-function (LoF) alleles are at an increased risk of thrombotic events [10-12]. This has prompted the US FDA and the European Medicines Agency to add a boxed warning to the label, describing that clopidogrel might not be effective in poor metabolizers [13].

More than 30 gene alleles have been identified [14]. The CYP2C19*1 is the most prevalent $(60-70 \%$ of the population) and is regarded as the allele causing normal activity. CYP2C19*2 -*8 are LoF alleles. Of these, the CYP2C19*2 (15-35\%) and, depending on the population, *3 $(0.02-9 \%)$ alleles are, by far, the most common LoF alleles [15]. The CYP $2 C 19 * 17$ is regarded as a gain-of-function allele by many. Based on these assumptions, patients are divided into four different phenotype categories (Table 1) [16] However, Lewis et al. demonstrated that CYP2C19*17 is not independently associated with clopidogrel response [17]. Furthermore, data on the influence of the CYP2C19*17 allele on clinical outcomes are inconsistent and its clinical relevance is therefore questionable [15].

Despite the better efficacy of the stronger $\mathrm{P}_{2} \mathrm{Y}_{12}$ inhibitors, clopidogrel remains the most utilized platelet inhibitor of the three [15]. The reduced risk of bleeding and reduced costs, especially in non-western countries, probably plays an important part in this. In addition to the introduction of the stronger $\mathrm{P}_{2} \mathrm{Y}_{12}$ inhibitors, the introduction of newer generation stents have caused a dramatic decrease in thrombotic events, which has shifted attention to the prevention of bleeding complications [18]. This has spurred on research that tries to combine the improved efficacy of the stronger $\mathrm{P} 2 \mathrm{Y}_{12}$ inhibitors in patients that are unable to metabolize clopidogrel with the reduced risk of bleeding in patients that react properly to clopidogrel [19-21]. This can be achieved in two ways, either by using proof from platelet function testing or from genetic testing. Furthermore, it is possible to either choose a strategy in which these tests are used to escalate a strategy from clopidogrel to the stronger $\mathrm{P} 2 \mathrm{Y}_{12}$ inhibitors or a de-escalation strategy from the stronger $\mathrm{P}_{2} \mathrm{Y}_{12}$ inhibitors to clopidogrel [21].

\section{Table 1. CYP2C19 phenotypes based on genotypes}

\begin{tabular}{|lll|}
\hline Phenotype & Genotypes & Examples of diplotypes \\
\hline $\begin{array}{l}\text { (Ultra)rapid } \\
\text { metabolizer } ¥\end{array}$ & $\begin{array}{l}\text { Carriers of one or two } \\
\text { gain-of-function alleles }\end{array}$ & $* 1 / * 17 . * 17 / * 17$ \\
\hline $\begin{array}{l}\text { Extensive } \\
\text { metabolizer }\end{array}$ & $\begin{array}{l}\text { Non-carrier of loss-of- } \\
\text { function alleles }\end{array}$ & $* 1 / * 1$ \\
\hline $\begin{array}{l}\text { Intermediate } \\
\text { metabolizer }\end{array}$ & $\begin{array}{l}\text { Carriers of one loss-of- } \\
\text { function allele }\end{array}$ & $* 1 / * 2, * 1 / * 3$ \\
\hline Poor metabolizer & $\begin{array}{l}\text { Carriers of two loss-of- } \\
\text { function alleles }\end{array}$ & $\begin{array}{l}* 2 / * 2, * 2 / * 3, * 3 / * 3 \text { or } \\
\text { combinations of two } \\
\text { other LoF alleles }(* 2- \\
\end{array}$ \\
& & $* 8)$ \\
\hline
\end{tabular}

Table 1. $¥$ Data on the existence of a gain-of-function allele are conflicting. LoF: Loss-of-function 


\section{Feasibility}

There have been many observational studies and randomized trials investigating the use of platelet function testing. However, there has never been a randomized trial which demonstrated a benefit in using platelet function testing over standard treatment Still, the latest European Society of Cardiology guidelines on myocardial revascularization give a class IIB recommendation to use platelet function testing to guide de-escalation of P2 $\mathrm{Y}_{12}$ inhibitor therapy in ACS patients [22]. However, there are some distinct advantages of genotyping over platelet function testing when it comes to feasibility. First, a point-of-care system like the Spartan Cube (Spartan Bioscience, Ontario, Canada) only requires a buccal swab, which is patient friendly and easy to use and returns a result in one hour [19]. Second, genetic testing can be performed at any time, either prior to elective PCI or in the Cath lab in ST-segment elevation MI patients since it does not require stable platelet reactivity measurements to provide a reliable result [23]. Third, compared with the strategy used in for instance the Testing Responsiveness to Platelet Inhibition on Chronic Antiplatelet Treatment for Acute Coronary Syndromes trial, there is no need to return to the hospital for measuring platelet reactivity. At last, only one switch of P2 $\mathrm{Y}_{12}$ inhibitors is necessary instead of two when patients are unresponsive to clopidogrel and have to be switched back to the potent platelet inhibitors [21].

\section{Escalation using CYP2C19 genotype-guided treatment}

Though the concept of CYP2C19 genotype-guided treatment has been around for some time, larger randomized trials evaluating the concept have been missing until recently. Results of the Reassessment of Anti-Platelet Therapy Using an Individualized Strategy Based on Genetic Evaluation trial, which included 187 patients, were already published in 2012. It demonstrated that one week after initiation of antiplatelet therapy there were significantly less patients with high on-treatment platelet reactivity in the genotype-guided group than in the standard treatment group, in which all patients received clopidogrel [24]. Since then, multiple smaller and mostly single centre trials have been performed [25]. A meta-analysis from Kheiri et al. showed that there was no significant difference in major adverse cardiac events and bleeding events between genotype-guided treatment and standard treatment [25]. They, however, found a significantly lower rate of $\mathrm{MI}$ in the genotype-guided group (Hazard ratio $[\mathrm{HR}]=0.44 ; 95 \%$ CI $0.28-0.70)$. However, this meta-analysis only included 2371 patients and there was a lot of heterogeneity in study population and in the definitions used in the different trials. The trials in the meta-analysis investigated escalation of antiplatelet therapy from clopidogrel to ticagrelor or prasugrel, though these platelet inhibitors were also prescribed in the standard treatment arm in a significant portion of the patients. Currently, there are two larger ongoing randomized trials also investigating escalation of antiplatelet therapy after PCI. In the Tailored Antiplatelet Therapy Following PCI (NCT 01742117), 5300 patients undergoing PCI for stable coronary artery disease and ACS are randomized to standard treatment with clopidogrel or to genetic testing of the CYP2C19*2, *3 and *17 alleles. Carriers of the LoF alleles are treated with ticagrelor, while noncarriers receive clopidogrel. Results of this trial are expected in the first half of 2019. A second trial, conducted in Saudi Arabia (NCT 01823185), is expected to include between 1500 and 2000 patients with STEMI. Patients in the standard treatment arm receive clopidogrel, while in the genotype-guided arm the $C Y P 2 C 19 * 2$ allele is tested. Carriers receive ticagrelor or prasugrel while noncarriers receive clopidogrel. Based on the meta-analysis, there might be an advantage in using genotyping to escalate treatment, but we have to wait for the results of these larger trials before we can draw more definitive conclusions.

\section{De-escalation using CYP2C19 genotype-guided treatment}

Though clopidogrel is still the most widely used $\mathrm{P} 2 \mathrm{Y}_{12}$ inhibitor, the use of the potent $\mathrm{P} 2 \mathrm{Y}_{12}$ inhibitors is preferred in ACS patients [1,2]. Based on this assumption the recently published CYP2C19 Genotype-Guided Antiplatelet Therapy in ST-Segment Elevation Myocardial Infarction Patients - Patient Outcome after Primary PCI (POPular Genetics) trial was conducted. Patients in the standard treatment arm received ticagrelor or prasugrel, while in the genotype-guided arm the CYP2C19*2 and *3 alleles were assessed. Carriers were also treated with ticagrelor or prasugrel, while noncarriers received clopidogrel [19]. The trial included 2488 STEMI patients undergoing primary PCI with more than $90 \%$ of the patients in the standard treatment arm receiving ticagrelor or prasugrel and more than $60 \%$ of the patients in the genotype-guided arm receiving clopidogrel. It concluded that a genotype-guided strategy was noninferior for net clinical benefit consisting of all-cause death, recurrent MI, definite ST, stroke and PLATO major bleeding ( $\mathrm{HR}=0.87 ; 95 \% \mathrm{CI}$ : $0.62-1.21, \mathrm{P}_{\text {non-inferiority }}$ $<0.001$ ), while it was superior in reducing combined PLATO major and minor bleeding ( $\mathrm{HR}=0.78 ; 95 \% \mathrm{CI}$ : $0.61-0.98 ; \mathrm{p}=0.04$ ). This reduction was mainly driven by a reduction in minor bleedings $(\mathrm{HR}=0.72 ; 95 \% \mathrm{CI}$ $0.55-0.94)$, with no difference in major bleeding ( $\mathrm{HR}=0.97 ; 95 \% \mathrm{CI}$ : $0.58-1.63)$. 


\section{Conclusion}

Currently, the guidelines still recommend a one-size-fits-all approach using the more potent platelet inhibitors ticagrelor and prasugrel. However, the POPular Genetics trial demonstrated that a personalized approach using genetic testing to de-escalate to clopidogrel benefits patient outcomes and is feasible in clinical practice in a high-risk STEMI population. In the near future, other trials will provide an answer to the question whether a strategy using genotyping to escalate antiplatelet therapy will also benefit patients.

\section{References}

1. Ibanez B, James S, Agewall S, et al. 2017 ESC guidelines for the management of acute myocardial infarction in patients presenting with STsegment elevation: The task force for the management of acute myocardial infarction in patients presenting with ST-segment elevation of the European Society of Cardiology. Eur Heart J 2018; 39:119-177

2. Levine GN, Bates ER, Bittl JA, et al. 2016 ACC/AHA guideline focused update on duration of dual antiplatelet therapy in patients with coronary artery disease: a report of the American College of Cardiology/American Heart Association Task Force on Clinical Practice Guidelines. J Am Coll Cardiol 2016; 68:1082-1115

3. Wallentin L, Becker RC, Budaj A, et al. Ticagrelor versus clopidogrel in patients with acute coronary syndromes. N Engl J Med 2009; 361:1045-1057

4. Wiviott SD, Braunwald E, McCabe CH, et al. Prasugrel versus clopidogrel in patients with acute coronary syndromes. N Engl J Med 2007; 357:20012015

5. Van Werkum JW, Heestermans AA, Deneer VHM, et al. Clopidogrel resistance: fact and fiction. Future Cardiol 2006; 2:215-228

6. Breet NJ, Van Werkum JW, Bouman HJ, et al. Comparison of platelet function tests in predicting clinical outcome in patients undergoing coronary stent implantation. JAMA 2010; 303:754-762

7. Shuldiner AR, O'Connell JR, Bliden KP, et al. Association of cytochrome P450 2C19 genotype with the antiplatelet effect and clinical efficacy of clopidogrel therapy. JAMA 2009; 302:849-857

8. Simon T, Verstuyft C, Mary-Krause M, et al. Genetic determinants of response to clopidogrel and cardiovascular events. N Engl J Med 2009; 360:363-375

9. Mega JL, Simon T, Collet JP, et al. Reduced-function CYP2C19 genotype and risk of adverse clinical outcomes among patients treated with clopidogrel predominantly for PCI: a meta-analysis. JAMA 2010; 304:1821-1830

10. Angiolilo DJ, Fernando-Ortiz A, Bernardo E, et al. Variability in individual responsiveness to clopidogrel: clinical implications, management and future perspectives. J Am Coll Cardiol 2007; 49:1505-1516

11. Collet JP, Hulot JS, Pena A, et al. Cytochrome P450 2C19 polymorphism in young patients treated with clopidogrel after myocardial infarction: a cohort study. Lancet 2009; 373:309-317

12. Harmsze AM, Van Werkum JW, Ten Berg JM, et al. CYP2C19*2 and CYP2C19*3 alleles are associated with stent thrombosis: a case-control study. Eur Heart J 2010; 31:3046-3053

13. Holmes DR, Dehmer GJ, Kaul S, et al. ACCF/AHA clopidogrel clinical alert: approaches to the FDA "boxed warning": a report of the American College of Cardiology foundation task force on clinical export consensus documents and the American Heart Association endorsed by the Society of Cardiovascular Angiography and Interventions and the Society of Thoracic Surgeons. J Am Coll Cardiol 2010; 56:321-341 
14. Scott SA, Sangkuhl K, Shuldiner AR, et al. PharmGKB summary: very important pharmacogene information for cytochrome P450, family 2, subfamily C, polypeptide 19 . Pharmacogenet Genomics 2012; 22:159-165

15. Moon JY, Franchi F, Rollini F, et al. Role of genetic testing in patients undergoing percutaneous coronary intervention. Expert Rev Clin Pharmacol $2018 ; 11: 151-164$

16. Scott SA, Sangkuhl K, Stein CM, et al. Clinical pharmacogenetics implementation consortium guidelines for CYP2C19 genotype and clopidogrel therapy: 2013 update. Clin Pharmacol Ther 2013; 94:317-323

17. Lewis JP, Stephens SH, Horenstein RB, et al. The CYP2C19*17 variant is not independently associated with clopidogrel response. J Thromb Haemost 2013; 11:1640-1646

18. Sibbing D, Aradi D, Alexopoulos D, et al. Updated expert consensus statement on platelet function and genetic testing for guiding $\mathrm{P}_{2} \mathrm{Y}_{12}$ receptor inhibitor treatment in percutaneous coronary intervention. JACC Cardiovasc Interv 2019; 12:1521-1537

19. Claassens DMF, Vos GJA, Bergmeijer TO, et al. A genotype-guided strategy for oral P2 $Y_{12}$ inhibitors in primary PCI. N Engl J Med 2019; 381:1621-1631

20. Janssen PWA, Bergmeijer TO, Vos GA, et al. Tailored P2 $\mathrm{Y}_{12}$ inhibitor treatment in patients undergoing non-urgent PCI-the POPular Risk Score Study. Eur J Clin Pharmacol 2019; 75:1201-1210

21. Sibbing D, Aradi D, Jacobshagen C, et al. Guided de-escalation of antiplatelet treatment in patients with acute coronary syndrome undergoing percutaneous coronary intervention (TROPICAL-ACS): a randomised, open-label multicentre trial. Lancet 2017; 390:1747-1757

22. Neumann FJ, Sousa-Uva M, Ahlsson A, et al. 2018 ESC/EACTS guidelines on myocardial revascularization. Eur Heart J 2019; 40:87-165

23. Bergmeijer TO, Vos GJA, Claassens DMF, et al. Feasibbility and implementation of CYP2C19 genotyping in patients using antiplatelet therapy. Pharmacogenomics 2018; 19:621-628

24. Roberts JD, Wells GA, Le May MR, et al. Point-of-care genetic testing for personalisation of antiplatelet treatment (RAPID GENE): a prospective, randomised, proof-of-concept trial. Lancet 2012; 379:1705-1711

25. Kheiri B, Osman M, Abdalla A, et al. CYP2C19 pharmacogenetics versus standard of care dosing for selecting antiplatelet therapy in patients with coronary artery disease: A meta-analysis of randomized clinical trials. Catheter Cardiovasc Interv 2019; 93:1246-1252 
De-escalation of antiplatelet treatment in patients with myocardial infarction who underwent percutaneous coronary intervention: a review of the current literature

Daniel M.F. Claassens, Dirk Sibbing

Journal of Clinical Medicine 2020;9:2983

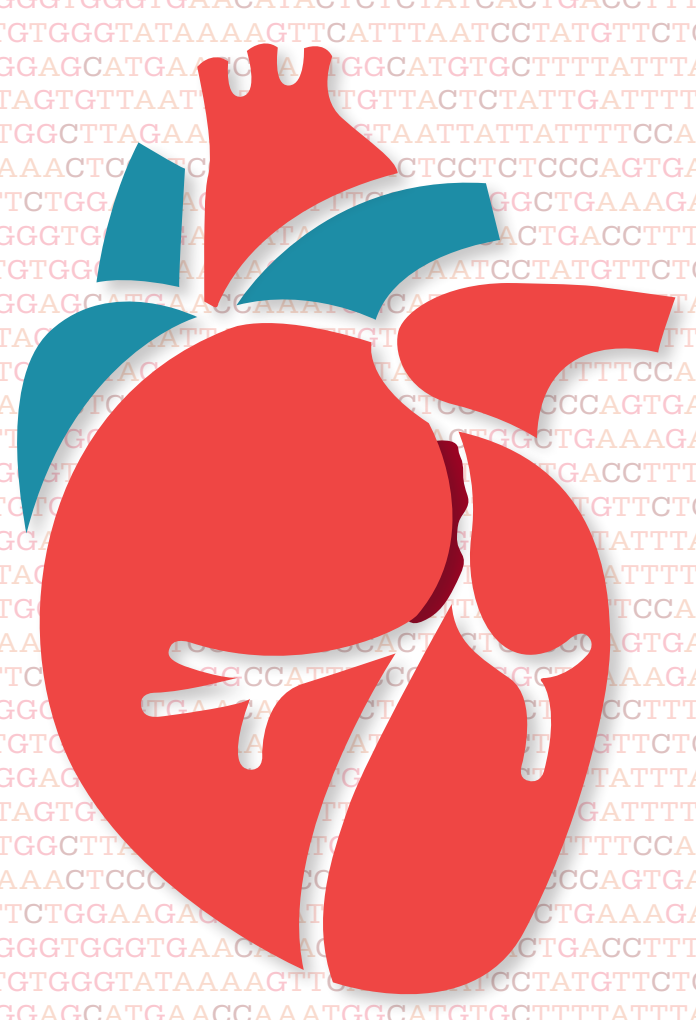




\section{Abstract}

In acute coronary syndrome (ACS) patients undergoing percutaneous coronary intervention (PCI), treatment with the $\mathrm{P}_{2} \mathrm{Y}_{12}$ inhibitors ticagrelo or prasugrel is recommended over clopidogrel due to a better efficacy, albeit having more bleeding complication. These higher bleeding rates have provoked trials investigating de-escalation from ticagrelor or prasugrel to clopidogrel in the hope of reducing bleeding without increasing thrombotic event rates. In this review, we sought to present an overview of the major trials investigating several different options for deescalation; unguided, platelet function testing- and genotype-guided. Based on these results, and on other established literature sources, such as guidelines and expert consensus papers, we provide an overview to help decide when and how to de-escalate antiplatelet therapy in ACS patients undergoing PCI.

\section{Introduction}

Patients with myocardial infarction and percutaneous coronary intervention (PCI) require dual antiplatelet therapy (DAPT) consisting of aspirin and a $\mathrm{P} 2 \mathrm{Y}_{12}$ inhibitor for at least 6 to 12 months [1,2]. In patients with myocardial infarction, potent platelet inhibition with ticagrelor or prasugrel instead of clopidogrel has been preferred by the major guidelines in the past decade. These recommendations followed after results of the Platelet Inhibition and Patient Outcomes (PLATO) and the Trial to Assess Improvement in Therapeutic Outcomes by Optimizing Platelet Inhibition With Prasugrel Thrombolysis in Myocardial Infarction 38 (TRITON-TIM 38) trials, demonstrated a reduction in thrombotic events in patients using ticagrelor and prasugrel compared to clopidogrel, and the U.S. Food and Drug Administration (FDA) added a boxed warning regarding the reduced effectiveness of clopidogrel in poor metabolizers [3,4]. However, the increased efficacy of the potent $\mathrm{P} 2 \mathrm{Y}_{12}$ inhibitors is hampered by a higher bleeding risk.

Clopidogrel is a prodrug. The active metabolite irreversibly binds to the $\mathrm{P}_{2} \mathrm{Y}_{12}$ receptor on platelets leading to reduced platelet activation [5]. Patients on clopidogrel demonstrate a wide variability in platelet reactivity and approximately $30 \%$ of the patients have an inadequate reduction in platelet reactivity measured using platelet function testing [6]. The most important enzyme in the activation process is encoded by the CYP2C19 gene. This gene has many different alleles, some of which are considered loss-of-function alleles and can be present in more than $30 \%$ of the population [7]. Patients carrying loss-of-function alleles generally show higher residual platelet reactivity and are at an increased risk for thrombotic events $[8,9]$.

\section{De-escalation of antithrombotic therapy}

The higher risk of bleeding in patients on potent $\mathrm{P} 2 \mathrm{Y}_{12}$ inhibitors remains present in the chronic treatment phase, while the greatest benefit of the potent drugs are seen early, when the risk of recurrent thrombotic events is highest $[10,11]$. De-escalation is the process of switching from the potent P2 $\mathrm{Y}_{12}$ inhibitors prasugrel or ticagrelor to weaker clopidogrel. Despite a lack of evidence supporting de-escalation, de-escalation is common in clinical practice and occurs in up to $30 \%$ of patients with myocardial infarction [12-14]. This is triggered by both clinical factors (e.g., side effects like dyspnoea or (minor) bleedings) and socioeconomic factors (e.g., higher costs of ticagrelor and prasugrel treatment) [12-14]. For instance, in the Comparison of Prasugrel and Ticagrelor in the Treatmen 
of Acute Myocardial Infarction (PRAGUE-18) trial, more than one-third of patients de-escalated to clopidogrel for economic reasons [12], while in the POPular AGE trial (including acute coronary syndrome (ACS) patients of 70 years and older), treatment adherence in the ticagrelor and prasugre group was just 53\% during the one year follow-up, mainly due to side effects and a perceived high bleeding risks [13]

This has prompted many observational studies investigating the effects of de-escalation of antiplatelet therapy [15], but in the last few years different randomized controlled trials have been published as well [16-18]. These trials investigated several different methods of de-escalation; unguided deescalation, platelet function testing (PFT)-guided de-escalation and genotype-guided de-escalation. For this review, we focus on randomized controlled trials that investigate de-escalation from ticagrelor or prasugrel to clopidogrel. Results are summarized in Table 1.

\section{Unguided de-escalation}

The Timing of Platelet Inhibition After Acute Coronary Syndrome (TOPIC) trial investigated unguided de-escalation from a potent platelet inhibitor to clopidogrel 1 month after an ACS [16]. It included 646 patients, 323 randomized to de-escalation of DAPT, and 323 to continuing poten platelet inhibition. It found a significant reduction in bleeding academic research consortium (BARC) 2 and higher bleedings (4.0\% vs. $14.9 \%$ for de-escalated and potent DAPT, respectively, HR $0.30,95 \%$ confidence interval (CI) 0.18-0.50), with no difference in thrombotic events, consisting of cardiovascular death, unplanned revascularization and stroke (9.3\% vs. $11.5 \%$, HR $0.80,95 \%$ CI $0.50-1.29)$. A pre-specified sub analysis of the TOPIC trial assessed the effect of on-treatment platelet reactivity (on prasugrel or ticagrelor) on clinical outcomes [19]. It found that de-escalation was superior regardless of initial platelet reactivity, but that patients classified as low on-treatment platelet reactivity had the highest risk of experiencing a clinical event (either bleeding or thrombotic) and benefited the most from de-escalation. Though these results seem very promising, several important limitations have to be considered. First, the trial had a small sample size. Second, there was no detailed reporting and external event adjudication on key endpoints and third, a difference in event rates, both thrombotic and bleeding, in favour of de-escalation was already seen prior to actual de-escalation.
The recently published HOST-REDUCE-POLYTECH-ACS was a randomized trial that investigated a different kind of de-escalation. It included 2338 East Asian patients with ACS and PCI who were treated with prasugrel $10 \mathrm{mg}$ daily for one month [20]. After one month, patients were randomized to either $5 \mathrm{mg}$ daily for 11 months or continued $10 \mathrm{mg}$ daily for the rest of the year. It found the reduced dose to be superior for a combined bleeding and ischemic outcome composed of all-cause death, myocardial infarction, stent thrombosis, repeat vascularization, stroke, or bleeding academic research consortium (BARC) grade 2 bleeding or higher $(7.2 \%$ vs. $10.1 \%$, hazard ratio (HR) $0.70,95 \%$ CI $0.52-0.92, p=$ 0.012 ) [20]. This was driven by a difference in BARC 2 bleeding, with no difference in ischemic outcomes. These results are promising, but there are some important limitations, mostly caused by exclusion of patient groups who cannot be treated with $10 \mathrm{mg}$ of prasugrel (history of transien ischemic attack/stroke, Age $\geq 75$ years and weight $<60 \mathrm{~kg}$ ). The mean age was only 59 years and due to the weight restriction and the low average weight of Korean women, there were very few women $(<10 \%)$. Furthermore, most ischemic events were repeat revascularization, with all other ischemic endpoints having an incidence of $<1 \%$. In addition, the mean body weight was $72 \mathrm{~kg}$ and therefore it is unsure if in other populations with much higher mean body weight, such as American and European populations, the same effect is reached. Similar to this trial, a randomized trial investigating de-escalation from a high dose to a low dose ticagrelor $(90 \mathrm{mg}$ vs. $60 \mathrm{mg}$ ) after 1 week until 1 year is ongoing (NCT04255602).

A meta-analysis from Angiolillo et al. pooled observational studies, which studied de-escalation of treatment from ticagrelor to clopidogrel for various reasons [15]. It found a rate of major adverse cardiac events (MACE) (defined as cardiovascular death, myocardial infarction and stroke) of $2 \%$, a rate of cardiovascular death of $2 \%$ and of major bleeding of $1 \%$. Contrary to these results are the results from the Switching from Clopidogrel to New Oral Antiplatelet Agents during Percutaneous Coronary Intervention (SCOPE). It found much higher rates of MACE, mostly driven by higher rates of myocardial infarction and transient ischemic attack/stroke in patients that de-escalated to clopidogrel [21] However, patients de-escalating were significantly older and more often had a history of transient ischemic attacks and stroke. In addition, the deescalation group was very small and it is unclear what the timing of events was relative to the timing of de-escalation. 

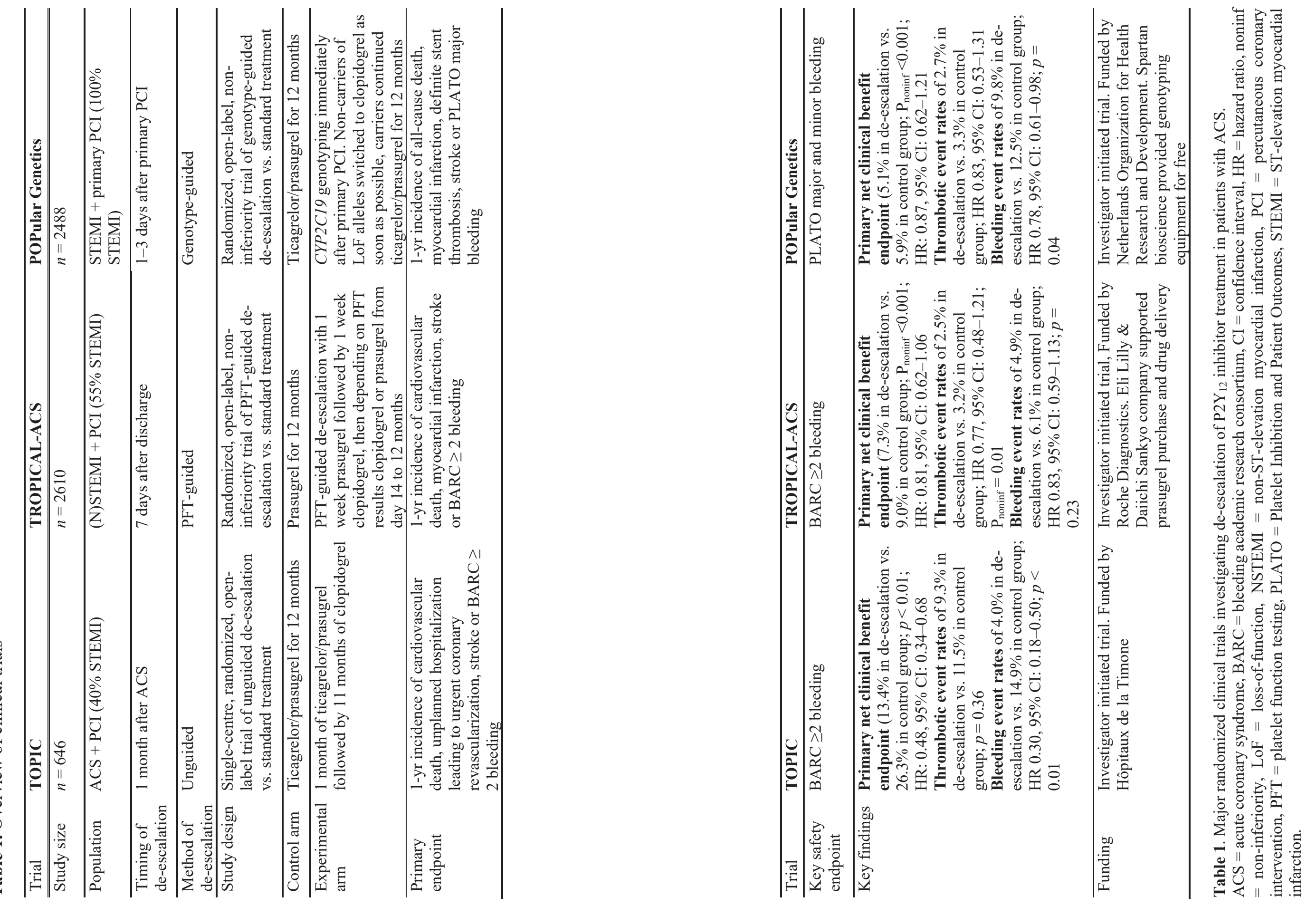


\section{Platelet function testing-guided de-escalation}

There have been many trials investigating the use of PFT to guide antithrombotic therapy. However, almost all investigated escalation to stronger or more intensified antiplatelet regimes when high platelet reactivity was found [22-24]. An exception to this was the Testing Responsiveness To Platelet Inhibition On Chronic Antiplatelet Treatment For Acute Coronary Syndromes (TROPICAL-ACS) trial, which included 2610 patients and investigated de-escalation from prasugrel to clopidogrel 7 days after hospital discharge in ST-elevation myocardial infarction (STEMI) and non-ST-elevation myocardial infarction (NSTEMI) patients (all-comers ACS cohort) who underwent PCI [17]. After 1 week of clopidogrel use, PFT was performed. Patients demonstrating high platelet reactivity switched back to prasugrel, while the other patients remained on clopidogrel until 12 months after myocardial infarction. Patients in the control arm were treated with prasugrel for 12 months.

The PFT guided group was non-inferior regarding the primary net clinical benefit outcome consisting of cardiovascular death, myocardial infarction, stroke or BARC grade 2 bleeding or higher $(7.3 \%$ vs. $9.0 \%$ in the PFTguided and standard treatment group respectively, $\mathrm{P}_{\text {non-inferiority }}<0.001 \mathrm{HR}$ $0.81,95 \% \mathrm{CI} 0.62-1.06)$ [17]. The PFT guided group was also non-inferior compared to the prasugrel treated group regarding the thrombotic outcome, defined as cardiovascular death, myocardial infarction, and stroke $(2.5 \%$ vs. $3.2 \%$ in the de-escalation and prasugrel group respectively, HR 0.77 , $\left.95 \% \mathrm{CI} 0.48-1.21, \mathrm{P}_{\text {non-inferiority }}=0.012\right)$. Of note, the trial showed numerically lower bleeding events in the guided de-escalation arm $(4.9 \%$ vs. $6.0 \%$ BARC 2 or higher bleeding for the de-escalation and prasugrel group, respectively, HR $0.82,95 \%$ CI $0.59-1.13$ ), but this difference failed to reach a level of statistical significance. A pre-specified sub-analysis of the trial assessed the impact of age clinical outcomes following PFT guided de-escalation [25]. It found that PFT guided de-escalation was associated with a significant reduction in the primary outcome in patients aged 70 and younger ( $\mathrm{HR} 0.70,95 \% \mathrm{CI} 0.51-0.96, \mathrm{p}=0.03$ ), while there was no difference in elderly patients (HR $1.17,95 \% \mathrm{CI} 0.69-2.01, \mathrm{p}=0.56$ ). This effect was mainly driven by a reduction in bleeding events in younger patients, while it must be noted that the elderly group contained only 370 patients. The TROPICAL-ACS trial also had an important limitation; the non-inferiority margin was $30 \%$, though in a post-hoc analysis noninferiority for the primary endpoint was maintained with a non-inferiority margin of $10 \%$. In addition, the PFT guided group had both numerically lower thrombotic and bleeding event rates than the standard treatment group. Further limitations included, the sole use prasugrel in the contro group and not ticagrelor, excluding patients with a history of stroke, the open-label design and the loss-to-follow-up of $4 \%$ in both arms.

In TROPICAL ACS, the Multiplate analyser was used to determine platelet inhibition [17]. Correlation between different PFTs is not very good and it is therefore unsure if results can be extrapolated to other PFTs than the Multiplate [6,26]. However, the guidelines do not endorse a specific PFT and thus leave the option on what PFT to use open.

\section{Genotype-guided de-escalation}

The last option to guide $\mathrm{P}_{2} \mathrm{Y}_{12}$ inhibitor therapy is to use CYP2C19 genetic testing. Similar to PFT, most trials and observational studies investigated escalation of therapy, including the recently published Tailored Antiplatelet Therapy Following PCI (TAILOR-PCI) [27,28]. In the primary analysis of this trial, which compared clopidogrel with ticagrelor in patients with loss-of-function alleles only, ticagrelor treatment led to a $34 \%$ reduction in ischemic events, though it missed statistical significance (HR $0.66,95 \%$ CI $0.43-1.02, \mathrm{p}=0.06$ ). This, because the trial was powered to detect a very ambitious $50 \%$ reduction in ischemic events [28]. Still, this difference is much larger than the $16 \%$ difference seen in PLATO [3]

A trial that investigated genotype-guided de-escalation was the CYP2C19 Genotype-Guided Antiplatelet Therapy in STEMI Patients-Patien Outcome after Primary PCI (POPular Genetics) trial. It investigated deescalation from potent $\mathrm{P} 2 \mathrm{Y}_{12}$ inhibitors to clopidogrel within 1 to 3 days after primary PCI in 2488 patients with ST-elevation myocardial infarction [18]. In patients randomized to the genotype-guided strategy the presence of the CYP2C19*2 and *3 allele was determined as soon as possible afte primary PCI. Non-carriers of these alleles were de-escalated to clopidogrel, while carriers remained on potent platelet inhibitors. Median time from primary PCI to de-escalation was approximately 1.5 days. Patients in the control group received standard treatment with either ticagrelor or prasugrel for 12 months. The trial found that the genotype-guided group was non-inferior to the standard-treatment group regarding net clinica benefit, defined as all-cause death, myocardial infarction, definite stent thrombosis, stroke, and PLATO major bleeding (5.1\% vs. 5.9\% for genotype-guided and standard treatment respectively, $\mathrm{P}_{\text {non-inferiority }}<0.001$, HR $0.87,95 \% \mathrm{CI} 0.62-1.21)$. It was also non-inferior regarding the thrombotic outcome, defined as cardiovascular death, myocardial infarction, definite stent thrombosis, and stroke $(2.7 \%$ vs. $3.3 \%$ for genotype-guided and standard treatment respectively, $\mathrm{HR} 0.83,95 \% \mathrm{CI}$ 
0.53-1.31). Furthermore it found that a genotype-guided strategy was superior in reducing combined PLATO major and minor bleedings $(9.8 \%$ vs. $12.5 \%$ for genotype-guided and standard treatment respectively, HR $0.78,95 \%$ CI $0.61-0.98$ ), which was mainly driven by a reduction in PLATO minor bleedings. Therefore, the genotype-guided therapy proved to be beneficial by reducing bleeding events, while not increasing thrombotic events. An important limitation of the POPular Genetics tria was the much lower than anticipated event rate. Since the non-inferiority margin was fixed $(2 \%)$, the relative margin was much greater than expected. However, similar to the PFT guided group in the TROPICALACS trial [17], patients in the genotype-guided group had numerically less thrombotic and bleeding events than patients in the standard treatmen group. Other limitations included the open-label design and that more, though rare, CYP2C19 loss-of-function alleles exist which were not tested in the trial.

\section{Summary}

Based on the currently available research, the European Society of Cardiology (ESC) guidelines provide a class IIb recommendation to use unguided, PFT, or genotyping to guide antithrombotic treatment in a subgroup of ACS patients deemed unsuitable for potent platelet inhibition [29]. An expert consensus paper concerning platelet function and genetic testing to guide $\mathrm{P} 2 \mathrm{Y}_{12}$ inhibitor treatment in PCI patients was published last year, prior to the results of the POPular Genetics trial. This consensus paper gives some suggestions as to when de-escalation in patients with myocardial infarction could be considered and when to escalate $\mathrm{P}_{2} \mathrm{Y}_{12}$ inhibitor therapy (Figure 1). In general, de-escalation should be considered in patients with a high bleeding risk (Table 2). This includes prior major bleeding, prior haemorrhagic stroke, anaemia, and clinically significant bleeding on dual-antithrombotic therapy [30]. Bleeding risk scores might also be used to help decision making, though this has never been tested in a clinical trial. Furthermore, socio-economic reasons could be a factor in deciding to de-escalate therapy. If opted to de-escalate antithrombotic therapy in a patient, choosing either PFT or genetic testing both have their advantages and disadvantages (Table 3 ). Ultimately deciding on what strategy to use will depend on the availability of the different tests and assays, the experience and logistics in the hospital and on the country and healthcare system the hospital is located in.

\section{Figure 1. Strategies for DAPT after PCI}

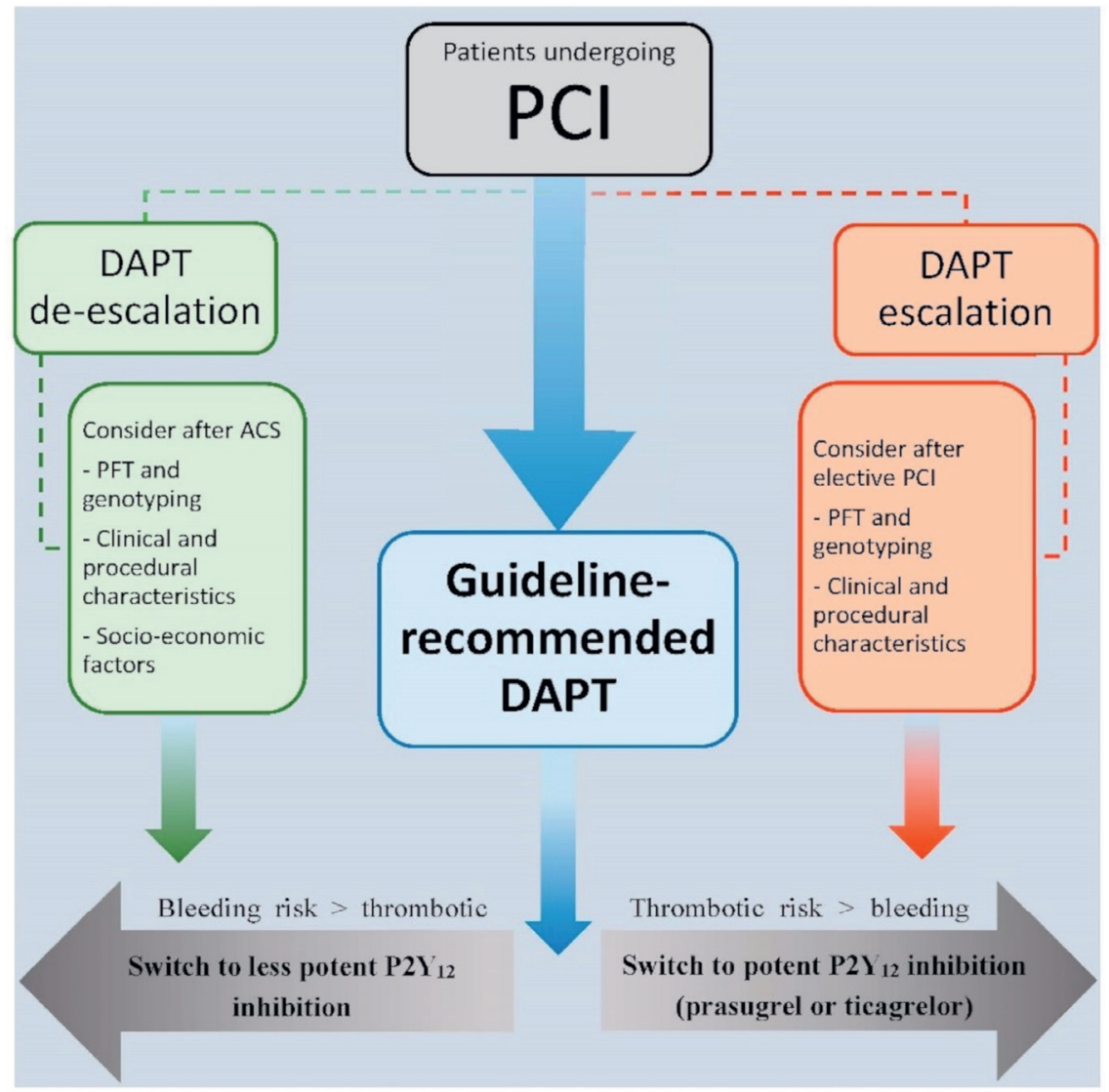

Figure 1. Strategies for dual antiplatelet therapy after PCI. The majority of patients undergoing percutaneous coronary intervention (PCI) should be treated with guideline recommended dual antiplatelet therapy (DAPT) (clopidogrel in elective PCI and ticagrelor or prasugrel in patients with acute coronary syndrome (ACS)). In elective PCI patients, an escalation strategy can be considered in some situations, when the thrombotic risk is higher than the bleeding risk. In ACS patients, de-escalation can be considered when the bleeding risk is higher than the thrombotic risk or for socio-economic considerations. 


\section{Table 2. Variables favouring de-escalation}

\begin{tabular}{|c|c|c|}
\hline \multicolumn{3}{|c|}{ Prior Major Bleeding } \\
\hline \multicolumn{3}{|c|}{ Anaemia } \\
\hline \multicolumn{3}{|c|}{ Clinically significant bleeding on potent $\mathrm{P}_{2} \mathrm{Y}_{12}$ inhibitors } \\
\hline \multicolumn{3}{|c|}{ High bleeding risk defined by bleeding risk scores } \\
\hline \multirow{2}{*}{\multicolumn{3}{|c|}{$\begin{array}{l}\text { Socio-economic factors favouring the lower costs of clopidogrel } \\
\text { Side effects on prasugrel and ticagrelor, especially dyspnoea on } \\
\text { ticagrelor }\end{array}$}} \\
\hline & & \\
\hline \multicolumn{3}{|c|}{$\begin{array}{c}\text { Need for triple treatment due to new onset atrial fibrillation or left } \\
\text { ventricular thrombus after myocardial infarction }\end{array}$} \\
\hline \multicolumn{3}{|c|}{ Table 3. Platelet function testing versus genotyping } \\
\hline & $\begin{array}{l}\text { Platelet } \\
\text { function } \\
\text { testing }\end{array}$ & Genotyping \\
\hline Availability of different assays & Yes & Yes \\
\hline Availability of point-of-care systems & Yes & Yes \\
\hline Inter-assay variability & Yes & No \\
\hline Variability of results over time & Yes & No \\
\hline Association with thrombotic events & Yes & Yes \\
\hline Association with bleeding events & Yes & Yes \\
\hline $\begin{array}{l}\text { Availability of clinical trial data on } \\
\text { guided therapy }\end{array}$ & Yes & Yes \\
\hline Feasibility in clinical practice & Yes & Yes \\
\hline $\begin{array}{l}\text { Results influenced by extra patient } \\
\text { factors }\end{array}$ & Yes & No \\
\hline Direct measure of response to therapy & Yes & No \\
\hline $\begin{array}{l}\text { Assessment of influence of both } \\
\text { genetic and non-genetic factors on } \\
\text { platelet function }\end{array}$ & Yes & No \\
\hline $\begin{array}{c}\text { Need to be performed while on } \\
\text { treatment }\end{array}$ & Yes & No \\
\hline
\end{tabular}

\section{How to de-escalate antiplatelet therapy}

After deciding to de-escalate to clopidogrel, it is possible to do this either by giving a loading dose or not. There have not been any randomized trials powered for clinical outcomes investigating this, but an expert consensus paper by Angiolillo et al. gives a recommendation based on pharmacodynamics studies. Whether or not giving a loading dose is based on the timing of de-escalation and what $\mathrm{P}_{2} \mathrm{Y}_{12}$ inhibitor is used prior to deescalation (Figure 2) [31]. When de-escalating in the early phase $(<30$ days from the index event) a $600 \mathrm{mg}$ loading dose of clopidogrel should be administered $24 \mathrm{~h}$ after the last dose of prasugrel or ticagrelor. In a subanalysis of the POPular Genetics trial, this method seemed save with no bleeding and thrombotic events in 172 patients who switched to clopidogrel within seven days after STEMI [32]. When de-escalating from ticagrelor to clopidogrel in the late phase ( $>30$ days from the index event) a $600 \mathrm{mg}$ loading dose $24 \mathrm{~h}$ after the last dose of ticagrelor is recommended while it is not recommended to use a new loading dose when switching from prasugrel to clopidogrel in the late phase. If de-escalating due to bleeding or bleeding concerns, a $75 \mathrm{mg}$ clopidogrel dose could be considered instead of a loading dose irrespective of the phase or initial P2 $\mathrm{Y}_{12}$ inhibitor [31].

\section{Conclusion and future perspective}

With a decline in ischemic events in the last decade, focus has shifted more and more towards preventing bleeding complications. In recent years, we have seen a growing body of evidence supporting de-escalation of antithrombotic therapy in patients with ACS who underwent PCI. Current guidelines offer the option to de-escalate in a subset of ACS patients who are not deemed suitable for potent platelet inhibitors. No new randomized data are expected in the near future. However, cost-effectiveness analyses and meta-analyses, including data from the latest randomized trials, might help expand the current recommendation. Subgroup analyses from currently published trials have not consistently identified patient groups that might benefit more from de-escalation. Therefore, for as long as guidelines do not give a higher recommendation to use de-escalation in broader population, it will be up to the clinician to assess whether a patient could benefit or not. 


\section{Figure 2. Recommended de-escalation strategy}

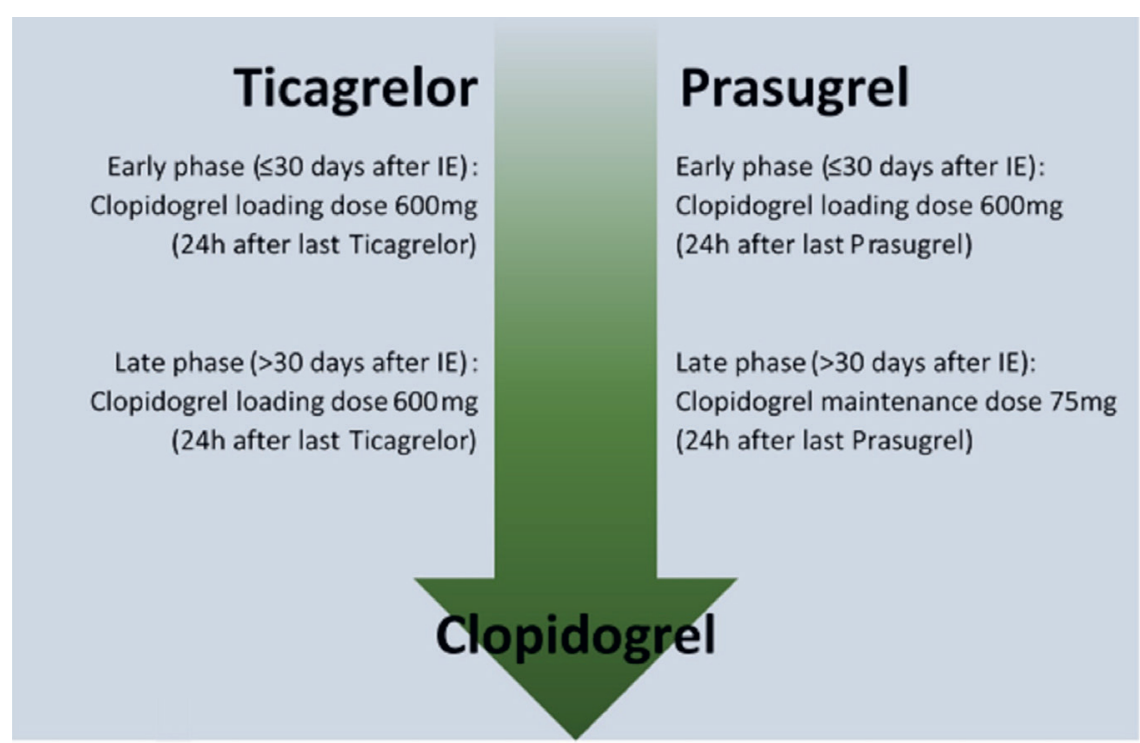

Figure 2. Recommended clopidogrel doses when de-escalating. When deescalating from ticagrelor or prasugrel to clopidogrel in the early phase ( $\leq 30$ days after the index event), a loading dose of $600 \mathrm{mg}$ should be administered $24 \mathrm{~h}$ after the last dose of the potent $\mathrm{P}_{2} \mathrm{Y}_{12}$ inhibitor. In the late phase $(>30$ days after the index event), a loading dose of $600 \mathrm{mg}$ should only be administered from ticagrelor to clopidogrel, while a maintenance dose of $75 \mathrm{mg}$ should be administered when de-escalating from prasugrel to clopidogrel. If de-escalating due to bleeding or bleeding concerns, a $75 \mathrm{mg}$ clopidogrel dose could be considered instead of a loading dose irrespective of the phase or initial $\mathrm{P} 2 \mathrm{Y}_{12}$ inhibitor.

\section{References}

1. Ibanez B, James S, Agewall, S, et al. 2017 ESC guidelines for the management of acute myocardial infarction in patients presenting with STsegment elevation: the task force for the management of acute myocardial infarction in patients presenting with ST-segment elevation of the European Society of Cardiology. Eur Heart J 2018; 39:119-177

2. Levine GN, Bates ER, Bittl JA, et al. 2016 ACC/AHA Guideline Focused Update on Duration of Dual Antiplatelet Therapy in Patients With Coronary Artery Disease. J Am Coll Cardiol 2016; 68:1082-1115

3. Wallentin L, Becker RC, Budaj A, et al. Ticagrelor versus Clopidogrel in Patients with Acute Coronary Syndromes. N Engl J Med 2009; 361:10451057

4. Wiviott SD, Braunwald E, McCabe $\mathrm{CH}$ et al. Prasugrel versus Clopidogrel in Patients with Acute Coronary Syndromes. N Engl J Med 2007; 357:20012015

5. Van Werkum J, Heestermans A, Deneer V, et al. Clopidogrel resistance: Fact and fiction. Futur Cardiol 2006; 2:215-228

6. Breet NJ, Van Werkum JW, Bouman HJ, et al. Comparison of Platelet Function Tests in Predicting Clinical Outcome in Patients Undergoing Coronary Stent Implantation. JAMA 2010; 303:754

7. Claassens, DMF, Ten Berg JM. Genotype-guided treatment of oral P2Y12 inhibitors: Where do we stand? Pharmacogenomics 2020; 21:83-86

8. Harmsze AM, Van Werkum JW, Ten Berg JM, et al. CYP2C19*2 and CYP2C9*3 alleles are associated with stent thrombosis: A case-control study. Eur Hear J 2010; 31:3046-3053

9. Angiolillo DJ, Fernández-Ortiz A, Bernardo E, et al. Variability in Individual Responsiveness to Clopidogrel. J Am Coll Cardiol 2007; 49:1505-1516

10. Antman EM, Wiviott SD, Murphy SA, et al. Early and Late Benefits of Prasugrel in Patients with Acute Coronary Syndromes Undergoing Percutaneous Coronary Intervention. J Am Coll Cardiol 2008; 51:2028-2033

11. Becker RC, Bassand JP, Budaj A, et al. Bleeding complications with the $\mathrm{P} 2 \mathrm{Y} 12$ receptor antagonists clopidogrel and ticagrelor in the PLATelet inhibition and patient Outcomes (PLATO) trial. Eur Heart J 2011; 32:29332944

12. Motovska Z, Hlinomaz O, Kala P, et al. 1-Year Outcomes of Patients Undergoing Primary Angioplasty for Myocardial Infarction Treated with Prasugrel Versus Ticagrelor. J Am Coll Cardiol 2017; 71:371-381

13. Gimbel M, Qaderdan K, Willemsen L, et al. Clopidogrel versus ticagrelor or prasugrel in patients aged 70 years or older with non-ST-elevation acute 
coronary syndrome (POPular AGE): the randomised, open-label, noninferiority trial. Lancet 2020; 395:1374-1381

14. Zettler ME, Peterson ED, McCoy LA, et al. Switching of adenosine diphosphate receptor inhibitor after hospital discharge among myocardial infarction patients: Insights from the Treatment with Adenosine Diphosphate Receptor Inhibitors: Longitudinal Assessment of Treatment Patterns and Events after Acute Coronary Syndrome (TRANSLATE-ACS) observational study. Am Heart J 2017; 183:62-68

15. Angiolillo DJ, Patti G, Chan KT, et al. De-escalation from ticagrelor to clopidogrel in acute coronary syndrome patients: A systematic review and meta-analysis. J Thromb Thrombolysis 2019; 48:1-10

16. Cuisset T, Deharo P, Quilici J, et al. Benefit of switching dual antiplatelet therapy after acute coronary syndrome: the TOPIC (timing of platelet inhibition after acute coronary syndrome) randomized study. Eur Heart J 2017; 38:3070-3078

17. Sibbing D, Aradi, D, Jacobshagen, C, et al. Guided de-escalation of antiplatelet treatment in patients with acute coronary syndrome undergoing percutaneous coronary intervention (TROPICAL-ACS): a randomised, open-label, multicentre trial. Lancet 2017; 390:1747-1757

18. Claassens DMF, Vos GJA, Bergmeijer TO, et al. A Genotype-guided strategy for oral P2Y12 inhibitors in primary PCI. N Engl J Med 2019; 381: $1621-1631$

19. Deharo P, Quilici J, Camoin-Jau L, et al. Benefit of Switching Dual Antiplatelet Therapy After Acute Coronary Syndrome According to OnTreatment Platelet Reactivity. JACC Cardiovasc Interv 2017; 10:2560-2570

20. Kim HS, Kang J, Hwang D, et al. Prasugrel-based de-escalation of dual antiplatelet therapy after percutaneous coronary intervention in patients with acute coronary syndrome (HOST-REDUCE-POLYTECH-ACS): An openlabel, multicentre, non-inferiority randomised trial. Lancet 2020; 396:10791089

21. De Luca L, D'Ascenzo F, Tarantini G, et al. Incidence and Outcome of Switching of Oral Platelet $\mathrm{P}_{2} \mathrm{Y}_{12}$ Receptor Inhibitors in Patients with Acute Coronary Syndromes Undergoing Percutaneous Coronary Intervention: The SCOPE Registry. EuroIntervention 2017; 13:459-466

22. Janssen PWA, Bergmeijer, TO, Vos, GJA, et al. Tailored $\mathrm{P}_{2} \mathrm{Y}_{12}$ inhibitor treatment in patients undergoing non-urgent $\mathrm{PCI}-$ The POPular Risk Score study. Eur J Clin Pharmacol 2019; 75:1201-1210

23. Price MJ, Berger PB, Teirstein PS, et al. Standard- vs High-Dose Clopidogrel Based on Platelet Function Testing After Percutaneous Coronary Intervention. JAMA 2011; 305:1097-1105
24. Collet JP, Cuisset, T, Rangé G, et al. Bedside Monitoring to Adjust Antiplatelet Therapy for Coronary Stenting. N Engl J Med 2012; 367:2100 2109

25. Sibbing D, Gross L, Trenk D, et al. Age and outcomes following guided deescalation of antiplatelet treatment in acute coronary syndrome patients undergoing percutaneous coronary intervention: results from the randomized TROPICAL-ACS trial. Eur Heart J 2018; 39:2749-2758

26. Larsen PD, Holley AS, Sasse A, et al. Comparison of Multiplatelet and VerifyNow platelet function tests in predicting clinical outcome in patients with acute coronary syndromes. Thromb Res 2017; 152:14-19

27. Pereira NL, Farkouh ME, So D, et al. Effect of Genotype-Guided Oral $\mathrm{P}_{2} \mathrm{Y}_{12}$ Inhibitor Selection vs Conventional Clopidogrel Therapy on Ischemic Outcomes After Percutaneous Coronary Intervention: The TAILOR-PCI Randomized Clinical trial. JAMA 2020; 324:761-771

28. Cavallari LH, Lee, CR, Beitelshees AL, et al. Multi-site investigation of outcomes with implementation of CYP2C19 genotype-guided antiplatelet therapy after percutaneous coronary intervention. JACC Cardiovasc Interv 2018; 11:181-191

29. Collet JP, Thiele H, Barbato E, et al. 2020 ESC Guidelines for the management of acute coronary syndromes in patients presenting without persistent ST-segment elevation. Eur Heart J 2021; 42:1289-1367

30. Sibbing D, Aradi D, Alexopoulos D, et al. Updated Expert Consensus Statement on Platelet Function and Genetic Testing for Guiding P2Y12 Receptor Inhibitor Treatment in Percutaneous Coronary Intervention. JACC Cardiovasc Interv 2019; 12:1521-1537

31. Angiolillo DJ, Rollini F, Storey RF, et al. International Expert Consensus on Switching Platelet P2Y12 Receptor-Inhibiting Therapies. Circulation 2017; 136:1955-1975

32. Claassens DMF, Tavenier AH, Hermanides RS, et al. Reloading when switching from ticagegrelor or prasugrel to clopidogrel within 7 days after STEMI. JACC Cardiovasc Interv 2020; 13:663-665 
Part II: Outcomes of using CYP2C19 genetic

\section{testing in clinical practice}




$$
3
$$




\section{Abstract}

Aim

A tailored antiplatelet strategy based on CYP2C19 genotype may reduce atherothrombotic and bleeding events. We describe our experience with CYP2C19 genotyping, using on-site TaqMan or Spartan genotyping or shipment to a central laboratory.

\section{Methodology}

Data from two ongoing projects were used: Popular Risk Score project (non-urgent percutaneous coronary intervention patients) and the Popula Genetics study (ST-segment elevation myocardial infarction patients). For both projects, the time to genotyping result was calculated.

\section{Results}

In the Popular Risk Score project $(\mathrm{n}=2556)$, median time from blood collection to genotyping result was 4:04 h. In the Popular Genetics study $(\mathrm{n}=1038)$, median time from randomization to genotyping result was $2: 24$

\section{Conclusion}

CYP2C19 genotyping is feasible in everyday clinical practice, both in the acute and non-acute settings.

\section{Introduction}

Clopidogrel is the $\mathrm{P} 2 \mathrm{Y}_{12}$ inhibitor of choice in patients after planned percutaneous coronary intervention (PCI). It is also still widely used in patients with acute coronary syndrome (ACS), although the European Society of Cardiology guidelines recommend the more potent $\mathrm{P} 2 \mathrm{Y}_{12}$ inhibitors prasugrel and ticagrelor in those patients [1,2]. The highe efficacy with regard to the prevention of atherothrombotic events when using prasugrel or ticagrelor, however, comes at the cost of an increased bleeding risk and higher drug costs $[3,4]$.

Clopidogrel is subject to a wide interpatient variation in drug response, influenced by genetic factors, clinical factors and drug-drug interactions (i.e., calcium channel blockers, certain proton pump inhibitors such as omeprazole and esomeprazole or St John's Wort) [5-7]. Clopidogrel is a prodrug that needs to be activated by hepatic CYP450 enzymes to become effective [8]. Patients who are carrier of one or more CYP2C19 loss-offunction alleles have a lower active metabolite level [9-12], higher leve of platelet reactivity and an increased risk for recurrent atherothrombotic events, in particular in the patients with the highest thrombotic risk [13$16]$.

In contrast, ticagrelor does not need biotransformation to become effective, and, although CYP2C19 is involved in prasugrel metabolism as well, the CYP2C19 genotype does not have a clinically relevant influence on prasugrel efficacy $[17,18]$. Non-functional $C Y P 2 C 19$ polymorphisms are no rare phenomena, while for Caucasian patients, the allele frequency of CYP 2 C $19 * 2 \quad(r \mathrm{~s} 4244285,681 \mathrm{G}>\mathrm{A}) \quad$ is approximately $15 \%$ and approximately $0.4 \%$ for $C Y P 2 C 19 * 3$ (rs4986893, 636G $>A$ ). In Asian patients, those numbers are even higher (respectively, $29-35 \%$ and 2.4 $8.9 \%)[19]$.

The finding of a diminished clopidogrel efficacy in patients carrying a CYP2C19 loss-of-function allele has led to the addition of a black-box warning to the clopidogrel label by the US FDA, who warned physicians that an alternative antiplatelet drug should be considered in $C Y P 2 C 19 * 2$ homozygote (poor-metabolizer) patients [20]. Furthermore, the EMA gave a warning for a diminished effect in CYP2C19 poor-metabolizer patients [21]. The Clinical Pharmacogenetics Implementation Consortium (CPIC) guideline as well as the Dutch Pharmacogenetics Working Group guideline state that an alternative antiplatelet agent for clopidogrel should be considered in ACS and PCI patients who are intermediate or poor metabolizer for CYP2C19 [19,22]. 
Randomized trials testing personalized treatment strategies based on CYP2C19 genotype in patients treated with $\mathrm{P}_{2} \mathrm{Y}_{12}$ inhibitors are ongoing, such as the Popular Genetics study in patients with ST-segment elevation myocardial infarction (STEMI) undergoing primary PCI [23], and the Tailored Antiplatelet Initiation to Lessen Outcomes Due to Decreased Clopidogrel Response after Percutaneous Coronary Intervention (TAILOR-PCI) study in PCI patients (ClinicalTrials.gov NCT01742117). Those personalized treatment strategies are based on the hypothesis that clopidogrel efficacy is comparable to ticagrelor or prasugrel in the subgroup of normal metabolizer $(* 1 / * 1)$ patients, while the bleeding risk might actually be lower $[4,11,16,24]$. Therefore, treating CYP2C19 norma metabolizer ACS patients with clopidogrel, although not recommended by current non-STEMI and STEMI guidelines, might reduce bleeding risk and drug costs, without an increase in thrombotic events [1,2]. However, to make these personalized medicine strategies a success, CYP2C19 genotyping has to be feasible in everyday clinical practice. In this paper, the feasibility of CYP2C19 genotyping is studied, using the data of two projects in which CYP2C19 genotyping is used to tailor antiplatelet treatment.

\section{Methods}

\section{Popular risk score project}

The Popular Risk Score project was a single-centre prospective registry that included consecutive patients in whom non-urgent PCI with stent implantation was performed. A risk score was calculated in all patients, consisting of platelet reactivity testing using the VerifyNow P2 $\mathrm{Y}_{12}$ assay (Accriva Diagnostics, CA, USA), CYP2C19*2 and *3 genotyping using the TaqMan StepOnePlus assay, and clinical variables (diabetes, adjoined stent length $>30 \mathrm{~mm}$ and left ventricular ejection fraction $<30 \%$ ). The risk score and associated platelet function and genetic testing were performed as part of routine clinical care. Patients with a high-risk score were treated with prasugrel for 1 year, while patients with a low risk score were treated with clopidogrel for 1 year [25]

\section{Implementation}

In all patients planned for PCI, one ethylenediaminetetraacetic acid (EDTA) tube with blood was collected using venepuncture before the procedure to perform $C Y P 2 C 19$ genotyping. When a blood sample could not be obtained before PCI, a blood sample was collected during PCI from the arterial catheter or after PCI using venepuncture (in a small proportion of patients). The technicians of the pharmacogenetics laboratory collected the samples on a routine basis three-times a day (at 8:00, 10:00 and 14:00 h) and performed genotyping for $C Y P 2 C 19 * 2$ and *3. Genotyping results were reported in the hospital electronic patient file. After the PCI, the medical physician responsible for the Cath lab reviewed all results and decided, based on the calculated Popular Risk Score, if the antiplatelet therapy had to be changed.

\section{Validation}

CYP2C19*2 and $* 3$ genotyping was performed by using a real-time PCR technique with TaqMan Genotyping MasterMix and TaqMan Drug Metabolism Genotyping assay performed on the StepOnePlus (ThermoFisher, MA, USA). The assays were validated by the pharmacogenetics laboratory before applying the test in routine patien care. As part of the validation process, selected samples with different genotypes based on the results of the TaqMan assay were also sequenced. Genotypes obtained by both methods were equal.

\section{POPular Genetics study}

The POPular Genetics study (ClinicalTrials.gov, NCT01761786) is an ongoing international multicentre, open label, randomized clinical trial consisting of patients with STEMI in whom primary PCI was performed. The rationale and design of this study have been published earlier [23]. In short, patients were randomized to a control group, treated with ticagrelor or prasugrel for 1 year, or an intervention group in which $C Y P 2 C 19 * 2$ and $* 3$ genotyping was performed. Normal metabolizer patients $(* 1 / * 1)$ were treated with clopidogrel, while patients carrying one or two loss-offunction alleles $(* 2$ or $* 3)$ were treated with prasugrel or ticagrelor. CYP2C19*17 was not tested. Different CYP2C19 genotyping strategies were used in different study sites: on-site testing using the TaqMan StepOnePlus assay, on-site genotyping using the Spartan RX Point-of-Care (POC) device or shipment to a central laboratory using postal or courier service for TaqMan StepOnePlus genotyping. In the central laboratory (which is the same laboratory in which genotyping is performed for the Popular Risk Score project), CYP2C19 genotyping is routinely performed three-times daily on working days (Monday to Friday) and one time daily on Sunday. No routine testing was performed on Saturday.

\section{Implementation}

An EDTA blood sample was collected from all study patients and sent to the central laboratory. Genotyping for CYP $2 C 19 * 2$ and $* 3$ was performed in patients randomized to the genotyping arm, after written informed consent was obtained. During the course of this study, a Spartan RX genotyping device was made available by the company in seven centres for use in this study. A buccal swab was collected and directly analysed in the 
genotyping device in these centres. In one centre, the Spartan RX genotyping was performed by laboratory personnel; in the other centres Spartan RX genotyping was performed by a local study investigator or a study nurse. Users were trained by the Spartan company personnel in person or via a video conference session. The device could be placed in the clinical chemistry laboratory, catheterization laboratory or coronary care unit. From six centres without on-site genotyping capability, blood samples were shipped to the study's central laboratory for TaqMan genotyping. Results from central laboratory testing were reported in the local electronic patient file and sent to the referring hospital by email by the coordinating study investigator. In one centre, on-site TaqMan genotyping was used. This centre was also the central laboratory site. Over time, centres could change their genotyping strategy - that is, when a Spartan RX device came available. All genotyping results were sent to the patient's general practitioner, treating cardiologist and local pharmacy.

\section{Validation}

Because central laboratory testing was performed in the same laboratory where the Popular Risk Score genotyping was performed, validation was equal to the method described above. For confirmation purposes, 199 patients tested with the Spartan RX device were also genotyped using TaqMan genotyping on the blood sample sent to the central laboratory.

\section{Statistical analysis}

Statistical analyses were performed using SPSS software (v24.0, IBM, USA). For patients in the Popular Risk Score project, the time between blood collection and reporting of the genotyping test result was calculated. For patients in the Popular Genetics study, the time between randomization and reporting of the genotyping test result was calculated. The time between blood collection and test result was not considered the most relevant measure to study the feasibility of the different genotyping methods, because of the design of the study: genotyping could only be performed after informed consent was obtained and randomization was performed.

\section{Results}

\section{Popular risk score}

Between July 2010 and July 2016, 2556 patients were genotyped for the purpose of calculating the Popular Risk Score. Those patients were on average 65 years of age ( \pm 10.7 years) and $74.3 \%$ of the patients were male. Distribution of CYP2C19 genotype is shown in Figure 1A. Median time between blood collection and genotyping result was 4:04 h ([interquartile range (IQR) 2:27, 16:57], range 1:16-96:51 h) (Figure 2). For 49.1\% of patients, the $C Y P 2 C 19$ genotyping result was made available to the treating medical physician within $4 \mathrm{~h}$ after PCI, in $71.9 \%$ at the same day PCI was performed, in $94.5 \%$ within $24 \mathrm{~h}$ and in $96.9 \%$ within $48 \mathrm{~h}$ after PCI. In almost all patients in whom genotyping result took more than $48 \mathrm{~h}$, the blood sample was collected at Friday afternoon, after the last genotyping round. In those patients, genotyping results were available the following Monday morning. In the Popular Risk Score cohort, 2199 patients $(86.0 \%)$ received coronary stent implantation. In those patients, data of $\mathrm{P}_{2} \mathrm{Y}_{12}$ inhibitor use following the PCI were available in 1449 patients $(65.9 \%)$. In total, 991 patients $(68.4 \%)$ had a low risk score and were advised to use clopidogrel. This advice was followed in 988 patients (99.7\%); one patient had an allergy for clopidogrel, one patients received coronary artery bypass grafting after which the $\mathrm{P}_{2} \mathrm{Y}_{12}$ inhibitor was stopped, and for one patient it was not clear why the risk score advice was not followed. In total, 456 patients $(31.5 \%)$ were advised to use prasugrel or ticagrelor, which was followed in 405 patients $(88.8 \%)$; three patients received clopidogrel because of a high bleeding risk, two because of concomitant use of acenocoumarol, two patients had a contraindication for prasugrel (previous stroke) and in 44 patients, it was not clear why the risk score advice was not followed.

\section{POPular Genetics study}

All patients included between July 2014 (study initiation) and 8th December 2017 in the POPular Genetics study, and randomized to the genotyping arm, were selected. This selection contained 1283 patients. Because it is an ongoing trial, data were not yet entered in the electronic case record form for a part of the patient cohort. The electronic case record form was complete with data describing the method of genotyping and the time between randomization and genotyping result in 1038 patients $(80.9 \%)$ from nine study sites. These patients were on average 61.9 years of age ( \pm 11.2 years) and $74.3 \%$ of the patients were male. On-site TaqMan genotyping was used by one study site (487 patients), on-site Spartan RX genotyping by seven study sites (411 patients) and shipment to the central laboratory was used by six study sites (140 patients). Distribution of CYP2C19 genotype is shown in Figure 1B. The median time between randomization and genotyping result was $2: 24 \mathrm{~h}$ ([IQR 1:46, 12:53], range 0:30-13:32 h); 2:04 h $(1: 44,3: 58)$ for in-hospital TaqMan genotyping, $2: 16$ h $(1: 38,4: 29)$ for Spartan RX genotyping and 52:32 h (28:16, 80:13) for central laboratory testing (Figure $2 \&$ Supplementary Materials). For all patients in the database tested with the Spartan RX device, the test results were inconclusive in 39 patients $(8.0 \%)$. This was solved by 
shipment to the central laboratory for TaqMan genotyping (30 patients), or repeated Spartan RX testing (two patients). In seven patients, no further genotyping was performed; in five patients ticagrelor was continued, one patient was switched to clopidogrel because a vitamin $\mathrm{K}$ antagonist was started and the discharge medication of the remaining patient was unknown. In one patient, the Spartan RX result did not match the TaqMan result, which was most likely due to contamination of the Spartan RX sample. Repeated testing of the same patient gave results matching the central laboratory result.

\section{Figure 1. CYP2C19 allele frequency}

A
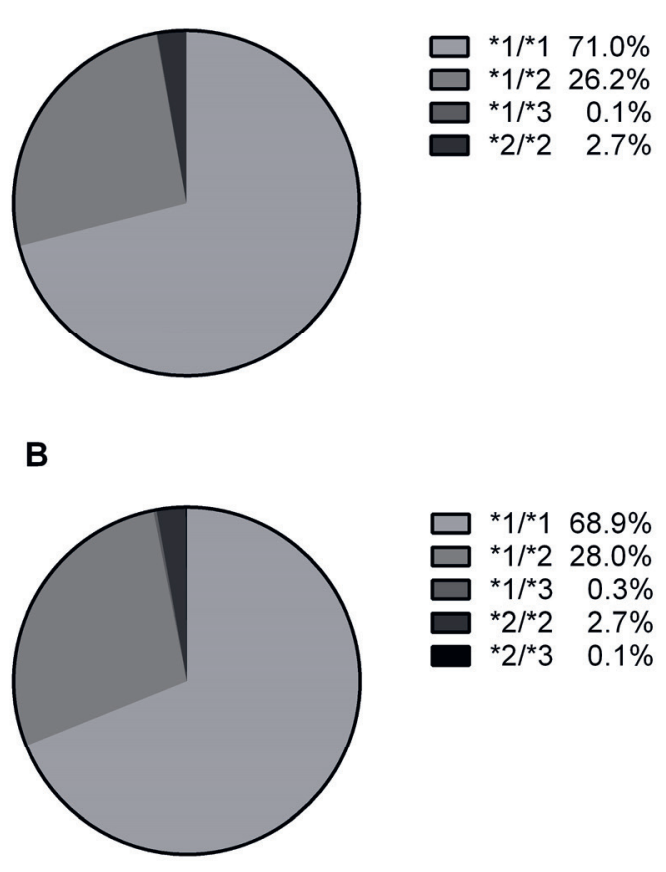

Figure 1. (A) CYP2C19 allele frequency in the Popular Risk Score project; (B) CYP2C19 allele frequency in the POPular Genetics study.
Figure 2. Time to genotyping results

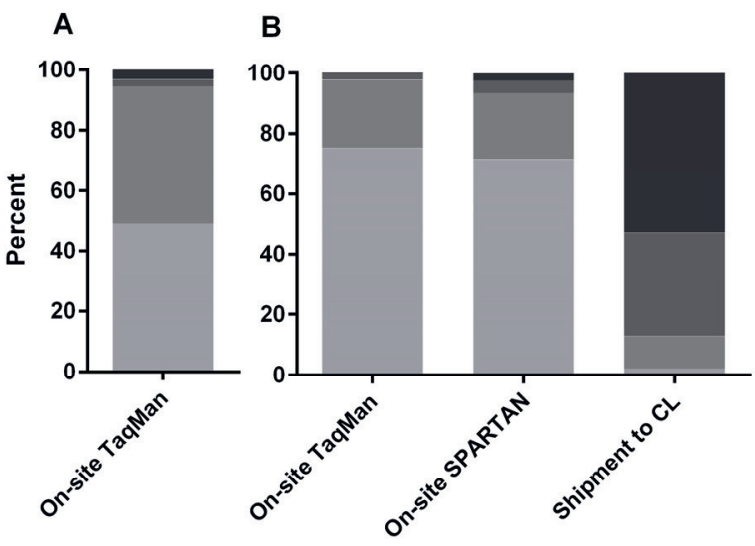

48 hours or more
within 48 hours

within 48 hours

within 4 hours

2. Time between blood collection (A) or randomization (B) and CYP2C19 genotyping results, for each genotyping method. (A) Popular Risk Score project; (B) Popular Genetics study. CL: Central laboratory.

\section{Discussion}

In this analysis, we evaluated the feasibility of different strategies of CYP2C19 genotyping, used to tailor antiplatelet therapy in patients admitted with STEMI or undergoing a planned PCI. We conclude that it is feasible to have genotyping results available within $24-48 \mathrm{~h}$ in the majority of patients, using TaqMan StepOnePlus genotyping or the Spartan RX POC device. As expected, shipment to a central laboratory takes more time than on-site genotyping. The use of the Spartan RX POC device has advantages over TaqMan genotyping. First, results can be available within $1 \mathrm{~h}$ after collection of a buccal swab. This makes it possible for patients undergoing planned PCI to have genotyping results available before same day discharge. Furthermore, in the acute setting of myocardial infarction antiplatelet therapy might be adjusted shortly after hospital arrival, even during weekend-, evening- or night-time hours. Second, the use of a buccal swab is more patient friendly compared with venepuncture for an additional blood sample. Third, although the test must be performed by trained personnel, the POC device can be situated outside the laboratory in example, in the Cath lab or on the CCU. Another disadvantage for 
TaqMan genotyping is that laboratory personnel performing the test have to be trained to a higher level and a certified laboratory has to be available

There are also disadvantages related to POC testing. First, although confirmation studies show a good correlation between Spartan RX and laboratory-based genotyping [26,27], in one patient the Spartan test result did not match with TaqMan genotyping result, and in $8 \%$ of patients in our cohort the test result was inconclusive on the first attempt. Although this problem might be solved at least for some part with more extensive training, laboratory based testing has to be available for backup. Second, in high-volume centres with a well-organized laboratory for genotyping, batch-wise testing using, in other words, TaqMan might be cost-efficient in comparison to POC genotyping. This should be analysed in more detail Third, when TaqMan genotyping is used, more SNP's and patients can be tested simultaneously, while the Spartan RX is limited to CYP 2 C19*2, *3 and $* 17$, for one patient at a time per genotyping device. Fourth, for lowrisk patients - in other words, patients planned for elective PCI - it might not be necessary to have genotyping results available the same day and genotyping could actually be performed days or weeks in advance, in which batch wise laboratory-based testing can be a good alternative to POC testing.

CYP2C19 genotyping has additional costs. Current list prices for the SPARTAN RX system at time of publication are US\$200 (€162) per tes kit, including testing for $* 2, * 3$ and $* 17$, and US $\$ 15,000(€ 12,173)$ for the SPARTAN RX POC device. The costs for laboratory-based testing are estimated at $€ 80$ per patient, including reagent and personnel costs, but excluding equipment. The costs of laboratory based testing, however, may vary substantially per country and per laboratory site, in other words, with the number of tests performed. Nevertheless, a tailored antiplatelet strategy might be cost-effective, based on the much lower drug costs of generic clopidogrel ( $€ 14.40$ per year in The Netherlands) compared with prasugre and ticagrelor (€722 and $€ 877$ per year, respectively) [28]. This difference might be even bigger if a tailored approach would be able to reduce clinical events or give a gain in quality-adjusted life-years. In an observationa study comparing $32 \mathrm{CYP} 2 \mathrm{C} 19$ poor-metabolizer patients treated with clopidogrel compared with 41 poor-metabolizer patients treated with prasugrel in The Netherlands, a significant lower number of events was seen in the prasugrel-treated patient group [29]. Non-randomized trials showed that personalized antiplatelet treatment based on CYP2C19 genotype might be able to reduce cardiovascular event rates and bleeding events $[16,30]$. Furthermore, several simulation studies showed superiority in cost-effectiveness for a $C Y P 2 C 19$ genotype guided strategy compared with the use of prasugrel or ticagrelor, in both ACS and PCI patients [3133]. In the ongoing Popular Genetics study, cost-effectiveness will be studied as a co-primary end point [23]

The putative gain-of-function allele $C Y P 2 C 19 * 17$ has been associated with a lower platelet reactivity and an increased risk of bleeding [34,35]. In both the Popular Risk Score project and the Popular Genetics study, the *17 allele was not tested because it would not have had consequences for the treatment advice in those studies. Patients with $* 1 / * 1, * 1 / * 17$ or $* 17 / * 17$ genotype would all be considered good responders and treated with clopidogrel, while patients with $* 2 / * 17$ are classified as intermediate metabolizers and therefore treated with prasugrel or ticagrelor [34]

With genotyping becoming part of routine care, it is important to realize that the results might affect other treatments than just antiplatelet therapy, as it is involved in the metabolism of selective serotonin reuptake inhibitors, tricyclic antidepressants, proton pump inhibitors and voriconazole [22,36-38]. Therefore, it is important that the genotyping results are reported in the electronic patient file and that protocols that describe how to handle possible genotype-drug interactions, made in collaboration with the clinical pharmacist, are available. Genotyping results should also be shared with the local pharmacy and the general practitioner [22]

\section{Limitations}

The aim of this paper was to study the feasibility of different CYP2C19 genotyping strategies in everyday practice, not to compare the time it takes to run the tests itself. When using the Spartan RX POC device, the time between collection of the buccal swab and test result will be around $1 \mathrm{~h}$, while DNA extraction and TaqMan genotyping will take less than $2 \mathrm{~h}$ in total, when a blood sample is available.

\section{Conclusion}

It is feasible to perform CYP2C19 genotyping within $24-48 \mathrm{~h}$ using on-site TaqMan genotyping or the Spartan RX POC device, making personalized medicine in antiplatelet therapy, based on pharmacogenetic data, feasible for everyday practice. 


\section{References}

1. Ibanez B, James S, Agewall S, et al. 2017 ESC guidelines for the management of acute myocardial infarction in patients presenting with STsegment elevation. The Task Force for the management of acute myocardial infarction in patients presenting with ST-segment elevation of the European Society of Cardiology (ESC). Eur Heart J 2018; 39:119-177

2. Roffi M, Patrono C, Collet JP, et al. 2015 ESC guidelines for the management of acute coronary syndromes in patients presenting withou persistent ST-segment elevation. Task Force for the Management of Acute Coronary Syndromes in Patients Presenting without Persistent ST-Segment Elevation of the European Society of Cardiology (ESC). Eur Heart J 2016; Elevation of

3. Wallentin L, Becker RC, Budaj A, et al. Ticagrelor versus clopidogrel in patients with acute coronary syndromes. N Engl J Med 2009; 361:1045-105

4. Wiviott SD, Braunwald E, McCabe $\mathrm{CH}$, et al. Prasugrel versus clopidogre in patients with acute coronary syndromes. N Engl J Med 2007; 357:20012015

5. Siller-Matula JM, Lang I, Christ G, et al. Calcium-channel blockers reduce the antiplatelet effect of clopidogrel. J Am Coll Cardiol 2008; 52:1557-1563

6. Gilard M, Arnaud B, Cornily JC, et al. Influence of omeprazole on the antiplatelet action of clopidogrel associated with aspirin. The randomized, double-blind OCLA (Omeprazole Clopidogrel Aspirin) study. J Am Coll Cardiol 2008; 51:256-260

7. Siller-Matula JM, Trenk D, Krahenbuhl S, et al. Clinical implications of drug-drug interactions with P2Y12 receptor inhibitors. J Thromb Haemost 2014; 12:2-13

8. Kazui M, Nishiya $\mathrm{Y}$, Ishizuka $\mathrm{T}$, et al. Identification of the human cytochrome P450 enzymes involved in the two oxidative steps in the bioactivation of clopidogrel to its pharmacologically active metabolite. Drug Metab Dispos 2010; 38:92-99

9. Hulot JS, Bura A, Villard E, et al. Cytochrome P450 2C19 loss-of-function polymorphism is a major determinant of clopidogrel responsiveness in healthy subjects. Blood 2006; 108:2244-2247

10. Shuldiner AR, O'Connell JR, Bliden KP, et al. Association of cytochrome P450 2C19 genotype with the antiplatelet effect and clinical efficacy of clopidogrel therapy. JAMA 2009; 302:849-857

11. Mega JL, Close SL, Wiviott SD, et al. Cytochrome P-450 polymorphisms and response to clopidogrel. N Engl J Med 2009; 360:354-362

12. Brandt JT, Payne CD, Wiviott SD, et al. A comparison of prasugrel and clopidogrel loading doses on platelet function. magnitude of platelet inhibition is related to active metabolite formation. Am Heart J 2007; 153:66.e9-66.e16

13. Mega JL, Simon T, Collet JP, et al. Reduced-function CYP2C19 genotype and risk of adverse clinical outcomes among patients treated with clopidogrel predominantly for PCI. A meta-analysis. JAMA 2010; 304:1821-1830
14. Harmsze AM, van Werkum JW, Ten Berg JM, et al. CYP2C19*2 an CYP2C $9 * 3$ alleles are associated with stent thrombosis. A case-contro study. Eur Heart J 2010; 31:3046-3053

15. Sorich MJ, Rowland A, McKinnon RA, et al. CYP2C19 genotype has a greater effect on adverse cardiovascular outcomes following percutaneous coronary intervention and in Asian populations treated with clopidogrel: a meta-analysis. Circ Cardiovasc Genet 2014; 7:895-902

16. Cavallari LH, Lee CR, Beitelshees AL, et al. Multisite investigation of outcomes with implementation of CYP2C19 genotype-guided antiplatelet therapy after percutaneous coronary intervention. JACC Cardiovasc Interv 2017; 11:181-191

17. Wallentin L, James S, Storey RF, et al. Effect of CYP2C19 and ABCB1 single nucleotide polymorphisms on outcomes of treatment with ticagrelor versus clopidogrel for acute coronary syndromes. a genetic substudy of the PLATO trial. Lancet 2010; 376:1320-1328

18. Brandt JT, Close SL, Iturria SJ, et al. Common polymorphisms of CYP2C19 and $\mathrm{CYP} 2 \mathrm{C} 9$ affect the pharmacokinetic and pharmacodynamic response to clopidogrel but not prasugrel. J Thromb Haemost 2007; 5:2429-2436

19. Scott SA, Sangkuhl K, Stein CM, et al. Clinical Pharmacogenetics Implementation Consortium guidelines for CYP2C19 genotype and clopidogrel therapy. 2013 update. Clin Pharmacol Ther 2013; 94:317-323

20. Holmes DR Jr, Dehmer GJ, Kaul S, et al. ACCF/AHA clopidogrel clinica alert: approaches to the FDA 'boxed warning': a report of the American College of Cardiology Foundation Task Force on clinical expert consensus documents and the American Heart Association endorsed by the Society for Cardiovascular Angiography and Interventions and the Society of Thoracic Surgeons. J Am Coll Cardiol 2010; 56:321-341

21. European Medicines Agency (EMA). Summary of product characteristics (EPAR) - Clopidogrel (Plavix). 27th November 2017. www.ema.europa.eu/docs/en $\quad$ GB/document $\quad$ library/EPARPww.ema.europa.eu/docs/en GB/document

22. Swen JJ, Nijenhuis M, de Boer A, et al. Pharmacogenetics. from bench to byte - an update of guidelines. Clin Pharmacol Ther 2011; 89:662-673

23. Bergmeijer TO, Janssen PW, Schipper JC, et al. CYP2C19 genotype-guided antiplatelet therapy in ST-segment elevation myocardial infarction patientsrationale and design of the patient outcome after primary PCI (Popular) Genetics study. Am. Heart J 2014; 168:16-22.e1

24. Sorich MJ, Vitry A, Ward MB, et al. Prasugrel vs. clopidogrel for cytochrome P450 2C19-genotyped subgroups: integration of the TRITONTIMI 38 trial data. J Thromb Haemost 2010; 8:1678-1684

25. Janssen PWA. Selective intensification of P2Y12 inhibitor therapy based on platelet reactivity, CYP2C19 genotype and clinical risk factors in non-urgent PCI patients. In: EuroPCR - Hot Line Session Antithrombotic Strategies. European Society of Cardiology, Paris, France, 21-25 (2015) 
26. Wirth F, Zahra G, Xuereb RG, et al. Comparison of a rapid point-of-care and two laboratory-based CYP2C19*2 genotyping assays for personalisation of antiplatelet therapy. Int J Clin Pharm 2016; 38:414-420

27. Roberts JD, Wells GA, Le May MR, et al. Point-of-care genetic testing for personalisation of antiplatelet treatment (RAPID GENE). A prospective, randomised, proof-of-concept trial. Lancet 2012; 379:1705-1711

28. Zorginstituut Nederland. www.medicijnkosten.nl/

29. Deiman BA, Tonino PA, Kouhestani K, et al. Reduced number of cardiovascular events and increased cost-effectiveness by genotype-guided antiplatelet therapy in patients undergoing percutaneous coronary interventions in The Netherlands. Neth Heart J 2016; 24:589-599

30. Sanchez-Ramos J, Davila-Fajardo CL, Toledo Frias P, et al. Results of genotype-guided antiplatelet therapy in patients who undergone percutaneous coronary intervention with stent. Int J Cardiol 2016; 225:289295

31. Borse MS, Dong OM, Polasek MJ, et al. CYP2C19-guided antiplatelet therapy: a cost-effectiveness analysis of 30-day and 1-year outcomes following percutaneous coronary intervention. Pharmacogenomics 2017; 18:1155-1166

32. Jiang M, You JH. CYP2C19 LOF and GOF-guided antiplatelet therapy in patients with acute coronary syndrome: a cost-effectiveness analysis. Cardiovasc Drugs Ther 2017; 31:39-49

33. Kazi DS, Garber AM, Shah RU, et al. Cost-effectiveness of genotype-guided and dual antiplatelet therapies in acute coronary syndrome. Ann Intern Med 2014; 160:221-232

34. Harmsze AM, van Werkum JW, Hackeng CM, et al. The influence of CYP2C19*2 and *17 on on-treatment platelet reactivity and bleeding events in patients undergoing elective coronary stenting. Pharmacogenet Genomics 2012; 22:169-175

35. Sibbing D, Koch W, Gebhard D, et al. Cytochrome 2C19*17 allelic variant platelet aggregation, bleeding events, and stent thrombosis in clopidogreltreated patients with coronary stent placement. Circulation 2010; 121:512518

36. Hicks JK, Swen JJ, Thorn CF, et al. Clinical Pharmacogenetics Implementation Consortium guideline for CYP2D6 and CYP2C19 genotypes and dosing of tricyclic antidepressants. Clin Pharmacol Ther 2013; 93:402-408

37. Hicks JK, Bishop JR, Sangkuhl K, et al. Clinical Pharmacogenetics Implementation Consortium (CPIC) guideline for CYP2D6 and CYP2C19 genotypes and dosing of selective serotonin reuptake inhibitors. Clin Pharmacol Ther 2015; 98:127-134

38. Moriyama B, Obeng AO, Barbarino J, et al. Clinical Pharmacogenetics Implementation Consortium (CPIC) guidelines for CYP2C19 and voriconazole therapy. Clin Pharmacol Ther 2017; 102:45-51

\section{Supplementary appendix}


Table 1: Time between randomization and genotyping result per centre (POPular Genetics study)

\begin{tabular}{|c|c|c|c|c|c|c|c|}
\hline & & & $\begin{array}{c}<2 \\
\text { hours } \\
\end{array}$ & $\begin{array}{l}<24 \\
\text { hours } \\
\end{array}$ & $\begin{array}{c}<48 \\
\text { hours } \\
\end{array}$ & $\begin{array}{l}>=48 \\
\text { hours }\end{array}$ & \\
\hline \multirow{4}{*}{1} & \multirow{2}{*}{$\begin{array}{c}\text { Shipment } \\
\text { to CL }\end{array}$} & Count & & 4 & 3 & & 7 \\
\hline & & $\%$ & & $57.1 \%$ & $42.9 \%$ & & $100.0 \%$ \\
\hline & \multirow{2}{*}{ Total } & Count & & 4 & 3 & & 7 \\
\hline & & $\%$ & & $57.1 \%$ & $42.9 \%$ & & $100.0 \%$ \\
\hline \multirow{6}{*}{2} & \multirow{2}{*}{$\begin{array}{l}\text { On-site } \\
\text { TaqMan }\end{array}$} & Count & 366 & 110 & 10 & 1 & 487 \\
\hline & & $\%$ & $75.2 \%$ & $22.6 \%$ & $2.1 \%$ & $0.2 \%$ & $100.0 \%$ \\
\hline & \multirow{2}{*}{ Spartan } & Count & 76 & 6 & 0 & 0 & 82 \\
\hline & & $\%$ & $92.7 \%$ & $7.3 \%$ & $0.0 \%$ & $0.0 \%$ & $100.0 \%$ \\
\hline & \multirow{2}{*}{ Total } & Count & 442 & 116 & 10 & 1 & 569 \\
\hline & & $\%$ & $77.7 \%$ & $20.4 \%$ & $1.8 \%$ & $0.2 \%$ & $100.0 \%$ \\
\hline \multirow{6}{*}{3} & \multirow{2}{*}{$\begin{array}{c}\text { Shipment } \\
\text { to CL }\end{array}$} & Count & 0 & 3 & 11 & 3 & 17 \\
\hline & & $\%$ & $0.0 \%$ & $17.6 \%$ & $64.7 \%$ & $17.6 \%$ & $100.0 \%$ \\
\hline & \multirow{2}{*}{ Spartan } & Count & 156 & 52 & 4 & 3 & 215 \\
\hline & & $\%$ & $72.6 \%$ & $24.2 \%$ & $1.9 \%$ & $1.4 \%$ & $100.0 \%$ \\
\hline & \multirow{2}{*}{ Total } & Count & 156 & 55 & 15 & 6 & 232 \\
\hline & & $\%$ & $67.2 \%$ & $23.7 \%$ & $6.5 \%$ & $2.6 \%$ & $100.0 \%$ \\
\hline \multirow{4}{*}{4} & \multirow{2}{*}{$\begin{array}{c}\text { Shipment } \\
\text { to CL }\end{array}$} & Count & 3 & 3 & 11 & 2 & 19 \\
\hline & & $\%$ & $15.8 \%$ & $15.8 \%$ & $57.9 \%$ & $10.5 \%$ & $100.0 \%$ \\
\hline & \multirow{2}{*}{ Total } & Count & 3 & 3 & 11 & 2 & 19 \\
\hline & & $\%$ & $15.8 \%$ & $15.8 \%$ & $57.9 \%$ & $10.5 \%$ & $100.0 \%$ \\
\hline \multirow{4}{*}{5} & \multirow{2}{*}{ Spartan } & Count & 6 & 9 & 3 & 2 & 20 \\
\hline & & $\%$ & $30.0 \%$ & $45.0 \%$ & $15.0 \%$ & $10.0 \%$ & $100.0 \%$ \\
\hline & \multirow{2}{*}{ Total } & Count & 6 & 9 & 3 & 2 & 20 \\
\hline & & $\%$ & $30.0 \%$ & $45.0 \%$ & $15.0 \%$ & $10.0 \%$ & $100.0 \%$ \\
\hline \multirow{3}{*}{6} & Shipment & Count & 0 & & 3 & 4 & 7 \\
\hline & to $\mathrm{CL}$ & $\%$ & $0.0 \%$ & & $42.9 \%$ & $57.1 \%$ & $100.0 \%$ \\
\hline & Spartan & Count & 8 & & 1 & 0 & 9 \\
\hline
\end{tabular}

\begin{tabular}{|c|c|c|c|c|c|c|c|}
\hline & & $\%$ & $88.9 \%$ & & $11.1 \%$ & $0.0 \%$ & $100.0 \%$ \\
\hline & \multirow{2}{*}{ Total } & Count & 8 & & 4 & 4 & 16 \\
\hline & & $\%$ & $50.0 \%$ & & $25.0 \%$ & $25.0 \%$ & $100.0 \%$ \\
\hline \multirow{4}{*}{7} & \multirow{2}{*}{ Spartan } & Count & 26 & & & & 26 \\
\hline & & $\%$ & $100.0 \%$ & & & & $100.0 \%$ \\
\hline & \multirow{2}{*}{ Total } & Count & 26 & & & & 26 \\
\hline & & $\%$ & $100.0 \%$ & & & & $100.0 \%$ \\
\hline \multirow{6}{*}{8} & \multirow{2}{*}{$\begin{array}{c}\text { Shipment } \\
\text { to } \mathrm{CL}\end{array}$} & Count & 0 & 5 & 5 & 1 & 11 \\
\hline & & $\%$ & $0.0 \%$ & $45.5 \%$ & $45.5 \%$ & $9.1 \%$ & $100.0 \%$ \\
\hline & \multirow{2}{*}{ Spartan } & Count & 10 & 2 & 0 & 0 & 12 \\
\hline & & $\%$ & $83.3 \%$ & $16.7 \%$ & $0.0 \%$ & $0.0 \%$ & $100.0 \%$ \\
\hline & \multirow{2}{*}{ Total } & Count & 10 & 7 & 5 & 1 & 23 \\
\hline & & $\%$ & $43.5 \%$ & $30.4 \%$ & $21.7 \%$ & $4.3 \%$ & $100.0 \%$ \\
\hline \multirow{6}{*}{9} & \multirow{2}{*}{$\begin{array}{c}\text { Shipment } \\
\text { to } \mathrm{CL}\end{array}$} & Count & 0 & 0 & 15 & 64 & 79 \\
\hline & & $\%$ & $0.0 \%$ & $0.0 \%$ & $19.0 \%$ & $81.0 \%$ & $100.0 \%$ \\
\hline & \multirow{2}{*}{ Spartan } & Count & 12 & 21 & 9 & 5 & 47 \\
\hline & & $\%$ & $25.5 \%$ & $44.7 \%$ & $19.1 \%$ & $10.6 \%$ & $100.0 \%$ \\
\hline & \multirow{2}{*}{ Total } & Count & 12 & 21 & 24 & 69 & 126 \\
\hline & & $\%$ & $9.5 \%$ & $16.7 \%$ & $19.0 \%$ & $54.8 \%$ & $100.0 \%$ \\
\hline \multirow{8}{*}{ Total } & \multirow{2}{*}{$\begin{array}{l}\text { On-site } \\
\text { TaqMan }\end{array}$} & Count & 366 & 110 & 10 & 1 & 487 \\
\hline & & $\%$ & $75.2 \%$ & $22.6 \%$ & $2.1 \%$ & $0.2 \%$ & $100.0 \%$ \\
\hline & \multirow{2}{*}{$\begin{array}{c}\text { Shipment } \\
\text { to } \mathrm{CL}\end{array}$} & Count & 3 & 15 & 48 & 74 & 140 \\
\hline & & $\%$ & $2.1 \%$ & $10.7 \%$ & $34.3 \%$ & $52.9 \%$ & $100.0 \%$ \\
\hline & \multirow{2}{*}{ Spartan } & Count & 294 & 90 & 17 & 10 & 411 \\
\hline & & $\%$ & $71.5 \%$ & $21.9 \%$ & $4.1 \%$ & $2.4 \%$ & $100.0 \%$ \\
\hline & \multirow{2}{*}{ Total } & Count & 663 & 215 & 75 & 85 & 1038 \\
\hline & & $\%$ & $63.9 \%$ & $20.7 \%$ & $7.2 \%$ & $8.2 \%$ & $100.0 \%$ \\
\hline
\end{tabular}

Abbreviations: $\mathrm{CL}=$ central laboratory 


\section{A genotype-guided strategy for oral $P 2 Y_{12}$ inhibitors in primary $\mathrm{PCI}$}

Daniel M.F. Claassens, Gerrit J.A. Vos, Thomas O.Bergmeijer, Renicus S. Hermanides, Arnoud W.J. van 't Hof, Pim van der Harst,

Emanuele Barbato, Carmine Morisco, Richard M. Tjon Joe Gin, Folkert

W. Asselbergs, Arend Mosterd, Jean-PaulR. Herrman, Willem J.M.

Dewilde, Paul W.A. Janssen, Johannes C. Kelder, Maarten J. Postma,

Anthonius de Boer, Cornelis Boersma, Vera H.M. Deneer, Jurriën M. ten Berg

New England Journal of Medicine 2019; 381:1621-1631

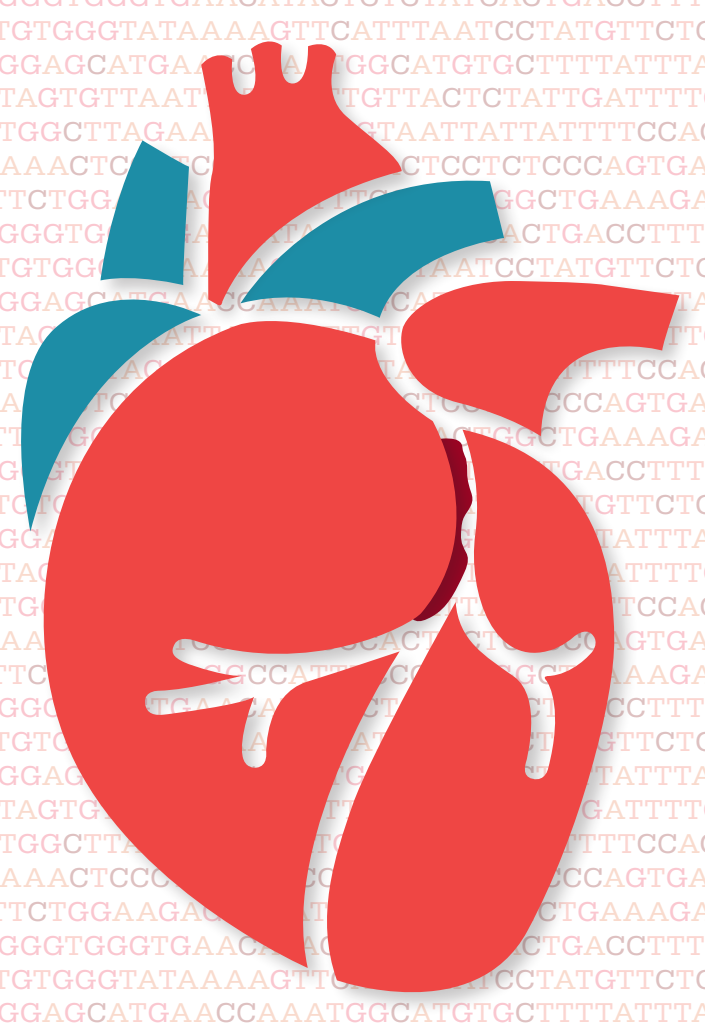




\section{Abstract}

\section{Background}

It is unknown whether patients undergoing primary percutaneous coronary intervention (PCI) benefit from genotype-guided selection of oral $\mathrm{P}_{2} \mathrm{Y}_{12}$ inhibitors.

\section{Methods}

We conducted a randomized, open-label, assessor-blinded trial in which patients undergoing primary PCI with stent implantation were assigned in a 1:1 ratio to receive either a $\mathrm{P}_{2} \mathrm{Y}_{12}$ inhibitor on the basis of early CYP2C19 genetic testing (genotype-guided group) or standard treatment with either ticagrelor or prasugrel (standard-treatment group) for 12 months. In the genotype-guided group, carriers of $C Y P 2 C 19 * 2$ or $C Y P 2 C 19 * 3$ loss-offunction alleles received ticagrelor or prasugrel, and noncarriers received clopidogrel. The two primary outcomes were net adverse clinical events defined as death from any cause, myocardial infarction, definite stent thrombosis, stroke, or major bleeding defined according to Platelet Inhibition and Patient Outcomes (PLATO) criteria - at 12 months (primary combined outcome; tested for noninferiority, with a noninferiority margin of 2 percentage points for the absolute difference) and PLATO major or minor bleeding at 12 months (primary bleeding outcome).

\section{Results}

For the primary analysis, 2488 patients were included: 1242 in the genotype-guided group and 1246 in the standard-treatment group. The primary combined outcome occurred in 63 patients $(5.1 \%)$ in the genotypeguided group and in 73 patients $(5.9 \%)$ in the standard-treatment group (absolute difference, -0.7 percentage points; $95 \%$ confidence interval $[\mathrm{CI}]$, -2.0 to $0.7 ; \mathrm{P}<0.001$ for noninferiority). The primary bleeding outcome occurred in 122 patients $(9.8 \%)$ in the genotype-guided group and in 156 patients $(12.5 \%)$ in the standard-treatment group (hazard ratio, $0.78 ; 95 \%$ $\mathrm{CI}, 0.61$ to $0.98 ; \mathrm{P}=0.04$ )

\section{Conclusion}

In patients undergoing primary PCI, a CYP2C19 genotype-guided strategy for selection of oral $\mathrm{P} 2 \mathrm{Y}_{12}$ inhibitor therapy was noninferior to standard treatment with ticagrelor or prasugrel at 12 months with respect to thrombotic events and resulted in a lower incidence of bleeding.

\section{Introduction}

In patients with ST-segment elevation myocardial infarction (STEMI), the preferred method of reperfusion is primary percutaneous coronary intervention (PCI) with stent implantation. In these patients, dual antiplatelet therapy, consisting of aspirin and a $\mathrm{P} 2 \mathrm{Y}_{12}$ inhibitor, is essential to prevent recurrent thrombotic events such as stent thrombosis [1] Current guidelines [1,2] favour the more potent platelet inhibitors ticagrelor and prasugrel over clopidogrel because these drugs are more effective for the prevention of thrombotic events [3,4]. However, this greater efficacy comes with a higher risk of bleeding $[3,4]$.

Clopidogrel is a prodrug, transformed into its active metabolite by hepatic cytochrome P450 enzymes. The active metabolite irreversibly inhibits the $\mathrm{P}_{2} \mathrm{Y}_{12}$ receptor on platelets, which results in inhibition of platelet aggregation. However, approximately $30 \%$ of white patients have an inadequate response to clopidogrel as measured with platelet-function tests [5]. Part of this variation in response can be explained by genetic variations, such as the $C Y P 2 C 19 * 2$ and $C Y P 2 C 19 * 3$ loss-of-function alleles [6-9]. In patients without these loss-of-function alleles, clopidogre has shown similar efficacy to that of ticagrelor and prasugrel [10-12]. We therefore conducted the CYP2C19 Genotype-Guided Antiplatelet Therapy in ST-Segment Elevation Myocardial Infarction Patients - Patient Outcome after Primary PCI (POPular Genetics) trial to determine whether a CYP2C19 genotype-guided strategy for selection of oral $\mathrm{P}_{2} \mathrm{Y}_{12}$ inhibitors can reduce bleeding risk without increasing thrombotic risk in patients with STEMI undergoing primary PCI with stent implantation.

\section{Methods}

Trial design

The POPular Genetics trial was an investigator-initiated, randomized, open-label, assessor-blinded trial performed at 10 European sites ( 8 in the Netherlands, 1 in Belgium, and 1 in Italy). It was sponsored by the Netherlands Organization for Health Research and Development, and Spartan Bioscience provided the Spartan RX point-of-care system and the reagents for free. Neither entity had any role in the design or execution of the trial or in the analysis of the data. Details of the design have been published previously [13].

The trial was approved by the appropriate ethics committees and national authorities in each country. The trial was registered after the enrolment of the first 18 patients, as described in the Supplementary Appendix 
(available with the full text of this article at NEJM.org). An independent data and safety monitoring board monitored the trial and had full access to all data. An independent clinical-event committee whose members were unaware of the trial-group assignments adjudicated all outcomes.

Trial monitoring was executed by an external service provider (Research Drive, Norg, the Netherlands). The authors vouch for the accuracy and completeness of the data and for the fidelity of the trial to the protocol, available at NEJM.org.

\section{Major protocol revision}

The initial plan was for the trial to compare a genotype-guided strategy for selecting oral $\mathrm{P}_{2} \mathrm{Y}_{12}$ inhibitors with a standard-treatment strategy for which clopidogrel was recommended. However, the 2011 European Society of Cardiology (ESC) guideline for patients with acute coronary syndrome without ST-segment elevation recommended use of ticagrelor or prasugre over clopidogrel.14 In anticipation that the same recommendation would be made for patients with ST-segment elevation, we changed the treatment in the standard-treatment group from clopidogrel to ticagrelor or prasugrel. Because we were now testing guided de-escalation of therapy instead of guided escalation of therapy, there were major changes in the primary outcomes and hypotheses of the trial. These changes are summarized in the Supplementary Appendix under "Major Protocol Revision May 2012."

\section{Trial population}

Patients were eligible for enrolment if they had signs or symptoms of STEMI lasting 30 minutes to 12 hours, underwent primary PCI with stent implantation, and were 21 years of age or older. Exclusion criteria are provided in Table S1 in the Supplementary Appendix. Because patients were asked to participate in the trial during the acute phase of myocardial infarction, many provided only oral informed consent before randomization. Such patients were eligible for the final analysis only if written informed consent was subsequently obtained.

\section{Pre-trial treatment}

Pre-trial antithrombotic treatment was administered according to local protocol. This usually consisted of a loading dose of aspirin, a $\mathrm{P} 2 \mathrm{Y}_{12}$ inhibitor, and heparin. Other antithrombotic periprocedural therapies were chosen at the discretion of the treating physician, as was the $\mathrm{P}_{2} \mathrm{Y}_{12}$ inhibitor administered before randomization.

\section{Randomization and trial procedures}

Using an Internet-based randomization procedure with computergenerated block randomization for each site, we randomly assigned patients in a 1:1 ratio to a CYP2C19 genotype-guided strategy or to standard treatment with ticagrelor or prasugrel (Fig. S1 in the Supplementary Appendix). Patients were enrolled during or up to 48 hours after primary PCI.

In patients assigned to the genotype-guided strategy, CYP2C19 genotyping was performed with the use of the TaqMan StepOnePlus assay at a centra laboratory (St. Antonius Hospital, Nieuwegein, the Netherlands) or with an on-site point-of-care Spartan RX device (Spartan Bioscience). Use of the Spartan device began during the course of the trial; not all hospitals started using it simultaneously. Genetic testing for the CYP $2 C 19 * 2$ and CYP2C19*3 loss-of-function alleles was performed as soon as possible after randomization. Details of the logistics of these tests in this trial have been published previously [15]. Carriers of a loss-of-function CYP2C19 allele were treated with ticagrelor or prasugrel, whereas noncarriers $\left(C Y P 2 C 19^{*} 1 / * 1\right)$ received clopidogrel according to the label instructions. If patients switched between $\mathrm{P} 2 \mathrm{Y}_{12}$ inhibitors, the use of a loading dose was at the discretion of the treating physician. Either ticagrelor or prasugre was prescribed to each patient in the standard-treatment group, according to local protocol.

All patients were treated with a $\mathrm{P}_{2} \mathrm{Y}_{12}$ inhibitor for at least 1 year after primary PCI. Patients were asked to fill out a questionnaire at 1 month, 6 months, and 12 months after primary PCI. Follow-up data were collected with the use of the hospital electronic health record. Questionnaires were used as an additional source of information. If necessary, the general practitioner, the patient's pharmacist, or the patient was contacted.

\section{Outcomes}

There were two primary outcomes. The first was the combined outcome of net adverse clinical events, which included death from any cause, myocardial infarction (defined according to the Third Universal Definition of Myocardial Infarction) [16], definite stent thrombosis (defined according to the Academic Research Consortium) [17], stroke (defined as a new neurologic deficit ending in death or lasting $>24$ hours, not due to another readily identifiable cause such as trauma), or major bleeding defined according to Platelet Inhibition and Patient Outcomes (PLATO) criteria (including major bleeding related to coronary-artery bypass grafting (CABG) as well as non-CABG-related major bleeding [18]), at 12 months (primary combined outcome). The second was PLATO major 
bleeding (CABG-related and non-CABG-related) or minor bleeding at 12 months (primary bleeding outcome). A complete list of secondary thrombotic and bleeding outcomes is provided in Tables S2 and S3 in the Supplementary Appendix.

\section{Statistical analysis}

The incidence of the primary combined outcome was assumed to be $18.8 \%$ in the standard-treatment group and $16.9 \%$ in the genotype-guided group These estimates were based on unpublished data from the Central Holland Acute Myocardial Infarction (CHAIR) study, the Ongoing Tirofiban in Myocardial Infarction Evaluation (On-TIME) 2 trial, and sub-analyses of the PLATO trial and the Trial to Assess Improvement in Therapeutic Outcomes by Optimizing Platelet Inhibition with Prasugrel-Thrombolysis in Myocardial Infarction (TRITON-TIMI) 38 [10,11,19]. Using a power of $80 \%$ and an alpha level of 0.05 and setting the noninferiority threshold for the absolute difference between the two groups in the incidence of the primary combined outcome at 2 percentage points, we calculated that 2474 patients would be needed to show noninferiority. For the primary bleeding outcome, the incidence was assumed to be $14.5 \%$ in the genotype-guided group and $18.9 \%$ in the standard-treatment group. Fewer patients were needed to show superiority for this outcome. Accounting for withdrawals and for approximately 200 patients enrolled before the May 2012 protocol amendment, we planned to include 2700 patients.

The end of the primary PCI procedure was defined as time 0 , irrespective of when randomization took place. Owing to the logistics of obtaining informed consent, obtaining genotyping results, and (if necessary) switching the $\mathrm{P} 2 \mathrm{Y}_{12}$ inhibitor, outcome events during the first 24 hours after primary PCI were excluded from the primary analysis. The primary analysis followed the intention-to-treat principle and included all patients enrolled after the May 2012 protocol amendment. In addition, a perprotocol analysis was performed. The primary combined outcome was first assessed in an analysis of the noninferiority of the CYP2C19 genotypeguided strategy to standard treatment. If noninferiority was confirmed, superiority was assessed. For the primary bleeding outcome, the CYP2C19 genotype-guided strategy was assumed to be superior to standard treatment. No adjustment for alpha error was performed to account for the assessment of two primary outcomes, because both analyses were required to show significance at a $\mathrm{P}$ value of less than 0.05 in order to assert a beneficial effect of the genotype-guided strategy. Both primary outcomes were also assessed in 26 prespecified subgroups. Two

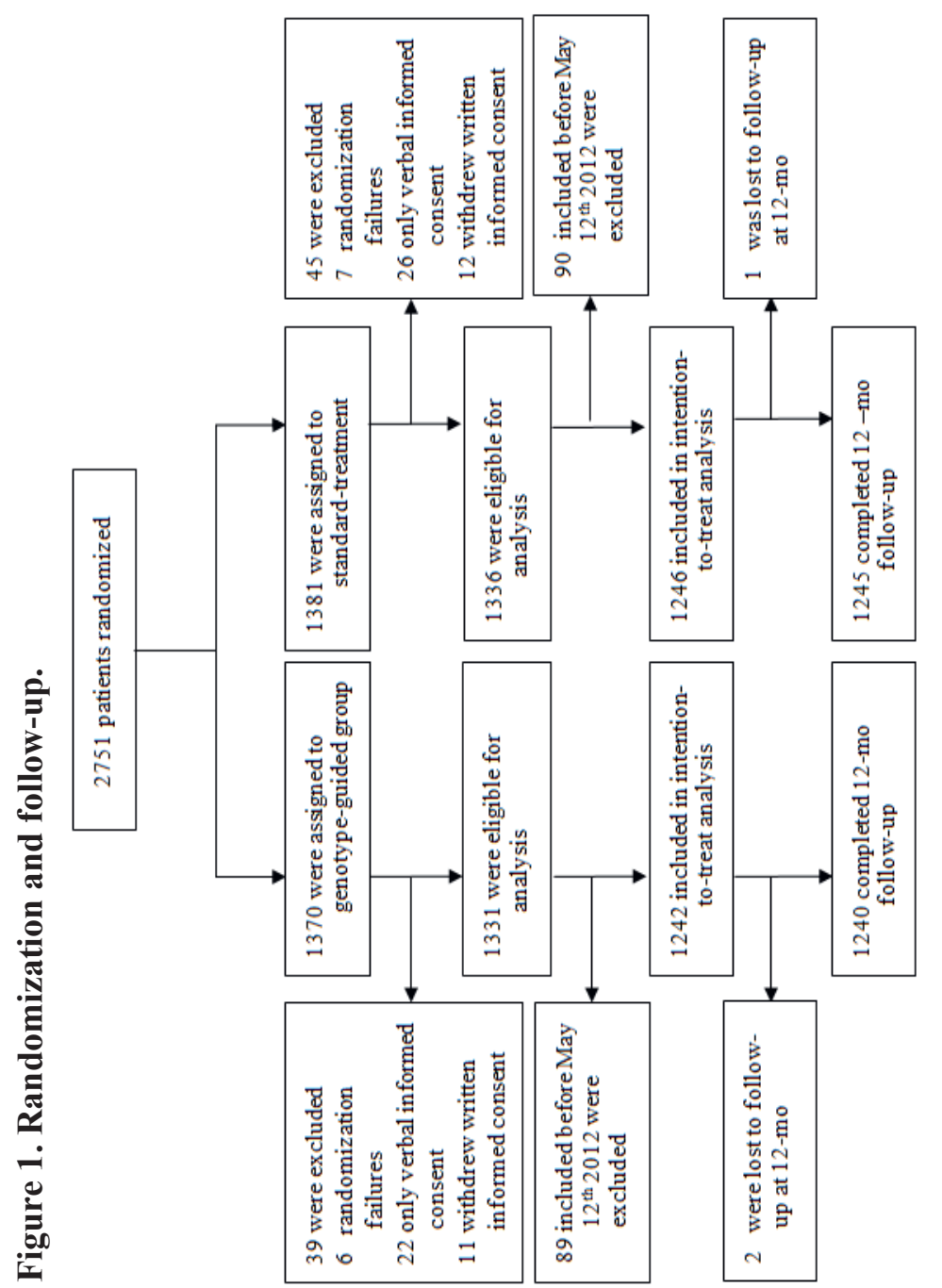


sensitivity analyses were planned. The first included events during the 24 hours after primary PCI, and the second analysis involved stratification according to centre.

Time-to-event curves were constructed with the use of the Kaplan-Meier method. Differences between the survival curves were compared with the use of the log-rank test, and Cox proportional-hazards models were used to calculate hazard ratios with $95 \%$ confidence intervals. Confidence intervals for secondary outcomes have not been adjusted for multiple comparisons, and therefore inferences drawn from these intervals may not be reproducible. $P$ values were two-sided, and values of less than 0.05 were considered to indicate statistical significance. Data were analysed with $\mathrm{R}$ software, version 3.6.0 (R Foundation for Statistical Computing).

\section{Results}

\section{Participants and follow-up}

From June 2011 through April 2018, a total of 2751 patients were randomly assigned to a treatment group (Fig. 1). A screening log was kep at only one centre; the records of those patients screened are shown in Table S4 in the Supplementary Appendix. Patients who underwen duplicate randomization in error, those who withdrew consent, and those who were enrolled before the protocol amendment of May 2012 were excluded from all analyses. Therefore, 2488 patients entered the intentionto-treat analysis (1242 in the genotype-guided group and 1246 in the standard-treatment group), of whom 3 were lost to follow-up. The database was locked on May 28, 2019, after all trial participants had completed at least 12 months of follow-up.

Table 1. (see next page) Baseline Characteristics of the Patients and Initia Antithrombotic Therapy.* Plus-minus values are means \pm SD. PCI denotes percutaneous coronary intervention. $\dagger$ The body-mass index is the weight in kilograms divided by the square of the height in meters. Data were missing for 66 patients in the genotype-guided group and 77 in the standard-treatment group. The creatinine clearance was calculated with the use of the Chronic Kidney Disease Epidemiology Collaboration formula.
Table 1. Baseline characteristics of the patients and initia antithrombotic therapy.*

\begin{tabular}{|c|c|c|}
\hline Characteristic & $\begin{array}{c}\begin{array}{c}\text { Genotype- } \\
\text { guided group }\end{array} \\
(\mathrm{N}=\mathbf{1 2 4 2})\end{array}$ & $\begin{array}{c}\text { Standard- } \\
\text { treatment } \\
\text { group } \\
(\mathbf{N}=\mathbf{1 2 4 6})\end{array}$ \\
\hline \multicolumn{3}{|l|}{ Baseline characteristics } \\
\hline Age - yr & $61.9 \pm 11.1$ & $61.4 \pm 11.5$ \\
\hline Age $\geq 75$ yr - no. $(\%)$ & $188(15.1)$ & $175(14.0)$ \\
\hline Female sex - no. $(\%)$ & $317(25.5)$ & $309(24.8)$ \\
\hline Body-mass index $\dagger$ & $27.5 \pm 6.67$ & $27.0 \pm 4.27$ \\
\hline $\begin{array}{l}\text { Creatinine clearance }<60 \\
\mathrm{ml} / \mathrm{min} / 1.73 \mathrm{~m} 2 \text { at baseline }- \text { no. }(\%) \\
\$\end{array}$ & $121(9.8)$ & $109(8.8)$ \\
\hline \multicolumn{3}{|l|}{ Cardiovascular risk factors } \\
\hline Current smoker - no. (\%) & $562(45.8)$ & $565(45.8)$ \\
\hline Diabetes mellitus - no. (\%) & $150(12.1)$ & $138(11.1)$ \\
\hline Arterial hypertension - no. (\%) & $521(41.9)$ & $511(41.0)$ \\
\hline Hyperlipidaemia — no. & $260 /(21.0)$ & $255(20.5)$ \\
\hline $\begin{array}{l}\text { Family history of coronary artery } \\
\text { disease - no./total no. }(\%)\end{array}$ & 475 (40.6) & $467(39.5)$ \\
\hline \multicolumn{3}{|l|}{ Other medical history — no. (\%) } \\
\hline Previous PCI with stenting & $99(8.0)$ & $91(7.3)$ \\
\hline $\begin{array}{l}\text { Previous coronary-artery bypass } \\
\text { grafting }\end{array}$ & $12(1.0)$ & $22(1.8)$ \\
\hline Previous myocardial infarction & $97(7.8)$ & $87(7.0)$ \\
\hline History of coronary artery disease & $133(10.7)$ & $118(9.5)$ \\
\hline Peripheral arterial disease & $39(3.1)$ & $34(2.7)$ \\
\hline History of bleeding & $30(2.4)$ & $23(1.8)$ \\
\hline $\begin{array}{l}\text { Aspirin use before primary PCI - } \\
\text { no. }(\%)\end{array}$ & $1232(99.4)$ & $1238(99.4)$ \\
\hline $\begin{array}{l}\text { P2Y } 12 \text { inhibitor use before primary } \\
\text { PCI - no. (\%) }\end{array}$ & $1198(96.8)$ & $1190(96.0)$ \\
\hline \multicolumn{3}{|c|}{$\begin{array}{l}\text { Initial antithrombotic therapy after randomization and genotyping - } \\
\text { no./total no. (\%) }\end{array}$} \\
\hline \multicolumn{3}{|l|}{$\mathrm{P} 2 \mathrm{Y}_{12}$ inhibitor } \\
\hline Any & $1236(99.8)$ & $1237(99.8)$ \\
\hline Clopidogrel & $751(60.6)$ & $87(7.0)$ \\
\hline Prasugrel & $13(1.0)$ & $28(2.3)$ \\
\hline Ticagrelor & $472(38.1)$ & $1122(90.5)$ \\
\hline None & $3(0.2)$ & $3(0.2)$ \\
\hline Aspirin & $1211(97.7)$ & $1208(97.4)$ \\
\hline Oral anticoagulation & $51(4.1)$ & $54(4.4)$ \\
\hline
\end{tabular}


The two groups were balanced with regard to all baseline characteristics and non-trial-related medications and procedures (Table 1, and Tables S5 and S6 in the Supplementary Appendix). Table S7 in the Supplementary Appendix provides an overview of the genotype results, and Table S8 provides an overview of the intervals from symptom onset to the time that a patient was receiving the recommended therapy. In the genotype-guided group, $60.6 \%$ of the patients received clopidogrel and $39.1 \%$ received ticagrelor or prasugrel after their genetic results were available (Table 1). In the standard-treatment group, $92.7 \%$ of the patients were treated with ticagrelor or prasugrel and $7.0 \%$ with clopidogrel.

The percentage of patients who adhered to the recommended trial drug during the entire duration of follow-up, as assessed by the investigators, was $84.5 \%$ in the genotype-guided group and $82.0 \%$ in the standardtreatment group. Tables S9A, B, and C in the Supplementary Appendix provide an overview of how many patients switched or discontinued a $\mathrm{P} 2 \mathrm{Y}_{12}$ inhibitor and for what reasons, and Table S10 provides an overview of $\mathrm{P}_{2} \mathrm{Y}_{12}$ inhibitor use during follow-up.

\section{Primary outcomes}

At 12 months, death from any cause, myocardial infarction, definite sten thrombosis, stroke, or PLATO major bleeding (the primary combined outcome) occurred in 63 patients (5.1\%) in the genotype-guided group and in 73 patients $(5.9 \%)$ in the standard-treatment group. The genotypeguided strategy met the prespecified criterion for noninferiority with respect to net adverse clinical events (absolute difference in incidence, -0.7 percentage points; $95 \%$ confidence interval $[\mathrm{CI}],-2.0$ to $0.7 ; \mathrm{P}<0.001$ for noninferiority) (Fig. 2A and Table 2). The analysis for superiority did not support superiority of the genotype-guided strategy (hazard ratio, 0.87 $95 \% \mathrm{CI}, 0.62$ to $1.21 ; \mathrm{P}=0.40$ ). The primary bleeding outcome (PLATO major or minor bleeding) was significantly less common in the genotypeguided group than in the standard-treatment group (122 events [9.8\%] vs. 156 [12.5\%]; hazard ratio, $0.78 ; 95 \% \mathrm{CI}, 0.61$ to $0.98 ; \mathrm{P}=0.04$ ) (Fig. 2B and Table 3 ). The results of the per-protocol analysis and the sensitivity analyses were consistent with those of the primary analyses (Tables S11 and S12 in the Supplementary Appendix).

\section{Secondary outcomes}

There were no significant differences between the two groups for the combined thrombotic outcome consisting of death from vascular causes, myocardial infarction, definite stent thrombosis, or stroke $(2.7 \%$ in the genotype-guided group and $3.3 \%$ in the standard-treatment group; hazard ratio, $0.83 ; 95 \% \mathrm{CI}, 0.53$ to 1.31 ) or for any of the other secondary thrombotic outcomes (Table 2, and Fig. S2 in the Supplementary Appendix). Furthermore, there was no difference in the incidence of PLATO major bleeding between the genotype-guided group and the standard-treatment group (2.3\% in both groups; hazard ratio, $0.97 ; 95 \% \mathrm{CI}$ 0.58 to 1.63$)$. The between-group difference in the primary bleeding outcome was driven by a lower incidence of PLATO minor bleeding in the genotype-guided group (7.6\% vs. $10.5 \%$; hazard ratio, $0.72 ; 95 \% \mathrm{CI}, 0.55$ to 0.94 ) (Fig. S3 in the Supplementary Appendix). Table 3 shows a full list of bleeding outcomes.

Analyses of the primary outcomes were performed in prespecified subgroups. The results were generally consistent with those in the whole cohort (Figs. S4 and S5 in the Supplementary Appendix).

\section{Discussion}

In this trial, we investigated the possible clinical benefit of CYP2C19 genotype-guided antiplatelet therapy in patients with STEMI undergoing primary PCI. There are two key findings from this trial. First, the use of a genotype-guided strategy, in which patients without a CYP2C19 loss-offunction allele received clopidogrel, was not associated with a higher risk of combined death from any cause, myocardial infarction, definite stent thrombosis, stroke, or major bleeding 12 months after primary PCI than standard treatment with the more potent $\mathrm{P}_{2} \mathrm{Y}_{12}$ inhibitors ticagrelor and prasugrel. Second, the use of clopidogrel in the genotype-guided group resulted in a lower risk of (mostly minor) bleeding than standard treatment.

Since the trial was designed, the incidence of thrombotic events after acute coronary syndromes has decreased considerably, particularly because of the use of newer-generation stents [20]. Other recent trials with similar populations, such as the Dual Antiplatelet Therapy after Drug-Eluting Stent Implantation in ST-Elevation Myocardial Infarction (DAPT-STEMI) trial and the Testing Responsiveness to Platelet Inhibition on Chronic Antiplatelet Treatment for Acute Coronary Syndromes (TROPICAL-ACS) trial, have shown a similar incidence of thrombotic events [21,22]. With the lower-than-anticipated incidence of the primary combined outcome in our trial, the prespecified noninferiority margin was wider relative to the incidence than originally expected. The upper boundary of the $95 \%$ confidence interval for the primary combined outcome was $21 \%$ higher than the incidence in the standard-treatment group. However, the observed upper boundary of the $95 \%$ confidence interval in the per-protocol analysis (11\% higher than the incidence in the standard-treatment group) (Table S11 in the Supplementary Appendix) gives stronger support to the 

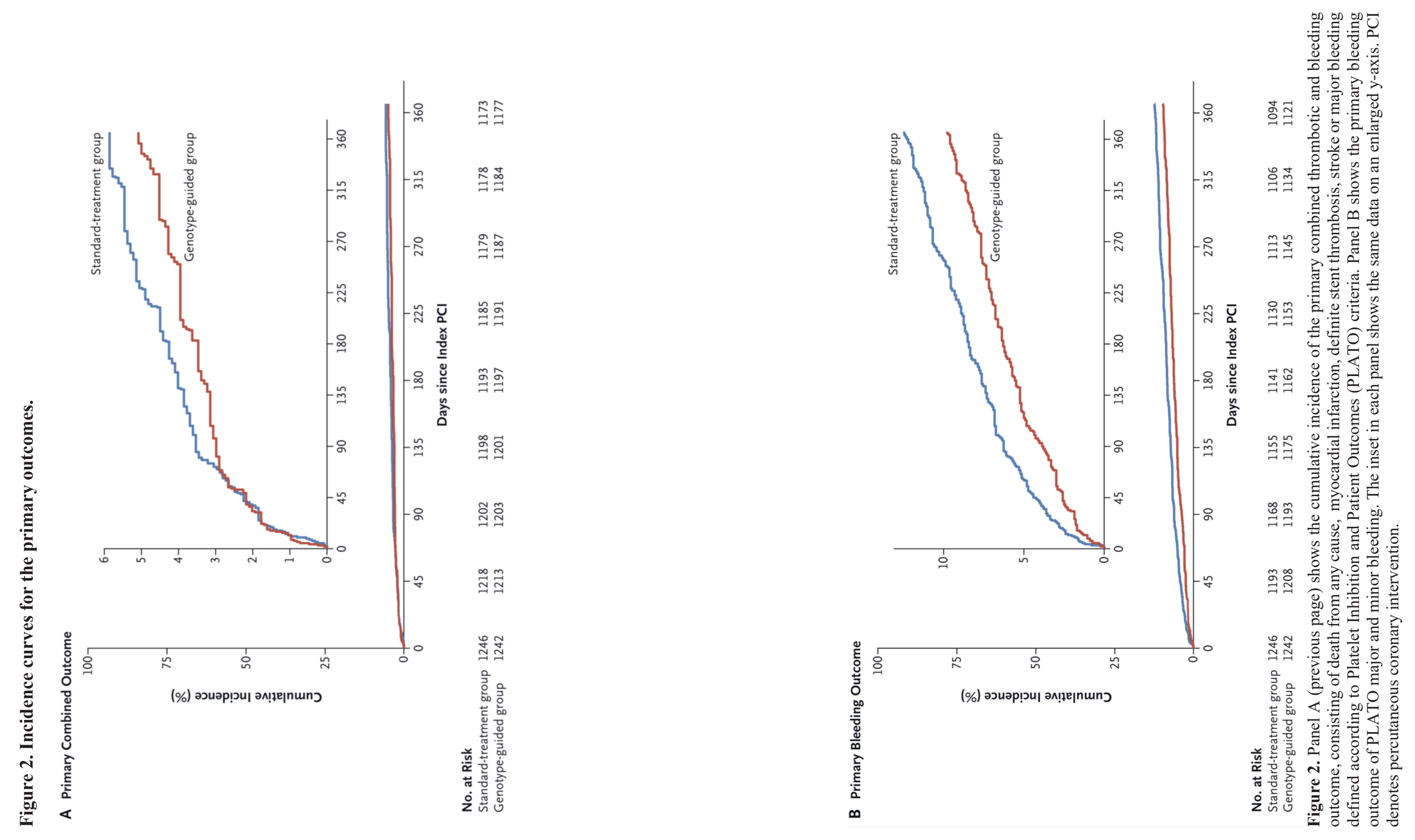

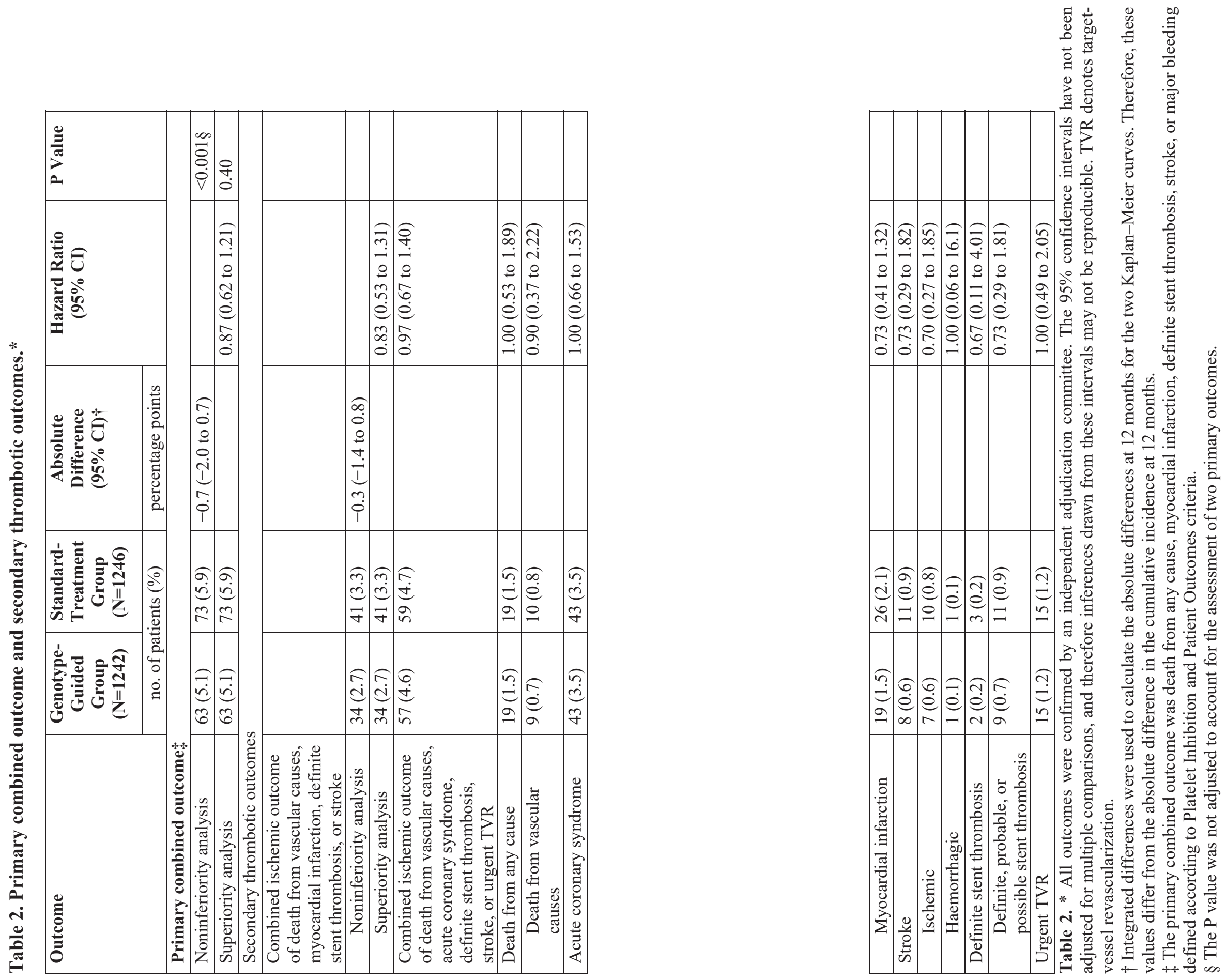

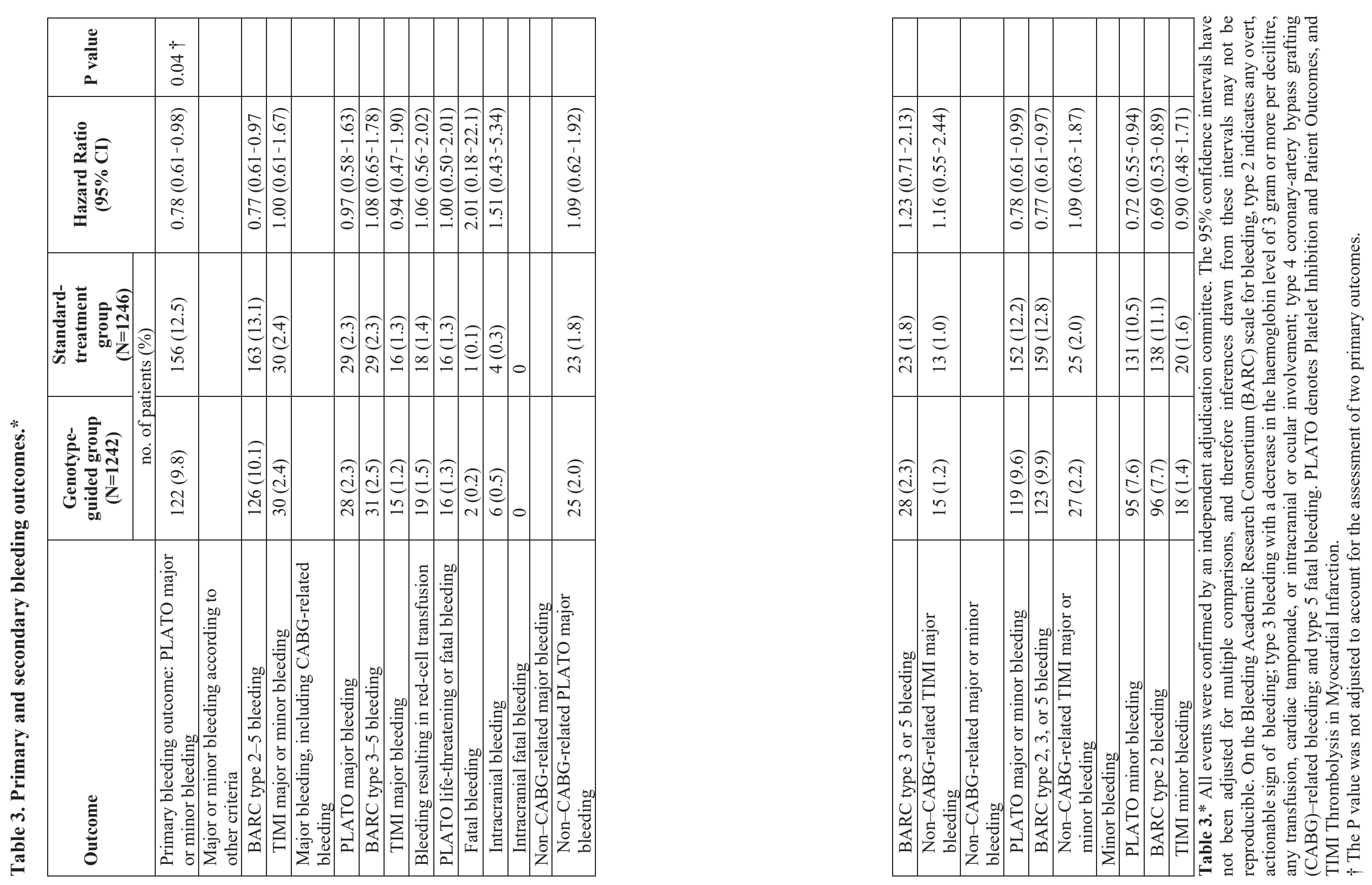
conclusion that genotype-guided $\mathrm{P} 2 \mathrm{Y}_{12}$ treatment is noninferior to standard treatment for the occurrence of thrombotic events. The results for both the thrombotic and bleeding outcomes of the trial are in line with data from observational studies and smaller trials investigating a genotype-guided strategy [13,23-26].

Guided de-escalation was also investigated in the TROPICAL-ACS trial, which included 2610 patients with biomarker-positive acute coronary syndromes undergoing PCI. It showed that guided de-escalation of $\mathrm{P}_{2} \mathrm{Y}_{12}$ inhibitor therapy (switching to clopidogrel) with the use of plateletfunction testing was noninferior to prasugrel for the prevention of thrombotic events [22]. However, the incidence of bleeding was not significantly lower in the de-escalation group than in the prasugrel group. Furthermore, this method of guiding therapy requires patients to switch between $\mathrm{P}_{2} \mathrm{Y}_{12}$ inhibitors multiple times if they have high platelet reactivity during treatment with clopidogrel, and it requires patients to revisit the clinic to perform platelet function testing. On the basis of the TROPICAL-ACS trial, the latest ESC guidelines give a class IIb recommendation to use platelet-function testing for guided de-escalation, especially in patients who are not deemed to be candidates for 12 months of potent antiplatelet therapy [27]

Trials such as TROPICAL-ACS usually included Bleeding Academic Research Consortium (BARC) type 2 bleeding (any overt, actionable sign of bleeding) in their definition of net clinical benefit $[22,28]$. We chose to incorporate only major bleeding, which has an effect on morbidity and mortality similar to that of thrombotic outcomes [29]. Furthermore, although earlier trials consistently showed a lower incidence of minor bleeding with clopidogrel than with prasugrel or ticagrelor, this effect was not seen for major bleeding $[3,4,22,28]$. Our data confirm this finding. Therefore, guided de-escalation will mostly help to reduce minor bleeding. Nevertheless, minor bleeding is of clinical relevance, since it leads to medical intervention and influences treatment adherence and health care cost [30-32].

Limitations of our trial include the open-label design, the lower-thananticipated incidence of the primary combined outcome, and the fact that genetic variation is not the only factor contributing to high platelet reactivity. Moreover, there are more polymorphisms of the CYP2C19 gene, although most are very rare [13], which may be associated with increased thrombotic or bleeding risk. However, data on these other alleles are conflicting [33]. Therefore, our strategy based solely on the CYP2C19 genotype may not be the most useful strategy for some patients.
In conclusion, in patients with STEMI undergoing primary PCI, a CYP2C19 genotype-guided strategy for selection of oral $\mathrm{P}_{2} \mathrm{Y}_{12}$ inhibitor therapy was noninferior to standard treatment with ticagrelor or prasugrel at 12 months with respect to thrombotic events and resulted in a lower incidence of bleeding. 


\section{References}

1. Ibanez B, James S, Agewall S, et al. 2017 ESC guidelines for the management of acute myocardial infarction in patients presenting with ST-segment elevation. The Task Force for the management of acute myocardial infarction in patients presenting with ST-segment elevation of the European Society of Cardiology (ESC). Eur Heart J 2018; 39:119177

2. Levine GN, Bates ER, Bittl JA, et al. 2016 ACC/AHA guideline focused update on duration of dual antiplatelet therapy in patients with coronary artery disease: a report of the American College of Cardiology/American Heart Association Task Force on Clinical Practice Guidelines. J Am Coll Cardiol 2016; 68:1082-1115

3. Wallentin L, Becker RC, Budaj A, et al. Ticagrelor versus clopidogrel in patients with acute coronary syndromes. N Engl J Med 2009; 361:104557

4. Wiviott SD, Braunwald E, McCabe $\mathrm{CH}$, et al. Prasugrel versus clopidogrel in patients with acute coronary syndromes. N Engl J Med 2007; 357:2001-2015

5. Breet NJ, van Werkum JW, Bouman HJ, et al. Comparison of platelet function tests in predicting clinical outcome in patients undergoing coronary stent implantation. JAMA 2010; 303:754-762

6. Mega JL, Simon T, Collet JP, et al. Reduced-function CYP2C19 genotype and risk of adverse clinical outcomes among patients treated with clopidogrel predominantly for PCI: a meta-analysis. JAMA 2010, 304:1821-1830

7. Collet JP, Hulot JS, Pena A, et al. Cytochrome P450 2C19 polymorphism in young patients treated with clopidogrel after myocardial infarction: a cohort study. Lancet 2009; 373:309-317

8. Harmsze AM, van Werkum JW, Ten Berg JM, et al. CYP2C19*2 and CYP2C9*3 alleles are associated with stent thrombosis: a case-control study. Eur Heart J 2010; 31:3046-3053

9. Shuldiner AR, O'Connell JR, Bliden KP, et al. Association of cytochrome P450 2C19 genotype with the antiplatelet effect and clinical efficacy of clopidogrel therapy. JAMA 2009; 302:849-857

10. Mega JL, Close SL, Wiviott SD, et al. Genetic variants in ABCB1 and CYP2C19 and cardiovascular outcomes after treatment with clopidogre and prasugrel in the TRITON-TIMI 38 trial: a pharmacogenetic analysis Lancet 2010; 376:1312-1319

11. Wallentin L, James S, Storey RF, et al. Effect of CYP2C19 and ABCB single nucleotide polymorphisms on outcomes of treatment with ticagrelor versus clopidogrel for acute coronary syndromes: a genetic substudy of the PLATO trial. Lancet 2010; 376:1320-1328

12. Cavallari LH, Lee CR, Beitelshees AL, et al. Multisite investigation of outcomes with implementation of CYP2C19 genotype-guided antiplatelet therapy after percutaneous coronary intervention. JACC Cardiovasc Interv 2018; 11:181-191

13. Bergmeijer TO, Janssen PW, Schipper JC, et al. CYP2C19 genotypeguided antiplatelet therapy in ST-segment elevation myocardia infarction patients - rationale and design of the Patient Outcome after primary PCI (POPular) Genetics study. Am Heart J 2014; 168:1622.e1

14. Hamm CW, Bassand JP, Agewall S, et al. ESC guidelines for the management of acute coronary syndromes in patients presenting without persistent ST-segment elevation: the task force for the management of acute coronary syndromes (ACS) in patients presenting without persistent ST-segment elevation of the European Society of Cardiology (ESC). Eur Heart J 2011; 32:2999-3054

15. Bergmeijer TO, Vos GJ, Claassens DM, et al. Feasibility and implementation of CYP2C19 genotyping in patients using antiplatelet therapy. Pharmacogenomics 2018; 19:621-628

16. Thygesen K, Alpert JS, Jaffe AS, et al. Third universal definition of myocardial infarction. J Am Coll Cardiol 2012; 60:1581-1598

17. Cutlip DE, Windecker S, Mehran R, et al. Clinical end points in coronary stent trials: a case for standardized definitions. Circulation 2007 115:2344-2351

18. Mehran R, Rao SV, Bhatt DL, et al. Standardized bleeding definitions for cardiovascular clinical trials: a consensus report from the Bleeding Academic Research Consortium. Circulation 2011; 123:2736-2747

19. Van't Hof AW, Ten Berg J, Heestermans T, et al. Prehospital initiation of tirofiban in patients with ST-elevation myocardial infarction undergoing primary angioplasty (On-TIME 2): a multicentre, doubleblind, randomised controlled trial. Lancet 2008; 372:537-546

20. Byrne RA, Joner M, Kastrati A. Stent thrombosis and restenosis: what have we learned and where are we going? The Andreas Grüntzig Lecture ESC 2014. Eur Heart J 2015; 36:3320-3331

21. Kedhi E, Fabris E, van der Ent M, et al. Six months versus 12 month dual antiplatelet therapy after drug-eluting stent implantation in STelevation myocardial infarction (DAPT-STEMI): randomised, multicentre, non-inferiority trial. BMJ 2018; 363:k3793

22. Sibbing D, Aradi D, Jacobshagen $C$, et al. Guided de-escalation of antiplatelet treatment in patients with acute coronary syndrome undergoing percutaneous coronary intervention (TROPICAL-ACS): a randomised, open-label, multicentre trial. Lancet 2017; 390:1747-1757

23. Sánchez-Ramos J, Dávila-Fajardo CL, Toledo Frías P, et al. Results of genotype-guided antiplatelet therapy in patients who undergone percutaneous coronary intervention with stent. Int J Cardiol 2016; 225:289-295

24. Roberts JD, Wells GA, Le May MR, et al. Point-of-care genetic testing for personalisation of antiplatelet treatment (RAPID GENE): - a 
prospective, randomised, proof-of-concept trial. Lancet 2012; 379:17051711

25. Janssen PWA, Bergmeijer TO, Vos GA, et al. Tailored P2Y12 inhibitor treatment in patients undergoing non-urgent PCI - the POPular Risk Score study. Eur J Clin Pharmacol 2019; 75:1201-1210

26. Notarangelo FM, Maglietta G, Bevilacqua P, et al. Pharmacogenomic approach to selecting antiplatelet therapy in patients with acute coronary syndromes: the PHARMCLO Trial. J Am Coll Cardiol 2018; 71:18691877

27. Neumann FJ, Sousa-Uva M, Ahlsson A, et al. 2018 ESC/EACTS guidelines on myocardial revascularization. Eur Heart J 2019; 40:87-165

28. Cayla G, Cuisset T, Silvain J, et al. Platelet function monitoring to adjust antiplatelet therapy in elderly patients stented for an acute coronary syndrome (ANTARCTIC): an open-label, blinded endpoint,

randomised controlled superiority trial. Lancet 2016; 388:2015-2022

29. Généreux P, Giustino G, Witzenbichler B, et al. Incidence, predictors, and impact of post-discharge bleeding after percutaneous coronary intervention. J Am Coll Cardiol 2015; 66:1036-1045

30. Czarny MJ, Nathan AS, Yeh RW, et al. Adherence to dual antiplatelet therapy after coronary stenting: a systematic review. Clin Cardiol 2014 37:505-513

31. Cutlip DE, Kereiakes DJ, Mauri L, et al. Thrombotic complications associated with early and late nonadherence to dual antiplatelet therapy. JACC Cardiovasc Interv 2015; 8:404-410

32. Straub N, Beivers A, Lenk E, et al. A model-based analysis of the clinical and economic impact of personalising P2Y12-receptor inhibition with platelet function testing in acute coronary syndrome patients. Thromb Haemost 2014; 111:290-299

33. Sibbing D, Aradi D, Alexopoulos D, et al. Updated expert consensus statement on platelet function and genetic testing for guiding P2Y12 receptor inhibitor treatment in percutaneous coronary intervention. JACC Cardiovasc Interv 2019; 12:1521-1537

\section{Supplementary appendix}




\section{Major protocol revision May 2012}

When the trial started in July 2011, clopidogrel was the recommended P2 $\mathrm{Y}_{12}$ inhibitor. Patients in the standard-treatment group therefore received clopidogrel and patients in the genotype-guided group received either clopidogrel, or, if they carried CYP $2 C 19 * 2$ or *3 loss-of-function alleles, they received more potent antithrombotic therapy with prasugrel (See Protocol Version 3, available online at NEJM.org). At the time, we hypothesised that a genotype-guided strategy would be superior to standard treatment with clopidogrel with regard to a primary outcome consisting of only thrombotic events (all-cause death, myocardial infarction, stent thrombosis, urgent target-vessel revascularization and stroke). We also wanted to assess the safety of this strategy in terms of non-CABG related major bleeding.

In August 2011, the European Society of Cardiology published a guideline for patients with acute coronary syndromes without ST-segment elevation, which was later published in the European Heart Journal in December 2011 [1]. In this guideline the use of ticagrelor or prasugrel was recommended over clopidogrel in these patients. We anticipated that these changes would also follow in the guideline for patients with ST-segment elevation, which was published a year later, in 2012. In anticipation of this change we amended the protocol.

In the amended protocol, dated 16 February 2012, the standard-treatment group received either ticagrelor or prasugrel, as recommended by the guidelines (See Protocol Version 5, available online at NEJM.org). No changes were made to the genotype-guided group. Enrolment of patients under the amended protocol began on May 12, 2012.

Because of this change, patients in the genotype-guided group who did not carry $C Y P 2 C 19 * 2$ or $* 3$ loss-of-function alleles now received less potent antithrombotic therapy than patients in the standard-treatment group. This led to changes in the hypotheses and the two primary outcomes. We now hypothesised that the genotype-guided group would be non-inferior for the primary outcome, which included both thrombotic outcomes and PLATO major bleeding. We did not expect a difference in non-CABG related major bleeding between these treatment arms anymore, but we did expect there to be an effect on all bleedings. Therefore, we hypothesised that the genotype-guided group would be superior in reducing combined PLATO
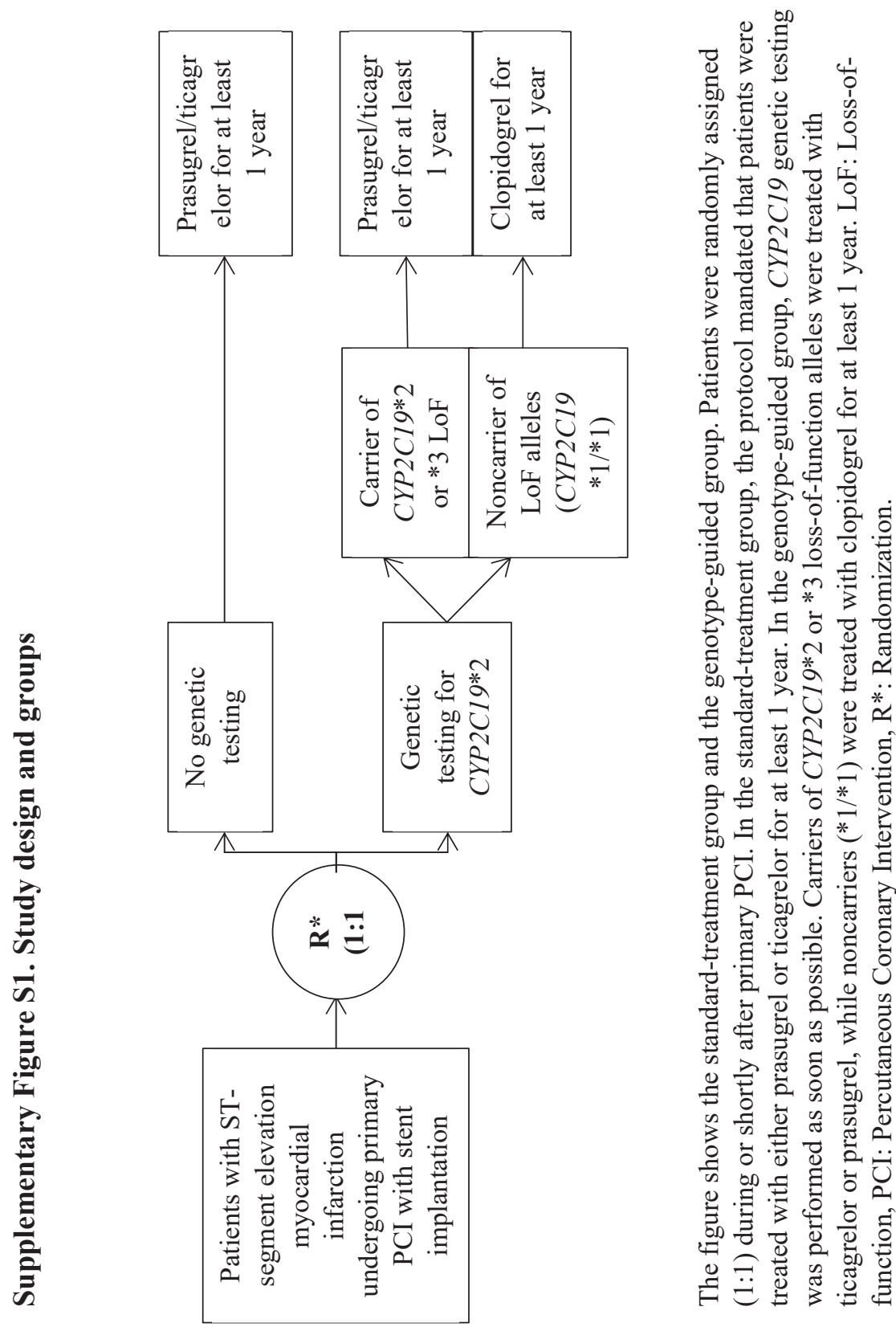

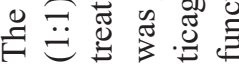



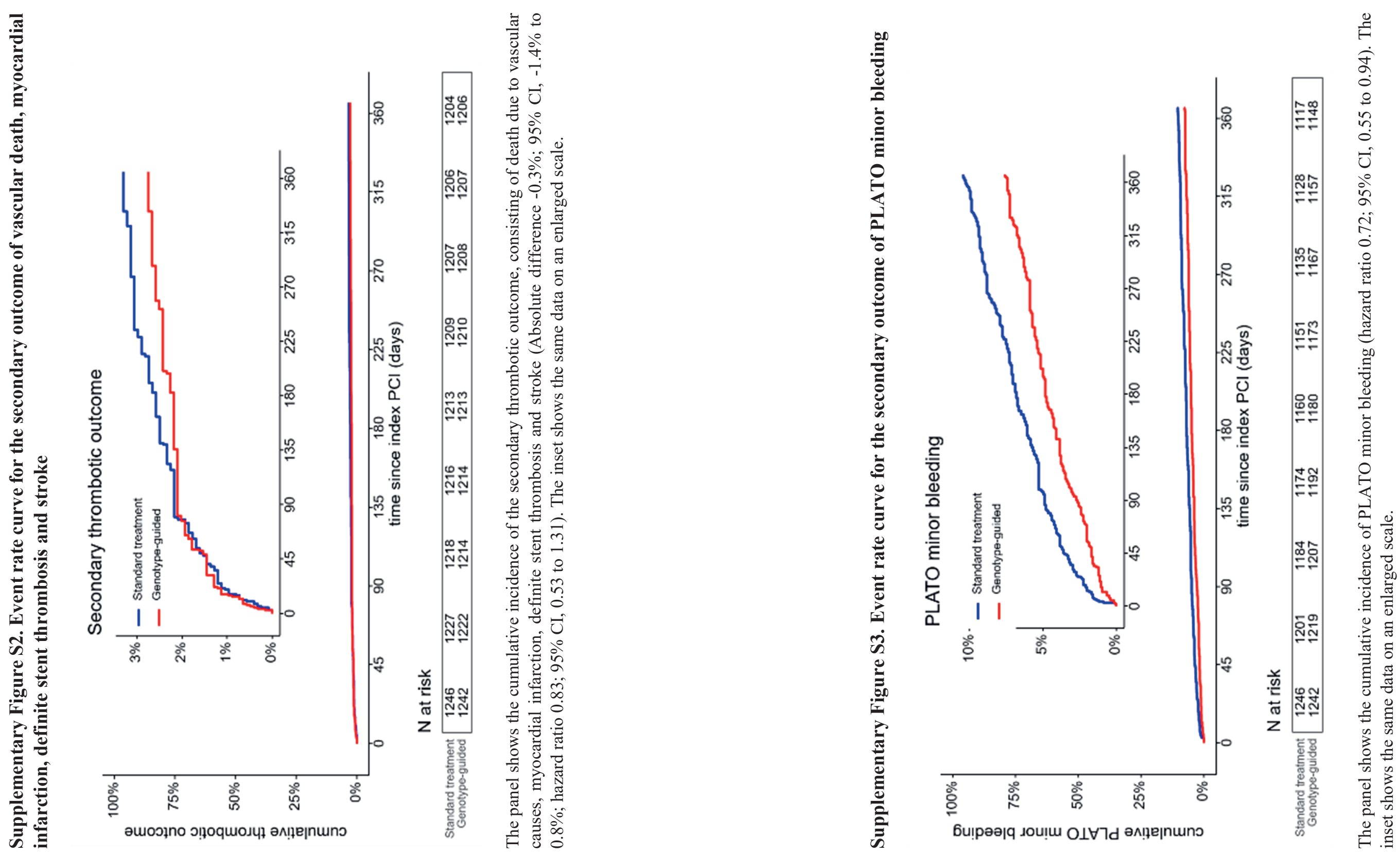
Supplementary Figure S4. Subgroup analyses for the combined primary thrombotic and bleeding outcome

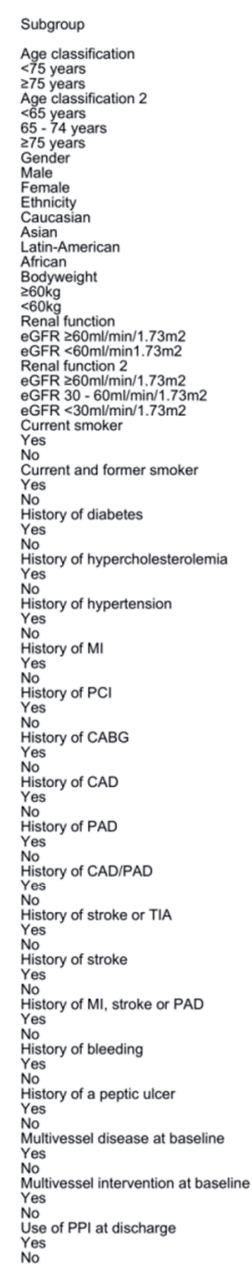

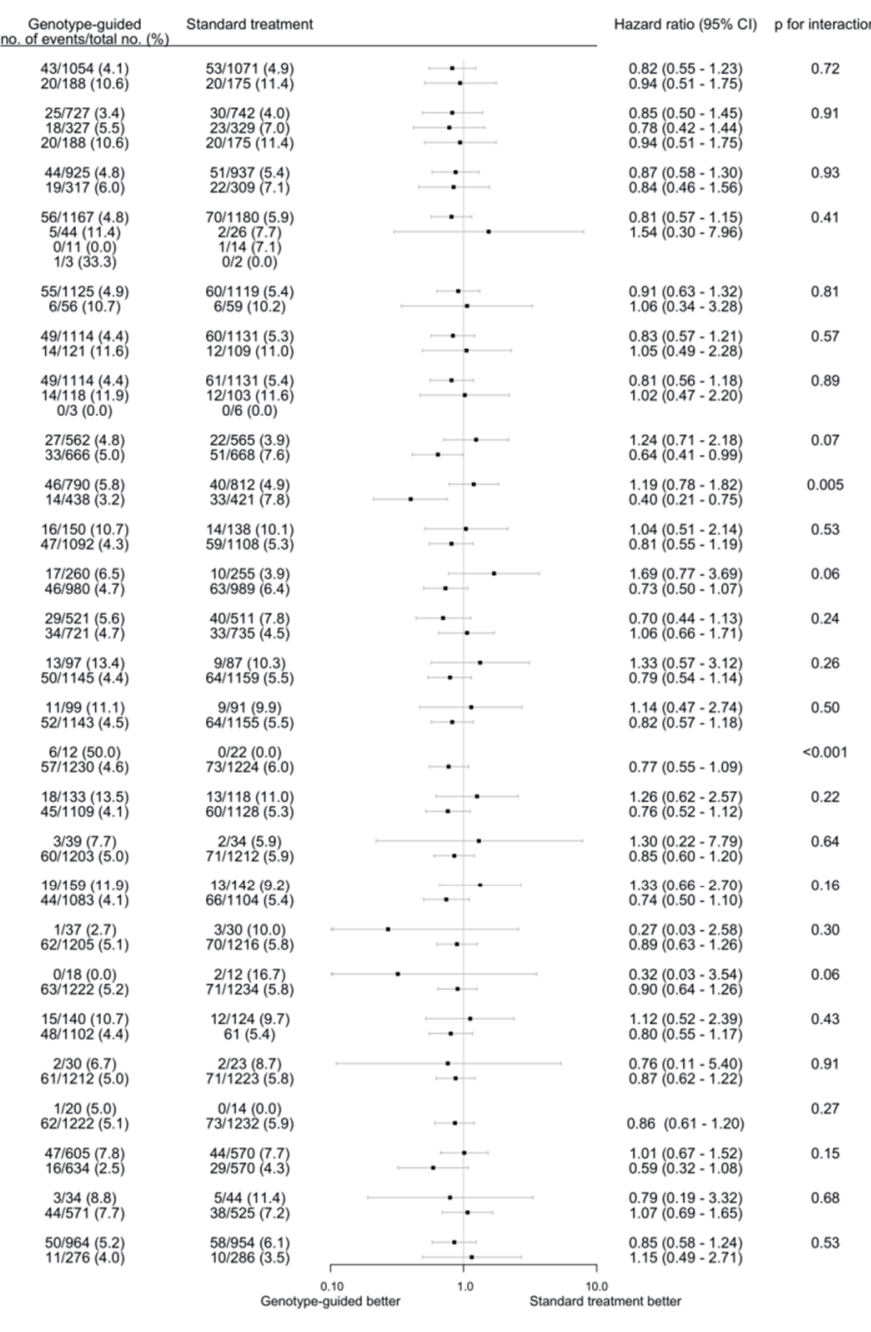

Analyses for 26 pre-specified subgroups for the primary combined thrombotic and bleeding outcome. eGFR: estimated glomerular filtration rate in $\mathrm{ml} / \mathrm{min} / 1.73 \mathrm{~m} 2$ calculated using the CKD-EPI formula; CAD: coronary artery disease consisting of a history of PCI, CABG, MI or known coronary stenosis $>50 \%$; CABG: Coronary artery bypass grafting surgery; MI: Myocardial infarction; PAD: Peripheral artery disease; PCI: percutaneous coronary intervention; PPI: Protonpump inhibitor; TIA: Transient ischemic attack.
Supplementary Figure S5. Subgroup analyses for the combined primary bleeding outcome

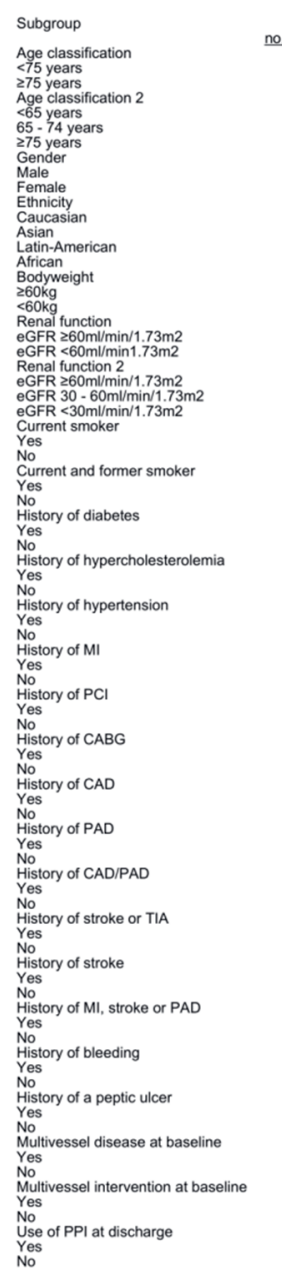

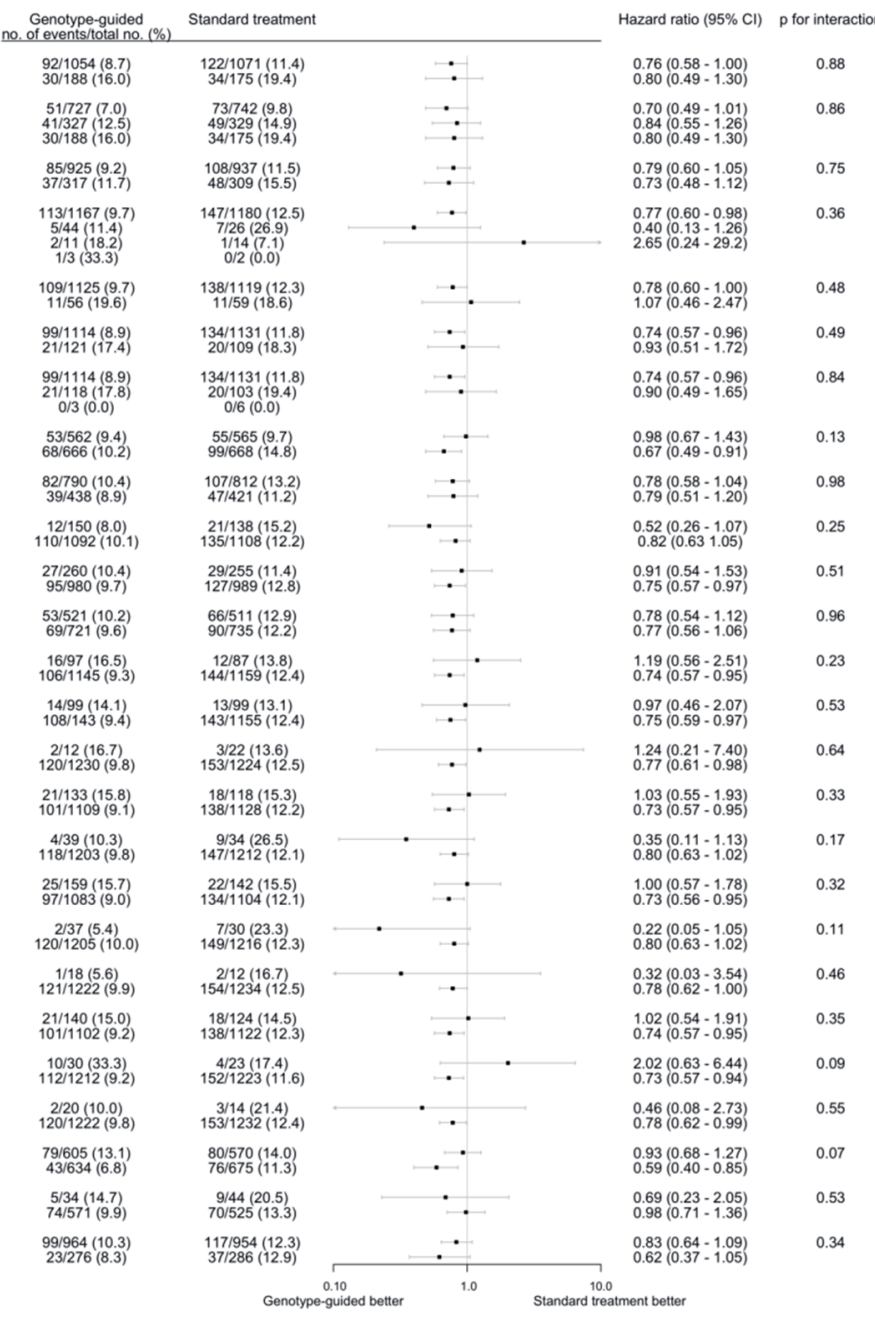

Analyses for 26 pre-specified subgroups for the primary bleeding outcome. eGFR: estimated glomerular filtration rate in $\mathrm{ml} / \mathrm{min} / 1.73 \mathrm{~m} 2$ calculated using the $\mathrm{CKD}$ EPI formula; CAD: coronary artery disease consisting of a history of PCI, CABG, MI or known coronary stenosis $>50 \%$; CABG: Coronary artery bypass grafting surgery; MI: Myocardial infarction; PAD: Peripheral artery disease; PCI: percutaneous coronary intervention; PPI: Proton-pump inhibitor; TIA: Transient ischemic attack. 


\section{Supplementary Table S1. Exclusion criteria}

- Unable to provide informed consent or have a life expectancy of less than one year

- Active malignancy causing an increased bleeding risk, at the investigator's opinion

- Women who are known to be pregnant or who have given birth within the past 90 days or who are breastfeeding

- Having received thrombolytic therapy within the previous 24 hours or oral anticoagulants during the previous 7 days

- Severe renal function impairment needing dialysis

- Confirmed or persistent severe hypertension (Systolic Blood Pressure $(\mathrm{SBP})>180 \mathrm{mmHg}$ and/or Diastolic Blood Pressure $>110 \mathrm{mmHg}$ ) at randomization

- Contraindication to anticoagulation or an increased bleeding risk, at the investigator's opinion

- $\quad$ Cardiogenic shock (SBP $\leq 80 \mathrm{mmHg}$ for $>30$ mins) or needing Intra-Aortic Balloon Pump

- History of major surgery, severe trauma, fracture or organ biopsy within 90 days prior to randomization

- Clinically significant out of range values for platelet count or haemoglobin at screening, at the investigator's opinion

\section{Supplementary Table S2. Secondary thrombotic outcomes}

- Composite end points of :

Vascular death, recurrent MI [2], definite stent thrombosis (ST) [3], stroke

- Vascular death, readmission for acute coronary syndrome (ACS), definite ST, stroke, urgent target vessel revascularization (uTVR)

- Individual efficacy outcome events:

○ All deaths (and the following sub-categories separately)

- Vascular death

- Readmission ACS (and the following sub-categories separately)

- Recurrent MI

Stroke (and the following sub-categories separately)

- Ischemic stroke

- Hemorrhagic stroke

- Definite ST (and the following sub-categories separately)

- Any ST (definite/probable/possible)

uTVR by either percutaneous coronary intervention or coronary artery bypass surgery 


\section{Supplementary Table S3. Secondary bleeding outcomes}

Platelet Inhibition and Patient Outcome (PLATO) bleeding [4]

- PLATO major bleeding

PLATO life threatening or fatal bleeding (and the following subcategories separately)

- Fatal bleeding

- Intracranial bleeding

- Fatal intracranial bleeding

- Nonfatal intracranial bleeding

- Non-CABG related PLATO major bleeding

Non-CABG related PLATO major + minor bleeding

- PLATO minor bleeding

- Bleeding Academic Research Consortium (BARC) bleeding [4]

BARC $3 / 4 / 5$

- BARC 3/5

- BARC $2 / 3 / 4 / 5$

BARC $2 / 3 / 5$

- BARC 2

- Thrombolysis in Myocardial Infarction (TIMI) bleeding [4]

- TIMI major bleeding

- TIMI major + minor bleeding

Non-CABG related TIMI major bleeding

- Non-CABG related TIMI major + minor bleeding

- TIMI minor bleeding

- Bleeding requiring Red Blood Cell transfusion

BARC: Bleeding academic research consortium; $\mathrm{CABG}$ : Coronary artery bypass grafting surgery; PLATO: Platelet Inhibition and Patient Outcomes; TIMI: Thrombolysis in Myocardial Infarction.

\section{Supplementary Table S4. Screenings log St. Antonius Hospital}

2508 patients screened in St. Antonius Hospital

1257 randomized

1251 excluded

311 - did not meet inclusion criteria

122 Symptoms $>12 \mathrm{~h}$

149 No stent

59 No ST-segment elevation myocardial infarction

940 - met an exclusion criterion

269 Not possible to obtain informed consent within $48 \mathrm{~h}$

91 Oral anticoagulation

129 Resuscitated

64 Cardiogenic shock or severe hypertension

37 Increased bleeding risk

30 Language barrier

84 Contraindication for aspirin or a $\mathrm{P} 2 \mathrm{Y}_{12}$ inhibitor

8 Life expectancy of $<1$ year

5 Recent major surgery

114 Declined to participate

62 Participated in competing trial

6 Other/unknown reason

15 Stroke\&

$\S$ Stroke was an exclusion criterion before May 2012. After the major protocol amendment, this was no longer an exclusion criterion. 

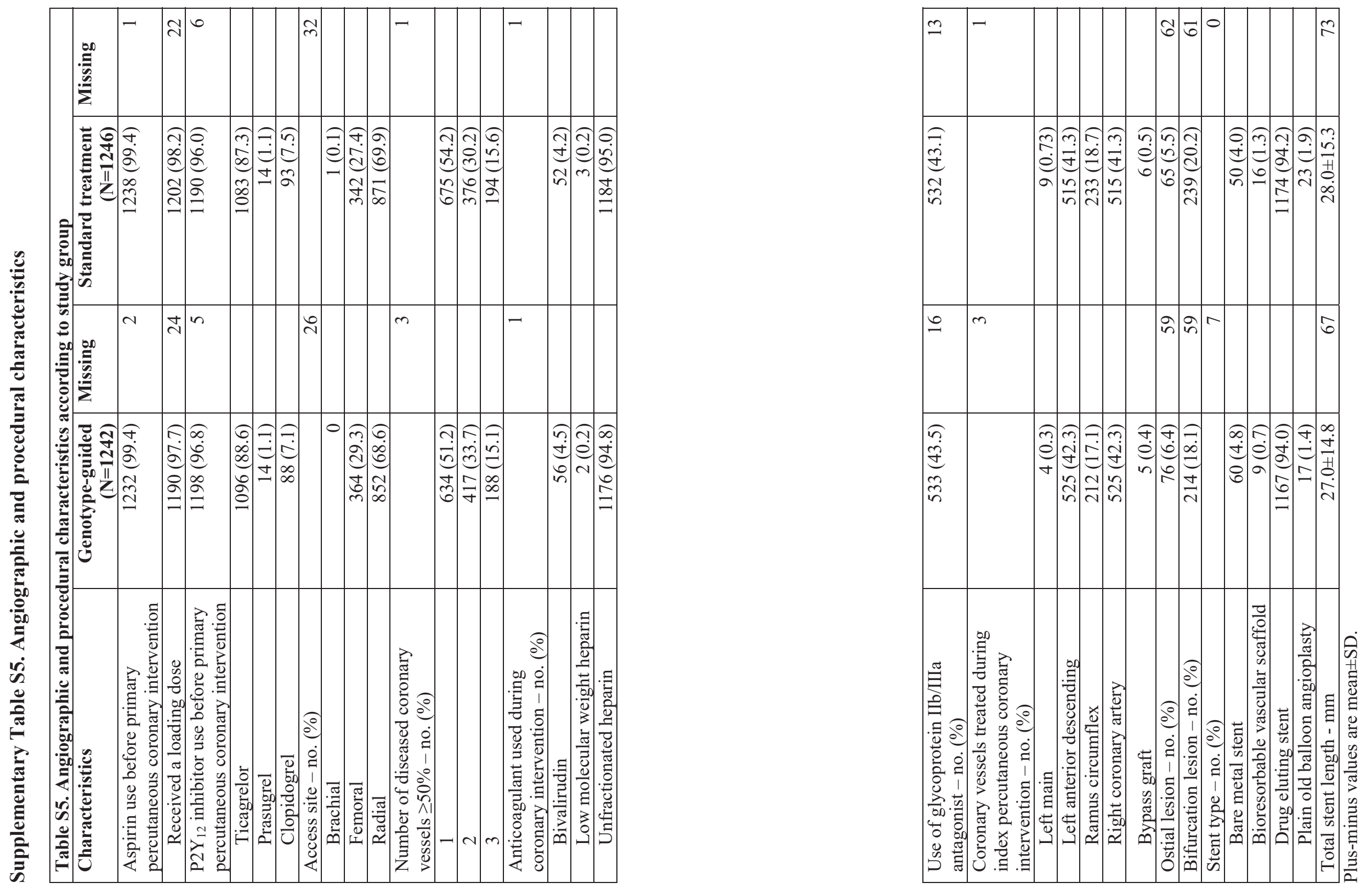
Supplementary Table S6. Characteristics at discharge

\begin{tabular}{|c|c|c|c|c|}
\hline Characteristics & $\begin{array}{r}\text { Genotype } \\
\text {-guided } \\
(\mathrm{N}=1242)\end{array}$ & Missing & $\begin{array}{c}\text { Standard } \\
\text { treatment } \\
(\mathrm{N}=1246)\end{array}$ & Missing \\
\hline $\begin{array}{l}\text { Left ventricular } \\
\text { ejection fraction - } \\
\text { no. }(\%)\end{array}$ & & 231 & & 211 \\
\hline Normal $(\geq 50 \%)$ & $578(57.2)$ & & $601(58.1)$ & \\
\hline $\begin{array}{l}\text { Moderately } \\
\text { reduced ( } \geq 30 \% \text { - } \\
<50 \%)\end{array}$ & $407(40.3)$ & & $408(39.4)$ & \\
\hline $\begin{array}{l}\text { Severely reduced } \\
(<30 \%)\end{array}$ & $26(2.6)$ & & $26(2.5)$ & \\
\hline \multicolumn{5}{|l|}{$\begin{array}{l}\text { Other Medication at } \\
\text { discharge - no. }(\%)\end{array}$} \\
\hline Beta blocker & $\begin{array}{r}1110 \\
(89.5) \\
\end{array}$ & 0 & 1099 (88.6) & 0 \\
\hline ACE inhibitor & $942(76.0)$ & 0 & $966(77.9)$ & 0 \\
\hline $\begin{array}{l}\text { Angiotensin-II- } \\
\text { receptor blocker }\end{array}$ & $119(9.6)$ & 0 & $109(8.8)$ & 0 \\
\hline $\begin{array}{l}\text { Calcium-channel } \\
\text { inhibitor }\end{array}$ & $129(10.4)$ & 0 & $121(9.8)$ & 0 \\
\hline $\begin{array}{l}\text { Proton-pump } \\
\text { inhibitor }\end{array}$ & $964(77.7)$ & 0 & $954(76.9)$ & 0 \\
\hline Statin treatment & $\begin{array}{r}1207 \\
(97.3)\end{array}$ & 0 & $1200(96.8)$ & 0 \\
\hline
\end{tabular}

Supplementary Table S7. Prevalence of genetic variants in the genotype-guided group

\begin{tabular}{|l|r|}
\hline Genotype & $\begin{array}{l}\text { Number of } \\
\text { patients (\%) }\end{array}$ \\
\hline$* 1 / * 1$ & $835(67.2)$ \\
\hline$* 1 / * 2$ & $353(28.4)$ \\
\hline$* 1 / * 3$ & $4(0.3)$ \\
\hline$* 2 / * 2$ & $30(2.4)$ \\
\hline$* 2 / * 3$ & $2(0.2)$ \\
\hline$* 3 / * 3$ & 0 \\
\hline Not available & $18(1.4)$ \\
\hline
\end{tabular}

*1/*1: Extensive metabolizer;

$* 1 / * 2$ and $* 1 / * 3$ : Intermediate metabolizer;

$* 2 / * 2 . * 2 / * 3$ and $* 3 / * 3$ : Poor metabolizer. 
Supplementary Tables S9A, S9B \& S9C. Discontinuing and switching $\mathrm{P} \mathrm{Y}_{12}$ inhibitor therapy

Table 9A. Reasons for discontinuing or switching $\mathrm{P}_{2} \mathrm{Y}_{12}$ inhibitors

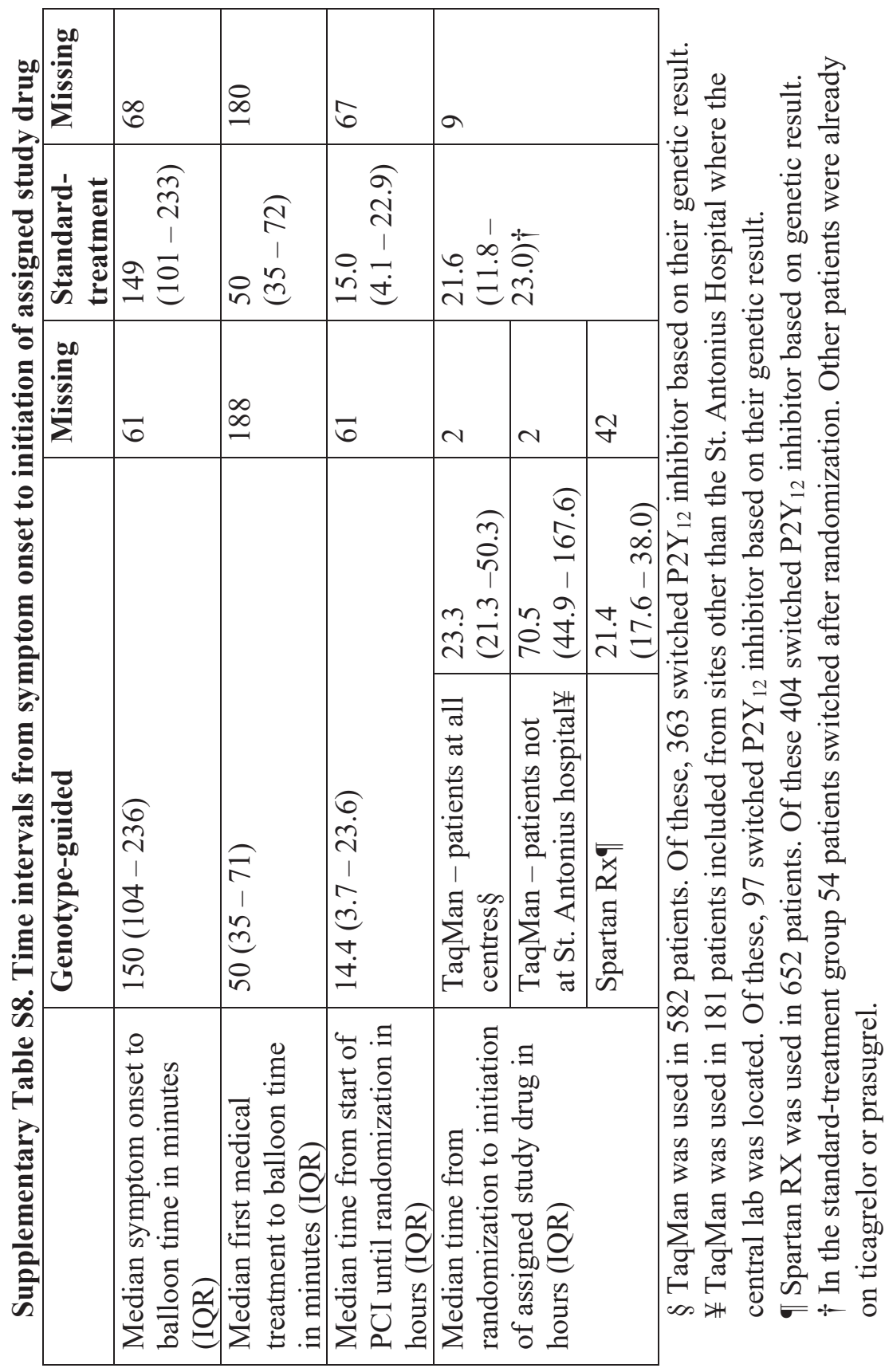

\begin{tabular}{|c|r|r|}
\hline & Genotype-guided (N) & Standard treatment (N) \\
\hline Total & 126 & 256 \\
\hline Side effects & 60 & 123 \\
\hline CABG & 13 & 27 \\
\hline OAC & 19 & 54 \\
\hline Other & 24 & 30 \\
\hline Unknown & 10 & 22 \\
\hline
\end{tabular}

One patient can have switched multiple times. CABG: Coronary Artery Bypass Grafting surgery; OAC: Oral Anticoagulants.

Table 9B. Overview of switches

\begin{tabular}{|l|r|r|}
\hline Switches & $\begin{array}{l}\text { Genotype- } \\
\text { guided (N) }\end{array}$ & $\begin{array}{l}\text { Standard } \\
\text { treatment (N) }\end{array}$ \\
\hline Ticagrelor to clopidogrel & 65 & 199 \\
\hline Ticagrelor to prasugrel & 13 & 22 \\
\hline Ticagrelor to no P2Y 12 inhibitor & 15 & 20 \\
\hline Clopidogrel to ticagrelor & 16 & 4 \\
\hline Clopidogrel to prasugrel & 2 & 0 \\
\hline $\begin{array}{l}\text { Clopidogrel to no P2Y } \\
\text { inhibitor }\end{array}$ & 10 & 5 \\
\hline Prasugrel to ticagrelor & & 1 \\
\hline Prasugrel to clopidogrel & 0 & 3 \\
\hline Prasugrel to no P2Y 12 inhibitor & 0 & 0 \\
\hline Other & 1 & 2 \\
\hline
\end{tabular}

Table 9C. Side Effects Causing Patients to Switch

\begin{tabular}{|l|r|r|r|}
\hline & \multicolumn{1}{|l|}{ Ticagrelor } & \multicolumn{1}{|c|}{ Prasugrel } & Clopidogrel \\
\hline Dyspnea & 106 & 0 & 1 \\
\hline Bleeding & 28 & 1 & 4 \\
\hline $\begin{array}{l}\text { Gastro-intestinal } \\
\text { bleeding }\end{array}$ & 9 & 0 & 2 \\
\hline Itching or rash & 10 & 2 & 3 \\
\hline Fatigue & 13 & 0 & 1 \\
\hline Other/unknown & 11 & 1 & 1 \\
\hline
\end{tabular}

Some switches had more than one cause. 

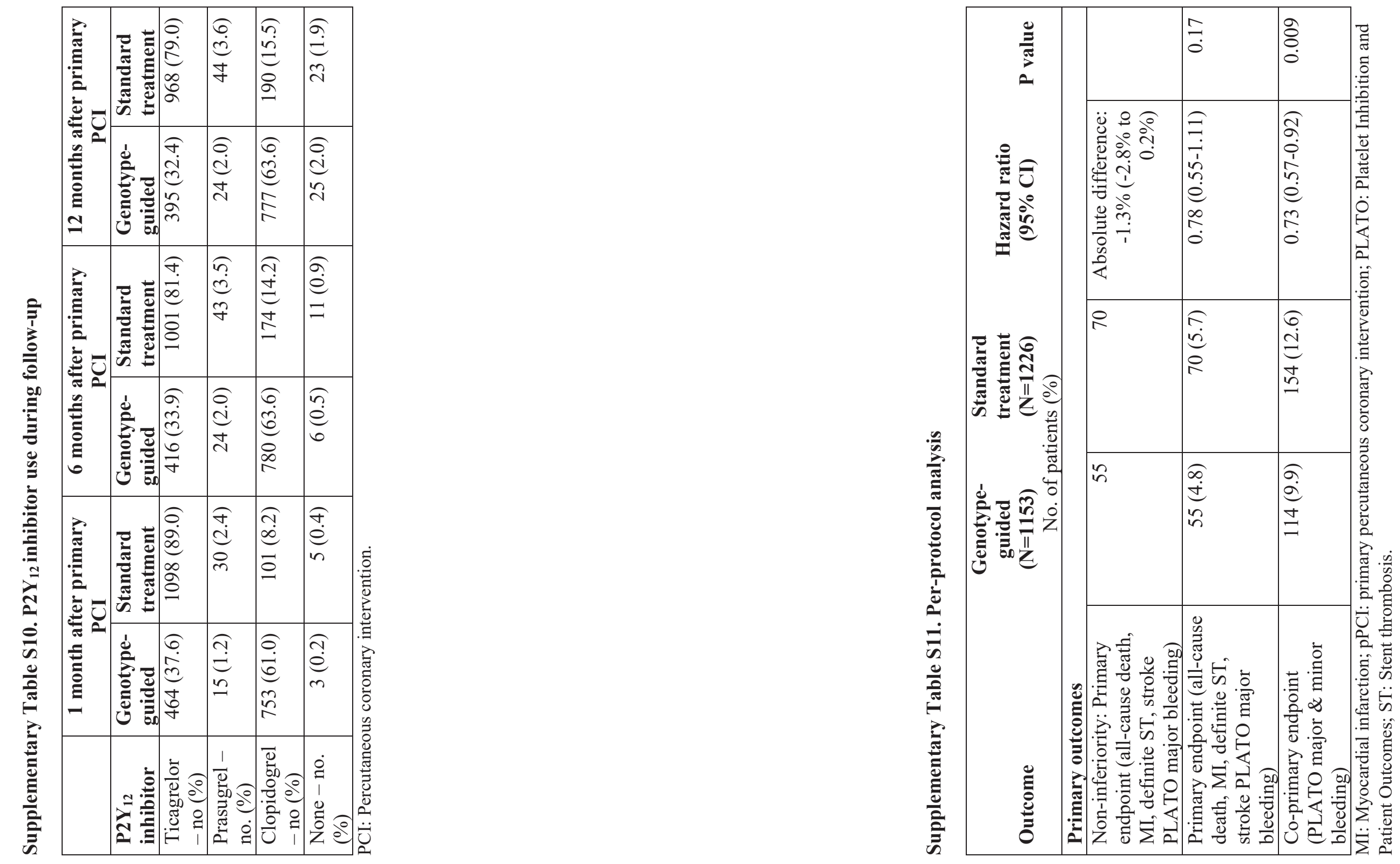
Supplementary Table S12. Sensitivity analyses

\begin{tabular}{|c|c|c|c|c|}
\hline Outcome & \begin{tabular}{l} 
Genotype- \\
guided \\
$(\mathrm{N}=1242)$ \\
\multicolumn{1}{c}{ No. of }
\end{tabular} & $\begin{array}{l}\text { Standard } \\
\text { treatment } \\
(\mathbf{N}=1246) \\
\text { patients }(\%)\end{array}$ & $\begin{array}{l}\text { Hazard ratio } \\
(95 \% \text { CI) }\end{array}$ & $\begin{array}{l}P \\
\text { value }\end{array}$ \\
\hline Including events & in the 24 hour & after pPCI & & \\
\hline $\begin{array}{l}\text { Non-inferiority: } \\
\text { Primary } \\
\text { endpoint (all- } \\
\text { cause death, } \\
\text { MI, definite ST, } \\
\text { stroke PLATO } \\
\text { major bleeding) }\end{array}$ & 70 & 83 & $\begin{array}{r}\text { Absolute } \\
\text { difference: } \\
-0.9 \% \\
(-2.5 \% \text { to } \\
0.6 \%)\end{array}$ & \\
\hline $\begin{array}{l}\text { Primary } \\
\text { endpoint (all- } \\
\text { cause death, } \\
\text { MI, definite ST, } \\
\text { stroke PLATO } \\
\text { major bleeding) }\end{array}$ & $70(5.6)$ & $83(6.7)$ & $\begin{array}{r}0.84 \\
(0.61-1.16)\end{array}$ & 0.29 \\
\hline $\begin{array}{l}\text { Co-primary } \\
\text { endpoint } \\
\text { (PLATO major } \\
\& \text { minor } \\
\text { bleeding) } \\
\end{array}$ & $151(12.2)$ & $190(15.2)$ & $\begin{array}{r}0.78 \\
(0.63-0.96)\end{array}$ & 0.02 \\
\hline \multicolumn{5}{|c|}{ Using stratification per center } \\
\hline $\begin{array}{l}\text { Primary } \\
\text { endpoint (all- } \\
\text { cause death, } \\
\text { MI, definite ST, } \\
\text { stroke PLATO } \\
\text { major bleeding) }\end{array}$ & $63(5.1)$ & $73(5.9)$ & $\begin{array}{r}0.86 \\
(0.62-1.21)\end{array}$ & 0.40 \\
\hline $\begin{array}{l}\text { Co-primary } \\
\text { endpoint } \\
\text { (PLATO major } \\
\& \text { minor } \\
\text { bleeding) }\end{array}$ & $122(9.8)$ & $156(12.5)$ & $\begin{array}{r}0.76 \\
(0.60-0.97)\end{array}$ & 0.03 \\
\hline
\end{tabular}

MI: Myocardial infarction; pPCI: primary percutaneous coronary intervention;

PLATO: Platelet Inhibition and Patient Outcomes; ST: Stent thrombosis.

\section{Supplementary appendix references}

1. Hamm CW, Bassand JP, Agewall S, et al. ESC guideline for the management of acute coronary syndromes in patients presenting without persistent ST-segment elevation. Eur Heart J 2011; 32:2999-3054.

2. Thygesen K, Alpert JS, Jaffe AS, et al. Third universal definition of myocardial infarction. J Am Coll Cardiol 2012; 60:1581-98.

3. Cutlip DE, Windecker S, Mehran R, et al. Clinical end points in coronary stent trials: a case for standardized definitions. Circulation 2007; 115:2344-51.

4. Mehran R, Rao SV, Bhatt DL, et al. Standardized bleeding definitions for cardiovascular clinical trials: a consensus report from the bleeding academic research consortium. Circulation 2011; 123:2736-47. 
Clopidogrel versus ticagrelor or prasugrel after primary percutaneous coronary intervention according to CYP2C19 genotype. A POPular genetics subanalysis

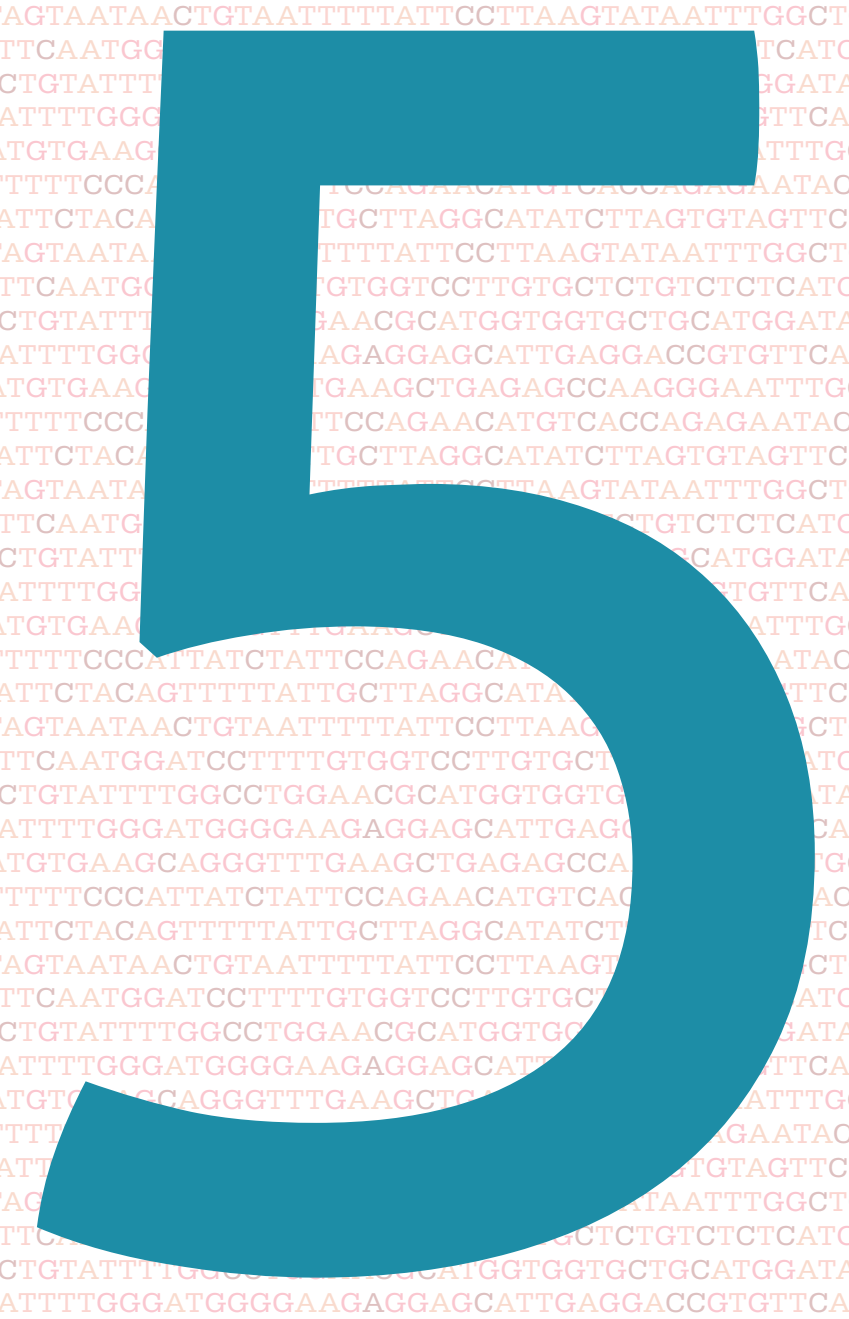

Daniel M.F. Claassens, Thomas 0. Bergmeijer, Gerrit J.A. Vos, , Renicus S. Hermanides, Arnoud W.J. van't Hof, Pim van der Harst, Emanuele Barbato, Carmine Morisco, Richard M. Tjon Joe Gin, Folkert W. Asselbergs, Arend Mosterd, Jean-Paul R. Herrman Willem J.M. Dewilde, Paul W.A. Janssen, Johannes C. Kelder, Bakhtawar K. Mahmoodi, Vera H.M.Deneer, Jurriën M. ten Berg

Circulation: Cardiovascular Interventions 2021; $14: \mathrm{e} 009434$

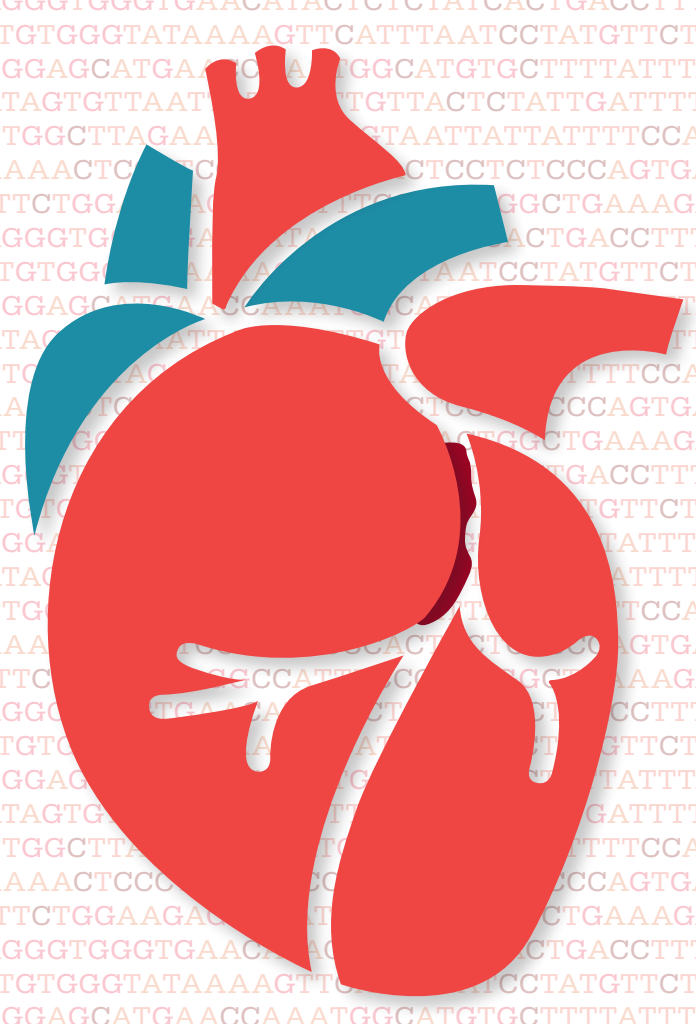




\section{Abstract}

\section{Background}

Guidelines favour ticagrelor or prasugrel over clopidogrel in patients with myocardial infarction. However, the POPular Genetics trial (Patient Outcome After Primary Percutaneous Coronary Intervention [PCI]) showed that in patients with primary PCI, a CYP2C19 genotype-guided strategy was associated with a lower bleeding risk without increasing thrombotic risk, compared with routine ticagrelor/prasugrel treatment. Nevertheless, optimal $\mathrm{P}_{2} \mathrm{Y}_{12}$ inhibitor treatment in specific CYP2C19 genetic subgroups is still a subject of debate.

\section{Methods}

A prespecified subanalysis of the POPular Genetics trial was performed, using patients in whom $C Y P 2 C 19 * 2, * 3$, and $* 17$ genotypes was determined. Two different analyses were planned. The first assessed the effect of the CYP $2 C 19 * 17$ allele in clopidogrel-treated patients. The second compared the effect of clopidogrel in noncarriers of a loss-offunction allele with ticagrelor/prasugrel-treated patients, irrespective of CYP2C19 genotype. Main outcomes were a thrombotic outcome (cardiovascular death, myocardial infarction, stent thrombosis, and stroke) and a bleeding outcome (PLATO [Platelet Inhibition and Patient Outcomes] major and minor bleeding) after 12 months.

\section{Results}

A total of 2429 patients were used for analyses. In the first analysis, the CYP2C19*17 polymorphism was not found to have a significant influence on thrombotic (adjusted hazard ratio, 0.95 [95\% CI, 0.45-2.02]) or bleeding outcomes (adjusted hazard ratio, 0.74 [95\% CI, 0.48-1.18]). In the second analysis, clopidogrel was associated with a lower number of bleeding events compared with ticagrelor/prasugrel (9.9\% versus $11.7 \%$, adjusted hazard ratio, 0.74 [95\% CI, 0.56-0.96]), without a significant increase in thrombotic events $(3.4 \%$ versus $2.5 \%$, adjusted hazard ratio, 1.14 [95\% CI, 0.68-1.90]).

\section{Conclusions}

In patients with primary PCI not carrying a CYP2C19 loss-of-function allele, the use of clopidogrel compared with ticagrelor or prasugrel was associated with lower bleeding rates, without an increase in thrombotic events. No effect on clinical outcomes was found for the CYP2C19*17 polymorphism.

\section{Introduction}

Dual antiplatelet therapy, consisting of aspirin and a $\mathrm{P} 2 \mathrm{Y}_{12}$ inhibitor remains the cornerstone of antithrombotic treatment in patients with myocardial infarction. Guidelines favour ticagrelor and prasugrel because these showed a reduction in thrombotic events as compared with clopidogrel [1,2]. This higher efficacy, however, is hampered by a higher bleeding risk $[3,4]$

Clopidogrel is a prodrug, transformed into its active metabolite by hepatic cytochrome P450 enzymes in the liver. There is a large variability in antiplatelet effect in patients treated with clopidogrel, which in part can be explained by genetic variations in the CYP2C19 gene [5]. Patients carrying a $C Y P 2 C 19 * 2$ or $* 3$ loss-of-function (LoF) allele are at higher risk for having high on-treatment platelet reactivity when treated with clopidogrel, which is associated with a higher risk of stent thrombosis and recurren atherothrombotic events [6]. In patients carrying a CYP2C19*17 gain-offunction allele, clopidogrel efficacy might be better, with possibly higher bleeding rates $[7,8]$. However, data on the influence of CYP2C19*17 on clinical outcome are conflicting and mostly derived from observational studies [9]. Its clinical relevance is, therefore, not clear.

In the recently published POPular Genetics trial (Patient Outcome After Primary Percutaneous Coronary Intervention [PCI]), a CYP2C19 genotype-guided antiplatelet strategy, using clopidogrel in patients without LoF allele and ticagrelor or prasugrel in patients carrying $\geq 1 \mathrm{LoF}$ alleles, showed a reduction in bleeding risk (defined as PLATO [Platelet Inhibition and Patient Outcomes] major or minor bleeding) compared with standard treatment with ticagrelor or prasugrel, without an increase in thrombotic risk [10]. Moreover, in a subanalysis of the PLATO trial, ticagrelor was not superior to clopidogrel regarding the thrombotic outcome [11]. Since then, thrombotic event rates have declined considerably [12]. At the same time, the incidence of bleeding events, which are associated with a substantial morbidity and mortality risk, remained high [13]. Therefore, the question of which antiplatelet strategy is best to balance thrombotic and bleeding risk in patients with STsegment-elevation myocardial infarction after primary PCI is still open, in particular for different subgroups of noncarriers of CYP2C19 LoF alleles.

In this prespecified subanalysis of the POPular Genetics trial, we study the effect of the CYP2C19*17 allele in clopidogrel-treated patients and the safety and efficacy of clopidogrel in patients without an LoF allele, compared with ticagrelor and prasugrel. 
Figure 1. Flowchart of included patients

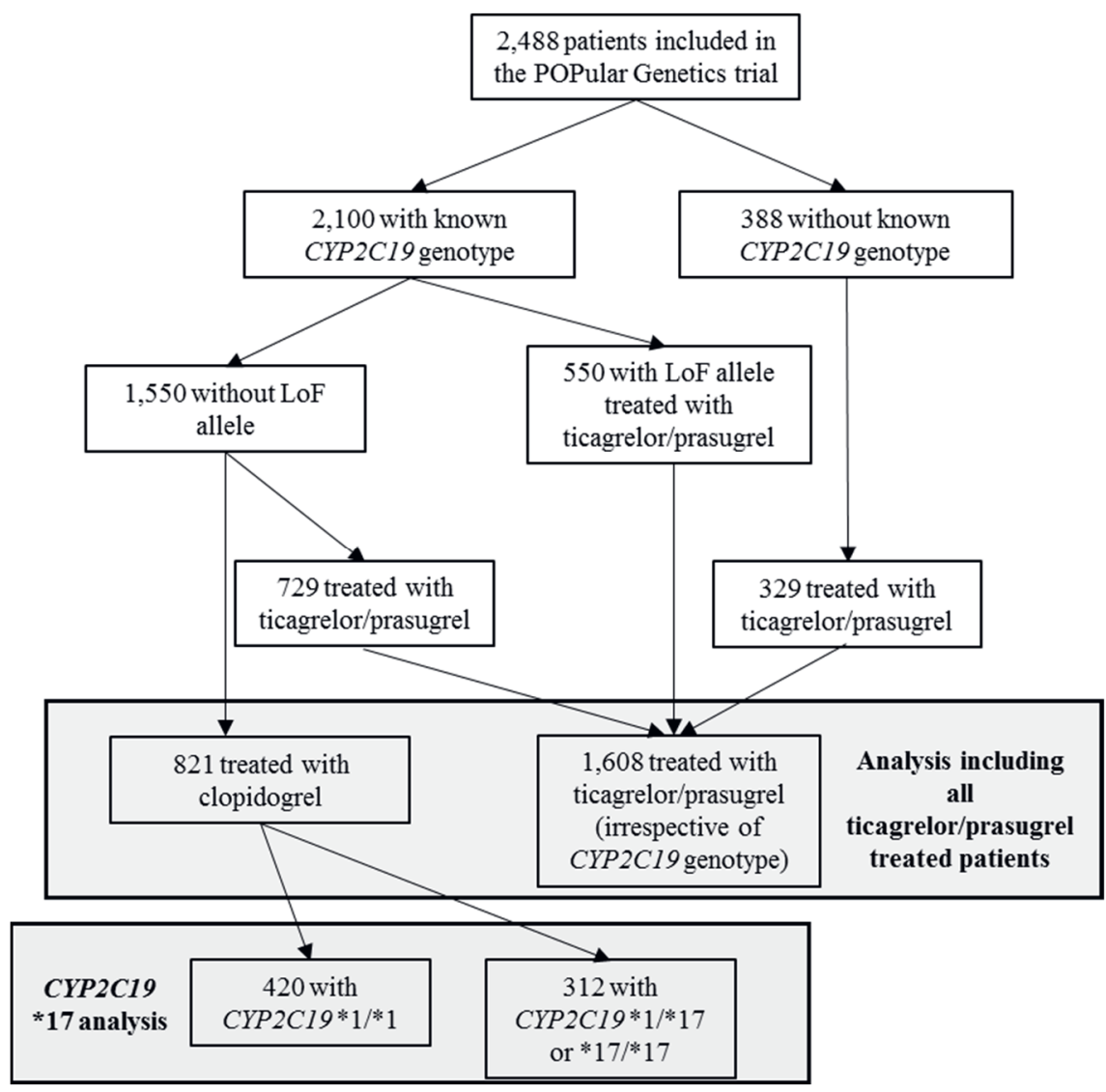

Figure 1. Flowchart of patients included in the different subgroup analyses. LoF indicates loss-of-function

\section{Methods}

Study design

The detailed design and results of the POPular Genetics trial have been reported previously $[10,14]$. In brief, POPular Genetics was an open-label, randomized, multicentre trial with 2488 patients with ST-segmentelevation myocardial infarction undergoing primary PCI. It compared a standard treatment with ticagrelor or prasugrel with a CYP2C19 genotypeguided strategy, where patients received clopidogrel if they did not carry a CYP2C19*2 or *3 LoF allele and ticagrelor or prasugrel if they were carrier of such a LoF allele. Treatment and follow-up duration were 12 months. An institutional review board approved the trial, and all participants provided informed consent.

Blood samples were collected from patients in both treatment groups. During the trial, CYP2C19 genotyping was performed in the genotypeguided group, where the presence of the CYP2C19*2 and *3 polymorphism was determined using the Spartan RX point-of-care system or the TaqMan StepOnePlus assay. After the trial was completed, genotyping was performed in all patients in the standard treatment arm, and both groups were tested for CYP2C19*17. This was done by LGC Biosearch Technologies (Hoddesdon, United Kingdom) using a competitive allele-specific genotyping assay. For the analyses, an intention to treat analysis was performed in which patients were divided into a clopidogrel-treated or ticagrelor/prasugrel-treated group, based on the drug prescribed at discharge. Two different analyses were performed. The first analysis compared clopidogrel in CYP2C19*1/*1 patients with clopidogrel in patients with a $* 1 / * 17$ or $* 17 / * 17$ genotype. No separate analysis for homozygous CYP2C19*17 carriers was performed because of the low number of patients in this group $(n=35)$. The second analysis compared clopidogrel-treated patients not carrying a CYP $2 C 19 * 2$ or $* 3$ LoF allele with ticagrelor- or prasugrel-treated patients irrespective of their CYP2C19 metabolizer status. In addition, we performed 2 sensitivity analyses, one including only patients without LoF alleles in both the clopidogrel and ticagrelor- or prasugrel-treated group and one on-treatment analysis.

Table 1. (see next page) ACE: angiotensin-converting enzyme; ATII, angiotensin II; BMI, body mass index; *Creatinine clearance was calculated using the CKDEPI formula 
Table 1. Baseline characteristics of clopidogrel-treated patients according to $C Y P 2 C 19 * 17$ carrier status

\begin{tabular}{|c|c|c|c|}
\hline & $\begin{array}{l}\text { CYP2C19*1/*1; } \\
\mathrm{N}=420\end{array}$ & $\begin{array}{l}\text { CYP2C19*17 } \\
\text { carriers; } \mathrm{N}=312\end{array}$ & $P$ value \\
\hline Age, $y$, mean \pm SD & $61.2 \pm 10.8$ & $62.8 \pm 11.2$ & 0.06 \\
\hline Female sex, n (\%) & $105(25.0)$ & $89(28.5)$ & 0.33 \\
\hline $\mathrm{BMI}, \mathrm{kg} / \mathrm{m} 2$, mean $\pm \mathrm{SD}$ & $27.7 \pm 9.85$ & $27.1 \pm 4.14$ & 0.28 \\
\hline $\begin{array}{l}\text { Creatinine clearance }<60 \\
\mathrm{~mL} / \mathrm{min} / 1.73 \mathrm{~m} 2 \text { at } \\
\text { baseline, }{ }^{*} \mathrm{n}(\%)\end{array}$ & $39(9.4)$ & $29(9.4)$ & 1.0 \\
\hline \multicolumn{4}{|l|}{ History, $\mathrm{n}(\%)$} \\
\hline Current smoker & $199(48.2$ & $126(40.6)$ & 0.05 \\
\hline Hypertension & $174(41.4)$ & $127(40.7)$ & 0.90 \\
\hline Dyslipidaemia & $83(19.8)$ & $63(20.2)$ & 0.96 \\
\hline Diabetes & $43(10.2)$ & $32(10.3)$ & 1.0 \\
\hline Coronary artery disease & $47(11.2)$ & $32(10.3)$ & 0.78 \\
\hline Peripheral arterial disease & $17(4.1)$ & $11(3.5)$ & 0.87 \\
\hline Stroke & $7(1.7)$ & $3(1.0)$ & 0.53 \\
\hline Bleeding & $9(2.1)$ & $8(2.6)$ & 0.90 \\
\hline \multicolumn{4}{|c|}{ Discharge medication, $\mathrm{n}(\%)$} \\
\hline Aspirin & $404(96.4)$ & $302(96.8)$ & 0.94 \\
\hline \multicolumn{4}{|c|}{$\mathrm{P}^{2} \mathrm{Y}_{12}$ inhibitor after discharge } \\
\hline Clopidogrel & $420(100)$ & $312(100)$ & \\
\hline Vitamin $\mathrm{K}$ antagonist & $26(6.2)$ & $13(4.2)$ & 0.30 \\
\hline Novel anticoagulant & $6(1.4)$ & $8(2.6)$ & 0.41 \\
\hline ACE inhibitor & $320(76.4)$ & $227(72.8)$ & 0.30 \\
\hline ATII antagonist & $42(10.0)$ & $33(10.6)$ & 0.90 \\
\hline Beta blocker & $370(88.3)$ & $276(88.5)$ & 1.0 \\
\hline Statin & $408(97.4)$ & $300(96.2)$ & 0.47 \\
\hline Proton pump inhibitor & $332(79.2)$ & $247(79.2)$ & 1.0 \\
\hline \multicolumn{4}{|l|}{ Procedural characteristics } \\
\hline Access site & & & 0.54 \\
\hline Femoral & $133(31.7)$ & $106(34.2)$ & \\
\hline Radial & $286(68.3)$ & $204(65.8)$ & \\
\hline Multivessel disease & $205(48.8)$ & $168(53.8)$ & 0.20 \\
\hline \multicolumn{4}{|l|}{ Stent } \\
\hline None & $10(2.4)$ & $4(1.3)$ & 0.42 \\
\hline Bare metal stent & $21(5.0)$ & $20(6.4)$ & 0.51 \\
\hline Biovascular scaffold & $4(0.1)$ & $3(1.0)$ & 1.0 \\
\hline Drug eluting stent & $393(93.6)$ & $292(93.6)$ & 1.0 \\
\hline Ostial lesion & $28(6.7)$ & $20(6.5)$ & 1.0 \\
\hline Bifurcation lesion & $82(19.6)$ & $64(20.6)$ & 0.79 \\
\hline $\begin{array}{l}\text { Total stent length, mm, } \\
\text { mean } \pm \text { SD }\end{array}$ & $27.6 \pm 14.0$ & $26.6 \pm 13.3$ & 0.33 \\
\hline
\end{tabular}

Table 2. Baseline characteristics of clopidogrel in patients without CYP2C19*2 or *3 LoF allele versus ticagrelor or prasugrel in all patients (irrespective of CYP2C19 genotype).

\begin{tabular}{|c|c|c|c|}
\hline & $\begin{array}{l}\text { Clopidogrel } \\
\text { (no LoF } \\
\text { allele); } \mathbf{N}=821\end{array}$ & $\begin{array}{l}\text { Ticagrelor } \\
\text { and } \\
\text { prasugrel; } \\
\mathrm{N}=1608\end{array}$ & $P$ value \\
\hline Age, y, mean \pm SD & $62.0 \pm 10.9$ & $61.3 \pm 11.3$ & 0.14 \\
\hline Female sex, n (\%) & $224(27.3)$ & $388(24.1)$ & 0.10 \\
\hline BMI, kg/m2, mean \pm SD & $27.5 \pm 7.66$ & $27.2 \pm 4.22$ & 0.32 \\
\hline $\begin{array}{l}\text { Creatinine clearance }<60 \\
\mathrm{~mL} /(\mathrm{min} \cdot 1.73 \mathrm{~m} 2) \text { at } \\
\text { baseline, }{ }^{*} \mathrm{n}(\%)\end{array}$ & $75(9.2)$ & $148(9.2)$ & 1.0 \\
\hline \multicolumn{4}{|l|}{ History, n (\%) } \\
\hline Current smoker & $372(45.9)$ & $738(46.3)$ & 0.90 \\
\hline Hypertension & $348(42.4)$ & $655(40.7)$ & 0.46 \\
\hline Dyslipidaemia & $172(21.0)$ & $332(20.7)$ & 0.91 \\
\hline Diabetes & $89(10.8)$ & $186(11.6)$ & 0.64 \\
\hline Coronary artery disease & $82(10.0)$ & $157(9.8)$ & 0.92 \\
\hline $\begin{array}{l}\text { Peripheral arterial } \\
\text { disease }\end{array}$ & $31(3.8)$ & $41(2.6)$ & 0.12 \\
\hline Stroke & $10(1.2)$ & $20(1.2)$ & 1.0 \\
\hline Bleeding & $19(2.3)$ & $33(2.1)$ & 0.78 \\
\hline \multicolumn{4}{|c|}{ Discharge medication, $\mathrm{n}(\%)$} \\
\hline Aspirin & $794(96.8)$ & $1578(98.3)$ & 0.03 \\
\hline \multicolumn{4}{|c|}{$\mathrm{P}^{2} \mathrm{Y}_{12}$ inhibitor after discharge } \\
\hline Clopidogrel & $821(100)$ & $0(0.0)$ & \\
\hline Prasugrel 10mg & $0(0.0)$ & $38(2.4)$ & \\
\hline Prasugrel 5mg & $0(0.0)$ & $5(0.3)$ & \\
\hline Ticagrelor & $0(0.0)$ & $1565(97.3)$ & \\
\hline Vitamin $\mathrm{K}$ antagonist & $42(5.1)$ & $22(1.4)$ & $<0.001$ \\
\hline Novel anticoagulant & $14(1.7)$ & $3(0.2)$ & $<0.001$ \\
\hline ACE inhibitor & $618(75.4)$ & $1252(78.0)$ & 0.16 \\
\hline ATII antagonist & $77(9.4)$ & $147(9.2)$ & 0.91 \\
\hline Beta blocker & $726(88.5)$ & $1435(89.4)$ & 0.56 \\
\hline Statin & $795(97.0)$ & $1560(97.2)$ & 0.83 \\
\hline Proton pump inhibitor & $656(80.0)$ & $1217(75.8)$ & 0.02 \\
\hline \multicolumn{4}{|l|}{ Procedural characteristics } \\
\hline Access site & & & 0.23 \\
\hline Femoral & $249(31.1)$ & $444(28.2)$ & \\
\hline Radial & $552(68.9)$ & $1127(71.7)$ & \\
\hline Multivessel disease & $402(49.1)$ & $739(46.0)$ & 0.17 \\
\hline Stent & & & \\
\hline
\end{tabular}




\begin{tabular}{|l|l|l|l|}
\hline None & $14(1.7)$ & $25(1.6)$ & 0.91 \\
\hline Bare metal stent & $44(5.4)$ & $62(3.9)$ & 0.11 \\
\hline Biovascular scaffold & $8(1.0)$ & $17(1.1)$ & 1.0 \\
\hline Drug eluting stent & $766(93.3)$ & $1520(94.5)$ & 0.26 \\
\hline Ostial lesion & $53(6.8)$ & $85(5.6)$ & 0.27 \\
\hline Bifurcation lesion & $154(19.8)$ & $287(18.7)$ & 0.59 \\
\hline $\begin{array}{l}\text { Total stent length, mm, } \\
\text { mean } \pm \text { SD }\end{array}$ & $27.3 \pm 13.7$ & $28.8 \pm 17.8$ & 0.07 \\
\hline
\end{tabular}

Table 2. (see previous page) ACE: angiotensin-converting enzyme; ATII, angiotensin II; BMI, body mass index. *Creatinine clearance was calculated using the CKD-EPI formula.

\section{Statistical analysis}

This prespecified subanalysis was not prospectively powered and is based on the number of patients in the original trial of whom the CYP2C19 genetic profile was available. The outcomes were a thrombotic outcome consisting of cardiovascular death, myocardial infarction, stent thrombosis and stroke, a bleeding outcome consisting of PLATO major and minor bleeding, and the individual components of the thrombotic and bleeding outcome. The definitions were identical to those used in the POPular Genetics trial [10]. All outcomes were adjudicated by a blinded event committee.

Variables are presented as numbers (percentages) and mean \pm SD. Missing baseline variables were not imputed. $\mathrm{P}$ values were calculated using Student $t$ test for continuous variables, $\chi 2$ tests for categorical variables, and 1-way ANOVAs for variables with multiple categories. A P value below 0.05 was considered statistically significant. Kaplan-Meier curves were estimated and the log-rank test was used to calculate $\mathrm{P}$ values. If a patient was lost to follow-up, it was censored after the last known contact. Cox proportional hazard models were used to calculate hazard ratios (HR) and the $95 \%$ CI. The Efron approximation was used to handle ties. To adjust for possible confounders, all baseline characteristics with a $\mathrm{P}<0.10$ were selected for univariate regression analysis. If there was a significant interaction $(\mathrm{P}<0.05)$ in the univariate analysis, they were selected for multivariable regression analysis. The final model included only those characteristics with a significant interaction in the multivariable analysis. Main effects were included in the model. All analyses were performed using $\mathrm{R}$ version 3.6.0.

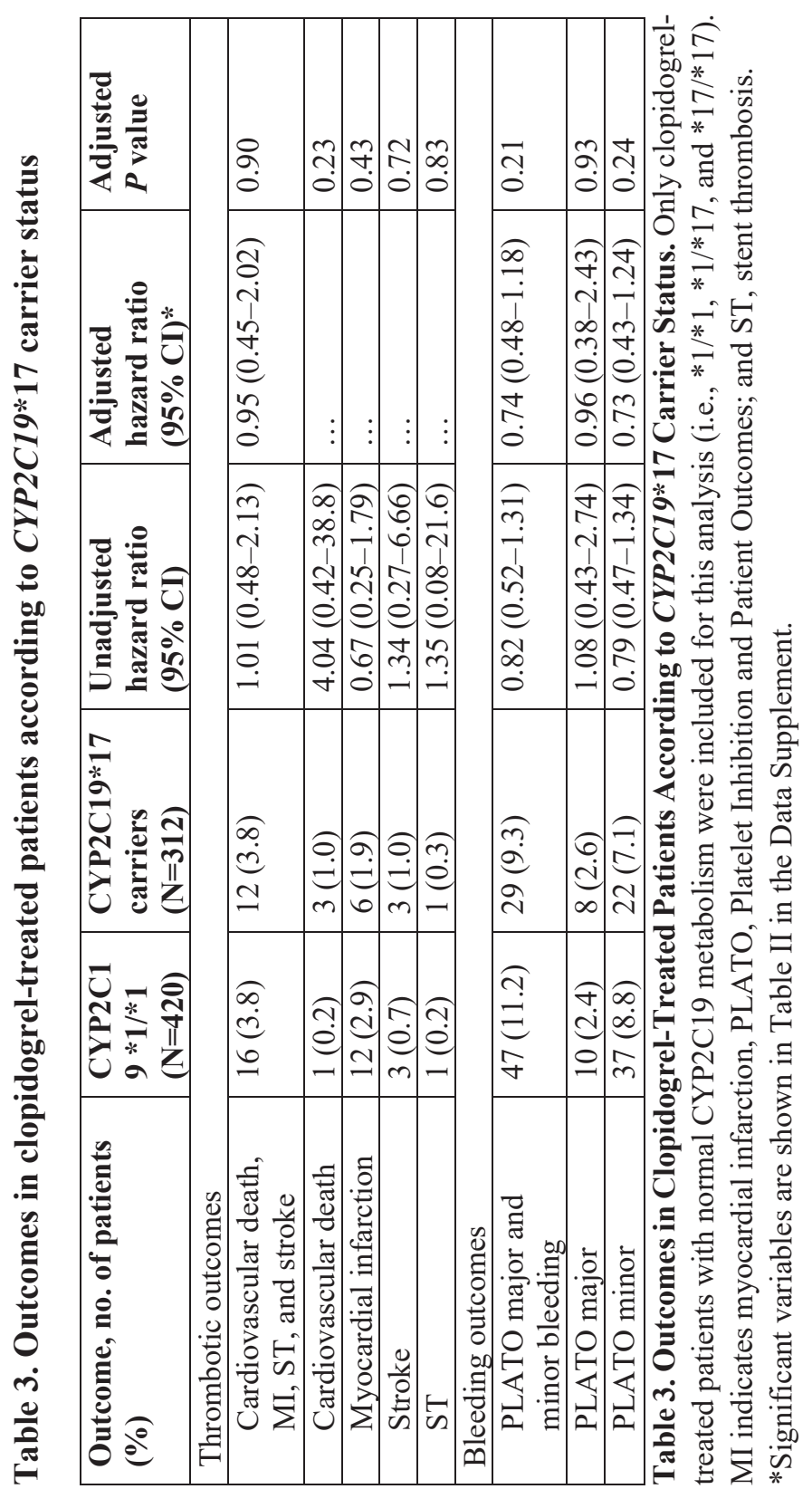




\section{Results}

Figure 1 shows a flow chart of the selection process of the subgroups. In the POPular Genetics cohort, CYP2C19*2,*3, and *17 carrier status was available in 2100 patients, of which 1550 did not carry a LoF allele. In the remaining 388 patients, CYP2C19 carrier status was unknown. Of these 388 patients, 329 were treated with ticagrelor or prasugrel and could therefore be included in the ticagrelor/prasugrel group of the second analysis. Of the 1550 patients not carrying a LoF allele, 821 were treated with clopidogrel and 729 with ticagrelor or prasugrel. Of the 821 clopidogrel-treated patients, 277 had a $* 1 / * 17$ genotype, and 35 patients had a $* 17 / * 17$ genotype. The baseline characteristics for the 2 analyses can be found in Tables 1 and 2. All groups were well balanced in baseline characteristics, except for the more common use of proton pump inhibitors and oral anticoagulation in the clopidogrel-treated patients as compared to the ticagrelor- or prasugrel-treated patients. Tables I and II in the Data Supplement show what variables were used in univariate and multivariate regression analysis and what variables were used in the final model.

\section{Effect of the CYP2C19*17 allele in clopidogrel-treated patients}

For this analysis, 420 patients with a CYP2C19*1/*1 genotype and 312 patients with a $C Y P 2 C 19 * 1 / * 17$ or $* 17 / * 17$ genotype were available. An overview of the outcomes is presented in Table 3. There were no significant differences between the patients with CYP2C19*1/*1 genotype versus carriers of a *17 allele for the combined thrombotic outcome $3.8 \%$ versus $3.8 \%$, adjusted hazard ratio, 0.95 [95\% CI, 0.45-2.02], $\mathrm{P}=0.90$ Figure $2 \mathrm{~A})$ and the combined bleeding outcome $(11.2 \%$ versus $9.3 \%$, adjusted hazard ratio, 0.74 [95\% CI, 0.48-1.18], $\mathrm{P}=0.21$; Figure 2B).

\section{Clopidogrel in patients not carrying a $C Y P 2 C 19 * 2$ or $* 3$ allele versus} ticagrelor or prasugrel irrespective of CYP2C19 genotype For this analysis, 821 clopidogrel and 1608 ticagrelor or prasugrel-treated patients were available. Table 4 shows an overview of the outcomes. There were no significant differences in the combined thrombotic outcome $(3.4 \%$ versus $2.5 \%$, adjusted hazard ratio, 1.14 [95\% CI, 0.68-1.90], $\mathrm{P}=0.62$ Figure 3A), whereas the combined bleeding outcome occurred significantly less frequently in clopidogrel-treated patients $(9.9 \%$ versus $11.7 \%$, HR, 0.74 [95\% CI, 0.56-0.96], $\mathrm{P}=0.03$; Figure 3B).
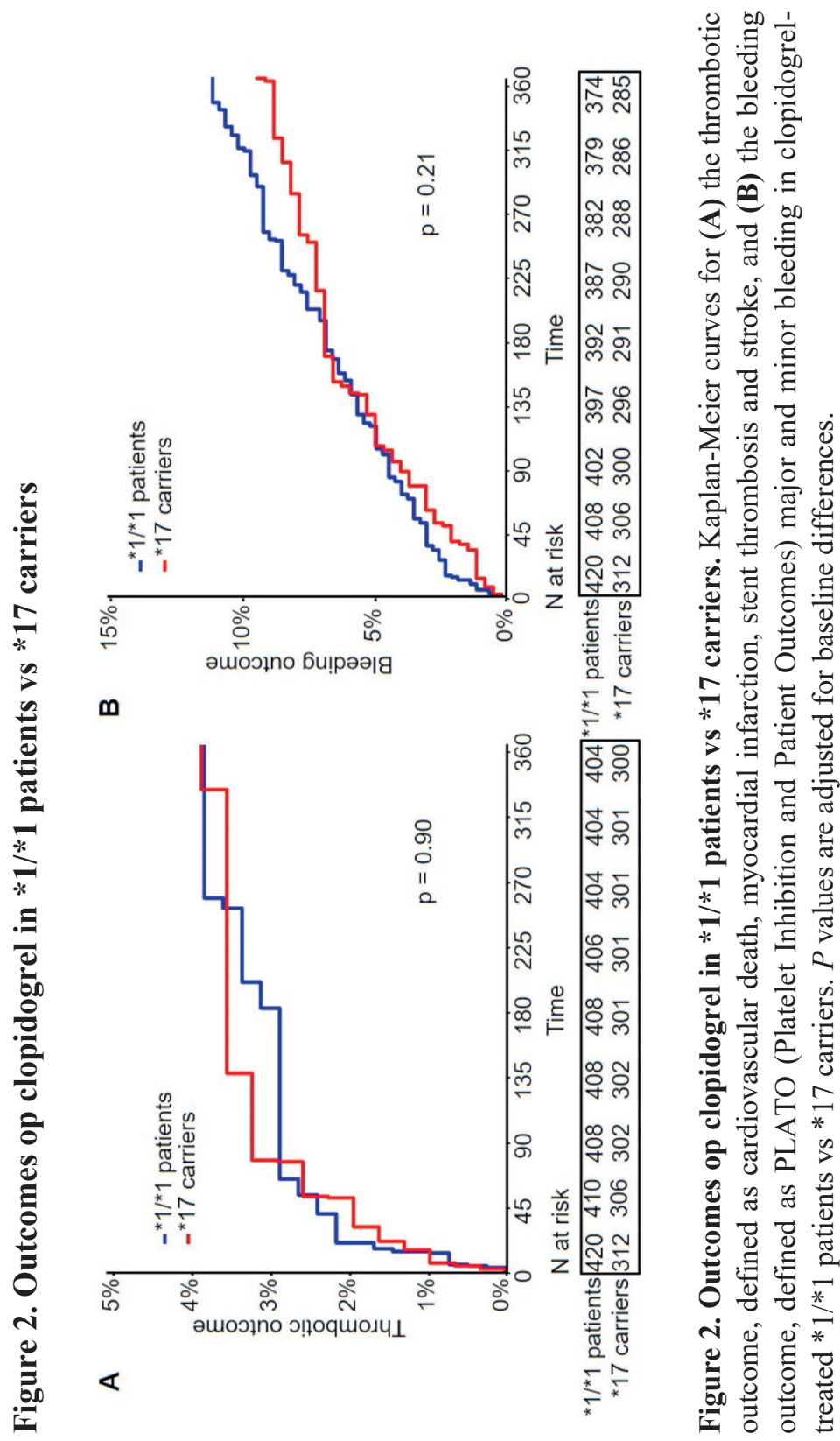


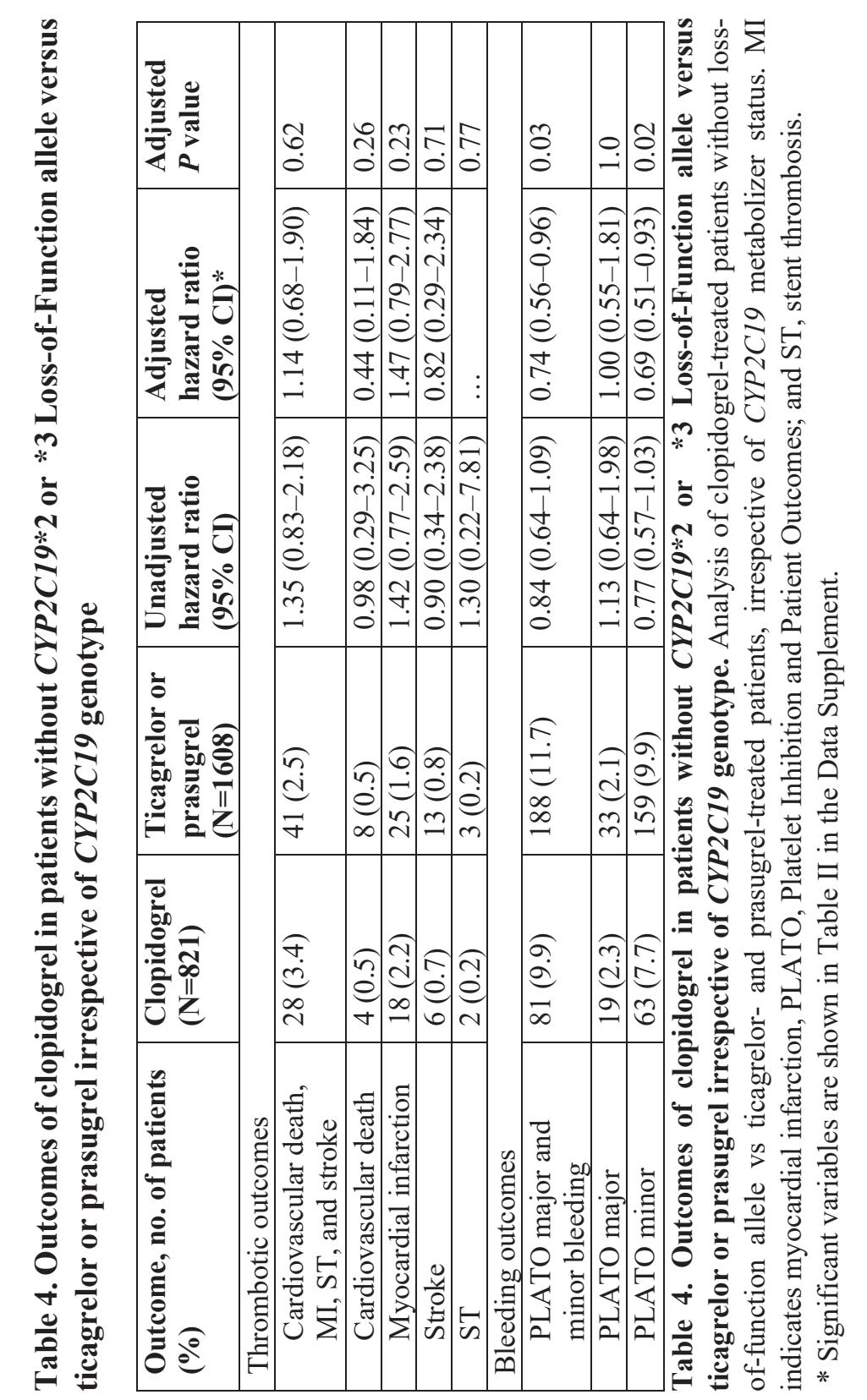

\section{Sensitivity Analyses}

Results for the two sensitivity analyses are presented in Table 5. The first analysis included 1550 patients without LoF allele, of which 821 patients were treated with clopidogrel and 729 with ticagrelor or prasugrel. The adjusted HR for the combined thrombotic outcome was 1.11 (95\% CI, 0.61-2.03, $\mathrm{P}=0.72$ ), whereas the adjusted HR for the combined bleeding outcome was $0.59(95 \%$ CI $, 0.44-0.80, \mathrm{P}<0.001)$. The on-treatment analysis included the 821 clopidogrel-treated patients and 1613 ticagreloror prasugrel-treated patients. The adjusted HR for the combined thrombotic outcome was 0.98 (95\% CI, $0.56-1.70, \mathrm{P}=0.94)$, whereas the adjusted HR for the combined bleeding outcome was 0.68 (95\% CI 0.52 $0.90, \mathrm{P}=0.008)$.

\section{Discussion}

In this prespecified subanalysis of the POPular Genetics trial, which included patients with ST-segment-elevation myocardial infarction who underwent primary PCI and compared clopidogrel-treated noncarriers of CYP2C19 LoF alleles with all ticagrelor- and prasugrel-treated patients, treatment with clopidogrel was associated with a significantly lower bleeding rate compared with treatment with ticagrelor or prasugrel. This finding held true in both the sensitivity analyses. The effect was primarily driven by a reduction of PLATO minor bleeding. There was no association found between clopidogrel use and a higher risk for the combined thrombotic outcome, although the number of patients with a recurrent myocardial infarction was numerically higher in clopidogrel-treated patients. These findings match the outcomes of the overall POPular Genetics trial [10]. Also in the PLATO genetic subanalysis, in which 3554 ticagrelor-treated patients without $C Y P 2 C 19 \mathrm{LoF}$ allele were compared with 3516 clopidogrel-treated patients without CYP2C19 LoF allele, similar to our second sensitivity analysis, the event rate for a combined thrombotic outcome consisting of cardiovascular death, myocardial infarction, and stroke was only numerically lower with the use of ticagrelor, not reaching statistical significance $(8.8 \%$ versus $10.0 \%$, HR, 0.86 [95\% CI, 0.74-1.01], $\mathrm{P}=0.06$ ) [11]. This in contrast to the overall PLATO trial, which showed clear benefit in reducing thrombotic risk with ticagrelor, compared with clopidogrel, at the cost of increased noncoronary artery bypass grafting surgery related major bleeding rates [3]. 

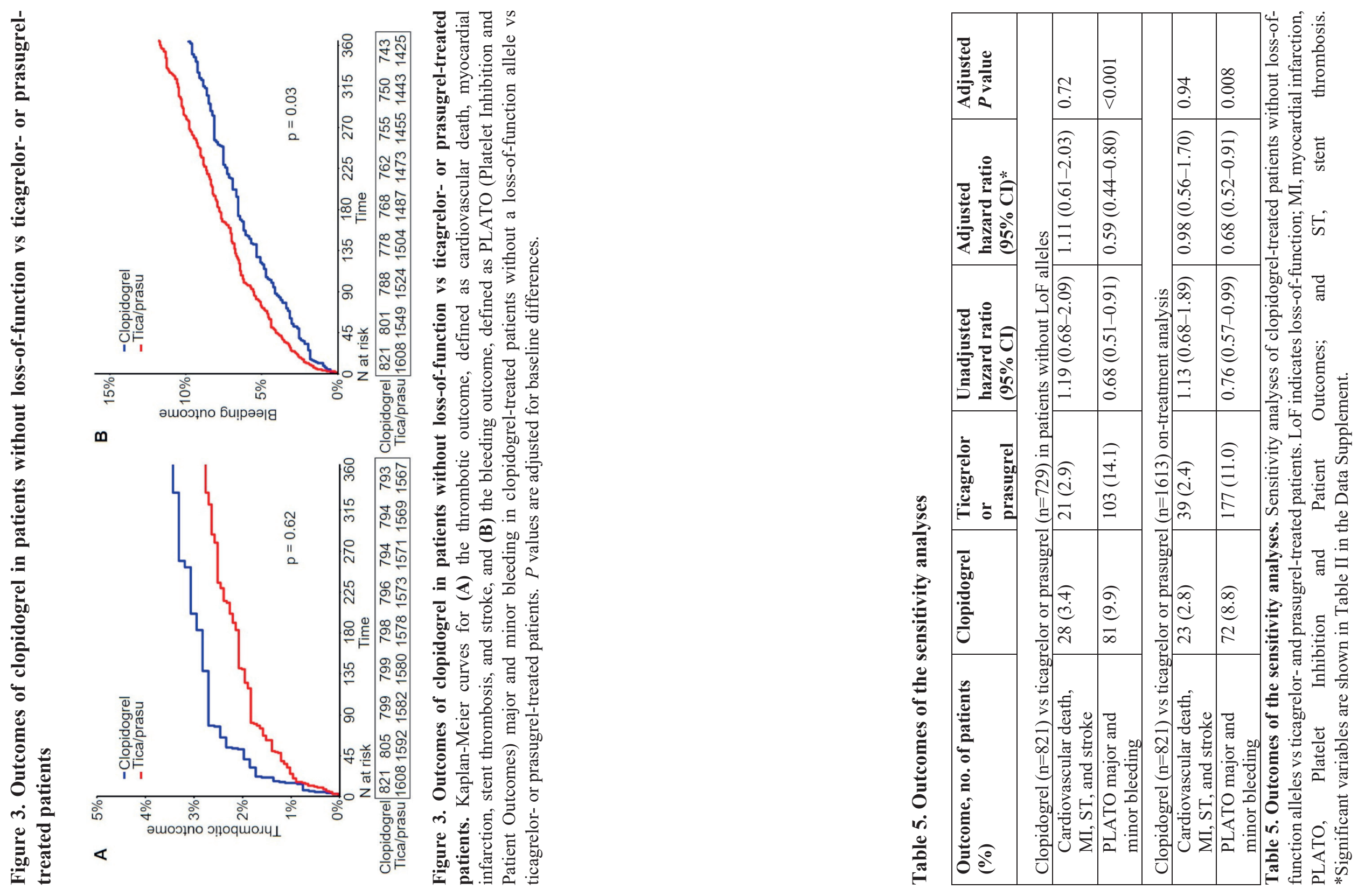
Nevertheless, the treatment effect in the PLATO subanalysis was almost similar to that of the main trial, so the lack of statistical significance could also have been due to a lack of power. With respect to the use of prasugrel, a genetic subanalysis of the TRITON-TIMI (Trial to Assess Improvement in Therapeutic Outcomes by Optimizing Platelet Inhibition With Prasugrel-Thrombolysis in Myocardial Infarction) 38 trial did not provide a direct comparison between prasugrel and clopidogrel in different CYP2C19 genetic subgroups but did conclude that clopidogrel-treated patients have significantly worse outcomes when carrying an LoF allele compared with noncarriers, which was not found in prasugrel-treated patients [15].

The primary analysis of the TAILOR-PCI trial (Tailored Antiplatelet Therapy Following PCI) included only patients who were carriers of $* 2$ or *3 LoF alleles [16]. It compared 903 patients in a CYP2C19 genotypeguided group, thus treated with ticagrelor, to 946 patients in a conventional therapy group, treated with clopidogrel, who were genotyped at the end of the follow-up period. The primary composite end point of cardiovascular death, myocardial infarction, stroke, stent thrombosis, and recurrent ischemia at 12 months, occurred less frequently in patients treated with ticagrelor but without reaching statistical significance (4\% versus $5.9 \%$, HR, 0.66 [95\% CI, 0.43-1.02], $\mathrm{P}=0.056$ ). Based on the results of these trials, one might conclude that in the absence of a CYP2C19 LoF allele, the balance in net clinical benefit ends up equal for clopidogrel versus ticagrelor, albeit with a somewhat lower bleeding risk with clopidogrel and somewhat lower thrombotic risk with ticagrelor. In clinical practice bleeding and thrombotic risk should be weighted to choose the optimal treatment strategy for the individual patient.

Furthermore, we studied the effect of the CYP2C19*17 gain-of-function polymorphism in clopidogrel-treated patients. This allele has been associated with higher enzyme activity, increased platelet inhibition, and an increased risk for bleeding, which was also found in the genetics subanalysis of the PLATO trial [11,17]. The evidence, however, is conflicting. A meta-analysis performed by Li et al. [8] containing data from 11 studies, found the CYP2C19*17 allele to be associated with less atherothrombotic events in patients treated with clopidogrel (based on six studies), whereas an increased risk for bleeding events was found (based on four studies). Comparable results were found in a meta-analysis performed by Zabalza et al. [18]. A meta-analysis performed by Bauer et al. [19], however, did not find any significant interaction for stent thrombosis or a composite of major adverse cardiac events related to the
CYP2C19*17 allele, although bleeding risk was not assessed. In a study performed by Lewis et al [20], CYP2C19*17 was in significant linkage disequilibrium with $C Y P 2 C 19^{*} 2$ and was not found to be an independent predictor for pharmacokinetic and pharmacodynamic outcome in patients treated with clopidogrel. The effect found for the $* 17$ allele was considered to be mostly, if not entirely, derived from non-independence with the CYP2C19*2 variant. Our data support this hypothesis, although no significant differences in thrombotic or bleeding outcomes were found according to CYP2C19*17 genotype. Therefore, our data do not support routine testing of $C Y P 2 C 19^{*} 17$ to guide antiplatelet therapy in clinical practice.

Our findings are clinically relevant for different reasons. Although $\mathrm{P} 2 \mathrm{Y}_{12}$ inhibitors are primarily initiated to reduce thrombotic risk, the inevitable bleeding risk associated with the use of those agents is clearly associated with morbidity and mortality [13]. Therefore, the use of a tailored antiplatelet strategy capable of balancing the bleeding and thrombotic risks is expected to improve overall clinical outcome. Although trials studying the use of platelet function testing in tailoring antiplatelet therapy failed to show superiority compared with standard treatment and is, therefore, not routinely recommended, our results add to the growing body of evidence that CYP2C19 genotype can be used to tailor antiplatelet therapy $[10,21,22]$. Recently published results from a large prospective multicentre study in France by Hulot et al. [23] also demonstrate that the availability of genetic results within days after ST-segment-elevation myocardial infarction can have a major impact on the prescription patterns of cardiologists. Additionally, because the use of ticagrelor and prasugrel is far more expensive compared with generic clopidogrel, a strategy in which two-thirds of patients can be treated with generic clopidogrel without losing the efficacy of the antiplatelet treatment is likely to be cost-effective [24]. A cost-effectiveness analysis was also one of the primary objectives of the POPular Genetics trial and is expected to be published in the near future. Finally, ticagrelor is associated with side effects like dyspnoea, which is a frequent reason to switch or even discontinue antiplatele therapy [25], whereas prasugrel needs a dose adjustment for the elderly and low body weight patients and is contraindicated in patients with previous stroke or transient ischemic attack [4].

Some limitations to our analysis need to be mentioned. First, it is a subanalysis of a larger trial and therefore not powered for its primary outcome. In particular, in the analysis of CYP 2 C19*17, the relatively low number of patients led us to the use of a dominant model (combining 
patients with $* 1 / * 17$ and $* 17 / * 17$ genotype) instead of a recessive mode (comparing $* 17$ homozygous patients to $* 1 / * 1$ and $* 1 / * 17$ patients). A possible effect limited to patients homozygous for the CYP2C19*17 allele might have been underestimated or missed. Second, we only tested for the $* 2$ and $* 3 \mathrm{LoF}$ alleles, since the prevalence of other LoF alleles is very small $(<1 \%$ for all LoF alleles combined). Therefore, a handful of clopidogrel-treated patients might now be identified as not carrying a LoF allele, although, in fact, they do. Third, due to the low number of patients treated with prasugrel, we cannot draw any conclusions for this drug specifically. Although the recently published ISAR-REACT 5 trial (Intracoronary Stenting and Antithrombotic Regimen: Rapid Early Action for Coronary Treatment 5) showed superiority of prasugrel over ticagrelor in patients with acute coronary syndrome, further research is needed to evaluate if our findings also apply to patients treated with prasugrel [26].

\section{Conclusion}

In patients after primary PCI not carrying a CYP2C19 LoF allele, the use of clopidogrel compared with ticagrelor or prasugrel was associated with lower bleeding rates, without significant increase in thrombotic events. No effect on clinical outcome was found for the CYP2C19*17 gain-offunction polymorphism.

\section{References}

1. Ibanez B, James S, Agewall S, et al. 2017 ESC guidelines for the management of acute myocardial infarction in patients presenting with ST-segment elevation. The Task Force for the management of acute myocardial infarction in patients presenting with ST-segment elevation of the European Society of Cardiology (ESC). Eur Heart J 2018; 39:119177

2. Levine GN, Bates ER, Bittl JA, et al. 2016 ACC/AHA guideline focused update on duration of dual antiplatelet therapy in patients with coronary artery disease: a report of the American College of Cardiology/American Heart Association Task Force on Clinical Practice Guidelines. Circulation 2016; 134:e123-e155

3. Wallentin L, Becker RC, Budaj A, et al. Ticagrelor versus clopidogrel in patients with acute coronary syndromes. N Engl J Med 2009; 361:10451057

4. Wiviott SD, Braunwald E, McCabe $\mathrm{CH}$, et al. Prasugrel versus clopidogrel in patients with acute coronary syndromes. N Engl J Med 2007; 357:2001-2015

5. Breet NJ, van Werkum JW, Bouman HJ, et al. Comparison of platelet function tests in predicting clinical outcome in patients undergoing coronary stent implantation. JAMA 2010; 303:754-762

6. Niu X, Mao L, Huang Y, et al. CYP2C19 polymorphism and clinical outcomes among patients of different races treated with clopidogrel: a systematic review and metaanalysis. J Huazhong Univ Sci Technolog Med Sci 2015; 35:147-156

7. Collet JP, Hulot JS, Pena A, et al. Cytochrome P450 2C19 polymorphism in young patients treated with clopidogrel after myocardial infarction: cohort study. Lancet 2009; 373:309-317

8. Li Y, Tang HL, Hu YF, et al. The gain-of-function variant allele CYP2C19*17: a double-edged sword between thrombosis and bleeding in clopidogrel-treated patients. J Thromb Haemost 2012; 10:199-206

9. Moon JY, Franchi F, Rollini F, et al. Role of genetic testing in patients undergoing percutaneous coronary intervention. Expert Rev Clin Pharmacol 2018; 11:151-164

10. Claassens DMF, Vos GJA, Bergmeijer TO, et al. A genotype-guided strategy for oral P2Y12 inhibitors in primary PCI. N Engl J Med 2019; 381:1621-1631

11. Wallentin L, James S, Storey RF, et al. Effect of CYP2C19 and ABCB single nucleotide polymorphisms on outcomes of treatment with ticagrelor versus clopidogrel for acute coronary syndromes: a genetic substudy of the PLATO trial. Lancet 2010; 376:1320-1328 
12. Puymirat E, Cayla G, Cottin Y, et al. Twenty-year trends in profile, management and outcomes of patients with ST-segment elevation myocardial infarction according to use of reperfusion therapy: data from the FASTMI program 1995-2015. Am Heart J 2019; 214:97-106

13. Généreux P, Giustino G, Witzenbichler B, et al. Incidence, predictors, and impact of post-discharge bleeding after percutaneous coronary intervention. J Am Coll Cardiol 2015; 66:1036-1045

14. Bergmeijer TO, Janssen PW, Schipper JC, et al. CYP2C19 genotypeguided antiplatelet therapy in ST-segment elevation myocardial infarction patients-Rationale and design of the Patient Outcome after primary PCI (POPular) Genetics study. Am Heart J 2014; 168:16-22.e1

15. Mega JL, Close SL, Wiviott SD, et al. Genetic variants in ABCB1 and CYP2C19 and cardiovascular outcomes after treatment with clopidogrel and prasugrel in the TRITON-TIMI 38 trial: a pharmacogenetic analysis Lancet 2010; 376:1312-1319

16. Pereira NL, Farkouh ME, So D, et al. Effect of genotype-guided oral P2Y12 inhibitor selection vs conventional clopidogrel therapy on ischemic outcomes after percutaneous coronary intervention: the TAILOR-PCI randomized clinical trial. JAMA 2020; 324:761-771

17. Sibbing D, Koch W, Gebhard D, et al. Cytochrome $2 \mathrm{C} 19 * 17$ allelic variant, platelet aggregation, bleeding events, and stent thrombosis in clopidogrel-treated patients with coronary stent placement. Circulation 2010; 121:512-518

18. Zabalza M, Subirana I, Sala J, et al. Meta-analyses of the association between cytochrome CYP2C19 loss- and gain-of-function polymorphisms and cardiovascular outcomes in patients with coronary artery disease treated with clopidogrel. Heart 2012; 98:100-108

19. Bauer T, Bouman HJ, van Werkum JW, et al. Impact of CYP2C19 variant genotypes on clinical efficacy of antiplatelet treatment with clopidogrel: systematic review and meta-analysis. BMJ 2011; 343:d4588

20. Lewis JP, Stephens SH, Horenstein RB, et al. The CYP2C19*17 variant is not independently associated with clopidogrel response. J Thromb Haemost 2013; 11:1640-1646

21. Collet JP, Cuisset T, Rangé G, et al. Bedside monitoring to adjust antiplatelet therapy for coronary stenting. N Engl J Med 2012;367:21002109

22. Sibbing D, Aradi D, Jacobshagen C, et al. Guided de-escalation of antiplatelet treatment in patients with acute coronary syndrome undergoing percutaneous coronary intervention (TROPICAL-ACS): a randomised, open-label, multicentre trial. Lancet 2017; 390:1747-1757

23. Hulot JS, Chevalier B, Belle L, et al. Routine CYP2C19 genotyping to adjust thienopyridine treatment after primary PCI for STEMI: results of the GIANT Study. JACC Cardiovasc Interv 2020; 13:621-630
24. Wang Y, Yan BP, Liew D, et al. Cost-effectiveness of cytochrome P450 $2 \mathrm{C} 19 * 2$ genotype-guided selection of clopidogrel or ticagrelor in Chinese patients with acute coronary syndrome. Pharmacogenomics J 2018; $18: 113-120$

25. Bergmeijer TO, Janssen PWA, van Oevelen M, et al. Incidence and causes for early ticagrelor discontinuation: a "real-world" dutch registry experience. Cardiology 2017; 138:164-168

26. Schüpke S, Neumann FJ, Menichelli M, et al. Ticagrelor or prasugrel in patients with acute coronary syndromes. N Engl J Med 2019; 381:1524 1534 
Supplementary appendix 
Table I. Variables used in the CYPC19*17 univariate and multivariable regression analysis

\begin{tabular}{|c|c|c|}
\hline Outcome & $\begin{array}{l}\text { Significant variables } \\
\text { in univariate model }\end{array}$ & $\begin{array}{l}\text { Significant variables } \\
\text { in multivariable model }\end{array}$ \\
\hline \multicolumn{3}{|l|}{ Thrombotic outcomes } \\
\hline $\begin{array}{l}\text { Combined thrombotic } \\
\text { outcome } \\
\text { (Cardiovascular death, } \\
\text { MI, definite ST, stroke) }\end{array}$ & Age & - \\
\hline $\begin{array}{l}\text { Death from vascular } \\
\text { causes }\end{array}$ & - & - \\
\hline Myocardial Infarction & - & - \\
\hline Stroke & - & - \\
\hline $\begin{array}{l}\text { Definite Stent } \\
\text { thrombosis }\end{array}$ & - & - \\
\hline \multicolumn{3}{|l|}{ Bleeding outcomes } \\
\hline $\begin{array}{l}\text { PLATO major \& minor } \\
\text { bleeding }\end{array}$ & Age & - \\
\hline PLATO major & Age & - \\
\hline PLATO minor & Age & - \\
\hline
\end{tabular}

Table I: Variables used in univariate and multivariable regression for clopidogrel treated patients stratified according to CYP2C19*17 carrier status*.

MI: Myocardial infarction, PLATO: platelet inhibition and patient outcome, ST: stent thrombosis.

* Age and smoker were selected for the model.
Table II. Variables used in the CYP2C19 LoF noncarrier univariate and multivariable regression analysis.

\begin{tabular}{|c|c|c|}
\hline Outcome & $\begin{array}{l}\text { Significant variables } \\
\text { in univariate model }\end{array}$ & $\begin{array}{l}\text { Significant variables } \\
\text { in multivariable model }\end{array}$ \\
\hline \multicolumn{3}{|l|}{ Thrombotic outcomes } \\
\hline $\begin{array}{l}\text { Combined thrombotic } \\
\text { outcome } \\
\text { (Cardiovascular death, } \\
\text { MI, definite ST, stroke) }\end{array}$ & $\begin{array}{l}\text { Aspirin, vitamin K } \\
\text { antagonist, NOAC at } \\
\text { discharge }\end{array}$ & $\begin{array}{l}\text { Vitamin } \mathrm{K} \text { antagonist, } \\
\text { NOAC at discharge }\end{array}$ \\
\hline $\begin{array}{l}\text { Death from vascular } \\
\text { causes }\end{array}$ & $\begin{array}{l}\text { Aspirin, vitamin } \mathrm{K} \\
\text { antagonist, NOAC at } \\
\text { discharge }\end{array}$ & $\begin{array}{l}\text { Vitamin } \mathrm{K} \text { antagonist, } \\
\text { NOAC at discharge }\end{array}$ \\
\hline Myocardial Infarction & $\begin{array}{l}\text { Stent length, NOAC at } \\
\text { discharge }\end{array}$ & $\begin{array}{l}\text { Stent length, NOAC at } \\
\text { discharge }\end{array}$ \\
\hline Stroke & $\begin{array}{l}\text { Aspirin, vitamin } \mathrm{K} \\
\text { antagonist, NOAC at } \\
\text { discharge }\end{array}$ & $\begin{array}{l}\text { Vitamin } \mathrm{K} \text { antagonist, } \\
\text { NOAC at discharge }\end{array}$ \\
\hline $\begin{array}{l}\text { Definite Stent } \\
\text { thrombosis }\end{array}$ & - & - \\
\hline \multicolumn{3}{|l|}{ Bleeding outcomes } \\
\hline $\begin{array}{l}\text { PLATO major \& minor } \\
\text { bleeding }\end{array}$ & $\begin{array}{l}\text { Sex, vitamin K } \\
\text { antagonist, NOAC at } \\
\text { discharge }\end{array}$ & $\begin{array}{l}\text { Sex, vitamin K } \\
\text { antagonist, NOAC at } \\
\text { discharge }\end{array}$ \\
\hline PLATO major & $\begin{array}{l}\text { Aspirin, vitamin K } \\
\text { antagonist, NOAC at } \\
\text { discharge }\end{array}$ & $\begin{array}{l}\text { Vitamin K antagonist, } \\
\text { NOAC at discharge }\end{array}$ \\
\hline PLATO minor & $\begin{array}{l}\text { Sex, aspirin, vitamin K } \\
\text { antagonist, NOAC at } \\
\text { discharge }\end{array}$ & $\begin{array}{l}\text { Vitamin } \mathrm{K} \text { antagonist, } \\
\text { NOAC at discharge }\end{array}$ \\
\hline
\end{tabular}

Table II: Variables used in univariate and multivariable regression analysis for clopidogrel in patients without $C Y P 2 C 19 * 2$ or *3 LoF allele versus ticagrelor or prasugrel in all patients (irrespective of CYP2C19 genotype)*.

LoF: loss-of-function, MI: Myocardial infarction, NOAC: Novel anticoagulant, PLATO: platelet inhibition and patient outcome, PPI: proton pump inhibitor, ST: Stent thrombosis.

* Sex, aspirin, PPI, vitamin K antagonist and NOAC at discharge were selected for the model. 
Clopidogrel in noncarriers of CYP2C19 loss-c of-function alleles versus ticagrelor in elderly patients with acute coronary syndrome:

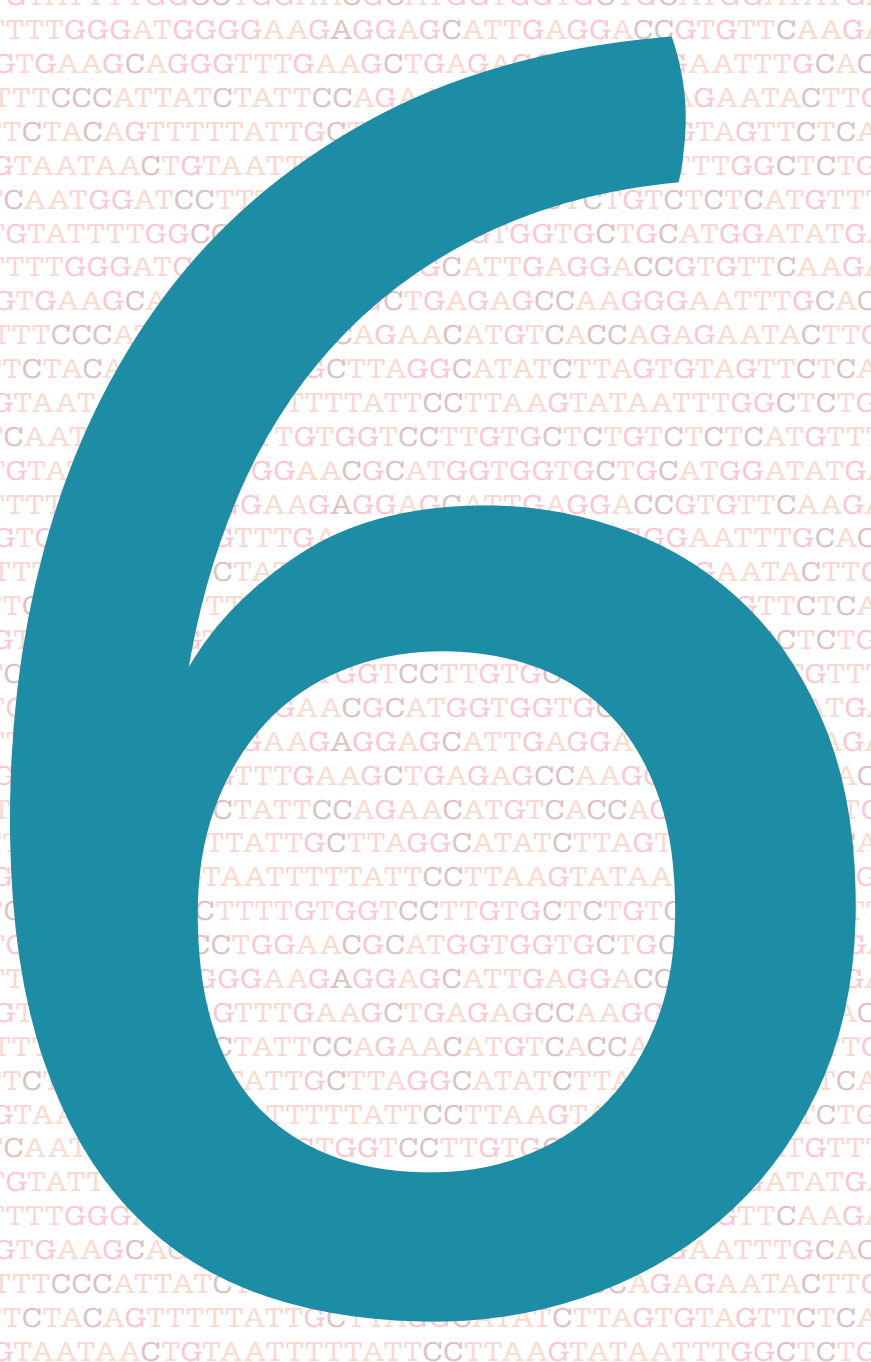
A pre-specified sub analysis from the POPular Genetics and POPular Age trials

Daniel M.F. Claassens, Marieke E. Gimbel, Thomas 0. Bergmeijer, Gerrit J.A. Vos, Renicus S. Hermanides, Pim van der Harst, Emanuele Barbato, Carmine Morisco, Richard M. Tjon Joe Gin, Evelyn A. de Vrey, Ton A.C.M. Heestermans, J. Wouter Jukema, Clemens von Birgelen, Reinier A. Waalewijn, Sjoerd H. Hofma, Frank R. den Hartog, Michiel Voskuil, Arnoud W.J. van 't Hof, Folkert W. Asselbergs, A. Mosterd, Jean-Paul R. Herrman, Willem J.M. Dewilde, Bakhtawar K. Mahmoodi, Vera H.M. Deneer, Jurriën M. ten Berg

International Journal of Cardiology 2021; 334:10-17

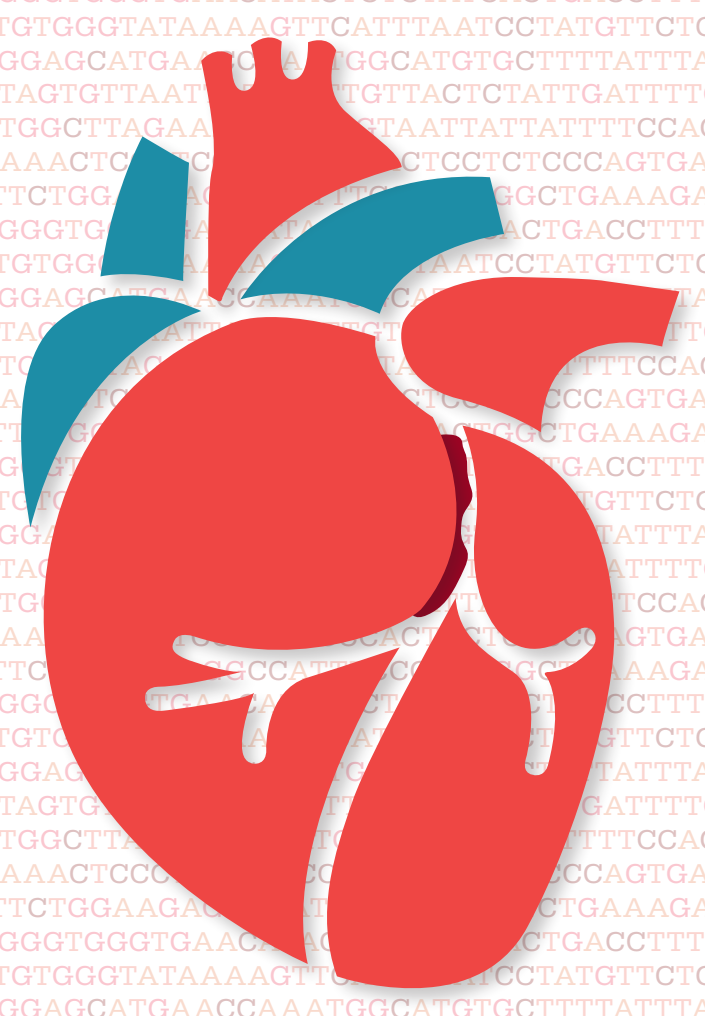




\section{Abstract}

Background

Patients with acute coronary syndrome (ACS) who are carrying CYP2C19 loss-of-function alleles derive less benefit from clopidogrel treatment. Despite this, in elderly patients, clopidogrel might be preferred over more potent $\mathrm{P}_{2} \mathrm{Y}_{12}$ inhibitors due to a lower bleeding risk. Whether CYP2C19 genotype-guided antiplatelet treatment in the elderly could be of benefit has not been studied specifically.

\section{Methods}

Patients aged 70 years and older with known CYP2C19*2 and *3 genotype were identified from the POPular Genetics and POPular Age trials. Noncarriers of loss-of-function alleles treated with clopidogrel were compared to patients, irrespective of CYP2C19 genotype, treated with ticagrelor and to clopidogrel treated carriers of loss-of-function alleles. We assessed net clinical benefit (all-cause death, myocardial infarction, stroke and Platelet Inhibition and Patient Outcomes (PLATO) major bleeding), atherothrombotic outcomes (cardiovascular death, myocardial infarction, stroke) and bleeding outcomes (PLATO major and minor bleeding).

\section{Results}

A total of 991 patients were assessed. There was no significant difference in net clinical benefit ( $17.2 \%$ vs. $15.1 \%$, adjusted hazard ratio (adjHR) $1.05,95 \%$ confidence interval (CI) $0.77-1.44$ ), atherothrombotic outcomes $(9.7 \%$ vs. $9.2 \%$, adjHR $1.00,95 \%$ CI $0.66-1.50)$, and bleeding outcomes $(17.7 \%$ vs. $19.8 \%$, adjHR $0.80,95 \%$ CI $0.62-1.12)$ between clopidogrel in noncarriers of loss-of-function alleles and ticagrelor respectively.

\section{Conclusion}

In ACS patients aged 70 years and older, there was no significant difference in net clinical benefit and atherothrombotic outcomes between noncarriers of a loss-of-function allele treated with clopidogrel and patients treated with ticagrelor. The bleeding rate was numerically; though not statistically significant, lower in patients using clopidogrel.

\section{Introduction}

In patients with acute coronary syndrome (ACS), dual antiplatelet therapy (DAPT) plays an essential role in preventing recurrent atherothrombotic events [1]. Although clopidogrel is still widely used in combination with aspirin, The Platelet Inhibition and Patient Outcomes (PLATO) [2] and Trial to Assess Improvement in Therapeutic Outcomes by Optimizing Platelet Inhibition with Prasugrel Thrombolysis in Myocardial Infarction (TRITON TIMI) 38 [3] trials demonstrated superiority of ticagrelor and prasugrel, respectively, over clopidogrel in preventing atherothrombotic events in patients with ACS. Therefore, current guidelines favour these more potent platelet inhibitors over clopidogrel $[4,5]$. However, patients treated with ticagrelor or prasugrel are at a higher bleeding risk compared to patients treated with clopidogrel [2,3], which is even more pronounced in elderly patients [6]. The Clopidogrel versus Ticagrelor or Prasugrel in Patients Aged 70 years or older with non-ST-elevation Acute Coronary Syndrome (POPular Age) trial showed that in elderly patients, DAPT with clopidogrel has similar results as DAPT with the more potent platelet inhibitors in terms of ischemic events, while bleeding events were lower [7].

Clopidogrel is a prodrug, which is converted to its active metabolite by CYP P450 hepatic enzymes [8]. Approximately 30\% of the Caucasian population show an inadequate response to clopidogrel when measured with platelet function tests, which is associated with a worse clinical outcome [9]. At least part of this variation in drug efficacy can be explained by genetic variations, of which the CYP2C19*2 and *3 loss-of-function alleles are the most important [10]. For ticagrelor and prasugrel, no relevant gene-drug interaction have been found [11,12]. Although the correlation of CYP2C19 genotype and clinical outcome was not assessed in the POPular Age trial so far, it can be hypothesised that also in elderly patients CYP2C19 genotype influences the balance between benefit and harm when the more potent $\mathrm{P} 2 \mathrm{Y}_{12}$ inhibitors are prescribed in comparison to clopidogrel.

A strategy, in which the antiplatelet treatment was chosen based on CYP2C19 genotype, although in a cohort of patients with ST-segment elevation myocardial infarction (STEMI), was evaluated in the GenotypeGuided Antiplatelet Therapy in ST-Segment Elevation Myocardia Infarction Patients - Patient Outcome after Primary Percutaneous Coronary Intervention (POPular Genetics) trial [13]. In this trial, a genotype-guided strategy was found to be non-inferior compared to standard treatment with ticagrelor or prasugrel for a net clinical benefit 
outcome and ischemic events, while bleeding events were lower. While both atherothrombotic and bleeding event rates are higher in elderly patients, and bleeding events have shown to be strongly correlated to a worse clinical outcome [14], the use of genetic testing may improve clinical decision-making in selecting patients who benefit most from clopidogrel versus prasugrel or ticagrelor treatment.

In the present analysis, containing ACS patients aged 70 years and older derived from the POPular Age and POPular Genetics trial cohorts, we compare the use of clopidogrel in noncarriers of CYP2C19 loss-of-function alleles with ticagrelor, irrespective of $C Y P 2 C 19$ genotype and we assess the effect of CYP2C19 loss-of-function alleles in clopidogrel treated elderly patients.

\section{Methods}

\section{Study design}

The detailed design and results of the POPular Age and POPular Genetics trials have been published previously $[15,16]$. In brief, the POPular Genetics trial was an open label, assessor blinded, randomized controlled trial performed in 10 centres in the Netherlands, Belgium and Italy. Between 2012 and 2018, 2,488 patients with STEMI undergoing primary PCI aged 21 years and older were included. Patients were randomized within 48 hours of primary PCI to either a standard treatment arm (treatment with ticagrelor or prasugrel for one year), or to the genotypeguided arm (treatment adjustment after rapid $C Y P 2 C 19$ genetic testing). In the genotype-guided arm, patients carrying a CYP2C19*2 or *3 loss-offunction allele were treated with ticagrelor or prasugrel, while noncarriers $(* 1 / * 1)$ were treated with clopidogrel. The POPular Age trial was an open label, assessor blinded, randomized controlled trial performed in 12 centres in the Netherlands. Between 2013 and 2018, 1,002 patients with non-STsegment elevation myocardial infarction (NSTEMI) and unstable angina aged 70 years and older were included. Within 72 hours after hospita admission, patients were randomized to either treatment with clopidogrel, or to treatment with ticagrelor or prasugrel. In both trials, follow-up duration was 12 months. An institutional review board at all study sites approved the trials and all patients provided written informed consent.

In both trials, blood samples were collected for genotyping purposes, although only the samples derived from patients in the genotyping arm of the POPular Genetics trial were genotyped prospectively. All other samples were genotyped after study completion for the CYP2C19*2 and *3 loss-of-function alleles. In the POPular Age trial, blood samples were not collected in all participating hospitals and are therefore not available for all patients. Genotyping was performed by LGC Biosearch Technologies (Hoddesdon, United Kingdom) using a KASP genotyping assay.

\section{Statistical analysis}

The current analysis was pre-specified in both trials, although this subanalysis was not prospectively powered. Therefore, the number of patient is based on the available patients of 70 years and older in whom CYP2C19 genotype was available in the study cohorts. The following outcome parameters were assessed: (1) a net clinical benefit outcome, consisting of all-cause death, myocardial infarction, stroke and PLATO major bleeding; (2) an atherothrombotic outcome, consisting of cardiovascular death, myocardial infarction and stroke; and (3) a bleeding outcome, consisting of PLATO major and minor bleeding. Other outcomes were the individual components of the composite outcomes. The outcome definitions were identical to the definitions used in both main trials, in which a blinded event committee adjudicated all adverse clinical events.

The current analysis compared noncarriers of the CYP2C19*2 and *3 alleles treated with clopidogrel to patients treated with ticagrelor. A smal number of prasugrel treated patients was therefore excluded. A modified intention-to-treat analysis was performed in which patients were divided in a clopidogrel or a ticagrelor treated group, based on the drug that was prescribed at discharge after the index hospital admission. Two additiona analyses were performed. An on-treatment analysis, in which patients were censored if they discontinued or switched $\mathrm{P} 2 \mathrm{Y}_{12}$ inhibitor therapy and an analysis in which patients treated with oral anticoagulation were excluded. Additionally, outcomes in clopidogrel treated patients were assessed according to CYP2C19 loss-of-function allele carrier status and we assessed outcomes in the subgroups of the POPular Age and POPular Genetics trial separately.

Variables are presented as number (percentages) and mean \pm standard deviation, or median with an interquartile range. Missing data was not imputed. Time-to-event curves were constructed using the Kaplan-Meier method. Hazard ratios (HR) with 95\% confidence intervals (CI) were calculated using Cox proportional-hazard models. To adjust for possible confounders, all baseline characteristics with a p-value $<0.10$ were selected for univariate regression analysis. If there was a significant interaction $(\mathrm{p}<0.05)$ in the univariate analysis, they were selected for 


\section{Figure 1. Flowchart}

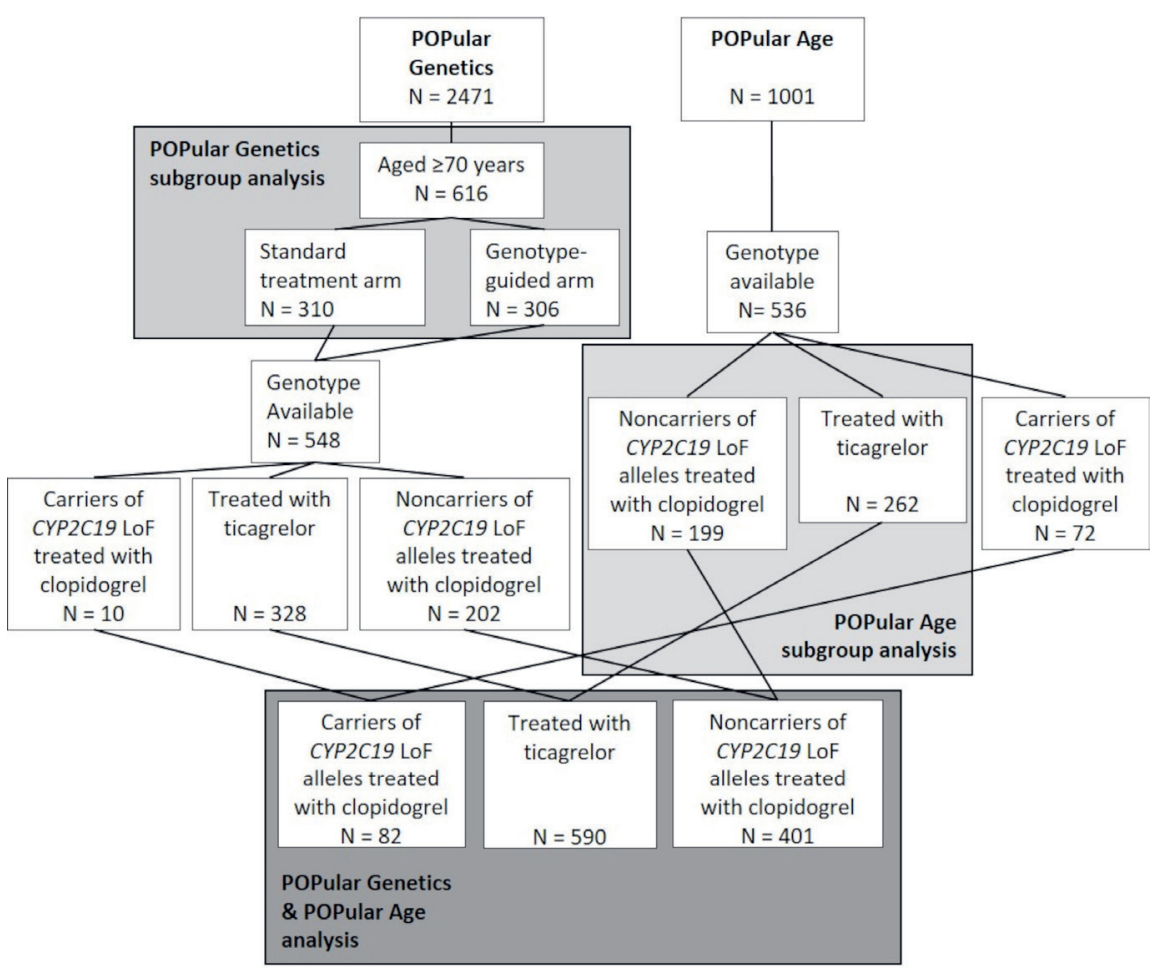

Figure 1. Flowchart of patient selection for the different subgroup analyses. LoF:

Loss-of-function multivariable regression analysis. The final model included only those characteristics with a significant interaction in the multivariable analysis. P-values $<0.05$ were considered statistically significant. Data were analysed using ' $\mathrm{R}$ ' (version 3.6.0 or later).

\section{Results}

Figure 1 shows how the different subgroups were selected from the two trials. For 1,084 patients aged 70 years and older (548 patients from the POPular Genetics trial and 536 patients from the POPular Age trial) the CYP2C19 genotype was available. Of those, 401 were noncarriers of lossof-function alleles treated with clopidogrel, 82 were carriers of loss-offunction alleles treated with clopidogrel and 590 were treated with ticagrelor. Baseline characteristics are presented in Table 1. The mean age was 77 years. In noncarriers of loss-of-function alleles treated with clopidogrel, more female patients and a lower baseline haemoglobin level were observed, compared to patients treated with ticagrelor. Almost 10\% of the elderly patients in this study received medical treatment only, without coronary angiography, even though it was suspected they had obstructive coronary artery disease. In $11 \%$ of patients who underwent coronary angiography, coronary artery disease was found, but there was no clear target lesion, while another $10 \%$ of patients who underwent coronary angiography, but not a PCI, either received coronary artery bypass surgery or they were deemed unsuitable for PCI, (e.g. due to the location or type of lesion and/or due to comorbidities). The sensitivity analysis excluding patients with oral anticoagulation included 345 clopidogrel and 530 ticagrelor treated patients. Baseline differences were similar to that of the main analysis. Between clopidogrel treated carriers and noncarriers of lossof-function alleles there were many different baseline characteristics, like higher rates of dyslipidaemia, prior PCI and prior stroke, in addition to the more frequent diagnosis of NSTEMI and unstable angina at discharge in the $C Y P 2 C 19$ loss-of-function allele carrier group and the less frequent use of drug eluting stents and PCI in this group.

In the POPular Genetics subgroup, 616 patients aged 70 years and older were available for analysis. Of those, 306 patients were in the genotypeguided cohort and 310 patients were in the standard treatment arm. Both groups were well balanced with regards to baseline and procedural characteristics. In the POPular Age subgroup, CYP2C19 genotype was available in 536 patients. Of those, 199 patients were noncarriers of a CYP2C19 loss-of-function allele treated with clopidogrel, and 265 patients were treated with ticagrelor. Groups were well balanced, except for more peripheral arterial disease in the ticagrelor treated patients. 


\section{Table 1. Baseline characteristics}

\begin{tabular}{|c|c|c|c|c|c|}
\hline Characteristics & $\begin{array}{l}\text { Clopidogrel } \\
\text { noncarrier } \\
\mathbf{N}=401\end{array}$ & $\begin{array}{l}\text { Ticagrelor } \\
\mathbf{N}=\mathbf{5 9 0}\end{array}$ & $\begin{array}{l}P \\
\text { value }\end{array}$ & $\begin{array}{l}\text { Clopidogrel } \\
\text { LoF allele } \\
\text { carriers } \\
\mathrm{N}=\mathbf{8 2} \\
\end{array}$ & $\begin{array}{l}P \\
\text { value }\end{array}$ \\
\hline $\begin{array}{l}\text { Age (years, mean } \\
\pm \text { SD) }\end{array}$ & $77.0 \pm 5.4$ & $77.0 \pm 5.4$ & 0.84 & $78.0 \pm 5.1$ & 0.09 \\
\hline Female sex & $161(40.1)$ & $189(32.0)$ & 0.01 & $32(39.0)$ & 0.95 \\
\hline $\begin{array}{l}\mathrm{BMI}(\mathrm{kg} / \mathrm{m} 2 \\
\text { mean } \pm \mathrm{SD})\end{array}$ & $26.3 \pm 3.98$ & $26.5 \pm 3.77$ & 0.54 & $26.4 \pm 3.55$ & 0.87 \\
\hline $\begin{array}{l}\text { Creatinine } \\
\text { clearance (eGFR } \\
\text { CKD-EPI) } \\
<60 \mathrm{ml} / \text { minute } 1.7 \\
3 \mathrm{~m} 2\end{array}$ & $127(31.8)$ & $165(28.2)$ & 0.25 & $24(29.3)$ & 0.76 \\
\hline $\begin{array}{l}\text { Hemoglobin at } \\
\text { admission } \\
(\mathrm{mmol} / 1 \text {, mean } \pm \\
\text { SD) }\end{array}$ & $8.40 \pm 0.83)$ & $8.54 \pm 0.92)$ & 0.02 & $8.51 \pm 0.98$ & 0.34 \\
\hline \multicolumn{6}{|l|}{ Medical history } \\
\hline Current smoker & $64(16.1)$ & $112(19.2)$ & 0.24 & $10(12.3)$ & 0.50 \\
\hline Hypertension & $250(62.3)$ & $357(60.5)$ & 0.61 & $56(69.1)$ & 0.30 \\
\hline Dyslipidemia & $143(35.8)$ & $234(39.8)$ & 0.22 & $41(50.0)$ & 0.02 \\
\hline Diabetes Mellitus & $81(20.2)$ & $128(21.7)$ & 0.63 & $25(30.5)$ & 0.06 \\
\hline $\begin{array}{l}\text { Myocardial } \\
\text { infarction }\end{array}$ & $73(18.2)$ & $107(18.2)$ & 1.0 & $21(25.6)$ & 0.16 \\
\hline PCI & $52(13.0)$ & $95(16.1)$ & 0.22 & $19(23.2)$ & 0.03 \\
\hline CABG & $40(10.0)$ & $56(9.5)$ & 0.89 & $13(15.9)$ & 0.17 \\
\hline Stroke & $10(2.5)$ & $20(3.4)$ & 0.54 & $7(8.5)$ & 0.02 \\
\hline $\begin{array}{l}\text { Peripheral arterial } \\
\text { disease }\end{array}$ & $27(6.8)$ & $54(9.2)$ & 0.21 & $8(9.8)$ & 0.47 \\
\hline Bleeding & $16(4.0)$ & $20(3.4)$ & 0.74 & $5(6.1)$ & 0.38 \\
\hline \multicolumn{6}{|c|}{ CYP2C19 genotype } \\
\hline $\begin{array}{l}\text { Normal } \\
\text { metabolizer }\end{array}$ & $402(100)$ & $375(63.6)$ & & $0(0)$ & \\
\hline $\begin{array}{l}\text { Intermediate } \\
\text { metabolizer }\end{array}$ & $0(0)$ & $189(32.0)$ & & 75 (91.5) & \\
\hline Poor metabolizer & $0(0)$ & $17(2.9)$ & & $7(8.5)$ & \\
\hline \multicolumn{6}{|l|}{ At discharge } \\
\hline Diagnosis & & & 0.13 & & $\begin{array}{l}<0.00 \\
1\end{array}$ \\
\hline Unstable angina & $17(4.3)$ & $22(3.8)$ & & $9(11.1)$ & \\
\hline NSTEMI & $177(44.4)$ & $225(38.4)$ & & 56 (69.1) & \\
\hline
\end{tabular}

\begin{tabular}{|l|l|l|l|l|l|}
\hline STEMI & $202(50.6)$ & $328(56.0)$ & & $10(12.3)$ & \\
\hline Aspirin & $349(88.4)$ & $529(90.7)$ & 0.27 & $70(86.4)$ & 0.76 \\
\hline P2Y 12 inhibitor & & & & & \\
\hline Clopidogrel & $383(100)$ & $0(0)$ & & $69(100)$ & \\
\hline Ticagrelor & $0(0)$ & $554(100)$ & & $0(0)$ & \\
\hline $\begin{array}{l}\text { Oral } \\
\text { anticoagulation }\end{array}$ & $56(14.0)$ & $60(10.2)$ & 0.09 & $14(17.1)$ & 0.58 \\
\hline Beta blocker & $304(77.0)$ & $451(77.4)$ & 0.95 & $62(76.5)$ & 1.00 \\
\hline Ace inhibitor & $241(61.0)$ & $372(63.8)$ & 0.41 & $52(64.2)$ & 0.68 \\
\hline ARB & $73(18.5)$ & $109(18.7)$ & 1.0 & $13(16.0)$ & 0.72 \\
\hline Statin & $375(93.8)$ & $553(94.2)$ & 0.87 & $72(87.8)$ & 0.10 \\
\hline Procedural characteristics & & & & \\
\hline CAG performed & $365(91.0)$ & $557(94.4)$ & 0.17 & $71(86.6)$ & 0.35 \\
\hline $\begin{array}{l}\text { Femoral access } \\
\text { site }\end{array}$ & $125(34.2)$ & $184(33.0)$ & \multicolumn{2}{|l|}{$29(40.8)$} & \\
\hline $\begin{array}{l}\text { Radial access } \\
\text { site }\end{array}$ & $240(65.8)$ & $366(65.7)$ & \multicolumn{2}{|l|}{$42(59.2)$} & \\
\hline $\begin{array}{l}\text { Multivessel } \\
\text { disease }\end{array}$ & $213(53.2)$ & $330(56.1)$ & 0.41 & $41(50.0)$ & 0.68 \\
\hline POBA & $10(2.5)$ & $9(1.5)$ & 0.39 & $2(2.4)$ & 1.00 \\
\hline Bare metal stent & $12(3.0)$ & $20(3.4)$ & 0.87 & $0(0)$ & 0.23 \\
\hline Drug eluting stent & $267(66.6)$ & $419(71.0)$ & 0.16 & $44(53.7)$ & 0.04 \\
\hline
\end{tabular}

Table 1. Baseline characteristics of patients aged 70 years and older from both the POPular Age and POPular Genetics trial with acute coronary syndrome in whom the CYP2C19 genotype was known, stratified according to $\mathrm{P}_{2} \mathrm{Y}_{12}$ inhibitor treatment and LoF carrier status.

All variables were expressed as number (\%), unless specified ACS: Acute coronary syndrome, ARB: Angiotensin II receptor blocker, BMI: Body mass index, CABG: Coronary artery bypass surgery, CAG: Coronary angiography, LoF: Loss-of-function, NSTEMI: Non-ST elevation myocardial infarction, PCI: Percutaneous coronary intervention, POBA: Plain old balloon angioplasty, STEMI: ST-elevation myocardial infarction. 

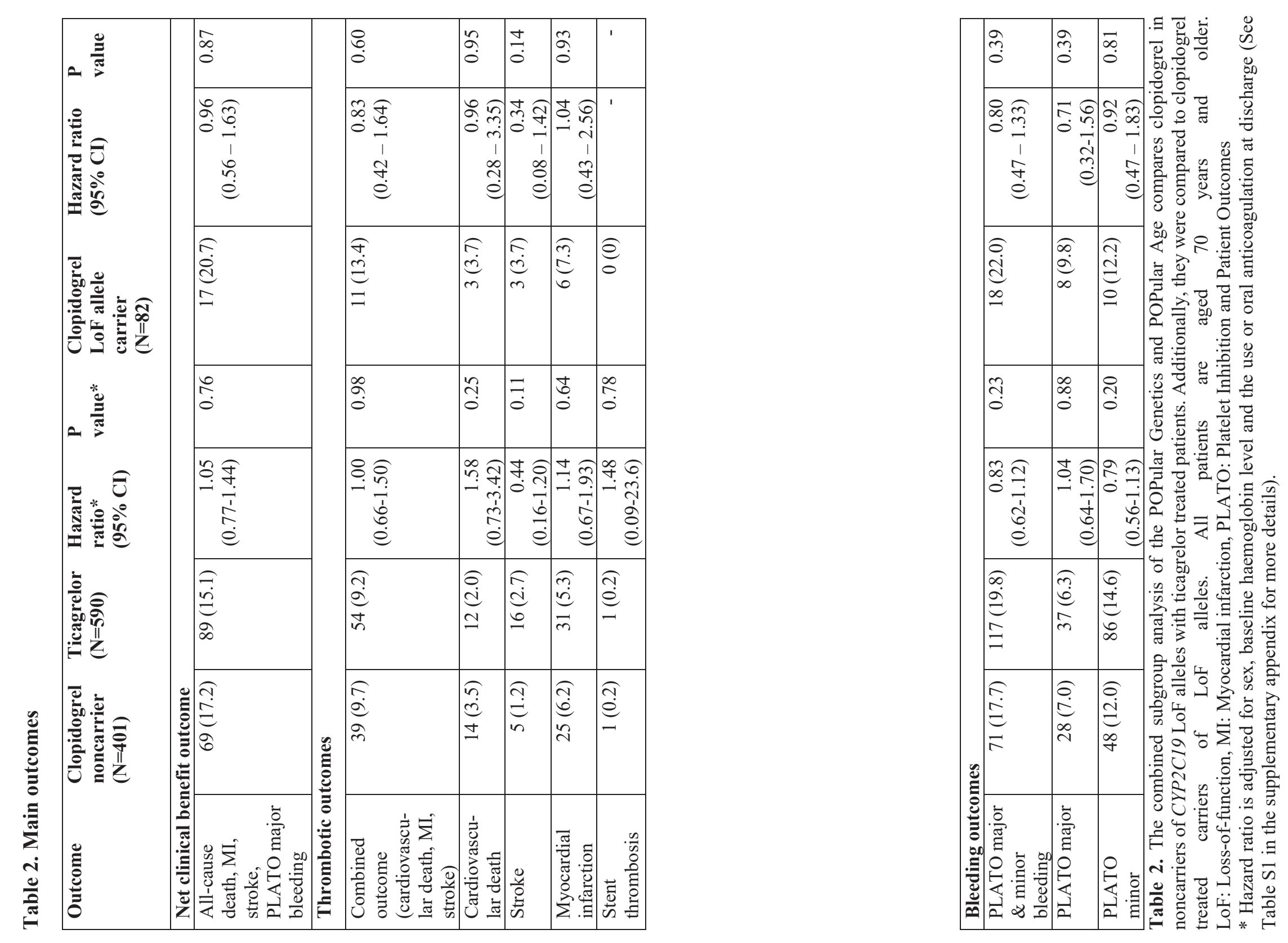

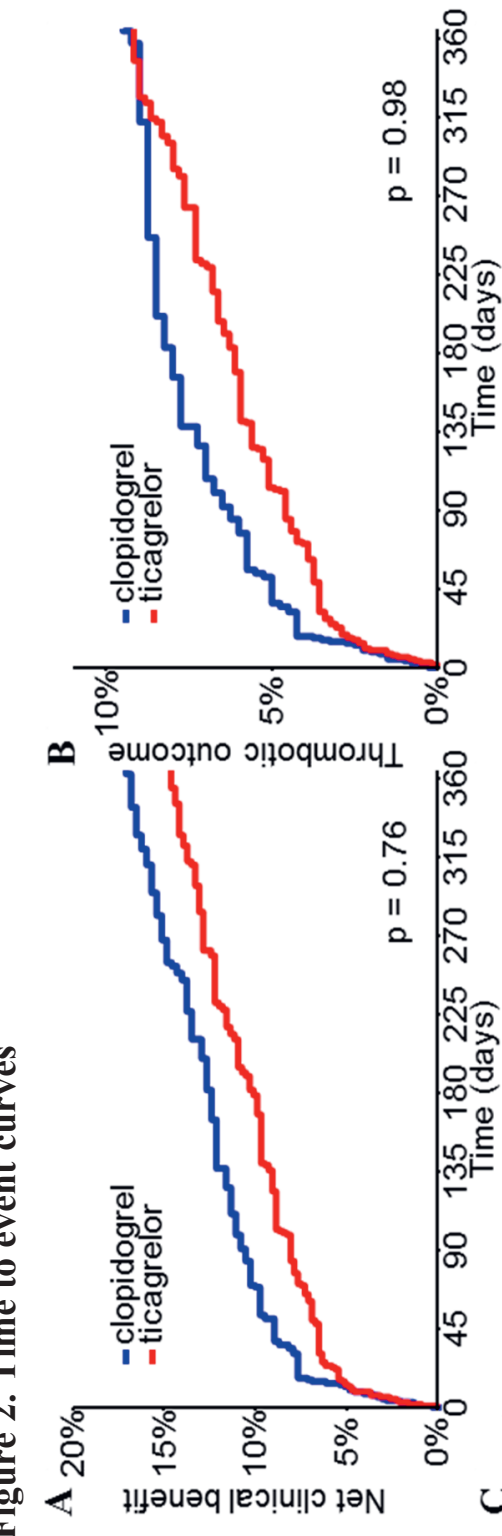

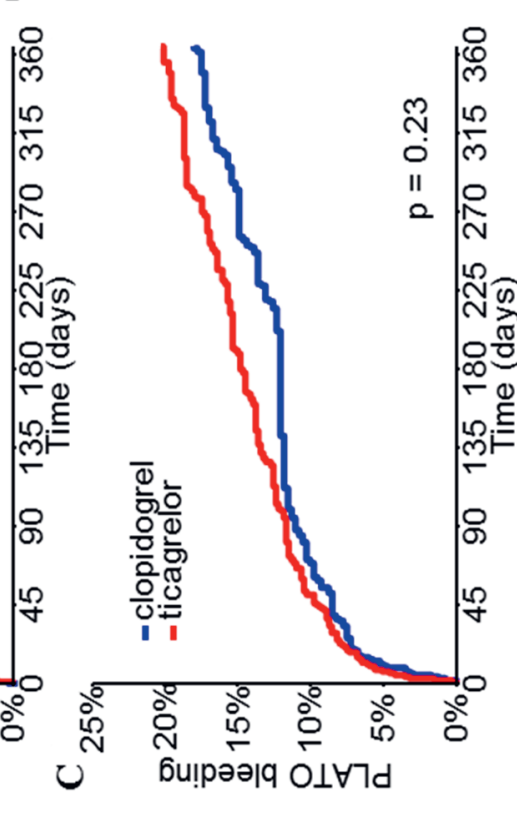

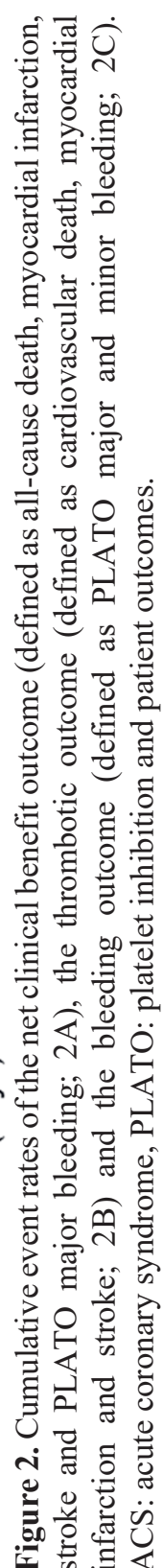

Results are presented in Table 2, Figure 2 and Figure 3. The variables included in the multivariable regression analysis to adjust for baseline differences are presented in Table S1 in the supplementary appendix. After adjustment for differences in baseline characteristics between the groups, there was no statistically significant difference in the net clinical benefit outcome (17.2\% vs. $15.1 \%$, adjusted hazard ratio (adjHR) $1.05,95 \% \mathrm{CI}$, $0.77-1.44, p=0.76)$ (Figure $2 \mathrm{~A}$ ), thrombotic outcome $(9.7 \%$ vs. $9.2 \%$, adjHR 1.00, 95\%CI, $0.66-1.50, \mathrm{p}=0.98$ ) (Figure 2B), and bleeding outcome $(17.7 \%$ vs. $19.8 \%$, adjHR $0.83,95 \% \mathrm{CI}, 0.62-1.12, \mathrm{p}=0.23)$ (Figure 2C), in noncarriers of loss-of-function alleles treated with clopidogrel compared to patients treated with ticagrelor irrespective of CYP2C19 genotype. Results for the on-treatment analysis and the analysis excluding patients treated with oral anticoagulation are presented in Figure 3 and in Table S2 in the supplementary appendix.

There were no significant differences between the clopidogrel treated noncarriers group and the small clopidogrel treated carriers of loss-offunction alleles group (Table 2 ) in the net clinical benefit outcome $(17.2 \%$ vs. $20.7 \%$, HR $0.96,95 \% \mathrm{CI}, 0.56-1.63, \mathrm{p}=0.87)$, thrombotic outcome (9.7\% vs. $13.4 \%$, HR $0.83,95 \%$ CI, $0.42-1.64, \mathrm{p}=0.60)$ and bleeding outcome (17.7\% vs. $22.0 \%$, HR $0.80,95 \%$ CI $0.47-1.33, \mathrm{p}=0.39)$.

Results for the subgroup analysis of the POPular Age and POPular Genetics trial cohorts are presented in Figure 3 and in Table S3 in the supplementary appendix. In both analyses, there are no significant differences in any of the outcomes.

\section{Discussion}

In this pre-specified sub analysis from the POPular Genetics and POPular Age trials, we compared the use of clopidogrel in noncarriers of CYP2C19 loss-of-function alleles with the use of ticagrelor in patients with ACS aged 70 years and older. No statistically significant differences were found for both atherothrombotic and bleeding outcomes. Nevertheless, the finding of a comparable atherothrombotic event rate (adjHR 1.00) combined with a numerically, though not statistically significant, lower number of bleeding events in the clopidogrel treated patients without CYP2C19 loss-offunction allele (adjHR 0.83) is clinically relevant. Additionally, we identified numerically less 


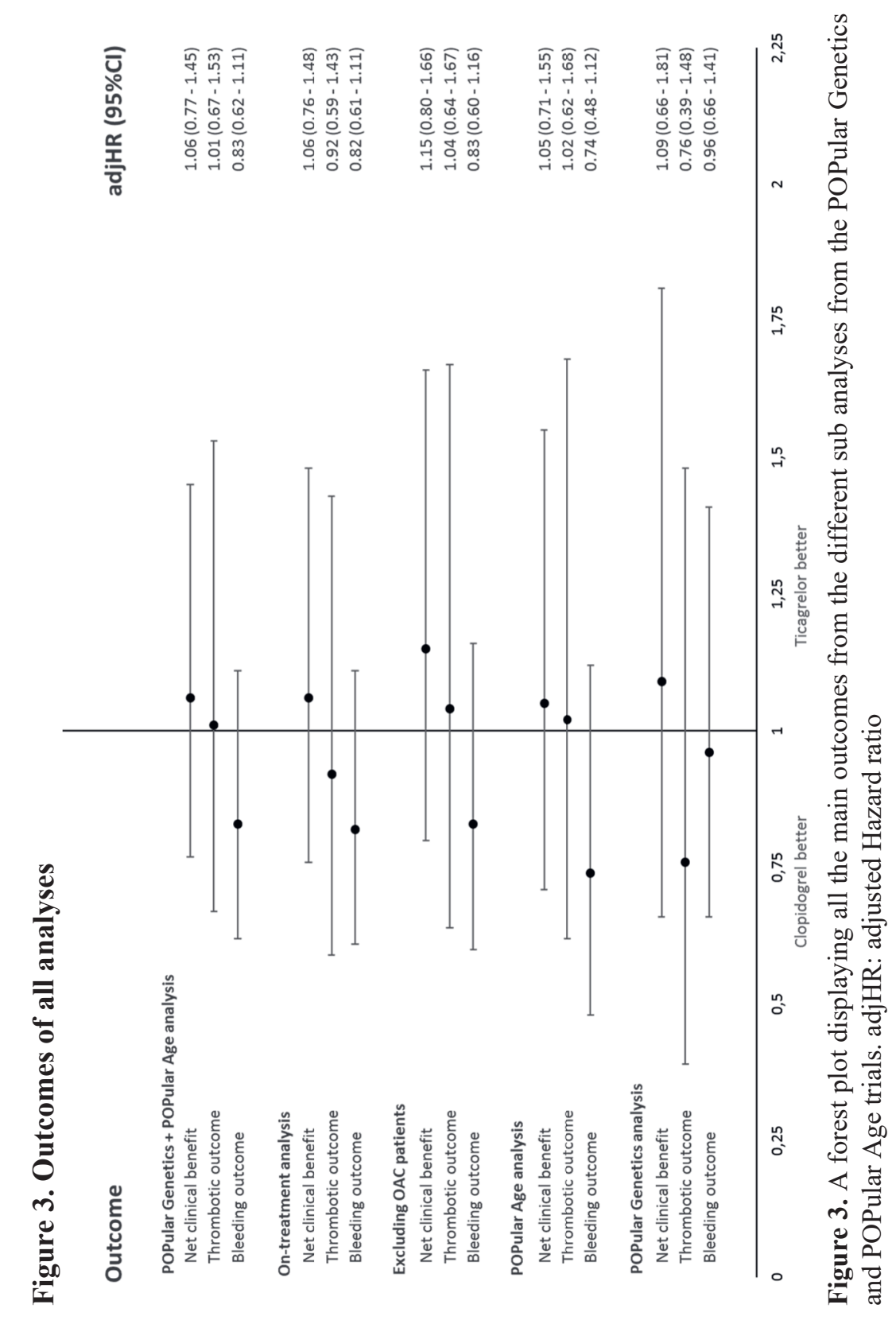

atherothrombotic events and bleeding events in noncarriers of CYP2C19 loss-of-function alleles treated with clopidogrel compared to carriers of loss-of-function alleles, though, in this very small subgroup with many baseline differences, these differences were not statistically significant. Therefore, any real conclusions cannot be made based on this data.

These results are in accordance with the elderly subgroup analysis of the TRITON-TIMI 38 trial $(\mathrm{n}=1809$ ), comparing prasugrel with clopidogrel, which found a lower bleeding risk in clopidogrel-treated patients of 75 years and older [3]. In contrast, the subgroup analysis of the PLATO trial, studying the use of ticagrelor in the elderly population $(\mathrm{n}=2878)$, showed a benefit of ticagrelor in reducing ischemic events, without significantly increasing the risk of bleeding, although fatal bleeding and non-CABG related major bleeding were higher in the elderly treated with ticagrelor [17]. These results were confirmed in the Bremen STEMI registry, which included 1087 STEMI patients aged 75 years older treated with primary PCI, comparing ticagrelor to clopidogrel [18]. This benefit with ticagrelor was not found in the POPular Age trial $(\mathrm{n}=1002)$, studying patients of 70 years or older with non-ST elevation ACS [7]. While thrombotic risk was not statistically different with the use of ticagrelor or clopidogrel, the bleeding rate was lower in the clopidogrel treated patients. These results are strengthened by the large $(\mathrm{n}=14,005)$, nationwide SWEDEHEART registry including patients of 80 years and older with ACS [19]. Although the evidence is conflicting - which for some part might be due to differences in patient selection, selection of different age groups and development and improvement of drug-eluting stents, enhanced secondary prevention and improved intervention techniques in the past decade decreasing ischemic risk - it might be concluded that the more potent $\mathrm{P} 2 \mathrm{Y}_{12}$ inhibitors are not superior for all elderly patients with ACS regarding net clinical benefit outcomes, and clopidogrel is a reasonable alternative.

The POPular Genetics trial found lower bleeding rates without increasing atherothrombotic rates when using $C Y P 2 C 19$ genetic testing compared to standard treatment with ticagrelor. These results also applied to the elderly subgroup, where no significant interaction was found. The results from the combined POPular Genetics and POPular Age subgroup analysis confirm the main results of the POPular Genetics trial in this elderly subgroup, with similar atherothrombotic and lower bleeding event rates in the clopidogrel group compared to the ticagrelor group. This was not statistically significant due to the limited sample size of this subgroup. The TAILOR PCI trial also investigated CYP2C19 genetic testing and included patients who underwent PCI $(82 \%$ because of ACS, $18 \%$ with stable coronary 
artery disease) and randomized them to the control arm or genotype-guided therapy arm. In the control arm patients received clopidogrel, and in the genotype-guided arm patients received clopidogrel if they were noncarriers of the $C Y P 2 C 19 * 2$ and $* 3$ loss-of-function alleles or ticagrelor if they were carriers of these alleles [20]. A numeric reduction was found in thrombotic events in carriers of CYP2C19 loss-of-function alleles when treated with ticagrelor as compared to clopidogrel, though this difference was not statistically significant $(p=0.056)$, which is in agreement with the results of this subanalysis [20]. However, there was also no significant benefit of the genotype-guided strategy in regard to the safety outcome, probably because they compared with clopidogrel in the control arm instead of ticagrelor. The elderly subgroup analysis of patients aged 75 years and older of the TAILOR PCI trial $(\mathrm{n}=261)$ showed no significant interaction [20]. Combining the results of the TAILOR PCI with this subgroup analysis, specifying antiplatelet therapy by means of $C Y P 2 C 19$ genotyping might be promising for improving clinical outcomes in the elderly ACS patient, however further research is needed.

To this day, clopidogrel remains the most widely used platelet inhibitor in elderly patients with ACS [21-23]. Although most evidence regarding elderly patients is based on subgroup analysis and is underpowered, it might be concluded that the more potent $\mathrm{P} 2 \mathrm{Y}_{12}$ inhibitors prasugrel and ticagrelor are not superior in all elderly patients with ACS regarding net clinical benefit outcomes, and clopidogrel could be a reasonable alternative. This effect might be the strongest in patients without CYP2C19 loss-of-function allele, who are expected to have optimal clopidogrel efficacy. A CYP2C19 genotype guided strategy might be promising for improving clinical outcome in the elderly ACS patient.

Several limitations to this study need to be mentioned. Although this is one of the largest analyses comparing clopidogrel with ticagrelor in elderly patients with ACS and known CYP2C19 genotype, it was not powered to find statistical differences between both treatment strategies. In addition, both trials had an open-label design and the POPular Age trial was not designed to compare a genotype-guided strategy with standard treatment. Therefore, our results are hypothesis-generating and should be interpreted with caution. Furthermore, since our main analysis only included clopidogrel treated noncarriers of loss-of-function alleles, results cannot be extrapolated to all clopidogrel-treated patients. Lastly, since we compared our data to ticagrelor-treated patients only, it is unknown how outcomes compare to prasugrel-treated patients.

\section{Conclusion}

In patients with ACS aged 70 years and older, there were no significant differences in atherothrombotic or bleeding event rates between noncarriers of a CYP2C19 loss-of-function allele treated with clopidogrel, and patients treated with ticagrelor irrespective of CYP2C19 genotype. Nevertheless, the bleeding rate was numerically, though not statistically significant, lower in clopidogrel treated patients. Further research focusing on the value of CYP2C19 genotyping in this specific and vulnerable group of patients might help to find an optimal antiplatelet treatment for the elderly. 


\section{References}

1. Yusuf S, Zhao F, Mehta SR, et al. Effects of Clopidogrel in Addition to Aspirin in Patients with Acute Coronary Syndromes without ST-Segment Elevation. N Engl J Med 2001; 345:494-502

2. Wallentin L, Becker RC, Budaj A, et al. Ticagrelor versus clopidogrel in patients with acute coronary syndromes. N Engl J Med 2009; 361:10451057

3. Wiviott SD, Braunwald E, McCabe $\mathrm{CH}$, et al. Prasugrel versus clopidogrel in patients with acute coronary syndromes. N Engl J Med 2007; 357:2001-2015

4. Collet JP, Thiele H, Barbato E, et al. 2020 ESC Guidelines for the management of acute coronary syndromes in patients presenting without persistent ST-segment elevation. Eur Heart J 2021; 42:1289-1367

5. Valgimigli M, Bueno H, Byrne RA, et al. 2017 ESC focused update on dual antiplatelet therapy in coronary artery disease developed in collaboration with EACTS. Eur Heart J 2018; 39:213-260

6. Yeh RW, Secemsky EA, Kereiakes DJ, et al. Development and Validation of a Prediction Rule for Benefit and Harm of Dual Antiplatelet Therapy Beyond 1 Year After Percutaneous Coronary Intervention. JAMA 2016; 315:1735-1749

7. Gimbel M, Qaderdan K, Willemsen L, et al. Clopidogrel versus ticagrelor or prasugrel in patients aged 70 years or older with non-ST-elevation acute coronary syndrome (POPular AGE): the randomised, open-label, non-inferiority trial. Lancet 2020; 395:1374-1381

8. Savi P, Pereillo JM, Uzabiaga MF, et al. Identification and biologica activity of the active metabolite of clopidogrel. Thromb Haemost 2000 84:891-896

9. Breet NJ, Van Werkum JW, Bouman HJ, et al. Comparison of platelet function tests in predicting clinical outcome in patients undergoing coronary stent implantation. JAMA 2010; 303:754-762

10. Harmsze AM, Van Werkum JW, Ten Berg JM, et al. CYP2C19*2 and CYP2C9*3 alleles are associated with stent thrombosis: A case-control study. Eur Heart J 2010; 31:3046-3053

11. Wallentin L, James S, Storey RF, et al. Effect of CYP2C19 and ABCB1 single nucleotide polymorphisms on outcomes of treatment with ticagrelor versus clopidogrel for acute coronary syndromes: A genetic substudy of the PLATO trial. Lancet 2010; 376:1320-1328

12. Mega JL, Close SL, Wiviott SD, et al. Genetic variants in ABCB1 and CYP2C19 and cardiovascular outcomes after treatment with clopidogre and prasugrel in the TRITON-TIMI 38 trial: A pharmacogenetic analysis. Lancet 2010; 376:1312-1319
13. Claassens DMF, Vos GJA, Bergmeijer TO, et al. A Genotype-Guided Strategy for Oral P2Y 12 Inhibitors in Primary PCI. N Engl J Med 2019 381:1621-1631

14. Eikelboom JW, Mehta SR, Anand SS, et al. Adverse Impact of Bleeding on Prognosis in Patients With Acute Coronary Syndromes. Circulation 2006; 114:774-782

15. Bergmeijer TO, Janssen PWA, Schipper JC, et al. CYP2C19 genotypeguided antiplatelet therapy in ST-segment elevation myocardial infarction patients - Rationale and design of the Patient Outcome after primary PCI (POPular) Genetics study. Am Heart J 2014; 168:16-22.e1

16. Qaderdan K, Ishak M, Heestermans AACM, et al. Ticagrelor or prasugre versus clopidogrel in elderly patients with an acute coronary syndrome Optimization of antiplatelet treatment in patients 70years and olderrationale and design of the POPular AGE study. Am Heart J 2015 170:981-985.e1

17. Husted S, James S, Becker RC, et al. Ticagrelor Versus Clopidogrel in Elderly Patients With Acute Coronary Syndromes. Circ Cardiovasc Qual Outcomes 2012; 5:680-688

18. Schmucker J, Fach A, Mata Marin LA, et al. Efficacy and Safety of Ticagrelor in Comparison to Clopidogrel in Elderly Patients With STSegment-Elevation Myocardial Infarctions. J Am Heart Assoc 2019; 8: 012530

19. Szummer K, Montez-Rath ME, Alfredsson J, et al. Comparison between Ticagrelor and Clopidogrel in Elderly Patients with an Acute Coronary Syndrome: Insights from the SWEDEHEART Registry. Circulation 2020; 142:1700-1708

20. Pereira NL, Farkouh ME, So D, et al. Effect of Genotype-Guided Oral P2Y12 Inhibitor Selection vs Conventional Clopidogrel Therapy on Ischemic Outcomes after Percutaneous Coronary Intervention: The TAILOR-PCI Randomized Clinical Trial. JAMA 2020; 324:761-771

21. Yudi MB, Clark DJ, Farouque O, et al. Clopidogrel, prasugrel or ticagrelor in patients with acute coronary syndromes undergoing percutaneous coronary intervention. Intern Med J 2016; 46:559-565

22. Sheikh Rezaei S, Geroldinger A, Heinze G, et al. Clopidogrel, prasugrel, or ticagrelor use and clinical outcome in patients with acute coronary syndrome: A nationwide long-term registry analysis from 2009 to 2014 Int J Cardiol 2017; 235:61-66

23. Baber U, Sartori S, Aquino M, et al. Use of prasugrel vs clopidogrel and outcomes in patients with acute coronary syndrome undergoing percutaneous coronary intervention in contemporary clinical practice: Results from the PROMETHEUS study. Am Heart J 2017; 188:73-81 
Supplementary appendix 
Table S1. Variables selected for regression analysis

\begin{tabular}{|c|c|c|c|}
\hline Outcome & $\begin{array}{l}\text { Variables } \\
\text { tested in } \\
\text { univariate } \\
\text { regression }\end{array}$ & $\begin{array}{l}\text { Significant } \\
\text { variables in } \\
\text { univariate } \\
\text { regression }\end{array}$ & $\begin{array}{l}\text { Significant } \\
\text { variables in } \\
\text { multivariable } \\
\text { regression model }\end{array}$ \\
\hline & \multicolumn{3}{|c|}{ Net clinical benefit outcome } \\
\hline \multirow[t]{2}{*}{$\begin{array}{l}\text { All-cause death, MI, } \\
\text { stroke, PLATO } \\
\text { major bleeding }\end{array}$} & $\begin{array}{r}\text { Female sex, } \\
\text { Hb, OAC }\end{array}$ & $\mathrm{Hb}, \mathrm{OAC}$ & $\mathrm{Hb}, \mathrm{OAC}$ \\
\hline & \multicolumn{3}{|c|}{ Thrombotic outcomes } \\
\hline $\begin{array}{l}\text { Combined Outcome } \\
\text { (Cardiovascular } \\
\text { death, MI, stroke) }\end{array}$ & $\begin{array}{r}\text { Female sex, } \\
\text { Hb, OAC }\end{array}$ & $\mathrm{Hb}, \mathrm{OAC}$ & $\mathrm{Hb}, \mathrm{OAC}$ \\
\hline $\begin{array}{l}\text { Cardiovascular } \\
\text { death }\end{array}$ & $\begin{array}{r}\text { Female sex, } \\
\text { Hb, OAC }\end{array}$ & $\mathrm{Hb}, \mathrm{OAC}$ & $\mathrm{Hb}, \mathrm{OAC}$ \\
\hline $\begin{array}{l}\text { Myocardial } \\
\text { infarction }\end{array}$ & $\begin{array}{r}\text { Female sex, } \\
\text { Hb, OAC }\end{array}$ & $\mathrm{Hb}, \mathrm{OAC}$ & $\mathrm{Hb}, \mathrm{OAC}$ \\
\hline Stroke & $\begin{array}{r}\text { Female sex, } \\
\mathrm{Hb}, \mathrm{OAC}\end{array}$ & OAC & $\mathrm{OAC}$ \\
\hline \multirow[t]{2}{*}{ Stent thrombosis } & $\begin{array}{r}\text { Female sex, } \\
\mathrm{Hb}, \mathrm{OAC}\end{array}$ & - & \\
\hline & \multicolumn{3}{|c|}{ Bleeding outcomes } \\
\hline $\begin{array}{l}\text { PLATO major \& } \\
\text { minor bleeding }\end{array}$ & $\begin{array}{r}\text { Female sex, } \\
\mathrm{Hb}, \mathrm{OAC}\end{array}$ & $\mathrm{Hb}, \mathrm{OAC}$ & $\mathrm{Hb}, \mathrm{OAC}$ \\
\hline PLATO major & $\begin{array}{r}\text { Female sex, } \\
\text { Hb, OAC }\end{array}$ & $\mathrm{Hb}, \mathrm{OAC}$ & $\mathrm{Hb}, \mathrm{OAC}$ \\
\hline PLATO minor & $\begin{array}{r}\text { Female sex, } \\
\text { Hb, OAC }\end{array}$ & OAC & $\mathrm{OAC}$ \\
\hline
\end{tabular}

Table S1. Female sex, haemoglobin level at admission and the use of oral anticoagulation at discharge were selected for univariate and multivariable regression. In univariate regression, female sex was not a significant interaction variable for any of the outcomes. Hb: Haemoglobin, MI: Myocardial infarction, OAC: Oral anticoagulation, PLATO: Platelet Inhibition and Patient Outcomes

\begin{tabular}{|c|c|c|c|c|c|c|}
\hline 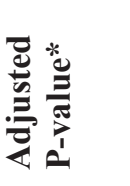 & & $\frac{⿱ 亠}{\stackrel{0}{0}}$ & $\vec{\sigma}$ & సે & 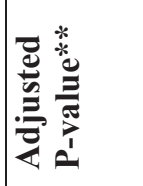 & I \\
\hline 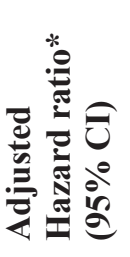 & & 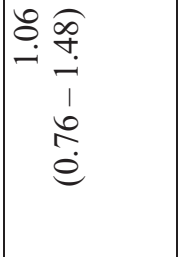 & 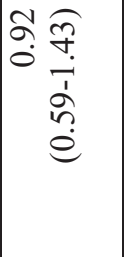 & 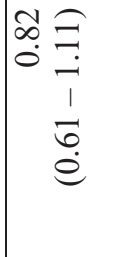 & 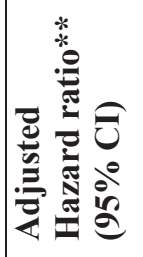 & 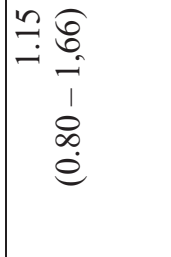 \\
\hline 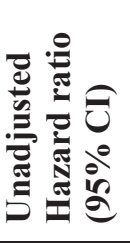 & & 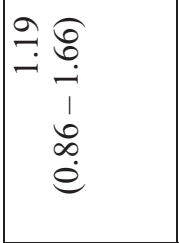 & o: & $\begin{array}{ccc}\infty & 0 \\
\infty & \infty \\
0 & \stackrel{1}{1} \\
1 & 1 \\
0 & 0 \\
0 & 0\end{array}$ & 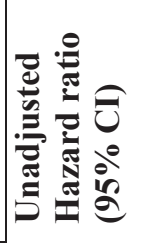 & 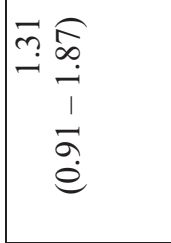 \\
\hline 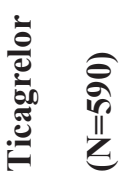 & & 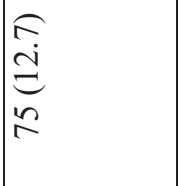 & $\begin{array}{l}\partial \\
\dot{\infty} \\
\dot{f} \\
\dot{f}\end{array}$ & 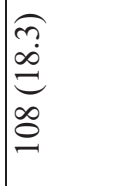 & 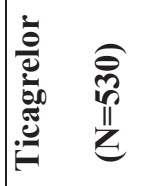 & 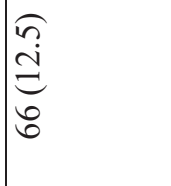 \\
\hline 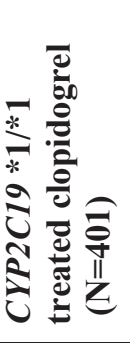 & & 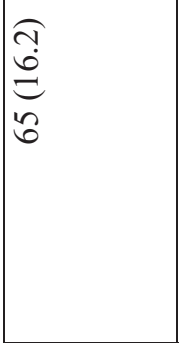 & $\begin{array}{l}\hat{n} \\
\infty \\
\infty \\
\dot{m}\end{array}$ & 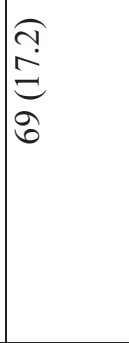 & 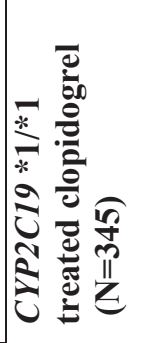 & $\begin{array}{l}\hat{\sigma} \\
\stackrel{n}{2} \\
i n \\
i n\end{array}$ \\
\hline 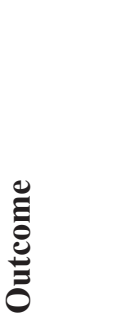 & 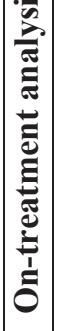 & 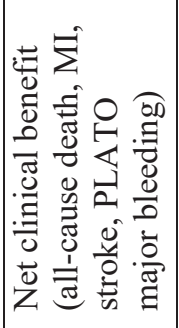 & 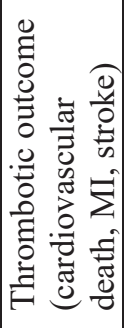 & 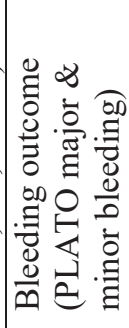 & 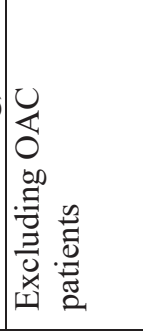 & 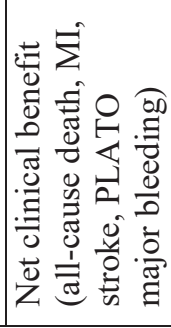 \\
\hline
\end{tabular}



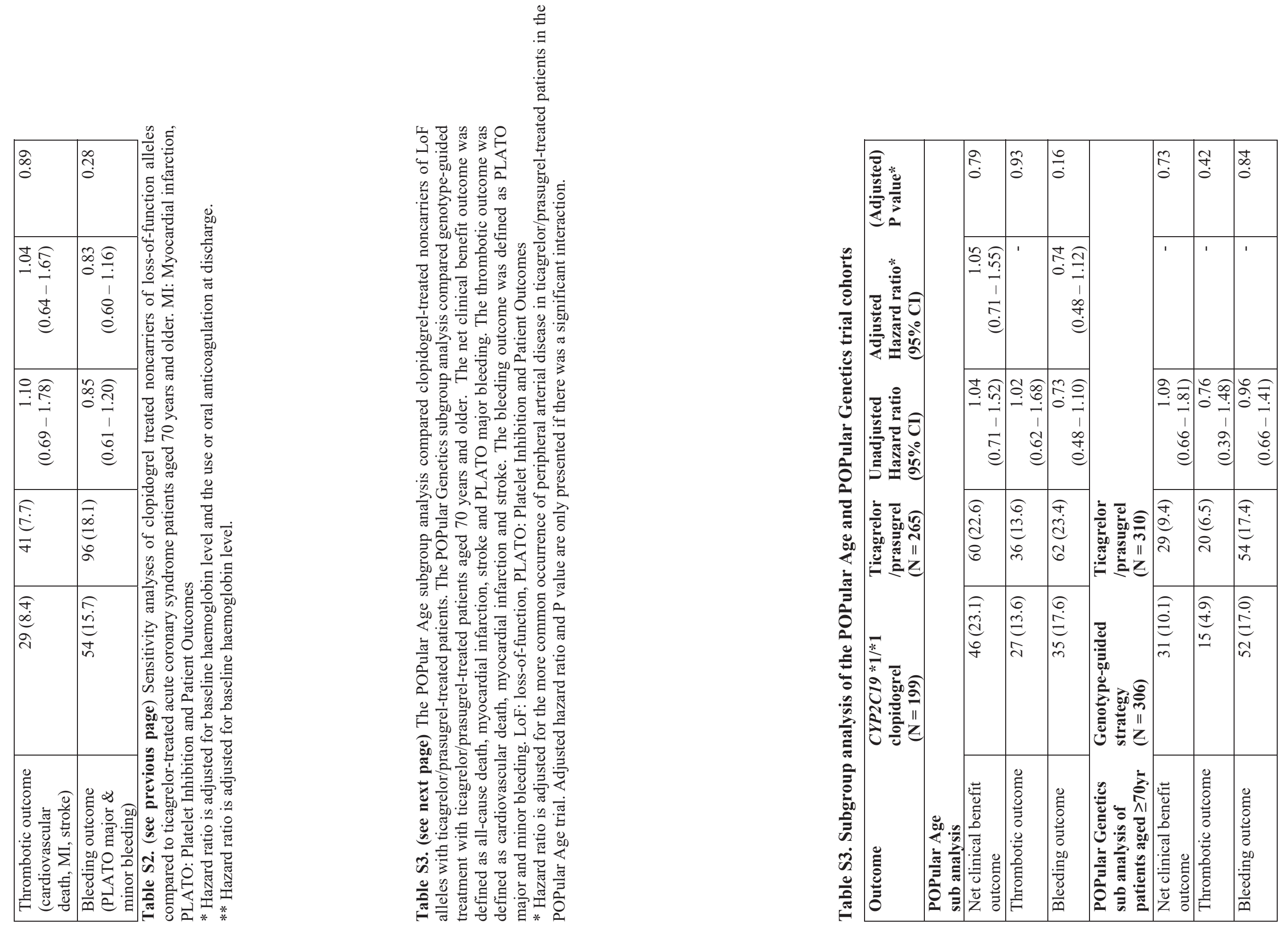
Cost-effectiveness of a CYP2C19 genotypeguided strategy in acute myocardial infarction patients: Results from the POPular Genetics trial

Daniel M.F. Claassens, Pim W.M. van Dorst, Gerrit J.A. Vos, Thomas 0. Bergmeijer, Renicus S. Hermanides, Arnoud W.J. van $t$ Hof, Pim van der Harst, Emanuele Barbato, Carmine Morisco, Richard M. Tjon Joe Gin, Folkert W. Asselbergs, Arend Mosterd, Jean-PaulR. Herrman, Willem J.M. Dewilde, Maarten J. Postma, Vera H.M. Deneer, Jurriën M. ten Berg, Cornelis Boersma

American Journal of Cardiovascular Drugs 2021; DOI: 10.1007/s40256021-00496-4

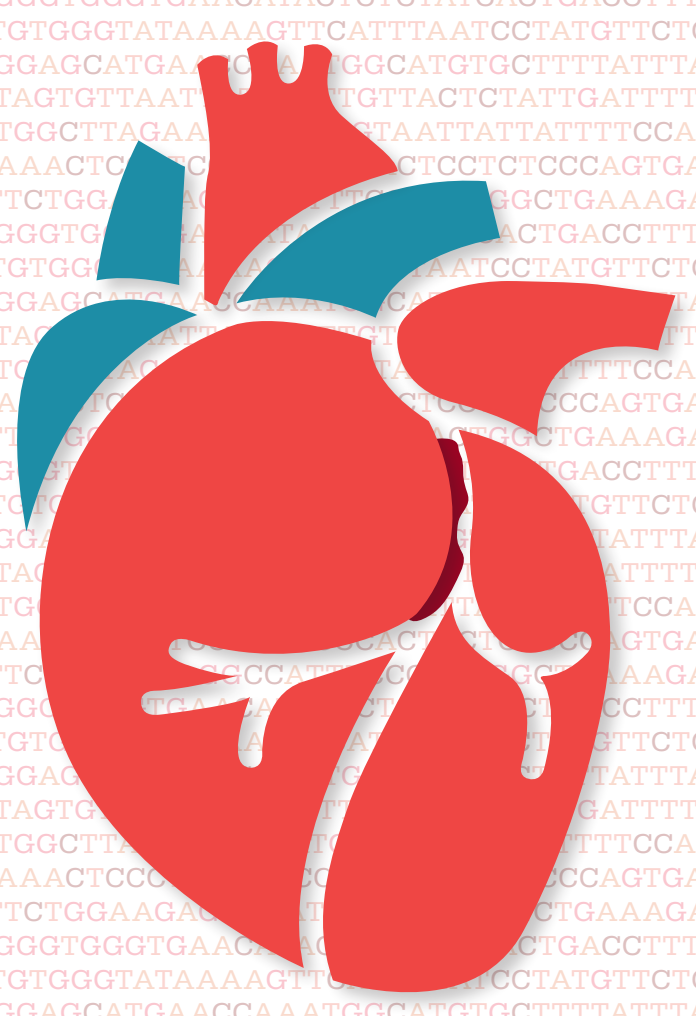




\section{Abstract}

Objective

The POPular Genetics trial demonstrated that a CYP2C19 genotype-guided $\mathrm{P}_{2} \mathrm{Y}_{12}$ inhibitor strategy reduced bleeding rates compared to standard treatment with ticagrelor or prasugrel, without increasing thrombotic event rates after primary percutaneous coronary intervention (PCI). In this analysis, we aimed to evaluate the cost-effectiveness of a genotype-guided strategy compared to standard treatment with ticagrelor or prasugrel.

\section{Methods}

A 1-year decision tree based on the POPular Genetics trial in combination with a lifelong Markov model was developed to compare costs and qualityadjusted life years (QALYs) between a genotype-guided and a standard $\mathrm{P}_{2} \mathrm{Y}_{12}$ inhibitor strategy in myocardial infarction patients undergoing primary PCI. The cost-effectiveness analysis was conducted from a Dutch healthcare system perspective. Within-trial survival and utility data were combined with lifetime projections to evaluate lifetime costs-effectiveness for a cohort of 1000 patients. Costs and utilities were discounted at $4 \%$ and $1.5 \%$ respectively according to Dutch guidelines for health-economic studies. Besides deterministic and probabilistic sensitivity analyses, several scenario analyses (different time horizons, different discount rates, equal prices for $\mathrm{P} 2 \mathrm{Y}_{12}$ inhibitors and equal distribution of thrombotic events between the two strategies) were conducted.

\section{Results}

Base-case analysis with a hypothetical cohort of 1000 subjects, demonstrated 8.98 QALYs gained and $€ 725,550.69$ cost-savings for the genotype-guided strategy (dominant). The deterministic and probabilistic sensitivity analysis confirmed the robustness of the model and the costeffectiveness results. In scenario analyses, the genotype-guided strategy remained dominant.

\section{Conclusion}

In patients undergoing primary PCI, a CYP2C19 genotype-guided strategy compared to standard treatment with ticagrelor or prasugrel resulted in QALYs gained and cost-savings.

\section{Introduction}

Patients with myocardial infarction (MI) and patients undergoing percutaneous coronary intervention $(\mathrm{PCI})$ are treated with dual antiplatelet therapy consisting of aspirin and a $\mathrm{P} 2 \mathrm{Y}_{12}$ inhibitor. Guidelines favour ticagrelor and prasugrel over clopidogrel in MI patients due to a reduction in thrombotic events $[1,2]$. One of the reasons for the reduced effectiveness of clopidogrel compared to the other $\mathrm{P}_{2} \mathrm{Y}_{12}$ inhibitors is that clopidogrel has to be converted into its active metabolite. The most important enzyme in this process is the CYP $4502 \mathrm{C} 19$ enzyme. This enzyme is encoded by the CYP2C19 gene, of which more than 30 different alleles have been identified [3]. Some of these alleles encode for an enzyme that is no functional. The CYP2C19*2 and *3 alleles are the most common loss-offunction alleles and almost $1 / 3$ of the people in western populations carry one or two of these alleles [4]. Several studies demonstrated that these patients have an increased risk of developing major adverse cardiac events [5]. This has prompted the Food and Drug administration (FDA) to add a boxed warning for clopidogrel, describing that the drug might not be effective in patients carrying two loss-of-function alleles [6]. Nevertheless, clopidogrel is still the most frequently prescribed $\mathrm{P} 2 \mathrm{Y}_{12}$ inhibitor, either due to fear of bleeding complications or other side effects when using the stronger $\mathrm{P}_{2} \mathrm{Y}_{12}$ inhibitors or for economic reasons (e.g. lower costs of treatment) [7-9].

The Patient Outcomes after primary PCI (POPular) Genetics trial showed in patients with ST-segment elevation myocardial infarction (STEMI) undergoing primary $\mathrm{PCI}$, that a $C Y P 2 C 19$ genotype-guided $\mathrm{P}_{2} \mathrm{Y}_{12}$ inhibitor strategy was non-inferior for a net clinical benefit outcome while reducing bleeding outcomes as compared to standard treatment with ticagrelor and prasugrel [10]. Since genetic testing is associated with higher costs, while clopidogrel has a lower price than the other $\mathrm{P}_{2} \mathrm{Y}_{12}$ inhibitors, the objective of this study was to assess the cost-effectiveness of the genotype-guided strategy compared to a standard treatment strategy with ticagrelor or prasugrel, within the context of the Dutch healthcare system.

\section{Methods}

Study design

Details on the methods and results of the POPular Genetics trial have been reported previously [10,11]. In brief, POPular Genetics was an open label randomized, multicentre trial with 2,488 STEMI patients undergoing primary PCI. It compared a standard treatment with the $\mathrm{P}_{2} \mathrm{Y}_{12}$ inhibitors ticagrelor (90mg twice daily) or prasugrel (5 or $10 \mathrm{mg}$ once daily based on 
the summary of product characteristics approved by the European Medicines Agency (EMA)) with a CYP2C19 genotype-guided strategy, where patients without $C Y P 2 C 19 * 2$ or $* 3$ loss-of-function alleles were prescribed clopidogrel ( $75 \mathrm{mg}$ once daily), and ticagrelor or prasugre (dosage identical to standard treatment) if they were carrier of such a lossof-function allele. All patients also received guideline recommended aspirin. Treatment and follow-up duration were 12 months. At 1, 6 and 12 months all patients received a questionnaire either by mail or email. This questionnaire contained the EQ-5D-5L, which was used to calculate health utilities. The cost-effectiveness analysis was pre-specified as part of the trial protocol. The trial was approved by the appropriate ethics committees and national authorities. All patients provided informed consent.

\section{Model overview}

The current analysis was designed to calculate the cost-effectiveness of a CYP2C19 genotype-guided strategy for STEMI patients undergoing primary PCI. A two-part decision-analytic model was developed, comprising of a 1-year decision tree to determine the initial distribution of the cohort over the Markov states (Figure 1A) and a Markov model to simulate life-long costs and effects (Figure 1B). In the 1-year decision tree, all patients could experience a minor or major bleeding independent of experiencing any of the other events. At the end of the 1-year decision tree period, patients would enter the respective Markov states depending on the experienced event (e.g. recurrent MI, recurrent stroke or death). The Markov-model structure is identical to previously published trials investigating antiplatelet therapy in similar populations [12], clinically validated and adjusted to allow for recurrence of stroke and MI. Four disease transient states were included reflecting the lifetime progression of individuals after STEMI, including MI, post-MI, stroke and post-stroke. Additionally, the model comprised two absorbing states, defined as cardiovascular (CV) death and non-CV death. A cohort of 1000 hypothetical subjects was used, to simulate the progression through the different disease states. Subjects could switch between disease states or remain in the same disease state, based on transition probabilities. In the base case analysis, a lifetime horizon was used with a cut-off at the age of 100

\section{Model assumptions}

One of the assumptions underpinning the model was that patients in both groups were treated with aspirin monotherapy after the 1-year trial period was finished. Therefore, no difference in bleeding rates was expected in the Markov model. Furthermore, bleeding usually decreases quality of

\section{Figure 1. Cost-effectiveness model}

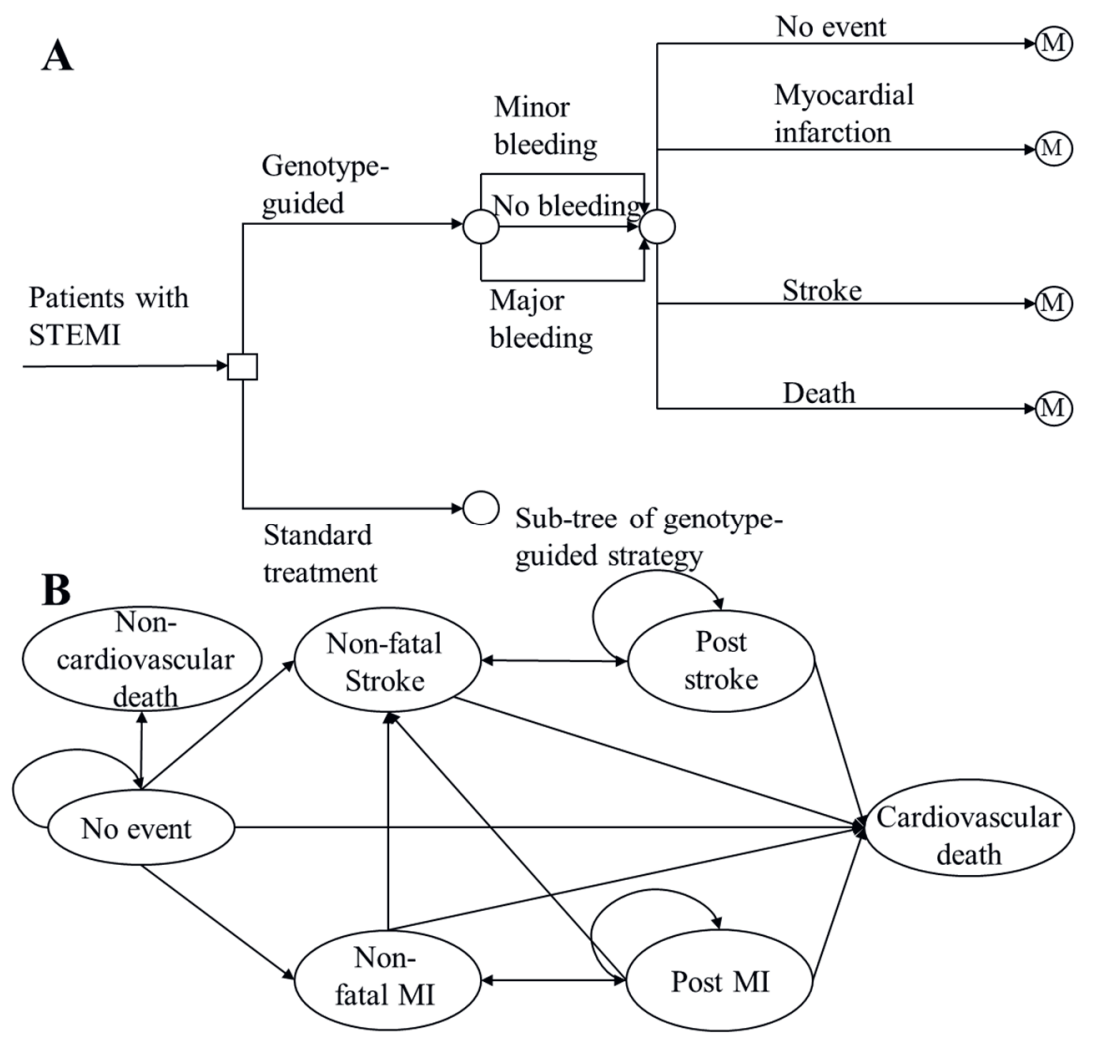

Figure 1. Model structure. Figure 1A: 1-year decision tree based on the POPular Genetics trial. Figure 1B: Long-term Markov model. MI: myocardial infarction.

life for only a short period of time. Hence, bleeding was not included as a separate health state in the Markov model. It was assumed that patients could not develop multiple events during the 1-year trial, which was in line with the Platelet Inhibition and Patient Outcomes (PLATO) trial costeffectiveness analysis [12]. In addition, the assumption was made that 
recurrent stroke or MI could only happen with a minimum interval of 1 year.

\section{Population}

The intention-to-treat population from the POPular Genetics trial was used for the current decision-analytic model (Table 1). The mean age of the tria population was 61 years old, $15 \%$ was 75 years and older, $25 \%$ was female and $10 \%$ had a prior history of coronary artery disease. In line with the mean age of the patients in the POPular Genetics trial cohort, all individuals were at the age of 61 at the start of the decision tree.

\section{Model input parameters}

\section{Transition probabilities}

All model inputs are presented in Table 2. Probabilities for all patients in the 1-year decision tree were based on the results from the POPular Genetics trial [10]. At the end of the decision tree, patients were allocated to their respective health state in the long-term Markov model. Starting from the second year, a Markov state-transition model with yearly cycles was used to simulate disease progression of patients over their lifetime. In each health state, patients could experience an MI, stroke or death in any year. Patients in the 'Post-MI' and 'Post-stroke' health states had a higher risk of subsequent events than patients in the 'No-event' health state. The transition probabilities of experiencing subsequent events were derived by multiplying the baseline probabilities by the relative risk factors (Table 2) [12]. For mortality, an age specific mortality rate was used based on the Dutch population lifetables. It was not possible to transition from 'poststroke' to 'post-MI', since the MI health state had a lower risk and was less costly than the stroke health state.

\section{Costs}

Cost-effectiveness was estimated from the healthcare perspective, so only medical costs were included. Costs were inflated to 2020 using the consumer price index inflation from the Dutch Central Bureau of Statistics (Table 2). Costs were based on the Dutch healthcare system and obtained from literature or Dutch governmental agencies. Cost categories were treatment costs (genetic test and different antiplatelet drugs) and costs associated with the different events: minor bleeding, major bleeding, nonfatal MI, nonfatal stroke, post-MI, post-stroke and death. The cost associated with the use of aspirin and other medication was excluded from the analysis, since it was assumed to affect both treatments strategies equally. Costs were discounted using an annual rate of $4 \%$ in accordance with existing guidelines for conducting health-economic evaluations [23].

\section{Health utilities}

Health utilities, measured in quality-adjusted life years (QALYs), were dependent on the events experienced by patients (Table 2). Health utility estimates were either derived from the POPular Genetics trial (using the EQ-5D-5L questionnaire) or, in case data was not available from the trial, utility estimates were derived from literature focused on the Dutch healthcare system with similar populations. Based on previously published literature, bleeding let to a temporary disutility for the duration of the event during the first year following treatment. QALY's were discounted using an annual rate of $1.5 \%$ in accordance with Dutch guidelines for conducting health-economic evaluations [23]

\section{Outcomes}

The outcome measures to compare the two strategies in this study were costs, QALYs and the incremental cost-effectiveness ratios (ICERs) presented as cost per QALY gained.

\section{Sensitivity and scenario analysis}

The base-case analysis was based on the model inputs as shown in Table 2. To accommodate for the model uncertainty, univariate deterministic sensitivity analyses and probabilistic sensitivity analyses were conducted For the sensitivity analysis, the estimated range of each parameter was based on the $95 \% \mathrm{CI}$ in the studies. If $95 \% \mathrm{CI}$ was not available, ranges were calculated with a standard error of the mean of $25 \%$. For univariate deterministic sensitivity analyses, each of the parameters was varied one by one over the $95 \% \mathrm{CI}$, to examine the influence of individual parameters on the ICER. In the probabilistic sensitivity analysis, a Monte Carlo simulation was performed (1,000 iterations) by varying the parameters simultaneously over their $95 \%$ CI. The cost, probability and utility parameter distributions were varied with a Gamma-, Pert and Beta distribution, respectively. Outcomes of the sensitivity analysis are presented in a tornado-diagram and a cost-effectiveness plane. Four additional scenarios were conducted to capture the effect of the time horizon $(1,5,10$ and 25 years in the Markov model (scenario 1), adjustment of the discount rates to both costs and utilities at $4 \%$ (scenario 2 ), equal drug prices for all three drugs at $€ 0.05 /$ day, to mimic the availability of generic variants in the future (scenario 3) and equa distribution of the cohort over 'post-MI', 'post-stroke' and 'death' (both $\mathrm{CV}$ death and non-CV death) at the start of the Markov-model for both strategies to account for the uncertainty that a genotype-guided strategy does not result in numerically less stroke and MI as seen in the POPular Genetics trial (scenario 4). 
Table 1. POPular Genetics trial population baseline characteristics

\begin{tabular}{|c|c|c|}
\hline Characteristics & $\begin{array}{r}\text { Genotype-guided } \\
(\mathrm{N}=1242)\end{array}$ & $\begin{array}{r}\text { Standard } \\
\text { treatment } \\
(\mathrm{N}=1246)\end{array}$ \\
\hline Mean age - yr & $61.9 \pm 11.1$ & $61.4 \pm 11.5$ \\
\hline Age $\geq 75$ years - no. $(\%)$ & $188(15.1)$ & $175(14.0)$ \\
\hline Female sex - no. (\%) & $317(25.5)$ & $309(24.8)$ \\
\hline Mean body-mass index ${ }^{\mathrm{a}}$ & $27.5 \pm 6.67$ & $27.0 \pm 4.27$ \\
\hline $\begin{array}{l}\text { Creatinine clearance } \\
<60 \mathrm{ml} / \text { minute at baseline }^{\mathrm{b}}-\text { no. } \\
(\%)\end{array}$ & $121(9.8)$ & $109(8.8)$ \\
\hline \multicolumn{3}{|l|}{ Medical history -- no. (\%) } \\
\hline Current smoker & $562(45.8)$ & $565(45.8)$ \\
\hline Diabetes Mellitus & $150(12.1)$ & $138(11.1)$ \\
\hline Arterial hypertension & $521(42.0)$ & $511(41.0)$ \\
\hline Hyperlipidaemia & $260(20.9)$ & $255(20.5)$ \\
\hline $\begin{array}{l}\text { History of coronary artery } \\
\text { disease }\end{array}$ & $133(10.7)$ & $118(9.5)$ \\
\hline Peripheral arterial disease & $39(3.1)$ & $34(2.7)$ \\
\hline History of bleeding & $30(2.4)$ & $23(1.9)$ \\
\hline \multicolumn{3}{|c|}{ Angiographic and procedural characteristics } \\
\hline \multicolumn{3}{|c|}{\begin{tabular}{l|l} 
Number of diseased coronary & \\
vessels $\geq 50 \%-$ no. $(\%)$ &
\end{tabular}} \\
\hline 1 & $634(51.2)$ & $675(54.2)$ \\
\hline 2 & $417(33.7)$ & $376(30.2)$ \\
\hline 3 & $188(15.1)$ & $194(15.6)$ \\
\hline Ostial lesion - no. (\%) & $76(6.4)$ & $65(5.5)$ \\
\hline Bifurcation lesion - no. (\%) & $214(18.1)$ & $239(20.2)$ \\
\hline \multicolumn{3}{|l|}{ Stent type - no. $(\%)$} \\
\hline Bare metal stent & $60(4.8)$ & $50(4.0)$ \\
\hline $\begin{array}{l}\text { Bioresorbable vascular } \\
\text { scaffold }\end{array}$ & $9(0.7)$ & $16(1.3)$ \\
\hline Drug eluting stent & $1167(94.0)$ & $1174(94.2)$ \\
\hline Plain old balloon angioplasty & $17(1.4)$ & $23(1.9)$ \\
\hline Total stent length - mm & $27.0 \pm 14.8$ & $28.0 \pm 15.3$ \\
\hline \multicolumn{3}{|l|}{ Medication at discharge - no. (\%) } \\
\hline Aspirin & $1211(97.7)$ & $1208(97.4)$ \\
\hline ADP receptor antagonist & $1237(99.8)$ & $1237(99.8)$ \\
\hline Clopidogrel & $688(55.5)$ & $91(7.3)$ \\
\hline Prasugrel & $15(1.2)$ & $27(2.2)$ \\
\hline Ticagrelor & $534(43.1)$ & $1119(90.2)$ \\
\hline (Novel) oral anticoagulation & $14(1.1)$ & $9(0.7)$ \\
\hline
\end{tabular}

Table 1. Baseline characteristics of the POPular Genetics trial population a: BMI is the weight in kilograms divided by the square of the height in meters. b: The creatinine clearance was calculated using the CKD-EPI formula.

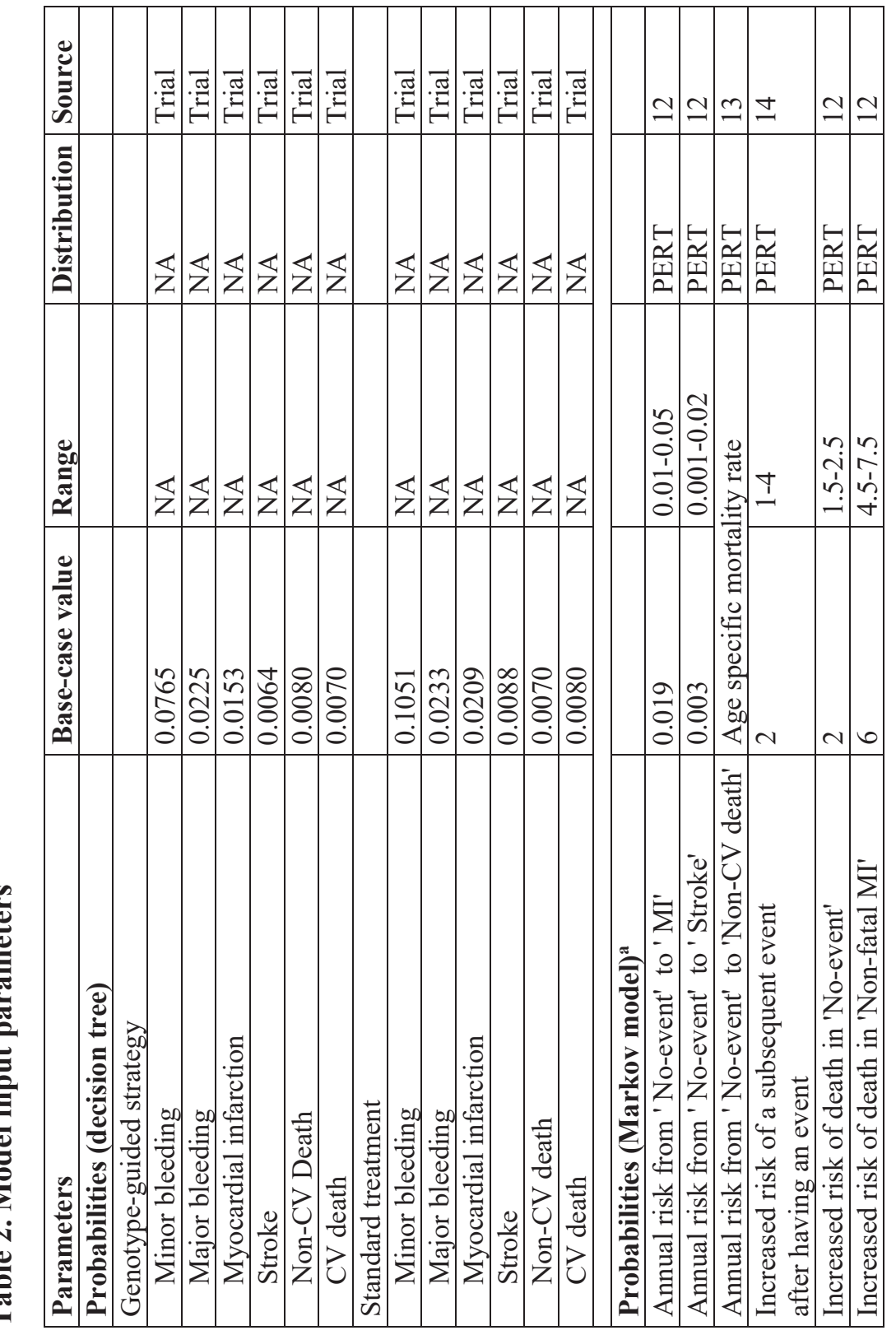



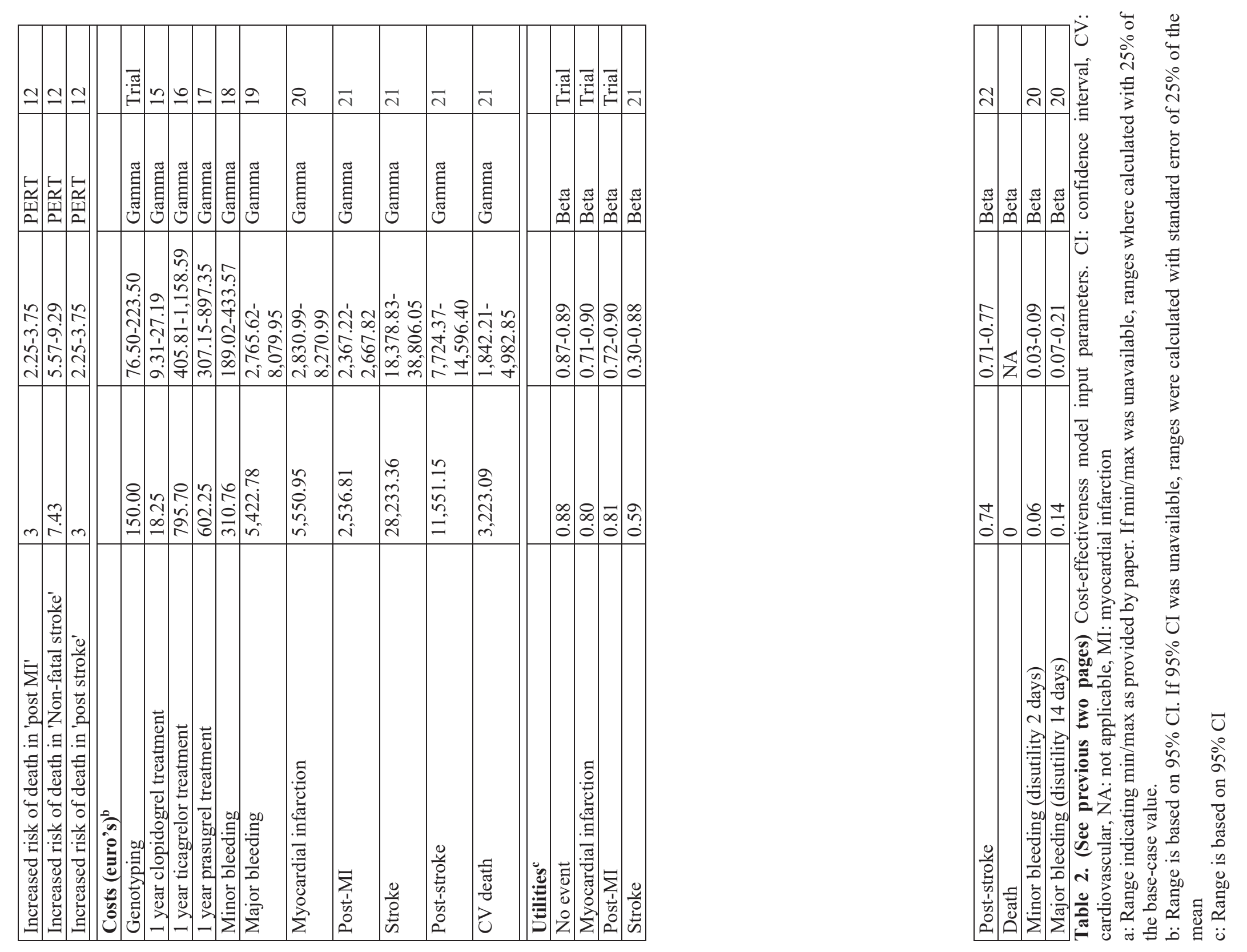


\section{Results}

\section{Base-case analysis}

For a cohort of 1000 patients undergoing primary PCI based on the POPular Genetics trial, the genotype-guided strategy resulted in a gain in QALYs of 8.98 while saving $€ 725,550.69$ (0.009 QALYs gained and $€ 725.56$ saved per patient) (Table 3). Cost-saving results for the genotypeguided strategy indicate that this strategy dominates current standard treatment without genotyping. In Figure 2 , results of the univariate deterministic sensitivity analysis are displayed in a tornado diagram. The results demonstrate that when varying the different model inputs over their confidence intervals, the genotype-guided strategy remains cost-saving. Results of the 1000 iterations of the probabilistic sensitivity analysis were plotted on a cost-effectiveness plane (Figure 3). The genotype-guided strategy was cost-saving in each iteration of the Monte Carlo simulation, while it increased QALYs in almost all iterations.

\section{Scenario analyses}

In Table 3 results are presented for the varying time horizons (scenario 1), adjustment in discount rates (scenario 2), equal prices for all $\mathrm{P} 2 \mathrm{Y}_{12}$ inhibitors (scenario 3) and an equal distribution of patients amongst health states between the two strategies (scenario 4). In all different scenarios, the genotype-guided strategy remained cost saving and improved QALY's.

\section{Discussion}

In this prospectively designed cost-effectiveness analysis, based on the POPular Genetics trial data, a CYP2C19 genotype-guided strategy was associated with an increase in QALYs and was cost-saving (dominant) as compared to standard treatment with ticagrelor or prasugrel in STEMI patients undergoing primary PCI. The robustness of this finding was confirmed by various additional sensitivity and scenario analyses. In the Netherlands, the willingness to pay threshold varies between $€ 20.000$ to $€ 80.000$ depending on the intervention, while the willingness to pay threshold in other European countries varies as well, but is generally of the same magnitude [24]. Our results are well below the lower end of this range. In the United States, a guideline on health economic analyses was published by the American College of Cardiology (ACC)/American Heart Association (AHA) [25]. According to this guideline, our findings suggest a high value of a genotype-guided strategy as compared to a standard treatment strategy with ticagrelor or prasugrel, since it improved QALYs while reducing costs.
The cost-effective results showing that the genotype-based strategy is dominant, were primarily driven by two differences in the first year. The first determining variable is the price for generic clopidogrel which is lower (€0.05/day) compared to the patented ticagrelor and prasugrel ( $€ 2.18 /$ day and $€ 1.65 /$ day, respectively) [15-17]. The difference in costs between clopidogrel and the other $\mathrm{P}_{2} \mathrm{Y}_{12}$ inhibitors reflects the potential additional cost of performing a genetic test that was estimated to be $€ 150.00$ for a point-of-care test. Since both ticagrelor and prasugrel are expected to have generic variants available in the next few years, which will likely cause a drop in prices, a scenario analysis was conducted in which the daily costs of ticagrelor and prasugrel were identical to clopidogrel costs $(€ 0.05 /$ day). Following this scenario analysis, the genotype-guided strategy remained cost-saving, with similar QALYs gained as compared to the base-case analysis. Therefore, it can be realistically assumed that extra costs associated with performing genetic testing will not change the results. The availability of generic ticagrelor and prasugrel will lower prices in the future, but it is uncertain to what extent. The scenario analysis performed is therefore on the conservative end of the spectrum. The second important factor driving the results of the current analysis is the distribution of the patients at the onset of the Markov model. Based on the results from the POPular Genetics trial, patients in the genotype-guided arm were in a more favourable health-state than patients in the standard treatment arm when they entered the Markov model, due to a lower incidence of stroke and $\mathrm{MI}$ in the genotype-guided cohort as compared to the standard treatment cohort. To account for the uncertainty that a genotype-guided strategy actually leads to numerically less stroke and less MI, as seen in the POPular Genetics trial, a scenario analysis was conducted in which there was no difference amongst the distribution of patients in the different health states when entering the Markov model between the two strategies. In this scenario analysis, the genotype-guided strategy remained cost saving while only a small difference in QALYs gained remained. This was expected, since the difference in QALYs is now only caused by a difference in minor bleeding events between both groups, which have a relatively low impact on long-term health utilities. These results demonstrate that the cost-savings and long-term benefits are based on the differences in the first year after genotype-guided treatment.

In the POPular Genetics trial, more than $95 \%$ of the included patients was Caucasian, while only a small proportion of patients was of Asian, Latin American or African descent [10]. The prevalence of CYP2C19 loss-offunction alleles does not differ much between Africans, Americans and 

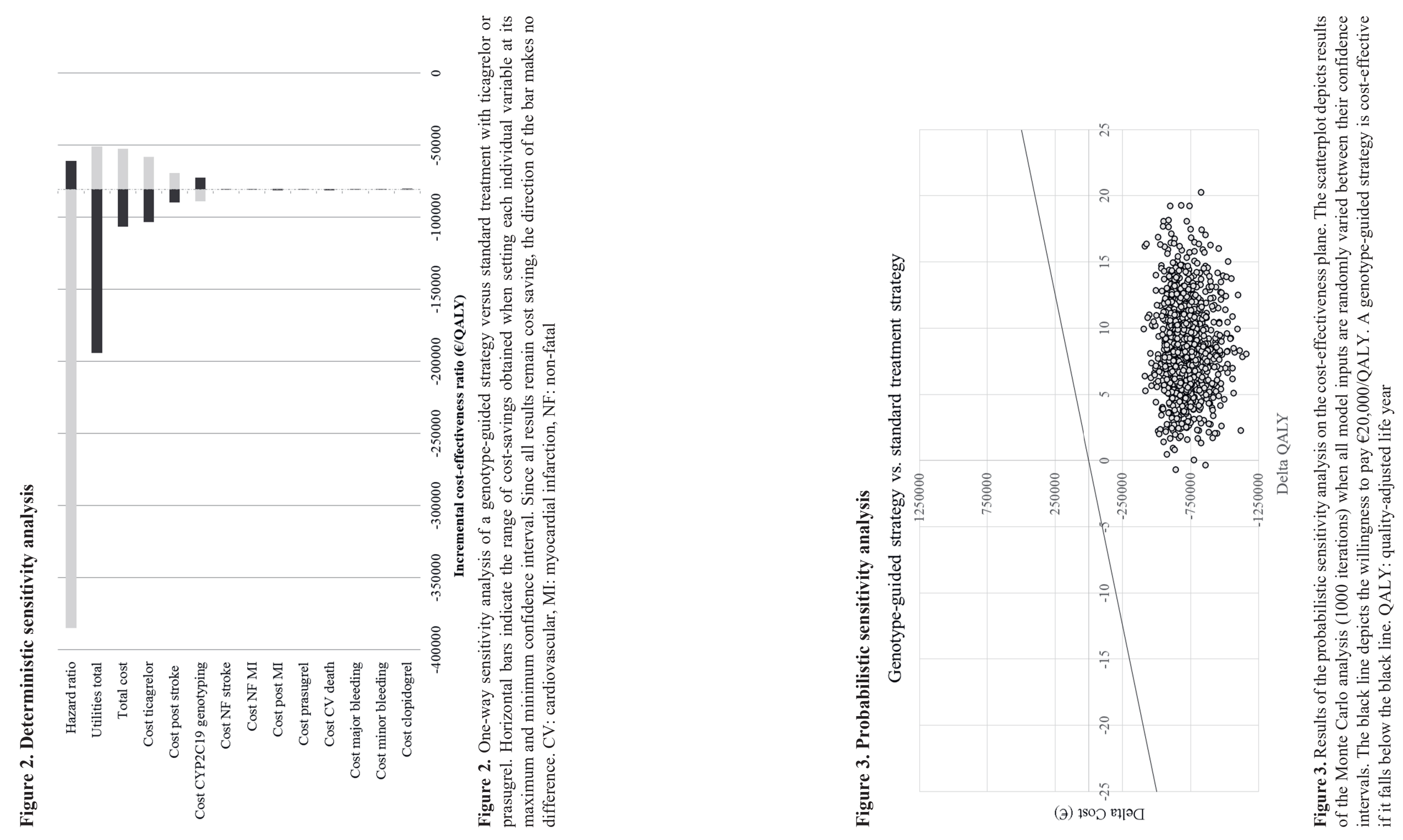


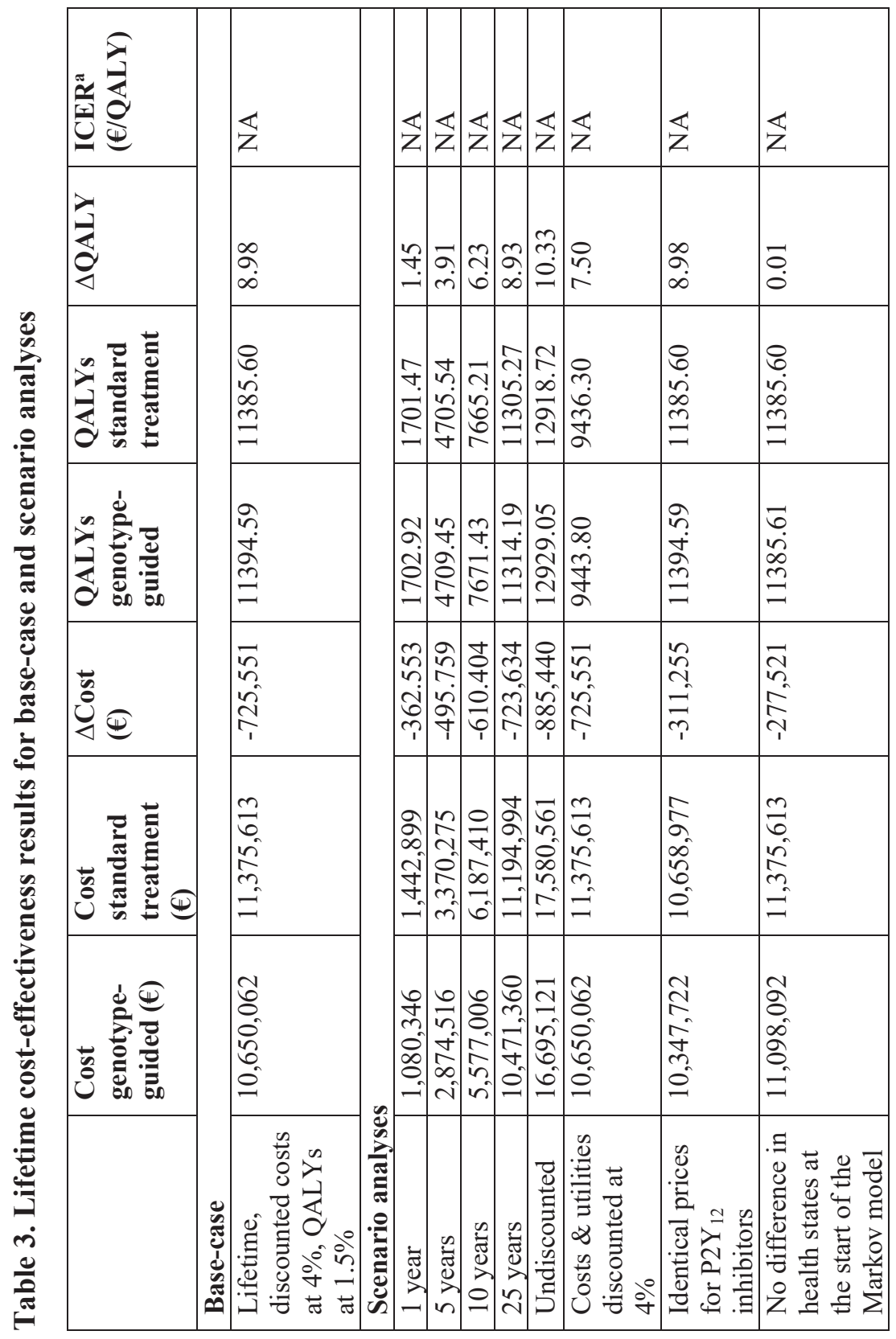

Table 3. (see previous page) Cost-effectiveness analysis outcomes of the basecase and sensitivity analyses based on the POPular Genetics trial.

a: When a genotype-guided strategy resulted in cost-savings, the ICER could not be calculated.

ICER: incremental cost-effectiveness ratio, NA: not applicable, QALY: qualityadjusted life years

Europeans, but is a lot higher in Asian populations [4]. A higher

prevalence of loss-of-function alleles means more people will remain on ticagrelor or prasugrel, which will negatively affect the results of the cost-effectiveness analysis in these countries. In addition, it is uncertain how this affects outcomes of the trial. On the other hand, in countries with a similar prevalence of loss-of-function alleles, one would expect similar clinical outcomes as in our trial and similar results of a cost-

effectiveness analysis. However, we should be aware that healthcare costs can differ significantly between countries.

\section{Comparison with other studies}

Several health economic analyses concerning a genotype-guided $\mathrm{P} 2 \mathrm{Y}_{12}$ inhibitor strategy have been published in the past [14,26-29]. These analyses used extrapolated data from for instance the PLATO or Trial to

Assess Improvement in Therapeutic Outcomes by Optimizing Platelet Inhibition with Prasugrel-Thrombolysis in Myocardial Infarction (TRITON TIMI) 38 trial to build their decision tree, but they did not have data from a trial specifically investigating a genotype-guided strategy. A study by Lala et al., who based most of their model input parameters on the TRITON TIMI 38, found similar results as presented within the current study [14]. They found a genotype-guided strategy to be cost-saving, and an increase in QALYs compared to both a standard treatment with prasugrel and a standard treatment with clopidogrel. Our results are also in accordance with the cost-effectiveness analysis by Wang et al., which used data from the PLATO trial and found a genotype-guided strategy to be cost-saving and with improving QALYs as compared to standard treatment with ticagrelor [26]. In addition, they also concluded that compared to a standard treatment with clopidogrel, a genotype-guided strategy improved 
QALYs at an ICER of \$2560. A cost-effectiveness analysis by Limdi et al. investigated whether universal ticagrelor treatment or genotype-guided deescalation was cost-effective in acute coronary syndrome patients undergoing PCI as compared to universal clopidogrel treatment based on real-world data [27]. While both strategies increased QALYs compared to universal clopidogrel treatment, only genotype-guided de-escalation was cost-effective at an ICER of $\$ 42,365$. Similar as to our results, results from both Wang et al. and Limdi et al. are below the 'high value' limit of $\$ 50.000 / \mathrm{QALY}$ as set by the ACC/AHA guideline [25]

In contrast to our results, two studies by Sorich et al. and Crespin et al., both based on results from the PLATO trial, found that ticagrelor treatment was cost-effective as compared to a genotype-guided strategy [28,29]. This means higher costs with additional QALYs gained. The fact that ticagrelor compared to the genotype-guided strategy resulted in QALYs gained, already explains the major difference between the results of Crespin et al. and Sorich et al. compared to the results of this study [28,29]. In addition, some important considerations with respect to the findings of Crespin et al. and Sorich et al. should be taken into account. Crespin et al. noted that ticagrelor would no longer be cost-effective if the hazard ratio for mortality between ticagrelor and clopidogrel is higher than 0.93 [29], which was the case in the POPular Genetics trial (HR 1.00) [10]. In the study by Sorich et al., the cost of ticagrelor was only 3 times the cost of clopidogrel while the Dutch tariffs indicate a 43.6 times higher cost of ticagrelor compared to clopidogrel [28]. Sorich et al. reported that this small price difference between clopidogrel and ticagrelor was one of the reasons as to why ticagrelor treatment was cost-effective compared to a genotype-guided strategy in their study (ICER of ticagrelor versus a genotype-guided strategy: AUS\$ 22,821). Furthermore, researchers indicated that when the hazard ratio between ticagrelor and clopidogrel would exceed 0.95 , as it does in our study, the ICER would increase to over AUS\$ 50,000, making the cost-effectiveness of ticagrelor compared to a genotype-guided strategy highly questionable.

While the previously mentioned studies used data from the PLATO and TRITON TIMI 38 trial to build their study model, the respective study groups themselves also wrote health economic analyses. The health economic analysis of the PLATO trial, which compared ticagrelor to clopidogrel, noted, like Crespin et al., that most of the QALYs gained in the ticagrelor arm of their analysis were derived from the mortality benefit from ticagrelor as compared to clopidogrel [12]. Most benefit in the TRITON TIMI 38 health economic analysis was derived from a reduction in $\mathrm{MI}$ in the prasugrel arm as compared to the clopidogrel arm [30]. In the POPular Genetics trial, no numerical benefit in both MI and mortality were seen in the standard treatment arm as compared to the genotype-guided arm [10]. Besides the much lower costs of clopidogrel, treatment compared to the other P2Y12 inhibitors and some savings on bleeding events, this is one of the most important reasons as to why there was no benefit for ticagrelor and prasugrel in our analysis.

\section{Limitations}

Our results should be interpreted in light of the following limitations. This cost-effectiveness analysis was based on data from STEMI patients undergoing primary PCI. Therefore, it is unknown if these results also apply to patients with another form of acute coronary syndrome and patients not undergoing PCI. In addition, a majority of the patients in the POPular Genetics trial were treated with ticagrelor. Since the recently published Intracoronary Stenting and Antithrombotic Regimen: Rapid Early Action for Coronary Treatment (ISAR-REACT) 5 trial found that the use of prasugrel might be beneficial over the use of ticagrelor [31], both costs and event rates might be affected if more patients are treated with prasugrel instead of ticagrelor. This applies to both the standard treatment and genotype-guided groups, since $1 / 3$ of patients in the genotyping group are still treated with ticagrelor or prasugrel. Concerning the healtheconomic analysis, the analysis is based on a healthcare perspective instead of a societal perspective, which is sometimes preferred. However, using a societal perspective would mean further assumptions regarding costs that had to be made. Besides, the only difference in our model was present in the first year during the decision tree, hence adding the same costs to both arms (treatment and control) was expected not to add additional value to the model nor affect the result of the analysis.

\section{Conclusion}

In STEMI patients undergoing primary PCI, a CYP2C19 genotype-guided strategy compared to standard treatment with ticagrelor or prasugrel resulted in QALYs gained and cost-savings. 


\section{References}

1. Levine GN, Bates ER, Bittl JA et al. 2016 ACC/AHA guideline focused update on duration of dual antiplatelet therapy in patients with coronary artery disease: a report of the American College of Cardiology/American Heart Association Task Force on Clinical Practice Guidelines. J Am Coll Cardiol 2016; 68:1082-1115

2. Ibanez B, James S, Agewall S, et al. 2017 ESC guidelines for the management of acute myocardial infarction in patients presenting with ST-segment elevation: the task force for the management of acute myocardial infarction in patients presenting with ST-segment elevation of the European Society of Cardiology (ESC). Eur Heart J 2018; 39:119177

3. Scott SA, Sangkuhl K, Shuldiner AR, et al. PharmGKB summary: very important pharmacogene information for cytochrome P450, family 2 , subfamily C, polypeptide 19. Pharmacogenetics and genomics 2012; 22:159-165

4. Scott SA, Sangkuhl K, Stein CM, et al. Clinical Pharmacogenetics Implementation Consortium guidelines for CYP2C19 genotype and clopidogrel therapy: 2013 update. Clinical pharmacology and therapeutics 2013; 94:317-323

5. Mega JL, Simon T, Collet JP, et al. Reduced-function CYP2C19 genotype and risk of adverse clinical outcomes among patients treated with clopidogrel predominantly for PCI: a meta-analysis. JAMA 2010; 304:1821-1830

6. Holmes DR, Dehmer GJ, Kaul S, et al. ACCF/AHA clopidogrel clinical alert: approaches to the FDA "boxed warning": a report of the American College of Cardiology foundation task force on clinical export consensus documents and the American Heart Association endorsed by the Society of Cardiovascular Angiography and Interventions and the Society of Thoracic Surgeons. J Am Coll Cardiol 2010; 56:321-341

7. Motovska Z, Hlinomaz O, Kala P, et al. 1-year outcomes of patients undergoing primary angioplasty for myocardial infarction treated with prasugrel versus ticagrelor. JACC 2018; 71:371-381

8. Gimbel ME, Qaderdan K, Willemsen L, et al. Clopidogrel versus ticagrelor or prasugrel in patients aged 70 years or older with non-STelevation acute coronary syndrome (POPular AGE): the randomised, open-label, non-inferiority trial. Lancet 2020; 395:1374-1381

9. Zettler ME, Peterson ED, McCoy LA, et al. Switching of adenosine diphosphate receptor inhibitor after hospital discharge among myocardia infarction patients: insights from the treatment with adenosine diphosphate receptor inhibitors: Longitudinal assessment of treatment patterns and events after acute coronary syndrome (TRANSLATE-ACS) observational study. Am Heart J 2017; 183:62-68

10. Claassens DMF, Vos GJA, Bergmeijer TO, et al. A genotype-guided strategy for oral P2Y12 inhibitors in primary PCI. N Engl J Med 2019, 381:1621-1631

11. Bergmeijer TO, Janssen PWA, Schipper JC, et al. CYP2C19 genotypeguided antiplatelet therapy in ST-segment elevation myocardial infarction patients-Rationale and design of the patient outcome after primary PCI (POPular) Genetics study. Am Heart J 2014; 168:16-22.e1

12. Nikolic E, Janzon M, Hauch O, et al. Cost-effectiveness of treating acute coronary syndrome patients with ticagrelor for 12 months: results from the PLATO study. Eur Heart J 2012; 34:220-228

13. Central Bureau for Statistics. Lifetables. https://opendata.cbs.nl/statline/\#/CBS/nl/dataset/83482NED/table?ts=16 03871419734 Accessed 15 Oct 2020

14. Lala A, Berger JS, Sharma G, et al. Genetic testing in patients with acute coronary syndrome undergoing percutaneous coronary intervention: a cost-effectiveness analysis. J Thromb Haemost 2013; 11:81-91

15. Zorginstituut Nederland. GIPdatabank: Vergoeding per DDD 2015-2019 voor ATC-subgroep B01AC04 : Clopidogrel. https://www.gipdatabank.nl/databank?infotype $=$ g\&label $=00$ totaal\&tabel_g_00-totaal=B 01-

basis $\& g e g=v g \&$ spec $=$ vg ddd \&item $=$ B01AC04 Accessed 15 Oct 2020

16. Zorginstituut Nederland. GIPdatabank: Vergoeding per DDD 2015-2019 voor ATC-subgroep B01AC24 : Ticagrelor https://www.gipdatabank.nl/databank?infotype $=$ g\&label $=00$ totaal\&tabel g 00 -totaal $=$ B 01 -

basis\&geg $=$ vg\&spec $=$ vg_ddd $\&$ item $=$ B01AC24 Accessed 15 Oct 2020

17. Zorginstituut Nederland. GIPdatabank: Vergoeding per DDD 2015-2019 voor ATC-subgroep B01AC22: Prasugrel. https://www.gipdatabank.nl/databank?infotype $=$ g\&label $=00$ totaal\&tabel_g_00-totaal=B_01-

basis\&geg=vg\&spec $=$ vg_ddd\&item $=$ B01 AC22 Accessed 15 Oct 2020

18. Jacobs MS, De Jong LA, Postma MJ, et al. Health economic evaluation of rivaroxaban in elective cardioversion of atrial fibrillation. Eur J Health Econ 2018; 19:957-965

19. ten Cate-Hoek AJ, Toll DB, Buller HR, et al. Cost-effectiveness of ruling out deep venous thrombosis in primary care versus care as usual. J Thromb Haemost 2009; 7:2042-2049

20. Stevanovic J, Pompen M, Le HH, et al. Economic Evaluation of Apixaban for the Prevention of Stroke in Non-Valvular Atrial Fibrillation in the Netherlands. PLoS One 2014; 9:e103974 
21. De Jong LA, Groeneveld J, Stefanovic J, et al. Cost-effectiveness of apixaban compared to other anticoagulants in patients with atrial fibrillation in the real-world and trial settings. PloS one 2019; 14.9:e 0222658

22. Van Eeden M, Van Heugten C, Van Mastrigt GAPG et al. The burden of stroke in the Netherlands: estimating quality of life and costs for 1 year poststroke. BMJ open 2015; 5:e008220

23. Ijzerman MJ, Al MJ, De Boer A, et al. Richtlijn voor het uitvoeren van economische evaluaties in de gezondheidszorg. Zorginstituut Ned. 2016;1-38

https://www.zorginstituutnederland.nl/publicaties/publicatie/2016/02/29 /richtlijn-voor-het-uitvoeren-van-economische-evaluaties-in-de-

gezondheidszorg. Accessed 15 Oct 2020

24. Ligtenberg G, Staal PC, Goettsch WG, et al. Kosteneffectiviteit in de zorg, op weg naar een genuanceerd en geaccepteerd gebruik van kosteneffectiviteitsgegevens in de zorg. College voor Zorgverzekeringen 2013;1-41

https://www.zorginstituutnederland.nl/binaries/zinl/documenten/rapport /2013/09/30/kosteneffectiviteit-in-de-

zorg/Kosteneffectiviteit+in+de+zorg.pdf Accessed 15 Oct 2020

25. Anderson JL, Heidenreich PA, Barnett PG, et al. ACC/AHA statement on cost/value methodology in clinical practice guidelines and performance measures: a report of the American College of Cardiology/American Heart Association Task Force on Performance Measures and Task Force on Practice Guidelines. J Am Coll Cardio 2014; 63:2304-2322

26. Wang Y, Yan BP, Liew D, LEE VWY. Cost-effectiveness of cytochrome $\mathrm{P} 4502 \mathrm{C} 19 * 2$ genotype-guided selection of clopidogrel or ticagrelor in Chinese patients with acute coronary syndrome. Pharmacogenomics J 2018; 18:113-120

27. Limdi NA, Cavallari LH, Lee CR, et al. Cost-effectiveness of CYP2C19 guided antiplatelet therapy in patients with acute coronary syndrome and percutaneous coronary intervention informed by real-world data. Pharmacogenomics J 2020; 20:724-735

28. Sorich MJ, Horowitz JD, Sorich W, et al. Cost-effectiveness of using CYP2C19 genotype to guide selection of clopidogrel or ticagrelor in Australia. Pharmacogenomics 2013; 14:2013-2021

29. Crespin DJ, Federspiel JJ, Biddle AK, et al. Ticagrelor versus genotypedriven antiplatelet therapy for secondary prevention after acute coronary syndrome: a cost-effectiveness analysis. Value Health 2011; 14:483-491

30. Mahoney EM, Wang K, Arnold SV, et al. Cost-effectiveness of prasugre versus clopidogrel in patients with acute coronary syndromes and planned percutaneous coronary intervention: results from the trial to assess improvement in therapeutic outcomes by optimizing platelet inhibition with prasugrel-thrombolysis in myocardial infarction TRITON-TIMI 38. Circulation 2010; 121:71-79

31. Schüpke S, Neumann FJ, Menichelli M, et al. Ticagrelor or prasugrel in patients with acute coronary syndromes. N Engl J Med 2019; 381:15241534 
Part III: Switching P2 $\mathbf{Y}_{12}$ inhibititors in the early phase after STEMI 


\section{Reloading when switching from ticagrelor or prasugrel to clopidogrel within 7 days after STEMI}

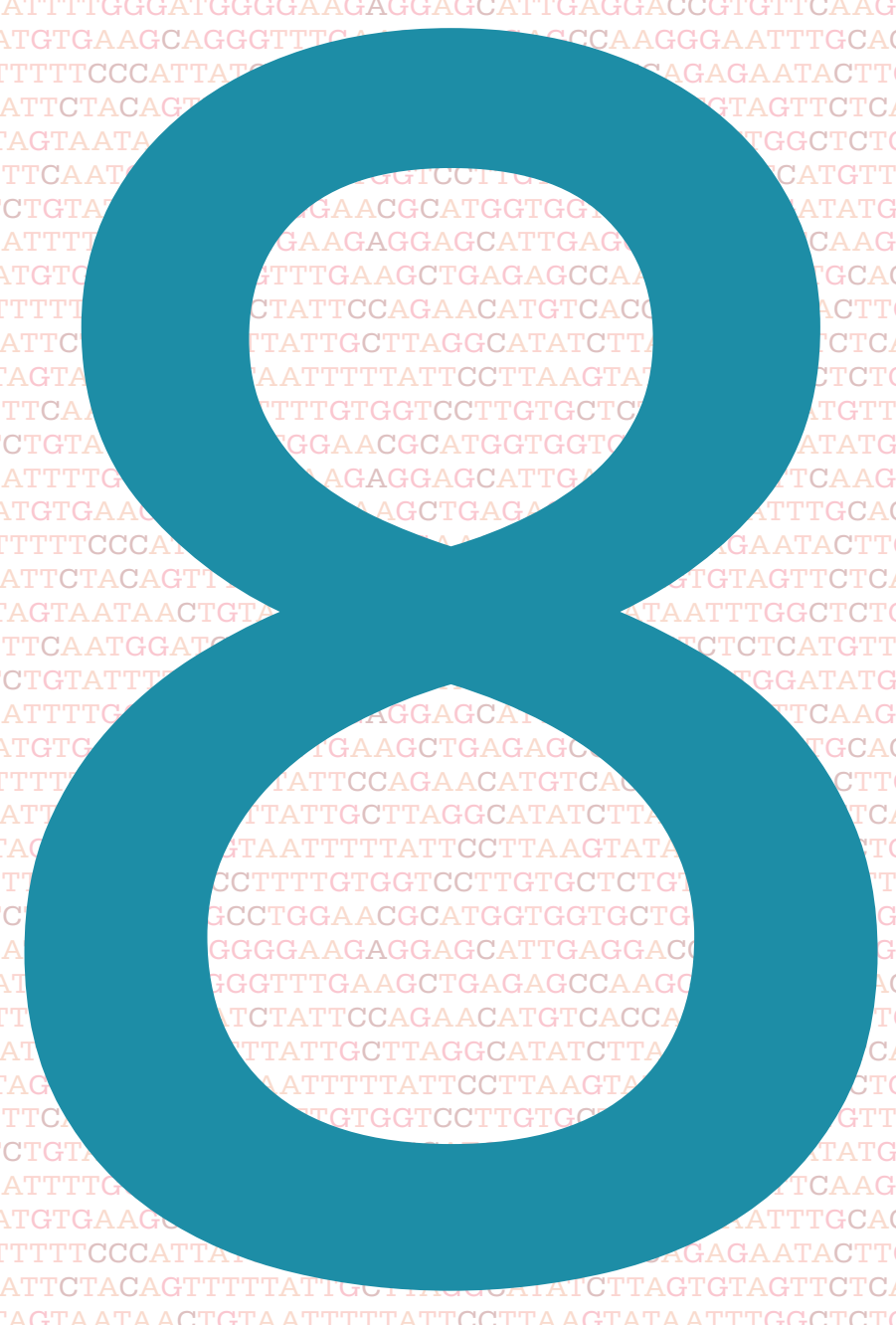

Daniel M.F. Claassens, Anne H. Tavenier, Renicus S. Hermanides, Gerrit J.A. Vos, Dario L. Hinrichs, Thomas 0. Bergmeijer, Arnoud W.J. van't Hof, Vera H.M. Deneer, Jurriën M. ten Berg

JACC: Cardiovascular Interventions 2020;13:663-665

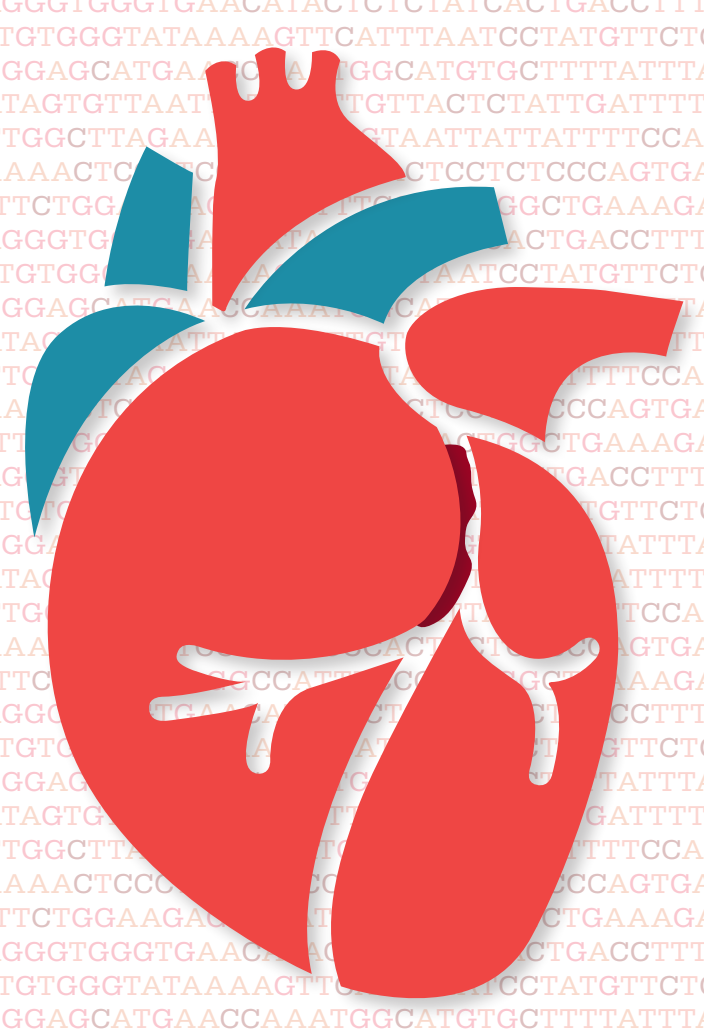


When switching from ticagrelor or prasugrel to clopidogrel early after myocardial infarction (MI), a loading dose is recommended [1, 2]. However, this recommendation is based solely on pharmacodynamics studies, whereas studies investigating the effects on clinical outcomes (i.e., stent thrombosis [ST] and bleeding) are lacking. In this pre-specified subanalysis of the POPular Genetics (Patient Outcome after Primary Percutaneous Coronary Intervention [PCI]) trial, we aimed to investigate the effect of loading with clopidogrel on clinical outcomes when switching from ticagrelor or prasugrel to clopidogrel early after MI.

Results of the POPular Genetics trial have been published recently [3] Noncarriers of loss-of-function alleles switching to clopidogrel within seven days after primary PCI and with a known loading dose status were included for this analysis. The primary thrombotic outcome consisted of cardiovascular death, MI, definite ST, and stroke. The primary bleeding outcome consisted of Platelet Inhibition and Patient Outcomes (PLATO) major and minor bleeding. Both outcomes were assessed at 7 days after switching. T $1 / 40$ was defined as the moment the first dose of clopidogrel was administered. Groups were compared using Student's t-test, chisquare test, or 1-way analysis of variance. The log-rank test was used to calculate $\mathrm{p}$ values. $\mathrm{p}$ Values below 0.05 were considered statistically significant.

Six hundred eighty-eight patients, of whom 172 received a loading dose, were suitable for this analysis. Seventy-one received clopidogrel $300 \mathrm{mg}$, and 101 received clopidogrel 600mg. Except for 7 patients who switched from prasugrel, the 681 other patients switched from ticagrelor to clopidogrel. Median time from primary PCI to switching was approximately $37 \mathrm{~h}$ [3]. Most patients receiving a loading dose when switching were included after publication of the European Society of Cardiology dual-antiplatelet therapy guideline in September 2017, whereas most patients that did not were included before that date. Probably because of this, there were some clear differences in procedural characteristics. Femoral access site $(34.1 \%$ vs. $15.3 \%)$, periprocedural use of glycoprotein IIb/IIIa receptor blocker $(46.6 \%$ vs. $29.8 \%)$, bivalirudin use $(5.9 \%$ vs. $1.2 \%)$, bare-metal stent use $(5.8 \%$ vs. $0 \%)$, and multivessel disease $(52.1 \%$ vs. $40.6 \%$ ) were more common in the patient group not receiving a loading dose. The thrombotic outcome did not occur within 7 days of switching in patients in the loading dose group, whereas it occurred in four patients in the no loading dose group $(0 \%$ vs. $0.8 \% ; \mathrm{p}=0.25)$ (Table 1$)$. Thrombotic events occurred at the day of switching (stroke and recurrent MI), at day 3 (ST) and at day 4 (coronary artery bypass surgery-related stroke). The bleeding outcome also did not occur in the loading dose group, whereas 3 patients in the no loading dose group experienced a bleeding event within 7 days after switching ( $0 \%$ vs. $\left.0.6 \% ; p^{1 / 4} 0.32\right)$, consisting of one coronary artery bypass grafting surgery-related PLATO major bleeding $\left(0.2 \% ; \mathrm{p}^{1 / 4}\right.$ $0.58)$ and two PLATO minor bleedings (0.4\%; $\left.\mathrm{p}^{1 / 4} 0.19\right)$. Bleeding events occurred 3 to 5 days after switching to clopidogrel.

In conclusion, we found that very low event rates irrespective of using a loading dose, while there were numerically less thrombotic and bleeding events in the loading dose group. These results give some support for using a clopidogrel loading dose. However, due to the low event rates and lack of randomization, this study should be considered as hypothesis generating, and definitive conclusions cannot be drawn. In addition, though clopidogrel may not provoke bleeding, these data cannot be extrapolated to prasugrel or ticagrelor loading doses. Furthermore, this study was performed in patients who were noncarriers of loss-of-function alleles, and results may therefore not be generalisable to carriers of lossof-function alleles who are at increased thrombotic risk and might benefit more from a loading dose. Despite these limitations, this is the first study reporting clinical outcomes in patients using a loading dose or not Furthermore, the decision to switch to clopidogrel was based on the trial protocol, whereas the decision to use a loading dose or not was homogenous among patients in each individual centre. These decisions were therefore less susceptible to bias.

\section{Table 1. Outcomes after using a loading dose versus no} loading dose when switching to clopidogrel.

\begin{tabular}{|l|l|l|l|}
\hline & $\begin{array}{l}\text { Loading dose } \\
(\mathrm{N}=172)\end{array}$ & $\begin{array}{l}\text { No loading dose } \\
(\mathrm{N}=516)\end{array}$ & P value \\
\hline $\begin{array}{l}\text { Thrombotic outcome } \\
\text { (cardiovascular death, MI, } \\
\text { definite ST, stroke) }\end{array}$ & $0(0)$ & $4(0.8)$ & 0.25 \\
\hline $\begin{array}{l}\text { Combined bleeding outcome } \\
\text { (PLATO major and minor } \\
\text { bleeding) }\end{array}$ & $0(0)$ & $3(0.6)$ & 0.32 \\
\hline PLATO major bleeding & $0(0)$ & $1(0.2)$ & 0.58 \\
\hline PLATO minor bleeding & $0(0)$ & $2(0.4)$ & 0.40 \\
\hline
\end{tabular}

Table 1. Values are $\mathrm{n}(\%)$ of patients. Outcomes are 7 days after switch.

$\mathrm{MI}=$ myocardial infarction; PLATO = platelet inhibition and patient outcomes $\mathrm{ST}=$ stent thrombosis 


\section{References}

1. Valgimigli M, Bueno H, Byrne RA, et al. 2017 ESC focused update on dual antiplatelet therapy in coronary artery disease developed in collaboration with EACTS. Eur Heart J 2018; 39:213-260

2. Angiolillo J, Rollini F, Storey RF, et al. International expert consensus on switching platelet P2Y12 Receptor-inhibiting Therapies. Circulation 2017; 136:1955-1975

3. Claassens DMF, Vos GJA, Bergmeijer TO, et al. A genotype-guided strategy for oral p2y12 inhibitors in primary PCI. N Engl J Med 2019; 381:1621-1631 


\section{General discussion}

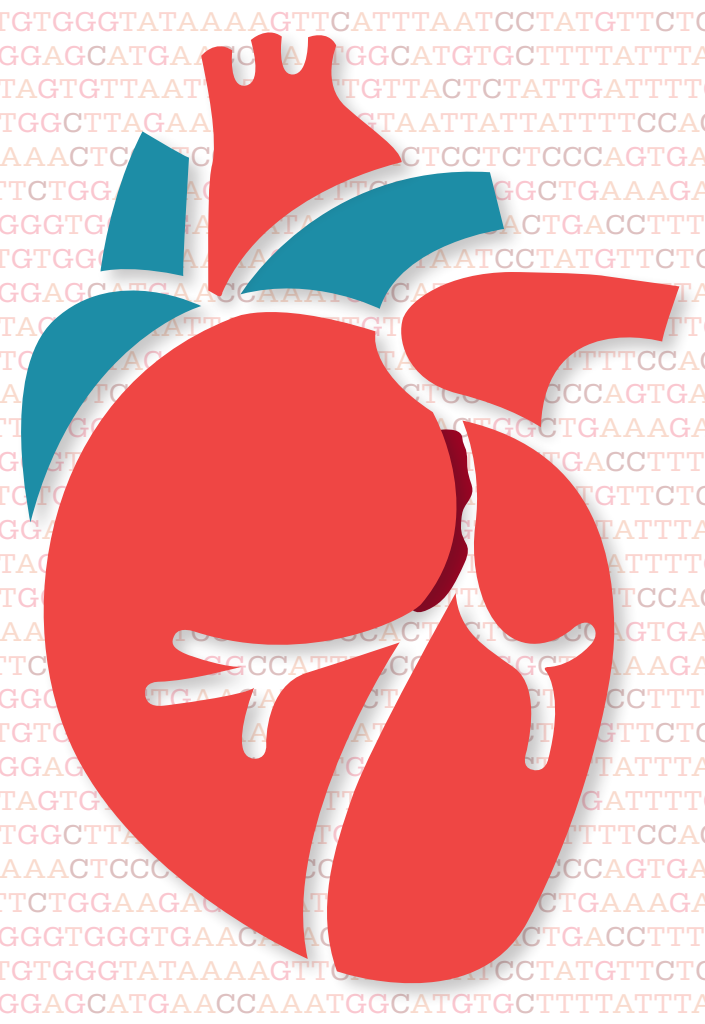


The most important gene that influences platelet reactivity in patients treated with clopidogrel is the CYP2C19 gene, which encodes the eponymous enzyme [1]. Since it was discovered that a standard dose of clopidogrel does not have the desired antiplatelet effect in all patients, alternative strategies for standard clopidogrel dosage have been investigated. However, the more potent $\mathrm{P}_{2} \mathrm{Y}_{12}$ inhibitors prasugrel and ticagrelor proved beneficial in reducing atherothrombotic events, they also led to an increase in bleedings [2,3]. Furthermore, a reduction in atherothrombotic events in the past decade due to newer stents and improved secondary prevention [4] marked a turning point where focus has shifted from only preventing atherothrombotic events to preventing bleeding events as well. This thesis focusses on using CYP2C19 genetic testing to de-escalate $\mathrm{P} 2 \mathrm{Y}_{12}$ inhibitor therapy. Most data is derived from the randomized POPular Genetics trial, which investigated whether a CYP2C19 genotype-guided strategy is feasible in clinical practice and whether it can help to reduce bleeding complication rates without increasing atherothrombotic event rates as compared to treatment with prasugrel or ticagrelor.

\section{De-escalation of $\mathrm{P} 2 \mathrm{Y}_{12}$ inhibitor therapy}

The first two chapters of this thesis form the introduction. Chapter 1 consists of a review regarding the rationale behind using CYP2C19 genetic testing and how it can be used to either escalate or de-escalate antiplatelet therapy, depending on what standard treatment is used [5]. When escalating, patients are treated with clopidogrel and switch to ticagrelor or prasugrel if they carry a CYP2C19 loss-of-function (LoF) allele. When deescalating, patients are treated with ticagrelor or prasugrel and switch to clopidogrel if they do not carry a loss-of-function allele. This thesis focusses is on de-escalation of antiplatelet therapy, which is introduced by a review in Chapter 2. This review describes three different methods to de-escalate antiplatelet therapy; unguided, platelet function testing (PFT)guided and genotype-guided. Results of the three corresponding randomized trials are presented [6]. All three methods share the same class $\mathrm{IIb}$ recommendation in the latest European Society of Cardiology (ESC) guideline for patients with an acute coronary syndrome without persistent ST-segment elevation to be used as an option to de-escalate treatment [7]. However, unguided de-escalation might not be the best option in all patients, as seen in observational data [8] and in patients carrying CYP2C19 LoF alleles. This was seen in the recent TAILOR PCI trial and in earlier trials like the TRITON TIMI 38 as well $[1,9]$. The review also provides pros and cons of using either genotyping or PFT and provides handles as to which patients could be selected for de-escalation and what strategy should be chosen when de-escalating to clopidogrel.

\section{Feasibility of using genetic testing in clinical practice}

In Chapter 3, the feasibility of using CYP2C19 genetic testing is studied by presenting descriptive data from two different cohorts [10]. The first cohort consists of 2556 patients from the POPular Risk Score (PRiS) project and the second cohort consists of 1038 patients from the POPular Genetics trial. The PRiS was a single-centre prospective registry that included consecutive patients that underwent non-urgent percutaneous coronary intervention (PCI) [10]. Blood samples were obtained from all participants and tested for the CYP2C19*2 and *3 loss-of-function (LoF) alleles three times daily by the on-site pharmacogenetic laboratory. The POPular Genetics trial was a multi-centre randomized controlled tria (RCT) that included ST-segment elevation myocardial infarction (STEMI) patients who underwent primary PCI [10]. Patients in the intervention arm were tested for the same LoF alleles as patients in the PRiS cohort. Testing was performed in a laboratory at the coordinating site using the same logistics as in the PRiS or by using a point-of-care (POC) system at each participating site. In the PRiS cohort, median time from blood collection to genotyping results was 4:04 hours. In the POPular Genetics, when on site genotyping was used, median time from randomization to genotyping results was 2:04 hours when using the lab at the coordinating site or 2:24 hours when using a POC system. In both studies, results were available within 24 hours in more than $90 \%$ of the patients. Though the POC system produced an inconclusive result in $8 \%$ of the tests, meaning the test has to be done again, it has some distinct advantages over laboratory-based tests since these require more training, equipment and infrastructure. In clinica practice, POC systems can provide even greater speeds than in a trial since written informed consent is not necessary anymore and tests can be performed all day long. With the one-hour turnaround time of the test, results in STEMI patients who undergo primary PCI could be available almost as soon as they leave the Cath lab.

\section{Clinical outcomes of CYP2C19 genetic testing}

The primary clinical outcomes of the POPular Genetics trial are reported in Chapter 4 [11]. In the POPular Genetics trial 2488 patients were randomized between standard treatment with ticagrelor or prasugre $(n=1246)$ or genotype-guided treatment $(n=1242)$. In the genotype-guided group, patients that carried a CYP $2 C 19 * 2$ or $* 3$ were treated with ticagrelor 
or prasugrel, while noncarriers were de-escalated to clopidogrel within 48 hours after PCI. Follow-up duration of the trial was 12 months after primary PCI. The genotype-guided strategy was non-inferior compared to standard treatment regarding the first co-primary outcome defined as allcause death, recurrent myocardial infarction, stent thrombosis, stroke and Platelet Inhibition and Patient Outcome (PLATO) major bleeding ( $\mathrm{HR}=$ $\left.0.87, \mathrm{P}_{\text {non-inferiority }}<0.001\right)$. The genotype-guided strategy was superior regarding the second co-primary outcome defined as PLATO major and minor bleeding ( $\mathrm{HR} 0.78, \mathrm{P}=0.04$ ). This was mainly driven by a reduction in PLATO minor bleedings. Based on these results, using a genotypeguided strategy for all STEMI patients that undergo PCI could be beneficial.

Critics of the strategy argue that in a post-hoc analysis of the PLATO trial, which included 7080 patients (recruited between 2006 and 2008), ticagrelor proved to be superior compared to clopidogrel irrespective of CYP2C19 genotype [12]. However, regarding the thrombotic outcome, ticagrelor was not superior compared to clopidogrel in noncarriers of a LoF allele $(\mathrm{HR}=0.86, \mathrm{P}=0.06)$. Since then, event rates have decreased considerably due to newer stents and improved secondary prevention. For instance, in the subgroup analysis of PLATO which included STEMI patients intended for reperfusion with primary PCI, a thrombotic event rate (consisting of cardiovascular death, MI and stroke) of $9.4 \%$ was found in the ticagrelor group [13]. That is almost three times the rate seen in POPular Genetics and other recently published trials like TAILOR PCI and TROPICAL ACS $[9,14]$. Therefore, a similar analysis is presented in Chapter 5 [15]. It included 2429 patients, 821 noncarriers of LoF alleles treated with clopidogrel and 1608 patients treated with ticagrelor or prasugrel irrespective of their genotype. It found similar rates of atherothrombotic events (cardiovascular death, myocardial infarction, stent thrombosis, stroke) in both groups $(\mathrm{HR}=1.14, \mathrm{P}=0.62)$ and a significant reduction in (mostly minor) bleedings in clopidogrel treated patients $(\mathrm{HR}=0.74, \mathrm{P}=0.03)$. The on-treatment analysis found similar results with no differences in atherothrombotic events $\mathrm{HR}=0.98, \mathrm{P}=0.94)$ and a similar reduction in bleeding events $(\mathrm{HR}=0.69, \mathrm{P}=0.008)$ in clopidogrel treated patients. The results of these analyses in combination with the main trial results suggest the strategy of de-escalation is safe and could benefit patient outcomes.

\section{Elderly patients}

Higher age is an independent predictor of high on-treatment platelet reactivity when being treated with clopidogrel [16]. This is why Chapter
6 reports outcomes of $C Y P 2 C 19$ genetic testing in patients aged 70 and older. In the POPular Age trial, which randomized non-STEMI and unstable angina patients aged 70 years and older to receive either ticagrelor/prasugrel or clopidogrel in combination with aspirin during one year [17], CYP2C19 genetic testing was performed at the end of the trial Data of these patients was combined with data from patients in the POPular Genetics trial aged 70 years and older to form the presented analysis. Furthermore, it contains subgroup analysis of the POPular Age and POPular Genetics separately. In the combined analysis, 1002 patients were included, 402 noncarriers of LoF alleles treated with clopidogrel and 600 patients treated with ticagrelor or prasugrel. In this small subgroup, results were comparable to the POPular Genetics with no difference in atherothrombotic event rates $(\mathrm{HR}=1.00, \mathrm{P}=0.98)$ and less bleeding events in clopidogrel treated patients $(\mathrm{HR}=0.83, \mathrm{P}=0.23)$, though this difference was not statistically significant. In addition, in the POPular Genetics subgroup analysis, age was not a significant interaction variable [11], suggesting results are generalizable across all ages. In the POPular Age trial, clopidogrel treated patients had lower bleeding event rates $(\mathrm{HR}=$ $0.71,95 \%$ CI 0.54-0.94) and similar atherothrombotic event rates (HR = $0.92,95 \%$ CI $0.65-1.34$ ) as ticagrelor or prasugrel treated patients, while clopidogrel treated patients were not prospectively tested for the CYP2C19 gene [17]. Therefore, one could question whether genetic testing provides any additional value in elderly patients. However, these results are in contradiction to results from the Bremen STEMI registry, TAILOR PCI trial and a genetic sub analysis of the TRITON TIMI 38 trial, which suggest clopidogrel treatment, is not the best option for all (elderly) patients and that genetic testing can help select patients that do benefit $[1,9,18]$.

\section{CYP2C19*17 allele}

While most of this thesis focusses on the CYP2C19 LoF alleles, some argue that the $* 17$ allele has a gain-of-function effect. Clopidogrel treated carriers of this allele would have less chance of an atherothrombotic event and a higher chance of a bleeding event than noncarriers of this allele. In addition to the previous sub analysis, Chapter 5 also focusses on the existence of this effect. The subgroup analysis included clopidogrel treated noncarriers of LoF and compared 312 carriers of *17 alleles to 420 CYP2C19*1/*1 patients. It found no evidence for a gain-of-function effect on clinical outcomes with similar rates of atherothrombotic events $(\mathrm{HR}=$ $0.95, \mathrm{P}=0.90)$ and a non-significant lower bleeding event rate $(\mathrm{HR}=0.74$, $\mathrm{P}=0.21)$ in $* 17$ carriers. These results are in accordance with a study by Lewis et al., which found that the $* 2$ and $* 17$ alleles were in complete 
linkage disequilibrium [19]. This means the two alleles do not exist on the same chromosome. The gain-of-function effect of the $* 17$ allele was mostly, if not entirely, derived from the non-existence of the CYP2C19*2 variant.

\section{Cost-effectiveness}

With aging populations and advances in diagnostics and treatments of diseases, healthcare costs are rapidly increasing across the globe. We now live in an era where medical innovations and its associated costs have reached or even surpassed the economic capabilities of many modern countries. Therefore, the importance of health economic analyses has increased in the past decades. Chapter 7 presents a cost-effectiveness analysis of implementing a CYP2C19 genotype-guided strategy based on the POPular Genetics trial. While earlier studies have already shown this to be a cost-effective strategy [20-22], these studies based their assumptions on trials that did not specifically investigate a genotypeguided strategy. The current analysis compared two cohorts of 1000 patients with STEMI undergoing primary PCI. The first cohort was treated using a CYP2C19 genotype-guided strategy, while the second was treated with ticagrelor or prasugrel. After a one-year decision tree based on the POPular Genetics trial, all patients entered a life-long Markov model to simulate costs and effects. In the base-case analysis, a genotype-guided strategy resulted in 8.98 quality-adjusted life-years (QALYs) gained and $€ 725,550.69$ in cost-savings (dominant) per 1000 patients. In scenario analyses simulating different time horizons, different discount rates, equal drug prices for all $\mathrm{P}_{2} \mathrm{Y}_{12}$ inhibitors (simulating the availability of generic ticagrelor and prasugrel) and an equal distribution of atherothrombotic events at the beginning of the Markov model, the genotype-guided strategy remained dominant with cost-savings and increased QALYs. These results suggest a CYP2C19 genotype-guided strategy does not only improve patient outcomes, but also reduces healthcare costs.

\section{Switching P2Y12 inhibitors in the early phase after STEMI}

Clopidogrel, ticagrelor and prasugrel have different pharmacodynamics and kinetics. When switching between these drugs, especially in the early phase after myocardial infarction, it is important to have a sufficient reduction in platelet reactivity, while trying to keep the bleeding risk as low as possible. Therefore, the ESC has published a focused update on dual antiplatelet therapy, which provides recommendations on how to switch between the different drugs [23]. It recommends using a 600mg loading dose of clopidogrel 24 hours after last administration of ticagrelor or prasugrel. However, this recommendation is based solely on pharmacodynamic studies that present only limited results on clinical outcomes. Therefore, Chapter 8 reports on outcomes when switching from ticagrelor (and in a few cases prasugrel) to clopidogrel within one week after primary PCI [24]. This was done without a loading dose in 516 patients (mostly included earlier in the trial), while a loading dose of either $300 \mathrm{mg}$ or, in most cases $600 \mathrm{mg}$, was used in 172 patients. In the group of patients that received a loading dose of clopidogrel, there were no atherothrombotic or bleeding events in the 7 days after switching, while in the patient group that received no loading dose, four patients experienced an atherothrombotic and three patients experienced a bleeding event. The non-randomized nature of this study and the small number of events restrict drawing any strong conclusions, but using a new loading dose does no seem to increase the risk of bleeding and might help to reduce atherothrombotic events as compared to not using a loading dose.

\section{Future perspective}

While in cancer treatment, genetic analysis of different types of cancer has already found widespread implementation to guide treatment, in the field of cardiovascular medicine, personalised therapies still have a limited role in clinical practice. For a widespread implementation of personalised antiplatelet strategies, several requirements have to be met; 1) it should improve clinical outcomes, 2) results have to be consistent and easy to interpret, 3) the test should be easy to perform, 4) results should be available within a reasonable amount of time and 5) costs have to be reimbursed.

The first requirement has partially been met. The first studies prospectively investigating a CYP2C19 genotype-guided approach were published almost a decade ago and there has been an increasing interest amongs cardiologists in the strategy since then. While the POPular Genetics trial demonstrated a clear benefit in reducing bleeding event rates, it was not powered to detect differences in ischemic events. Probably, this is the reason that the latest NSTEMI guideline from the ESC only gives the strategy a class IIb recommendation (may be considered). For a higher recommendation in the guidelines, more data is needed. This could either be provided by additional, larger RCTs or by providing more registry data of the strategy in clinical practice. However, large RCTs are impossible to execute without financial support (mostly provided by the pharmaceutical industry) and currently no large trials are expected in the near future. 
With a paucity of very large RCTs, meta-analysis could be considered the next best evidence. Recently, a meta-analysis by Pereira et al. was published. It included 15,949 patients ( $98 \%$ with acute coronary syndrome, $77 \%$ treated with $\mathrm{PCI}$ ) and compared clopidogrel to ticagrelor or prasugre separately in noncarriers of CYP2C19 loss-of-function alleles and in carriers of loss-of-function alleles [25]. They found that ticagrelor and prasugrel compared with clopidogrel resulted in a significant reduction in atherothrombotic events in CYP2C19 loss-of-function carriers (Relative risk $0.70,95 \% \mathrm{CI}, 0.59-0.83$ ), but not in noncarriers (RR $1.0,95 \% \mathrm{CI}$ $0.80-1.25)$. The test for interaction was statistically significant $(\mathrm{p}=$ 0.013 ), suggesting this effect is caused by carrying CYP2C19 loss-offunction alleles. Therefore, this meta-analysis, which compares the $\mathrm{P} 2 \mathrm{Y}_{12}$ inhibitors directly, demonstrated clopidogrel, in noncarriers of CYP2C19 loss-of-function alleles, is as effective as ticagrelor and prasugrel. Thus providing more, though indirect, evidence that a genotype-guided strategy can be used. Another large meta-analysis, by Galli et al., was also published recently [26]. It compared guided therapy (both de-escalation and escalation strategies using genotyping or PFT) to standard treatment and found that guided therapy reduced major adverse cardiac events (RR $0.78,95 \% \mathrm{CI}, 0.63-0.95)$ and a trend towards a reduction in bleedings (RR 0.88, 95\% CI 0.77 - 1.01). Comparing escalation and de-escalation strategies separately, on the one hand it found that a guided escalation strategy compared to standard clopidogrel treatment resulted in reductions in atherothrombotic outcomes without increasing bleeding event rates. On the other hand, it found that a guided de-escalation strategy compared to standard ticagrelor or prasugrel treatment resulted in a reduction in bleedings (driven by minor bleedings), without any differences in atherothrombotic outcomes. Findings of these meta-analyses might provide a reason for a higher recommendation in future guidelines.

The second requirement has been met by using genetic testing. A genotype-guided strategy has distinct advantages over, for instance, a PFT guided strategy. PFT requires a patient to already be on clopidogrel for a considerable amount of time before it can be tested. Additionally, many factors influence PFT and there is a large variability in results between different PFT tests. Genetic testing does not have these disadvantages as long as there is no contamination of the blood sample or buccal swab.

POC systems like the one from Spartan Bioscience are easy to use and require only limited training. Also, a buccal swab provides almost no burden for the patient. However, during the POPular Genetics trial, almost $10 \%$ of samples returned inconclusive and I noticed differences in error rates from the tests between different centres. This could be due to incorrectly obtaining the buccal swab or incorrectly preparing the samples before testing. The inconclusive rate should be lower than $10 \%$ if it is to be widely used. This could be achieved by better training, or the inconclusive rate may improve simply due to more experience. Perhaps, newer techniques become available which reduce the chance of inconclusive results. Additionally, the buccal swabs cannot be stored in standard freezers, since they require lower temperatures (between $-20^{\circ}$ and $-80^{\circ}$ Celsius). For the test to be more accessible, this could also be improved upon. Therefore, despite a few shortcoming that have to be improved in the future, it is my opinion that the third requirement has also been met. Furthermore, laboratory based tests can also be used without additional burden for the patient if the blood sample is taken at the time of coronary angiography. However, the required expertise, and infrastructure is not available in all hospitals.

The fourth requirement has also been met. Both data from the POPular Genetics trial, PRiS and data from other groups investigating a genotypeguided strategy [27] have demonstrated that results can be available within hours. A POC system could be used 24 hours daily by personnel at the Cath lab or coronary care unit, while with a dedicated system, as seen in the St. Antonius hospital, even laboratory-based tests can be used with sufficient speed.

The fifth requirement, as of now, seems to be the largest obstacle for many hospitals. Implementing a genotype-guided strategy is associated with significant costs for hospitals. Cost-savings, however, do not end up in the hospital, but with the insurance companies or governments that pay for healthcare. In the United States, both Medicare and Medicaid already provide reimbursement, but this is not the case in the Netherlands and other European countries. The availability of cost-effectiveness analyses might help to convince policymakers in the government and insurance companies to change their strategy. While more data from meta-analyses and registries demonstrating a beneficial effect on hard clinical outcomes could also help tip the skill.

While this thesis focusses on using a genotype-guided strategy to personalize and de-escalate antiplatelet therapy after myocardial infarction, other methods of de-escalation are also investigated. Results from several RCTs investigating different options, like de-escalating from normal dose ticagrelor ( $90 \mathrm{mg}$ twice daily) or prasugrel (10mg once daily) to low dose ticagrelor (60mg twice daily) or prasugrel ( $5 \mathrm{mg}$ once daily) in combination with aspirin have been published in recent years. 
Simultaneously, other trials investigated strategies where aspirin was discontinued after one to three months after PCI and thus patients were treated with ticagrelor or prasugrel monotherapy. Since none of these strategies have been compared head-to-head or to genotype or PFT-guided strategies, it is currently unknown, which provides the best outcomes for patients and what strategy is most practical. Only time will tell, but improvements in clinical outcomes are sure to continue.

\section{References}

1. Mega JL, Close SL, Wiviott SD, et al. Cytochrome $\mathrm{p}-450$ polymorphisms and response to clopidogrel. N Engl J Med 2009; 360:354-362

2. Wallentin L, Becker RC, Budaj A, et al. Ticagrelor versus clopidogrel in patients with acute coronary syndromes. N Engl J Med 2009; 361:1045-1057

3. Wiviott SD, Braunwald E, McCabe CH, et al. Prasugrel versus clopidogrel in patients with acute coronary syndromes. N Engl J Med 2007; 357:20012015

4. Puymirat E, Cayla G, Cottin Y et al. Twenty-year trends in profile, management and outcomes of patients with ST-segment elevation myocardial infarction according to use of reperfusion therapy: data from the FASTMI program 1995-2015. Am Heart J. 2019; 214:97-106

5. Claassens DMF, Ten Berg JM. Genotype-guided treatment of oral P2Y12 inhibitors: where do we stand? Pharmacogenomics 2020; 21:83-86

6. Claassens DMF, Sibbing D. De-Escalation of Antiplatelet Treatment in Patients with Myocardial Infarction Who Underwent Percutaneous Coronary Intervention: A Review of the Current Literature. J Clin Med 2020; 9:2983

7. Collet JP, Thiele H, Barbato E, et al. 2020 ESC Guidelines for the management of acute coronary syndromes in patients presenting without persistent ST-segment elevation. Eur Heart J 2021; 42:1289-1367

8. De Luca L, D'Ascenzo F, Tarantini G, et al. Incidence and Outcome of Switching of Oral Platelet P2Y12 Receptor Inhibitors in Patients with Acute Coronary Syndromes Undergoing Percutaneous Coronary Intervention: The SCOPE Registry. EuroIntervention 2017; 13:459-466

9. Pereira NL, Farkouh ME, So D, et al. Effect of Genotype-Guided Oral P2Y12 Inhibitor Selection vs Conventional Clopidogrel Therapy on Ischemic Outcomes After Percutaneous Coronary Intervention: The TAILOR-PCI Randomized Clinical trial. JAMA 2020; 324:761-771

10. Bergmeijer TO, Vos GJA, Claassens DMF, et al. Feasibility and implementation of CYP2C19 genotyping in patients using antiplatele therapy. Pharmacogenomics 2018; 19:621-628

11. Claassens DMF, Vos GJA, Bergmeijer TO, A Genotype-guided strategy for oral P2Y12 inhibitors in primary PCI. N Engl J Med 2019; 381:1621-1631

12. Wallentin L, James S, Storey RF, et al. Effect of CYP2C19 and ABCB1 single nucleotide polymorphisms on outcomes of treatment with ticagrelor versus clopidogrel for acute coronary syndromes: a genetic substudy of the PLATO trial. Lancet 2010; 376:1320-1328

13. Steg PG, James S, Harrington RA, et al. Ticagrelor versus clopidogrel in patients Ticagrelor versus clopidogrel in patients with ST-elevation acute coronary syndromes intended for reperfusion with primary percutaneous coronary intervention: A Platelet Inhibition and Patient Outcomes (PLATO) trial subgroup analysis. Circulation 2010; 122:2131-214 
14. Sibbing D, Gross L, Trenk D, et al. Age and outcomes following guided deescalation of antiplatelet treatment in acute coronary syndrome patients undergoing percutaneous coronary intervention: Results from the randomized TROPICAL-ACS trial. Eur Heart J 2018; 39:2749-2758

15. Claassens DMF, Bergmeijer TO, Vos GJA, et al. Clopidogrel versus ticagrelor or prasugrel after primary percutaneous coronary intervention according to CYP2C19 genotype. Circ Cardiovasc Interv 2021; 14:e009434

16. Silvain J, Cayla G, Hulot JS, et al. High on-thienopyridine platelet reactivity in elderly coronary patients: the SENIOR-PLATELET study. Eur Heart 2012; 33:1241-1249

17. Gimbel M, Qaderdan K, Willemsen L, et al. Clopidogrel versus ticagrelor or prasugrel in patients aged 70 years or older with non-ST-elevation acute coronary syndrome (POPular AGE): the randomised, open-label, noninferiority trial. Lancet 2020; 395:1374-1381

18. Schmucker J, Fach A, Mata Marin LA, et al. Efficacy and Safety of Ticagrelor in Comparison to Clopidogrel in Elderly Patients With STSegment-Elevation Myocardial Infarctions. J Am Heart Assoc 2019; 8:e012530

19. Lewis JP, Stephens SH, Horenstein RB et al. The CYP2C19*17 variant is not independently associated with clopidogrel response. J Thromb Haemost $2013 ; 11: 1640-1646$

20. Lala A, Berger JS, Sharma G, et al. Genetic testing in patients with acute coronary syndrome undergoing percutaneous coronary intervention: a costeffectiveness analysis. J Thromb Haemost 2013; 11:81-91

21. Wang Y, Yan BP, Liew D, et al. Cost-effectiveness of cytochrome P450 $2 \mathrm{C} 19 * 2$ genotype-guided selection of clopidogrel or ticagrelor in Chinese patients with acute coronary syndrome. Pharmacogenomics J 2018; 18:113120

22. Limdi NA, Cavallari LH, Lee CR, et al. Cost-effectiveness of CYP2C19guided antiplatelet therapy in patients with acute coronary syndrome and percutaneous coronary intervention informed by real-world data. Pharmacogenomics J 2020; 20:724-735

23. Valgimigli M, Bueno H, Byrne RA, et al. 2017 ESC focused update on dua antiplatelet therapy in coronary artery disease developed in collaboration with EACTS. Eur Heart J 2018; 39:213-260.

24. Claassens DMF, Tavenier AH, Hermanides RS, et al. Reloading when switching from ticagrelor or prasugrel to clopidogrel within 7 days after STEMI. JACC Cardiovasc Interv 2020; 13:663-665

25. Pereira NL, Rihal C, Lennon R, et al. Effect of CYP2C19 genotype on ischemic outcomes during oral P2Y12 inhibitor therapy. JACC Cardiovasc Interv 2021; 14:739-750

26. Galli M, Benenati S, Capodanno D, et al. Guided versus standard antiplatelet therapy in patients undergoing percutaneous coronary intervention: a systematic review and meta-analysis. The Lancet 2021; 397:1470-1483
27. Al-Rubaish AM, Al-Muhanna FA, Alshehri AM, et al. Bedside testing of CYP2C19 gene for treatment of patients with PCI with antiplatelet therapy. BMC Cardiovasc Disord 2020; 20:268 
Nederlandse samenvatting

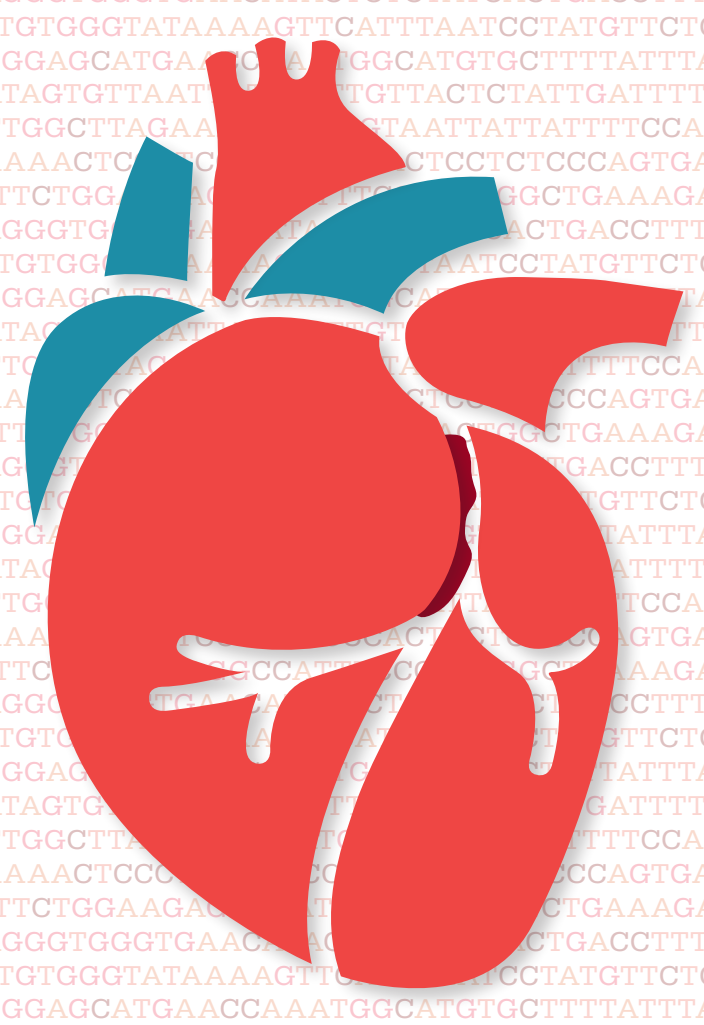


Plaatjesaggregatie remmers worden voorgeschreven aan patiënten met een myocardinfarct om de kans op een recidief te verkleinen. Patiënten worden behandeld met duale antiplaatjes therapie, bestaande uit aspirine en een $\mathrm{P}_{2} \mathrm{Y}_{12}$ remmer. De Europese en Amerikaanse richtlijnen geven daarbij de voorkeur aan de sterkere $\mathrm{P}_{2} \mathrm{Y}_{12}$ remmers ticagrelor of prasugrel in plaats van clopidogrel, omdat mensen die behandeld worden met deze sterkere middelen minder kans hebben op ischemische events zoals stent trombose, myocardinfarcten, herseninfarcten en overlijden. Behandeling met deze sterkere $\mathrm{P} 2 \mathrm{Y}_{12}$ remmers geeft echter ook een grotere kans op bloedingen. Ook bloedingen kunnen ernstige gevolgen hebben en zijn geassocieerd met slechtere uitkomsten, zoals bijvoorbeeld overlijden. Het is daarom belangrijk om deze te voorkomen.

Clopidogrel is een pro-drug welke gemetaboliseerd wordt door cytochroom P450 iso-enzymen om actief te worden. Het belangrijkste enzym in dit proces is het CYP2C19 enzym. Dit enzym wordt gecodeerd door het CYP2C19 gen. Ongeveer 1/3 van de Kaukasische bevolking draagt allelen van het gen waardoor de metabolisering minder goed verloopt, zogenaamde verlies-van-functie allelen. Het $* 2$ allel en in mindere mate het $* 3$ allel zijn hiervan verreweg de meest voorkomende allelen. In dit proefschrift is onderzocht of een strategie waarbij gebruik wordt gemaakt van een genetische test voor het CYP2C19 gen leidde to minder bloedingen zonder dat er meer ischemische events waren. Dit werd vergeleken met de uitkomsten van een standaardbehandeling waarbij iedereen behandeld werd met ticagrelor of prasugrel. In de genotypegestuurde strategie behandelden we patiënten die drager waren van verliesvan-functie allelen met ticagrelor of prasugrel, terwijl mensen die geen drager waren behandeld werden met clopidogrel. Daarnaast is gekeken of deze strategie praktisch haalbaar is in de praktijk en of deze strategie kosteneffectief is. Tenslotte is ook gekeken of er een nieuwe oplaaddosis van clopidogrel moet worden gegeven als er gede-escaleerd wordt van ticagrelor of prasugrel naar clopidogrel.

De inleiding van dit proefschrift bestaat uit hoofdstuk 1 en hoofdstuk 2. Hoofdstuk 1 bevat een review waarin werd ingegaan op de grondgedachte achter het gebruik van CYP2C19 genetische testen. Het beschrijft zowel onderzoek naar het escaleren van antiplaatjes therapie (switchen van clopidogrel naar ticagrelor of prasugrel wanneer iemand drager is van verlies-van-functie allelen) als naar het de-escaleren van antiplaatjes therapie (switchen van ticagrelor of prasugrel naar clopidogrel als iemand geen drager is van verlies-van-functie allelen). In hoofdstuk 2 werd in een review specifieker ingegaan op het de-escaleren. Dit kan op drie verschillende manieren; 1) zonder te testen; 2) door gebruik te maken van plaatjes functie testen; en 3) door gebruik te maken van genetische testen. Op basis van drie verschillende gerandomiseerde onderzoeken hebben al deze methoden dezelfde klasse IIb indicatie gekregen (er kan overwogen worden deze strategie te gebruiken), in de laatste European Society of Cardiology (ESC) richtlijn voor patiënten met een acuut coronair syndroom zonder persisterende ST-segment elevaties. Echter, op basis van enkele andere onderzoeken, zoals de recent gepubliceerde TAILOR PCI en de oudere TRITON TIMI 38 studie, lijkt de-escaleren zonder gebruik te maken van een test niet de beste optie bij alle patiënten, met name bij dragers van CYP2C19 verlies-van-functie allelen. Verder beschrijft de review de voor- en nadelen van een genetische test ten opzichte van een plaatjesfunctie test en op welke strategie gevolgd moet worden als er gedeescaleerd wordt.

\section{Haalbaarheid van het implementeren van CYP2C19 genetisch testen}

In hoofdstuk 3 werd de haalbaarheid van het implementeren van CYP2C19 testen in de praktijk beschouwd, op basis van twee verschillende cohorten. Het ene cohort bestond uit 2556 patiënten uit de POPular Risk Score (PRiS) studie en het andere cohort bestond uit 1038 patiënten uit de POPular Genetics studie. De PRiS was een prospectieve registratie-studie uitgevoerd in één centrum en includeerde patiënten die een electieve percutane coronaire interventie (PCI) ondergingen. Bij alle patiënten werd bloed afgenomen en dit werd drie keer per dag in het farmacogenetisch lab getest op de aanwezigheid van de CYP2C19*2 en *3 verlies-van-functie allelen. De POPular Genetics studie was een gerandomiseerde studie en includeerde patiënten met een ST-segment elevatie myocardinfarct die een primaire PCI ondergingen. In de interventiegroep werden patiënten op dezelfde allelen getest als in de PRiS studie. Testen werden in het coördinerende centrum volgens dezelfde methode uitgevoerd als in de PRiS studie of patiënten werden in het eigen ziekenhuis getest door gebruik te maken van een point-of-care (POC) systeem. In de PRiS was de mediane tijd van bloedafname tot de uitslag ruim 4 uur. In de POPular Genetics was de tijd van randomisatie tot het bekend worden van het CYP2C19 genotype iets meer dan 2 uur wanneer patiënten in het eigen ziekenhuis getest werden, zowel bij tests via het lab als via het POC-systeem. In beide studies 
was bij meer dan $90 \%$ van de patiënten de uitslag binnen 24 uur bekend. Bij het POC-systeem was ongeveer $8 \%$ van de uitslagen niet conclusief, waardoor de test opnieuw gedaan moest worden. Toch heeft het gebruik van POC-systemen voordelen doordat er minder training, apparatuur en infrastructuur vereist is. Daarnaast kunnen deze testen 24 uur per dag worden gebruikt, bijvoorbeeld door verpleegkundige of personaal op de hartkatheterisatie kamers. Als ze in de klinische praktijk worden gebruikt kan de uitslag al binnen 1 uur bekend zijn. Medicatie kan dan al volgens een CYP2C19 genotype-gestuurde strategie worden aangepast zodra een patiënt de hartkatheterisatie kamer verlaat.

\section{Klinische uitkomsten bij het gebruik van een CYP2C19 genotype-} gestuurde strategie

De primaire klinische uitkomsten van de POPular Genetics studie werden beschreven in hoofdstuk 4. In de POPular Genetics studie werden 2488 patiënten gerandomiseerd naar een standaardbehandeling met ticagrelor of prasugrel $(n=1246)$ of naar een genotype-gestuurde strategie $(n=1242)$. In de genotype-gestuurde strategie werden patiënten die dragers waren van een $C Y P 2 C 19 * 2$ of $* 3$ allel behandeld met ticagrelor of prasugrel en patiënten die geen dragers waren met clopidogrel. Deze verandering in medicatie gebeurde binnen 48 uur na de primaire PCI en follow-up van het onderzoek liep tot 12 maanden na de primaire PCI. De genotype-gestuurde groep was non-inferieur ten opzichte van de standaardbehandeling met betrekking tot de eerste co-primaire uitkomst bestaande uit overlijden, myocardinfarct, stent trombose, cerebrovasculair accident (CVA) en grote bloedingen gedefinieerd volgens Platelet Inhibition and Patient Outcome (PLATO) (HR 0.87, $\left.\mathrm{P}_{\text {non-inferieur }}<0.001\right)$. De genotype-gestuurde strategie was superieur met betrekking tot de tweede co-primaire uitkomst bestaande uit PLATO grote en kleine bloedingen $(\mathrm{HR}=0.78, \mathrm{P}=0.04)$ Dit werd voornamelijk veroorzaakt door een vermindering van het aantal kleine bloedingen.

In hoofdstuk 5 werden twee verschillende patiëntengroepen met elkaar vergeleken, namelijk patiënten die geen drager zijn van verlies-van-functie allelen behandeld met clopidogrel en patiënten die behandeld zijn met ticagrelor of prasugrel. Er werd gekeken naar dezelfde eindpunten als genoemd in de POPular Genetics studie. Er werden 2429 patiënten geïncludeerd, waarvan er 821 werden behandeld met clopidogrel en 1608 met ticagrelor of prasugrel. Er werd geen verschil gezien in het aantal ischemische uitkomsten (cardiovasculair overlijden, myocardinfarct, stent trombose en $\mathrm{CVA}$ ) tussen beide groepen $(\mathrm{HR}=1.14, \mathrm{P}=0.62)$ en er was een significante vermindering van het aantal (meestal kleine) bloedingen $(\mathrm{HR}=0.74, \mathrm{P}=0.03)$.

Omdat leeftijd een onafhankelijke voorspeller is voor onvoldoende plaatjesremming bij patiënten die behandeld worden met clopidogrel, is in hoofdstuk 6 specifiek gekeken naar oudere patiënten. Een deel van de patiënten uit deze analyse komt uit de POPular Age studie, waarin patiënten van 70 jaar en ouder met een niet-ST-segment elevatie myocardinfarct of instabiele angina pectoris werden geïncludeerd. Zij werden gerandomiseerd tussen clopidogrel enerzijds of ticagrelor of prasugrel anderzijds. Vooraf werd geen genetische test verricht, maar bij de patiënten waar bloed van beschikbaar was werd aan het einde van he onderzoek getest op de CYP2C19*2 en *3 verlies-van-functie allelen. Deze patiënten werden gecombineerd met de patiënten van 70 jaar en ouder uit de POPular Genetics studie en net als in hoofdstuk 5 werden patiënten zonder verlies-van-functie allelen behandeld met clopidogrel vergeleken met patiënten behandeld met ticagrelor. In totaal waren er 991 patiënten, 401 behandeld met clopidogrel en 590 met ticagrelor. In deze kleinere groep met ouderen waren er vergelijkbare resultaten als bij de gehele groep, echter was het verschil in bloedingen niet statistisch significant (ischemische uitkomsten $\mathrm{HR}=1.00, \mathrm{P}=0.98$, bloedingen $\mathrm{HR}=0.83, \mathrm{P}=$ $0.23)$. Tevens gaf leeftijd in de subgroep analyse van de POPular Genetics geen significante interactie. Daarom lijkt ook bij ouderen het gebruik van een CYP2C19 genotype strategie voordelen te hebben.

\section{Heeft het CYP2C19*17 allel een versterkende werking}

Hoewel het grootste deel van dit proefschrift zich focust op verlies-vanfunctie allelen, zijn er ook onderzoekers die beargumenteren dat het veel voorkomende *17 allel zorgt voor een sterkere werking van clopidogrel. In hoofdstuk 5 werd daarom ook bekeken of dit effect zich zou uiten in klinische uitkomsten. In deze subgroep analyse zijn 732 patiënten geïncludeerd die behandeld waren met clopidogrel en die geen drager waren van een verlies-van-functie allel. Hiervan waren er 312 patiënten drager van één of twee *17 allelen en 420 patiënten hadden het $* 1 / * 1$ genotype. Er werden geen aanwijzingen gevonden voor het bestaan van een versterkende werking van het $* 17$ allel. Er werd een vergelijkbaar aantal ischemische uitkomsten gezien in beide groepen $(\mathrm{HR}=0.95, \mathrm{P}=$ 
$0.90)$ en een niet significant lager aantal bloedingen in $* 17$ dragers $(\mathrm{HR}=$ $0.74, \mathrm{P}=0.21)$. Als er een versterkende werking zou zijn, zou juist verwacht worden dat er meer bloedingen zijn en minder ischemische uitkomsten.

\section{Kosteneffectiviteit van het implementeren van een CYP2C19 genotype-gestuurde strategie}

Hoofdstuk 7 behandelde de kosteneffectiviteit van het invoeren van een CYP2C19 genotype-gestuurde strategie vergeleken met een standaardbehandeling met ticagrelor of prasugrel. In de analyse werden twee cohorten van elk 1000 patiënten, gebaseerd op de POPular Genetics studie, met elkaar vergeleken. Patiënten doorliepen eerst een 1-jaars beslisboom gebaseerd op de studie en kwamen daarna in een levenslang Markov model om kosten en effecten te simuleren. In de basisanalyse leidde de genotype-gestuurde strategie tot een toename in quality-adjusted life-years (QALYs) van 8.98 en een afname in de kosten van $€ 725,550.69$ euro per 1000 patiënten. Ook in verschillende scenario analyses, onder andere een waarin de medicijn kosten gelijk waren om de generieke beschikbaarheid van ticagrelor en prasugrel te simuleren, bleef de genotype-gestuurde strategie kosteneffectief.

\section{Heropladen met clopidogrel na het switchen van P2Y12 remmers}

In hoofdstuk 8 werd gekeken of het gebruik van een nieuwe oplaaddosis van de clopidogrel, zoals wordt aangeraden in de huidige richtlijnen, effect heeft op de uitkomsten in de week na het myocardinfarct. Er waren 516 patiënten die geen oplaaddosis kregen en 172 patiënten die wel een oplaaddosis van $300 \mathrm{mg}$ of, in de meeste gevallen, 600mg kregen. In de groep die wel een oplaaddosis kreeg, ontwikkelde niemand een bloeding of een ischemische uitkomst, terwijl in de groep zonder oplaaddosis vier patiënten een ischemische uitkomst en drie patiënten een bloeding hadden. Doordat dit slechts kleine groepen zijn en de groepen niet gerandomiseerd waren kunnen er geen harde conclusies aan worden verbonden, maar het geven van een nieuwe oplaaddosis lijkt veilig. 


\section{Impact section}

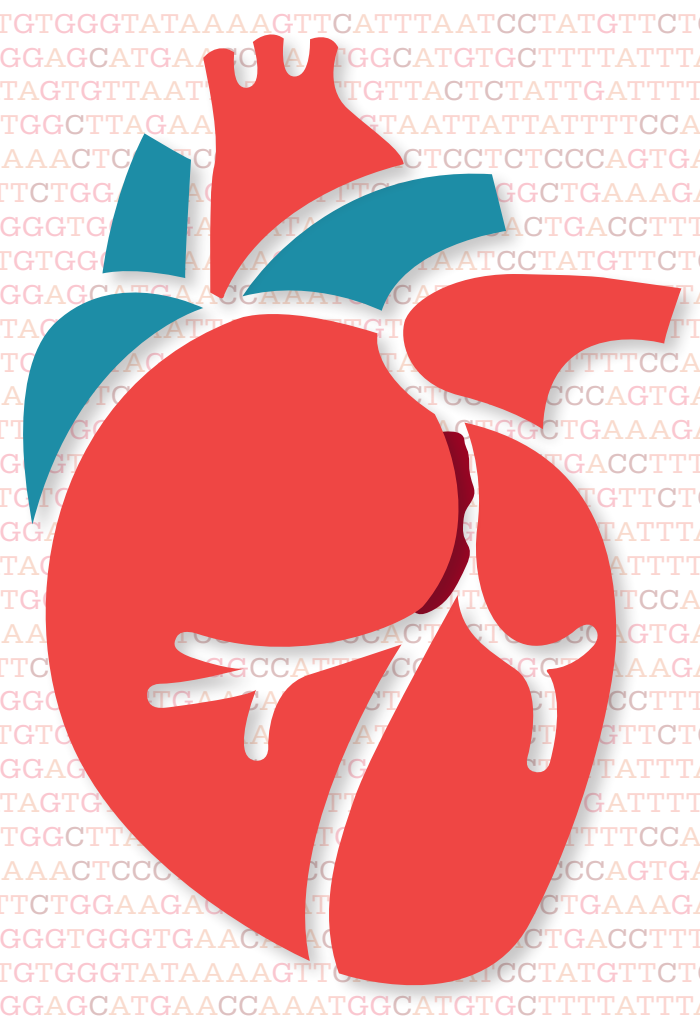




\section{Relevance}

Cardiovascular diseases remain the leading cause of death worldwide and can significantly reduce quality of life and economic output. In the Netherlands, more than 34 thousand people are diagnosed with myocardial infarction each year. It has been well established that in these patients, antiplatelet treatment with the $\mathrm{P}_{2} \mathrm{Y}_{12}$ inhibitors ticagrelor, prasugrel or clopidogrel helps to prevent life threatening events like myocardial infarction, stent thrombosis and stroke. Unfortunately, this comes at the cost of increased bleeding complication rates. These occur in up to $30 \%$ (depending on the selected population) of the patients in the year after myocardial infarction and require medical attention. Therefore, it is important to reduce the amount of bleeding complications in order to improve quality of life, reduce healthcare costs and reduce overall mortality. Clopidogrel does not reach its optimal effect in approximately $30 \%$ of the population, which is mostly caused by mutations in the CYP2C19 gene that encodes the eponymous enzyme. Patients treated with clopidogrel and carrying these loss-of-function mutations experience more atherothrombotic events, while the other $\mathrm{P}_{2} \mathrm{Y}_{12}$ inhibitors lead to more bleeding complications. Therefore, this thesis aimed to investigate whether using $C Y P 2 C 19$ genetic testing to guide antiplatelet therapy could improve patient outcomes by reducing bleeding complication rates without increasing atherothrombotic event rates like myocardial infarction, stroke and death.

\section{Target groups}

The results of this thesis are of interest to clinicians who are directly involved in the treatment of patients who have had an acute myocardial infarction. Primarily, this is the case with cardiologists who are responsible for implementing the strategy in clinical practice, but also for primary care physicians and specialists like surgeons who might have to treat bleeding complications that occur during treatment with $\mathrm{P}_{2} \mathrm{Y}_{12}$ inhibitors. Furthermore, clopidogrel is used frequently by neurologists as well and CYP2C19 genetic testing is of increasing interest in patients with stroke, especially in patients with recurrent strokes while being treated with clopidogrel. Results of this thesis could help to start new prospective trials to investigate whether $C Y P 2 C 19$ genetic testing could improve outcomes in stroke patients as well. This thesis is also of interest to health insurance companies and policy makers since a genotype-guided strategy could help to reduce healthcare costs while improving patient outcomes and quality of life.

\section{Implication and implementation}

A CYP2C19 genotype-guided strategy resulted in fewer bleedings without an increase in events like myocardial infarction, stroke and death. These findings were published in the New England Journal of Medicine, which is one of the most impactful medical journals worldwide. It already has been cited in dozens of publications in the short period since its publication and has led to the genotype-guided strategy receiving a recommendation in the latest European Society of Cardiology guideline. This demonstrates the major impact of the trial and the interest for this topic of cardiologists and researchers worldwide. Many are interested in, or have already implemented the strategy in clinical practice. However, it is crucial that health insurance companies or governments reimburse the costs of the genetic test. The health economic analysis of the POPular Genetics demonstrated that a genotype-guided strategy results in improved quality of life, while reducing healthcare costs. Therefore, since in the Netherlands some hospitals have already implemented the strategy, insurance companies and governments are currently the ones saving costs, while hospitals face higher costs with buying the genetic tests. To ensure a broad implementation of the strategy in clinical practice, health insurance companies should cover these costs. 


\section{Curriculum vitae}

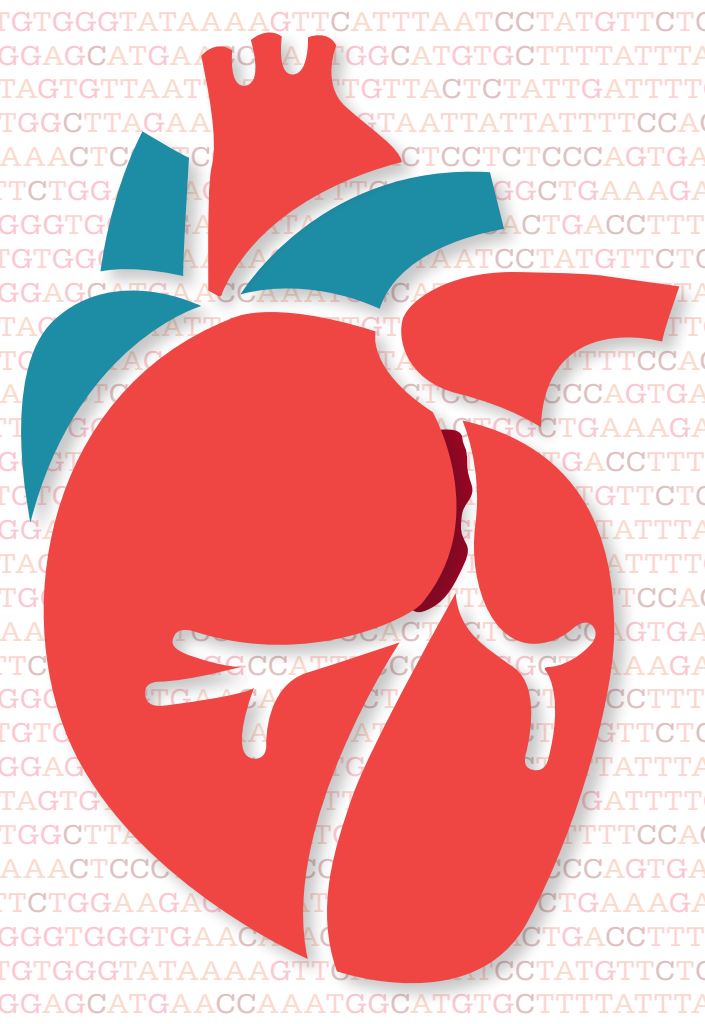




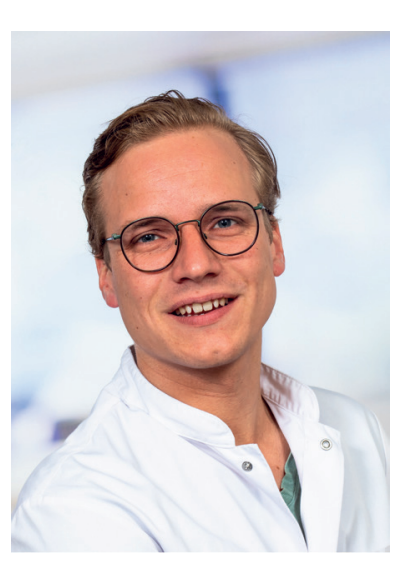

Daniel Marcus Franciscus Claassens was born on March $14^{\text {th }} 1993$ in Rotterdam, the Netherlands. He attended the Grotius College in Delft between 2004 and 2010. In 2010 he obtained his Gymnasium diploma and in addition his International Baccalaureate $\mathrm{A} 2$ in English. After graduation, he studied medicine at Utrecht University. He obtained his bachelor degree in 2013 and his master degree in 2017. He quickly gained interest in the field of cardiology and started doing research at the St. Antonius Hospital in Nieuwegein while still in university under supervision of prof. dr. J.M. ten Berg. After graduation as a medical doctor, he became a fulltime $\mathrm{PhD}$ candidate at the department of cardiology in the St. Antonius Hospital. He was responsible for coordinating the international multicenter randomized POPular Genetics trial and worked under supervision of prof. dr. J.M. ten Berg, prof. dr. A.W.J. van 't Hof, dr. V.H.M. Deneer and dr. R.S. Hermanides. He held presentations at several national and international congresses, including a hotline session at the European Society of Cardiology congress in Paris in 2019. He won a price for best presentation at the St. Antonius wetenschapsavond in 2019 and he reached $2^{\text {nd }}$ place for best research in the field of cardiovascular medicine in the Netherlands from the Werkgroep Cardiologische centra Nederland (WCN) 2019-2020.

In January 2020, he started working as a resident (ANIOS) at the department of cardiology in the St. Antonius Hospital and in December 2020, he started working as a resident (ANIOS) at the department of cardiology in the Isala. He will start his residency in cardiology (AIOS) at the department of internal medicine in the Isala in December 2021.

\section{List of publications}

Al-Rubaish, AM, Al-Muhanna FA, Alshehri AM, Al-Mansori MA, Alali RA, Khalil RM, Al-Faraidy KA, Cyrus C, Sulieman MM, Vatte C, Loza BL, Claassens DMF, Asselbergs FW, Al-Ali A. Bedside testing of CYP2C19 vs. conventional clopidogrel treatment to guide antiplatelet therapy in ST-segment elevation myocardial infarction patients. Int J Cardiol 2021; doi: 10.1016/j.ijcard.2021.08.051

Claassens DMF, Van Dorst WM, Vos GJA, Bergmeijer TO, Hermanides RS, Van 't Hof AWJ, Van der Harst P, Barbato E, Morisco C, Tjon Joe Gin RM, Asselbergs FW, Mosterd A, Herrman JPR, Dewilde WJM, Postma MJ, Deneer VHM, Ten Berg JM, Boersma C. Cost-effectiveness of a CYP2C19 genotype-guided strategy in acute myocardial infarction patients: Results from the POPular Genetics trial. Am J Cardiovasc Drugs 2021; doi: 10.1007/s40256-021-00496-4

Mahmoodi BK, Eriksson N, Ross S, Claassens DMF, Asselbergs FW, Meijer K, Siegbahn A, James S, Paré G, Wallentin L, Ten Berg JM. Factor V Leiden and the risk of bleeding in patients with acute coronary syndromes treated with antiplatelet therapy: pooled analysis of three randomized clinical trials. J Am Heart Assoc 2021; 10:e21115

Al-Rubaish AM, Al-Muhanna FA, Alshehri AM, Alsulaiman AA, Alabdulali MM, Alkhamis F, Alamri AS, Alali RA, Akhtar MS, Cyrus C, Claassens DMF, Asselbergs FW, Al-Ali AK. Prevalence of CYP 2 C19*2 carriers in Saudi ischemic stroke patients and the suitability of using genotyping to guide antiplatelet therapy in a university hospital setup. Drug Metab Pers Ther 2021; doi: 10.1515/dmdi-2021-0104

Tavenier AH, Claassens DMF, Hermanides RS, Vos GJA, Bergmeijer TO, Kelder JC, Deneer VHM, van 't Hof AWJ, Ten Berg JM. Efficacy and safety of glycoprotein IIb/IIIa inhibitors in addition to P2 $\mathrm{Y}_{12}$ inhibitors in ST-segment elevation myocardial infarction: A subanalysis of the POPular Genetics trial. Catheter Cardiovasc Interv 2021; doi: 10.1002/ccd.29861

Claassens DMF, Gimbel ME, Bergmeijer TO, Vos GJA, Hermanides RS, Van der Harst P, Barbato E, Morisco C, Tjon Joe Gin RM, De Very E, Heestermans TACM, Jukema JW, Von Birgelen C, Waalewijn RA, Hofma 
SH, Den Hartog FR, Voskuil M, Van 't Hof AJW, Asselbergs FW, Mosterd A, Herrman JPR, DeWilde W, Mahmoodi BK, Deneer VHM, Ten Berg JM. Clopidogrel in noncarriers of CYP2C19 loss-of-function alleles versus ticagrelor in elderly patients with acute coronary syndrome: A prespecified sub analysis from the POPular Genetics and POPular Age trials. Int J Cardiol 2021; 334:10-17

Claassens DMF, Bergmeijer TO, Vos GJA, Hermanides RS, Van 't Hof AJW, Van der Harst P, Barbato E, Morisco C, Tjon Joe Gin RM, Asselbergs FW, Mosterd A, Herrman JPR, Dewilde WJM, Janssen PWA, Kelder JC, Mahmoodi BK, Deneer VHM, Ten Berg JM. Clopidogrel versus ticagrelor or prasugrel after primary percutaneous coronary intervention according to CYP2C19 genotype: A POPular Genetics subanalysis. Circ Cardiovasc Interv 2021; 14:e009434

Chan Pin Yin DRPP, Vos GA, van der Sangen NMR, Walhout R, Tjon Joe Gin RM, Nicastia DM, Langerveld J, Claassens DMF, Gimbel ME, Azzahhafi J, Bor WL, Oirbans T, Dekker J, Vlachojannis GJ, van Bommel RJ, Appelman Y, Henriques JPS, Kikkert WJ, ten Berg JM. Rationale and design of the Future Optimal Research and Care Evaluation in Patients with Acute Coronary Syndrome (FORCE-ACS) Registry: Towards "Personalized Medicine" in daily clinical practice. J Clin Med 2020; 9:E3173

Claassens DMF, Sibbing D. De-escalation of antiplatelet treatment in patients with myocardial infarction who underwent percutaneous coronary intervention: A review of the current literature. J Clin Med 2020; 9:E2983

Al-Rubaish AM, Al-Muhanna FA, Alshehri AM, Al-Mansori MA, Alali RA, Khalil RM, Al Faraidy KA, Cyrus C, Sulieman MM, Vatte C, Claassens DMF, ten Berg JM, Asselbergs FW, Al-Ali AK. Bedside testing of CYP2C19 gene for treatment of patients with PCI with antiplatelet therapy. BMC Cardiovasc Disord. 2020; 20:268

Claassens DMF, Tavenier AH, Hermanides RS, Vos GJA, Hinrichs DL, Bergmeijer TO, van 't Hof AWJ, Deneer VHM, ten Berg JM. Reloading when switching from ticagrelor to prasugrel within 7 days after STEMI. JACC cardiovascular interv. 2020; 13:663-665

Claassens DMF, Ten Berg JM. Genotype-guided treatment of oral P2Y12 inhibitors: where do we stand? Pharmacogenomics 2020; 21:83-86
Claassens DMF, Vos GJA, Bergmeijer TO, Hermanides RS, van 't Hof AWJ, van der Harst P, Barbato E, Morisco C, Tjon Joe Gin RM, Asselbergs FW, Mosterd A, Herrman JR, Dewilde WJM, Janssen PWA, Kelder JC, Postma MJ, de Boer A, Boersma C, Deneer VHM, ten Berg JM. A Genotype-Guided Strategy for Oral P2Y12 Inhibitors in Primary PCI. N Engl J Med 2019; 381:1621-1631

Ten Berg JM, Claassens DMF. Will intravenous paracetamol crush fentanyl. Neth Heart J. 2019; 27:169-170

Claassens DMF, Coers DO, Bergmeijer TO, Chan Pin Yin DRPP, Ten Berg JM. Is there a place for heart-type fatty acid binding protein in the era of high sensitive cardiac troponin $\mathrm{T}$ for the diagnosis of acute myocardial infarction? A systematic review. Emergency Care Journal 2019;15: https://doi.org/10.4081/ecj.2019.8049

Bergmeijer TO, Vos GJ, Claassens DMF, Janssen PW, Harms R, der Heide RV, Asselbergs FW, ten Berg JM, Deneer VH. Feasibility and implementation of CYP2C19 genotyping in patients using antiplatelet therapy. Pharmacogenomics 2018; 19:621-628

Janssen PWA, Claassens DMF, Willemsen LM, Bergmeijer TO, Klein P, ten Berg JM. Perioperative management of antiplatelet treatment in patients undergoing isolated coronary artery bypass grafting in Dutch cardiothoracic centres. Neth Heart J. 2017; 25:482-489 


\section{Dankwoord}

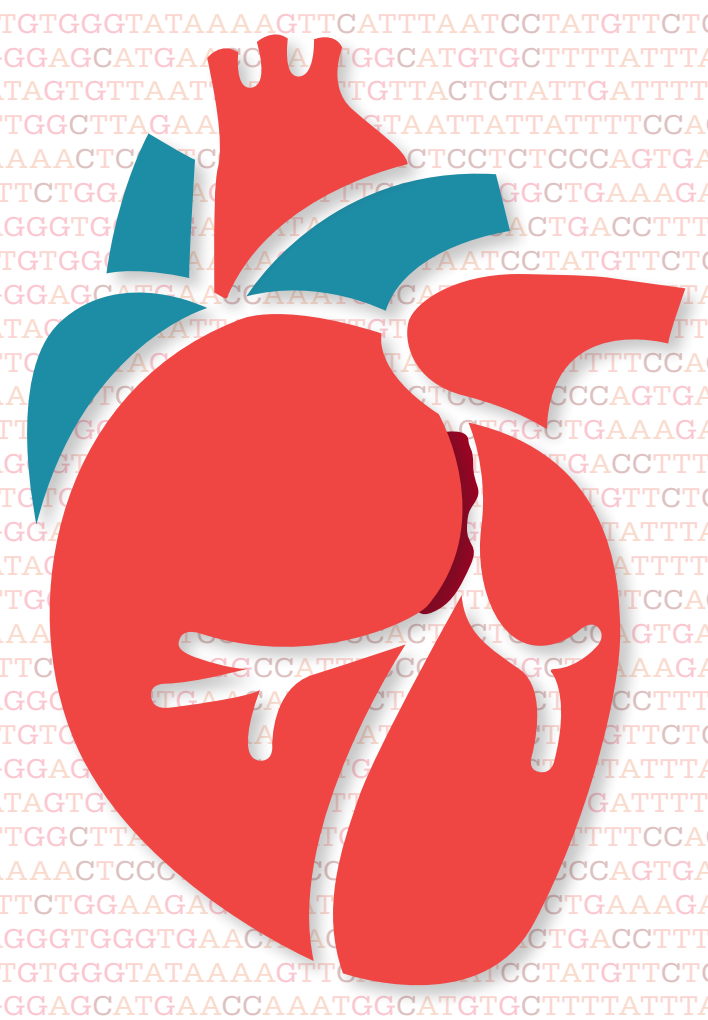


Een proefschrift komt altijd tot stand met de hulp van velen en hier wil ik er een aantal in het bijzonder bedanken. Als eerste alle patiënten die hebben deelgenomen aan de POPular Genetics studie. Een hartinfarct is een heftig moment om mee te maken, maar gelukkig waren jullie bereid om zo kort na opname mee te werken aan het onderzoek. Zonder jullie was dit werk nooit tot stand gekomen.

Mijn promotoren, professor ten Berg en professor van 't Hof.

Beste Jur. Ik ben blij dat je mij hebt aangenomen als arts-onderzoeker en ben je dankbaar dat ik de kans heb gekregen om zo'n grote studie te coördineren waaruit uiteindelijk een aantal fantastische publicaties zijn gekomen. Ik heb in de afgelopen jaren veel van jou geleerd, maar ook vee zelf mogen uitzoeken. Deze ervaring neem ik de rest van mijn leven mee.

Beste Arnoud, ik wil je bedanken voor je hulp bij de POPular Genetics studie en de commentaren die jij bij alle stukken hebt gegeven.

Mijn copromotoren dr. Deneer en dr. Hermanides.

Beste Vera, zonder jou was de POPular Genetics nooit tot stand gekomen. Toen ik als onderzoeker begon was jij helaas net vertrokken naar het UMC Utrecht, maar ook vanuit daar ben je betrokken gebleven en heb je kritisch meegekeken naar alle publicaties en presentaties. Bedankt daarvoor.

Beste Rik, zonder jouw inzet voor de studie in het Isala was de inclusie periode nog veel langer geweest. Ik ben je niet alleen dankbaar voor je hulp bij de studie, maar ook voor je ondersteuning nu ik ook in het Isala werk. Ik hoop komende jaren nog veel van jou te mogen leren.

De leden van de beoordelingscommissie, Prof.dr. Hackeng, Prof.dr. Ten Cate, Prof.dr. Geuns, Prof.dr. Henriques en Prof.dr. Vernooy, wil ik bedanken dat zij plaats wilde nemen in de commissie.

Ik wil alle hoofdonderzoekers van de POPular Genetics studie, professor Van der Harst, professor Asselbergs, professor Barbato, professor Morisco, dr. Mosterd, drs. Tjon Joe Gin, dr. Dewilde en ook al het overige personeel uit alle deelnemende centra (Isala Zwolle, UMC Groningen, Rijnstate Arnhem, Meander MC Amersfoort, UMC Utrecht, Amphia Breda, OLVG Amsterdam, OLVZ Aalst en University Federico II, Napels), zoals arts assistenten, onderzoekers en R\&D personeel, zonder wie de studie niet geslaagd zou zijn, bedanken.

Aan alle medewerkers van het farmatox lab, Kees, Lilian, Niki, Peter, Remko, Richard, Sylvia, Tamimount, die al die jaren de duizenden buisjes bloed verwerkt hebben en de genotypering testen hebben uitgevoerd: bedankt daarvoor.

Lars van Raaij, bedankt voor al jouw ondersteuning met het verwerken en verzenden van de vragenlijsten, het invoeren van data en andere klussen die jij voor de studie gedaan hebt.

Hans Kelder, bedankt voor al je hulp met, en adviezen over statistiek en epidemiologie. Zonder jouw hulp zou het nooit gelukt zijn om alle statistiek op tijd af te krijgen voor de New England en de ESC-deadlines en je stond altijd klaar voor vragen die ik of wetenschapsstudenten hadden.

Paul, wij ontmoetten elkaar in het Antonius alweer bijna 10 jaar geleden, toen ik nog als werkstudent bij de R\&D kwam. Daarna ben ik via jou als werkstudent doorgegaan bij o.a. de POPular Genetics en hebben we samen onderzoek gedaan toen ik nog studeerde, wat uiteindelijk tot mijn eerste publicatie heeft geleid. Bedankt voor jouw hulp en begeleiding in he begin, jouw bijdrage aan de studie en het contact dat we nog steeds hebben en hopelijk ook blijven houden.

Thomas, jij bent de drijvende kracht geweest achter het opzetten van de POPular Genetics studie en je bent daar altijd bij betrokken gebleven. Of het nu was met patiënten includeren en data verzamelen in Amersfoort, het geven van tips over het uitvoeren van onderzoek of kritisch kijken naar de verschillende manuscripten. Bedankt voor al je hulp en inzet.

Gert Jan, ook zonder jouw bijdrage zou de studie niet zijn afgerond. Zonder jouw kennis van ' $R$ ' zou het genereren en verwerken van de vragenlijsten een nog veel grotere klus zijn geweest dan dat het nu al was. Ook was he zonder jou niet mogelijk geweest om de data op een makkelijke manier te exporteren. Wij hebben een aantal hele mooie publicaties samen en ik wens je veel succes met het afronden van je promotie.

Jorn, al in ons laatste jaar van de studie hebben we veel samen opgetrokken en dat ging ook door toen we allebei ongeveer tegelijkertijd startten als onderzoekers. We kwamen veel dezelfde zaken tegen bij onze studies waardoor we elkaar konden helpen, en ook buiten werk hebben we veel leuke dingen ondernomen. Helaas werken we nu niet meer samen, maar ik wens je veel succes met het afronden van je promotie en de opleiding tot cardioloog. Hopelijk zijn we in de toekomst ook weer collega's en kunnen we weer samen naar congressen en borrels.

Dean, we startten op hetzelfde moment als onderzoeker en ik heb het altijd erg gezellig met jou gehad. De FORCE ACS welke jij overgenomen hebt zal denk ik nog tot enkele mooie publicaties lijden. Je kon altijd helpen met 
inclusies als ik weer eens ergens in het land op reis was en op borrels kon ik altijd enorm om je lachen. Veel succes met het afronden van jouw promotie traject en hopelijk zien we elkaar in de toekomst nog vaker.

Marieke, samen hebben we ruim 2,5 jaar lang een hok gedeeld. Gelukkig kon je mijn muziek altijd waarderen. Bedankt voor het beantwoorden van al mijn vragen en je hulp bij de studie als ik weer eens op pad was. Succes met de laatste loodjes voor jouw eigen promotie.

Alle andere onderzoekers waar ik korter of langer mee heb samengewerkt, Anne, Bashak, Friso, Harold, Joyce, Kai, Laura, Lisette, Marloes, Martijn, Mitch, Moniek en Wilbert: bedankt voor de gezellige werkdagen, het beantwoorden van vragen en de leuke feestjes, congressen en activiteiten die we samen meegemaakt hebben. Ik hoop jullie in de toekomst nog vaak tegen te komen op de werkvloer of op congressen.

Alle studenten die korter of langer voor mij gewerkt hebben: Dario, Elske, Evelien, Frank, Joëlle, Lucas, Mirte, Sanne, Sjoerd, Stef, Stephanie, Vivianne, Wiesje, en wellicht nog een paar die ik ben vergeten, bedankt voor jullie hulp met het invoeren van alle data en andere ondersteunende werkzaamheden. Daarnaast wil ik natuurlijk ook de vele studenten die hebben meegeholpen aan de POPular Genetics studie voordat ik als artsonderzoeker begon bedanken.

Alle medewerkers van de R\&D-cardiologie die klaar stonden voor ondersteuning. In het bijzonder Boudewijn Uppelschoten, die altijd een voorraad kantoorbenodigdheden had liggen en tevens alle studie monitoren ontving. En ook Mike Bosschaert die klaar stond met ICT-hulp bij bijvoorbeeld REDCap en het randomisatie programma van de POPular Genetics studie.

Petra, Mady en Mary wil ik bedanken voor hun ondersteuning bij het regelen van contracten, handtekeningen, accounts, vergaderingen betalingen en andere administratieve zaken die bij onderzoek doen horen.

Alle arts-assistenten uit het St. Antonius ziekenhuis in Nieuwegein en het Isala in Zwolle, bedankt voor jullie hulp, lessen in de kliniek en de leuke tijd op de werkvloer en bij andere evenementen. Helaas is dat door COVID behoorlijk beperkt het afgelopen jaar. Hopelijk komt daar snel weer verandering in.

Alle cardiologen uit het St. Antonius ziekenhuis en het Isala, bedankt voor alles wat jullie mij in de afgelopen 2 jaar al geleerd hebben. Ik hoop in de komende jaren nog veel van jullie op te steken.
Mijn clubgenoten, Alex, Bruce, Erik, Guy, Joost, Koen, Roy, Stijn en Wouter, bedankt voor de ontelbare gezellige avonden, en leuke feestjes, festivals en weekenden die voor de nodige afleiding zorgen. We hebben er nu ruim 10 jaar opzitten en ik hoop dat we dit nog vele tientallen jaren blijven doen, want om hard te kunnen werken is het af en toe ook nodig om stoom af te blazen.

Mijn oud-huisgenoten, Jolanthe, Kelly en Ralf, wat heb ik van de jaren dat wij samen hebben gewoond genoten en wat kan ik nog steeds genieten van onze reünie avonden. Door de gin-tonic escaleert het na al die jaren toch elke keer weer.

Mijn ouders en broertje, Rob, Marion en Jeroen, jullie hebben het al ruim 28 jaar met mij te stellen. Bedankt voor alle leuke momenten, goede adviezen en ondersteuning die jullie in al deze jaren geboden hebben. Ondanks dat ik het wellicht niet altijd laat merken, ben ik hartstikke blij met jullie.

Mijn liefste Marieke, in al die jaren dat we inmiddels samen zijn hebben wij al heel wat voor- en tegenspoed meegemaakt. Zowel met betrekking tot mijn onderzoek, waar je altijd bereid was om stukken te lezen en te verbeteren, als daarbuiten, sta je altijd voor mij klaar. Ook heb je heel vee tijd geïnvesteerd om dit boekje er netjes uit te laten zien. Dankjewel hiervoor en ik hoop dat wij nog vele jaren samen gelukkig zullen zijn. 
